Universidad deValladolid

PROGRAMA DE DOCTORADO EN INVESTIGACIÓN EN CIENCIAS DE LA SALUD

TESIS DOCTORAL:

\title{
REHABILITACIÓN INTEGRAL POST-ICTUS: FACTORES SOCIALES, IMPACTO A LARGO PLAZO Y TRABAJO SOCIAL DE NEUROLOGIA
}

Presentada por Verónica Olmedo Vega para optar al grado de Doctora por la Universidad de Valladolid

Dirigida por:

María José Aguilar Idáñez

Juan Francisco Arenillas Lara 
A mis hijos. Mis padres y hermana.

Especialmente a ti Sergio. 


\section{ÍNDICE}

Agradecimientos $\quad 5$

Difusión científica $\quad 6$

Resumen $\quad 7$

Abstract 9

Abreviaturas 11

Índice de gráficos $\quad 12$

$\begin{array}{ll}\text { Índice de tablas } & 13\end{array}$

Capítulo 1. INTRODUCCIÓN 14

1. EL ICTUS EN EL CONTEXTO DE LAS ENFERMEDADES NEUROLÓGICAS CON MAYOR IMPACTO SOCIOSANITARIO

1.1. Concepto de ictus 15

1.2. Impacto personal, familiar y sociosanitario del ictus 16

1.2.1.Impacto en la persona 17

$\begin{array}{ll}\text { 1.2.2.Impacto familiar } & 18\end{array}$

$\begin{array}{ll}\text { 1.2.3.Impacto sociosanitario } & 19\end{array}$

2. LA REHABILITACIÓN INTEGRAL. CONCEPTO. DIMENSIÓN HOLÍSTICA E INTEGRAL DE LA PERSONA

2.1. ¿Qué es la rehabilitación integral? 21

$\begin{array}{ll}\text { 2.1.1. Modelo holístico } & 22\end{array}$

2.2. El proceso de rehabilitación integral del ictus 23

2.2.1. Componente funcional 24

2.2.2. Componente profesional 30

$\begin{array}{ll}\text { 2.2.3. Componente social } & 31\end{array}$

2.3. Finalidad y beneficios de la rehabilitación integral post-ictus 33

3. IMPORTANCIA DE LOS FACTORES PERSONALES Y SOCIOFAMILIARES EN EL ACCESO A REHABILITACIÓN INTEGRAL POST-ICTUS Y EN SU PRONÓSTICO DE RECUPERACIÓN

3.1. Factores personales 36

3.1.1. Sexo y edad 36

3.1.2. Estado civil o tener pareja 38

3.1.3. Unidad de convivencia 40

3.1.4. Lugar de residencia 41

3.1.5. Vivienda $\quad 42$

3.1.6. Economía 44

3.1. Factores sociofamiliares 45

3.2.1. Apoyo familiar $\quad 45$

3.2.2. Apoyo social 46 
4. PAPEL DEL TRABAJO SOCIAL NEUROLÓGICO EN EL HCUV EN EL ACCESO A LA RHB INTEGRAL POST-ICTUS Y RECUPERACIÓN DEL PACIENTE

4.1. Origen

4.2. Perfil del Trabajo Social Neurológico (TSN) en el HCUV

4.3. Proceso de intervención de Trabajo Social Neurológico en la rehabilitación integral post-ictus

4.3.1. Acogida

4.3.2. Anamnesis social

51

4.3.3. Valoración multidisciplinar

54

4.3.5. Planificación del tratamiento rehabilitador al alta hospitalaria

58

4.3.6. Alta hospitalaria

65

4.3.7. Seguimiento post-alta hospitalaria

65

4.3.8. Reinserción socio-profesional del paciente

1. ÁMBITO DE ESTUDIO Y DISEÑO METODOLÓGICO

2. SELECCIÓN DE PACIENTES/POBLACIÓN DE ESTUDIO 74

2.1. Criterios de inclusión y exclusión $\quad 75$

2.2. Tamaño muestral

3. PROTOCOLO DE RECOGIDA DE DATOS Y SEGUIMIENTO 76

3.1. Base de datos 76

3.2. Recogida de datos 76

3.3. Variables de estudio 77

3.4. Descripción del proceso: Temporalidad 81

4. INSTRUMENTOS DE MEDIDA/ESCALAS UTILIZADAS 83

4.2. Nihss $\quad 83$

4.2. Escala Modificada de Rankin (mRs) 83

4.3. Escala Gijón 84

4.4. Apgar familiar $\quad 84$

4.5. Índice Barthel $\quad 85$

5. ANÁLISIS ESTADÍSTICO 85

Capítulo 4. RESULTADOS $\quad 87$

1. ANÁLISIS DESCRIPTIVO DE LA MUESTRA DE ESTUDIO 88

1.1. Primer estudio: Análisis de los factores asociados a la recuperación integral de pacientes de ictus al alta de Hospital de Agudos 
1.2. Segundo estudio: Estudio del impacto de la RHB integral sobre la recuperación funcional a largo plazo de los pacientes con ictus

2. RESULTADOS VARIABLES ASOCIADAS A REHABILITACIÓN INTEGRAL

2.1. Primer estudio: Análisis de los factores asociados a la recuperación integral de pacientes de ictus al alta de Hospital de Agudos

2.2. Segundo estudio: Estudio del impacto de la RHB integral sobre la recuperación funcional a largo plazo de los pacientes con ictus

3. IMPACTO DE LA RHB INTEGRAL POST-ICTUS SOBRE EL PRONÓSTICO

FUNCIONAL Y SITUACIÓN A LARGO PLAZO DE LOS PACIENTES ESTUDIADOS (5 AÑOS)

3.1. Acceso a RHB integral post-ictus $\quad 99$

3.2. Evolución funcional 100

Capítulo 5. DISCUSIÓN 103

1. ACCESO DE LOS PACIENTES A REHABILITACIÓN INTEGRAL POST-ICTUS 104

2. FACTORES ASOCIADOS AL ACCESO A REHABILITACIÓN INTEGRAL POST-ICTUS 104

3. EFECTOS DE LA REHABILITACIÓN INTEGRAL POST-ICTUS A LARGO PLAZO 106

4. LABOR DEL TRABAJO SOCIAL NEUROLÓGICO EN EL ACCESO Y CUMPLIMIENTO DEL TRATAMIENTO REHABILITADOR INTEGRAL POST-ICTUS 107

$\begin{array}{ll}\text { 4.1. Visión integral } & 107\end{array}$

4.2. Barreras sociales de acceso y mantenimiento del tratamiento rehabilitador integral post-ictus

4.2. Manejo de los recursos al alta hospitalaria 108

4.3. Motivación-empoderamiento del paciente-familiar 108

4.4. Seguimiento social continuo 108

5. LIMITACIONES Y PROSPECTIVA 109

Capítulo 6. CONCLUSIONES 112

$\begin{array}{ll}\text { BIBLIOGRAFÍA } & 114\end{array}$

$\begin{array}{ll}\text { ANEXOS } & 131\end{array}$

Anexo 1: PUBLICACIONES DERIVADAS DE LA TESIS 132

Anexo 2: ESCALAS DE REGISTRO 179

Anexo 3: MAPAS 184 
Agradezco muy especialmente a mis directores de tesis, María José Aguilar Idáñez y Juan Francisco Arenillas Lara. A la Doctora María José Aguilar Idáñez por su gran capacidad de conocimiento y comprensión, sus valores y compromiso con el avance científicotécnico de la profesión de Trabajo Social, aunando los principios de la profesión con la ciencia. Al Doctor Arenillas Lara por su mirada a la persona, antes que a la enfermedad, su capacidad de entendimiento de la dimensión bio-psico-social, su solidaridad y sensibilidad ante las necesidades de las personas que atendemos en el servicio de neurología del Hospital Clínico Universitario de Valladolid.

A ambos por poner a mi disposición su existir emocional, apoyándome y facilitándome todo lo necesario para que pudiese recorrer los caminos de los estudios de doctorado.

A la Universidad de Valladolid, por acogerme como estudiante de Doctorado y a los profesores que tanto conocimiento me han ofrecido durante estos años.

Otro agradecimiento muy especial a M $\mathrm{M}^{\mathrm{a}} \mathrm{Fe}$ Muñoz Moreno, responsable de Metodología y Bioestadística del Hospital Clínico Universitario de Valladolid por su gran apoyo y agilidad en los análisis estadísticos. También agradezco a Susana del Villar Barba, Bibliotecaria-Documentalista del Hospital Clinico Universitario de Valladolid, por hacerme accesible, con celeridad y eficiencia, la bibliografía utilizada para la realización de esta tesis.

Agradezco a mi compañero de viaje, Sergio, por transmitirme la energía y la fortaleza para que culminara los estudios doctorales. Por entender que, mediante el proceso de elaboración de esta tesis, fue necesario realizar sacrificios como momentos a su lado. Gracias a mis hijos por ser la fuente de mi esfuerzo y todas las energías requeridas en esta tesis.

Especialmente a ti, Ma José Aguilar, por tu conocimiento, tiempo y dedicación, sin tu acompañamiento no hubiese logrado recorrer este camino.

A pacientes y familiares que además de sufrir el duro golpe de un ictus se encuentran con dificultades para conseguir ese tratamiento rehabilitador que tanto necesitan, va por vosotros.

"Todo parece imposible hasta que se hace"

Nelson Mandela. 
La investigación doctoral ha generado las siguientes contribuciones científicas:

\section{Publicaciones en revistas indexadas:}

- Olmedo Vega V, Aguilar Idañez MJ, Arenillas Lara JF. Análisis de factores asociados a la recuperación integral a pacientes de ictus al alta de Hospital de Agudos. Revista Española de Salud Pública [Internet]. 2019 [citado el 22 de marzo de 2021];93:1-13. Recuperado a partir de:

https://www.mscbs.gob.es/biblioPublic/publicaciones/recursos_propios/resp/revista cdrom/VOL93/ORIGINALES/RS93C_201910103.pdf

- Olmedo Vega V, Aguilar Idañez MJ, Arenillas Lara JF. Rehabilitación integral postictus: efectos a largo plazo y factores socioambientales condicionantes del acceso". Aceptada y prevista publicación en el mes de julio. Revista de Neurologia (en prensa).

\section{Ponencias en congresos y conferencias de formación profesional especializada:}

- Conferencia "La intervención psicosocial con personas que sufren ictus desde su ingreso hospitalario". Facultad de Ciencias Sociales. Universidad de Salamanca. Salamanca, 28 de octubre de 2019.

- Ponencia invitada en el curso "Propuestas y modelos de intervención centrados en personas con discapacidades, desde el enfoque de vida independiente". Colegio Oficial de Trabajo Social de Palencia y COCEMFECYL. Ámbito Regional. Online, 23-25 de noviembre 2020.

- Conferencia "El papel del trabajo social neurológico en la calidad de vida del paciente que sufre ictus”. Organiza Asociación Española de Trabajo Social y Salud. Delegación de Castilla y León. 2 horas. 22 de junio 2021.

- Ponencia "Ictus: intervención social para el logro de la autonomía personal y la participación social del paciente”. IV Congreso Internacional de Trabajo Social (CIFETS 2021) y XIII Congreso de Facultades y Escuelas de Trabajo Social "Trabajo Social en la Era Digital: Ética y Cuidados”. Valencia, 14-16 de julio 2021.

Premio Científico de la Revista Documentos de Trabajo Social: en modalidad Intervención social por "Las 4 q del trabajo social neurológico: Qué, quién, porque y para qué”. Verónica Olmedo Vega y Sandra Fernández Díez. Recuperado a partir de: https://www.trabajosocialmalaga.org/wp-content/uploads/2021/05/DTS 63 1.pdf 


\section{Introducción:}

La rehabilitación (RHB) integral post-ictus supone tratar de forma coordinada e integrada todos los déficits funcionales que afectan a la persona que ha sobrevivido a un ictus. Se desconoce en nuestro medio cuál es el impacto a largo plazo de la RHB integral sobre la situación funcional de los pacientes. Asimismo, no está claro si el acceso a la RHB integral está garantizado dentro del Sistema Nacional de Salud, y en qué medida está determinado por factores sociales.

\section{Objetivos:}

1) Conocer la probabilidad que tienen los pacientes con ictus en nuestro medio de acceder a la RHB integral tras ser indicada por un equipo multidisciplinar.

2) Analizar los factores sociales y ambientales condicionantes del acceso a rehabilitación integral post-ictus.

3) Evaluar los efectos a largo plazo de la rehabilitación integral en la funcionalidad del paciente.

\section{Material y Métodos:}

Se han realizado dos estudios consecutivos, en el primero de ellos se incluyeron 102 pacientes con ictus isquémico y hemorrágico ingresados en el Hospital Clínico Universitario de Valladolid durante 2016, que precisaban una rehabilitación integral al alta hospitalaria. Se evaluó el acceso a la RHB integral a los tres meses post-ictus en todos los pacientes. Se analizó la asociación entre las variables basales de tipo personal, social y familiar, entre otras, y el acceso a la RHB integral post-ictus. Finalmente, se realizó un modelo de regresión logística multivariable con las variables que mostraron asociación en el análisis bivariable. En el segundo estudio se incluyeron 171 pacientes consecutivos hospitalizados en 2015 en Servicio de Neurología con primer ictus isquémico, sin dependencia funcional previa, candidatos a rehabilitación integral, que fueron seguidos a largo plazo en la consulta de Trabajo Social Neurológico del hospital. Se analizó de forma análoga a lo realizado en el primer estudio la asociación entre variables basales y el acceso a la RHB integral post-ictus. Finalmente, se realizó un modelo de regresión logística multivariable empleando la puntuación en el Barthel Index en la visita final como variable 
dependiente, mientras que el acceso a RHB integral, junto con el resto de las variables basales seleccionadas, se consideraron variables independientes.

\section{Resultados:}

En ambos trabajos se observó un porcentaje similar de acceso a la RHB integral, en torno al 53\%. Los factores asociados de forma independiente al acceso a la RHB integral fueron, en el primer estudio la edad $(\mathrm{p}<0,001)$, el estado civil $(\mathrm{p}=0,007)$ y la existencia de apoyo familiar $(p=0,01)$. La edad y el lugar de residencia (rural o urbano) fueron los factores explicativos del acceso a la rehabilitación integral (se obtuvieron valores ajustados al resto de variables de $\mathrm{p}<0,001)$. En el segundo estudio, con un seguimiento medio de 54 meses, se observó que el acceso a la RHB integral post-ictus se comportó como un factor predictor de buen pronóstico funcional a largo plazo en los 137 pacientes que sobrevivieron hasta el final del seguimiento (OR: 12,441, (IC 95\% 4,7-32,5), $\mathrm{p}<0,001)$. Resultaron variables predictoras del acceso: residencia en medio urbano (OR: 2,957, (IC 95\% 1,067-8,199), p=0,037), complemento con RHB privada (OR: 2,890, IC 95\% 1,130-7,392), p=0,027), mejor Rankin al alta (OR: 22,437, (IC 95\% 3,247-155,058), $\mathrm{p}=0,014)$.

\section{Conclusiones:}

Solo la mitad de la población objeto de estudio accedió a la RHB integral. Se evidencia que la RHB integral post-ictus está asociada a un mejor pronóstico funcional a largo plazo (5 años), pero el acceso a la misma está condicionado por factores ambientales y sociales tales como el lugar de residencia y la posibilidad de contratar servicios privados, entre otros. El Trabajo Social en neurología desempeña un papel clave en la recuperación funcional de la persona tras un ictus, resolviendo las barreras socio ambientales que impiden su acceso al tratamiento rehabilitador integral.

\section{Palabras Clave:}

Ictus, Rehabilitación Integral, Trabajo Social Sanitario, Neurología, Accesibilidad a los servicios de salud, Factores socioeconómicos, Bienestar Social. 


\section{Introduction:}

Comprehensive post-stroke rehabilitation (RHB) involves treating in a coordinated and integrated manner all functional deficits that affect the person who has survived a stroke. It is unknown in our environment what the long-term impact of integral RHB is on the functional status of patients. Furthermore, it is not clear whether access to comprehensive RHB is guaranteed within the National Health System, and to what extent it is determined by social factors.

\section{Objectives:}

1) To know the probability that stroke patients have in our environment have of accessing comprehensive RHB after being indicated by a multidisciplinary team.

2) Analyze the social and environmental factors conditioning access to comprehensive post-ictus rehabilitation.

3) Evaluate the long-term effects of comprehensive rehabilitation on patient functionality.

\section{Material and Methods:}

Two consecutive studies have been carried out, the first of which included 102 patients with ischemic and hemorrhagic stroke admitted to the University Clinical Hospital of Valladolid during 2016, who needed a comprehensive rehabilitation at hospital discharge. Access to integral RHB at three months post-stroke was evaluated in all patients. The association between baseline variables of personal, social and family type, among others, and access to comprehensive post-stroke RHB was analyzed. Finally, a multivariate logistic regression model was performed with the variables that showed association in the bivariate analysis. The second study included 171 consecutive patients hospitalized in 2015 in the Neurology Service with the first ischemic stroke, without previous functional dependence, candidates for comprehensive rehabilitation, who were followed in the long term in the neurological social work consultation of the hospital. The association between baseline variables and access to comprehensive post-stroke RHB was analyzed in a similar way to that performed in the first study. Finally, a multivariate logistic regression model was performed using the score in the Barthel Index at the final visit as a dependent 
variable, while access to integral RHB, together with the rest of the selected baseline variables, were considered independent variables.

\section{Results:}

In both studies, a similar percentage of access to integral RHB was observed, around $53 \%$. The factors independently associated with access to comprehensive RHB were, in the first study, age $(p<0.001)$, marital status $(p=0.007)$ and the existence of family support $(\mathrm{p}=0.01)$. Age and place of residence (rural or urban) were the explanatory factors for access to comprehensive rehabilitation (values adjusted to the rest of the $\mathrm{p}<0.001$ variables were obtained). In the second study, with an average follow-up of 54 months, it was observed that access to comprehensive post-stroke RHB behaved as a predictor of good long-term functional prognosis in the 137 patients who survived to the end of follow-up (OR: 12.441, (95\% CI 4.7-32.5), $\mathrm{p}<0.001)$. Variables predicting access were: residence in urban environment (OR: 2.957, (95\% CI 1.067-8.199), p=0.037), complement with private RHB (OR: 2.890, 95\% CI 1.130-7.392), p=0.027), better Rankin at discharge (OR: 22.437, (95\% CI 3.247-155.058), $\mathrm{p}=0.014)$.

\section{Conclusions:}

Only half of the study population accessed integral RHB. It is evident that the comprehensive RHB post-stroke is associated with a better long-term functional prognosis ( 5 years), but access to it is conditioned by environmental and social factors such as the place of residence and the possibility of contracting private services, among others. Social Work in neurology plays a key role in the functional recovery of the person after a stroke, resolving the socio-environmental barriers that prevent their access to comprehensive rehabilitative treatment.

\section{Key words:}

Ictus, Integral Rehabilitation, Health Social Work, Neurology, Accessibility to Health Services, Socioeconomic Factors, Social Welfare. 


\section{ABREVIATURAS}

ABVD: Actividades Básicas de la Vida Diaria

ACV: Accidente Cerebro Vascular

AIVD: Actividades Instrumentales de la Vida Diaria

AVD: Actividades de la Vida Diaria

CV: Calidad de Vida

CEADAC: Centro Estatal de Atención al Daño Cerebral

CEAPAT: Centro Estatal de Autonomía Personal y Ayudas Técnicas

DCA: Daño Cerebral Adquirido

DPI: Depresión PostIctal

EA: Enfermedad de Alzheimer

ELA: Esclerosis Lateral Amiotrófica

EM: Esclerosis Múltiple

ECA: Ensayos Clínicos Aleatorizados

HCUV: Hospital Clínico Universitario de Valladolid

IB: Índice Barthel

IMSERSO: Instituto de Mayores y Servicios Sociales

LPAPAD: Ley 39/2006, 16 diciembre, de Promoción de la Autonomía Personal y Atención a las personas en situación de Dependencia.

mRs: Rankin

NIHSS: National Institute of Health Stroke Score

OMS: Organización Mundial de la Salud

RHB: Rehabilitación

RHBI: Rehabilitación Integral

SEN: Sociedad Española de Neurología

TSN: Trabajo Social Neurológico

TSS: Trabajo Social Sanitario 
Gráfico 1. Factores de riesgo cerebrovascular

Gráfico 2. Rankin previo al ictus

Gráfico 3. Rankin al alta hospitalaria

Gráfico 4. Porcentaje tratamiento rehabilitador precisaba

Gráfico 5. Modalidad de rehabilitación que reciben.

Figura 1. Distribución del acceso a RHB integral por edad y estado civil

Gráfico 6. Porcentaje de exitus año 2020

Gráfico 7. Acceso a RHB integral

Gráfico 8. Evolución temporal del pronóstico funcional y su relación con la RHB integral 
Tabla 1. Áreas afectadas por ictus y su tratamiento

Tabla 2. Datos sociales comunes de la familia y de la unidad convivencia.

Tabla 3. Valoración clínica paciente

Tabla 4. Valoración trabajo social neurológico

Tabla 5. Tratamiento rehabilitador que precisa al alta hospitalaria

Tabla 6. Indicadores de valoración de la familia

Tabla 7. Coordinación con otros profesionales

Tabla 8. Criterios inclusión y exclusión ambos estudios

Tabla 9. Variables descriptivas primer estudio $(\mathrm{n}=102)$

Tabla 10. Variables descriptivas segundo estudio $(n=171)$

Tabla 11. NIHSS al ingreso hospitalario

Tabla 12. NIHSS al alta hospitalaria

Tabla 13. Rehabilitación según variables basales $(n=102)$

Tabla 14. Variables explicativas acceso a RHBI.

Tabla 15. Relación entre variables basales y acceso RHBI $(n=171)$

Tabla 16. Modelo de regresión logística multivariable para factores predictores de acceso a RHBI. 


\section{Capítulo 1. INTRODUCIÓN}




\section{EL ICTUS EN EL CONTEXTO DE LAS ENFERMEDADES NEUROLÓGICAS CON MAYOR IMPACTO SOCIOSANITARIO}

Los trastornos neurológicos son "enfermedades del sistema nervioso central y periférico, es decir, del cerebro, la médula espinal, los nervios craneales y periféricos, las raíces nerviosas, el sistema nervioso autónomo, la placa neuromuscular, y los músculos"1. Si bien existen más de 600 enfermedades neurológicas, las más frecuentes y extendidas son: Alzheimer, Parkinson, Ictus, Esclerosis Múltiple, Cefaleas y Migrañas, Corea Huntington y Epilepsia.

Se trata, en todos los casos, de un tipo de enfermedades que provocan graves daños no sólo en la salud de quienes las padecen, sino también en su entorno familiar y social, viéndose afectados diversos ámbitos vitales: laboral, social, médico, económico, entre otros. Considerando la sociedad en su conjunto, estos trastornos agrupan padecimientos con importantes repercusiones sociosanitarias y económicas, debido al evidente costo económico relacionado con el tratamiento farmacológico, terapéutico, clínico y de cuidados $^{2}$. A nivel mundial se estima en mil millones el número de personas afectadas por trastornos neurológicos ${ }^{3}$. La morbimortalidad asociada a los desórdenes neurológicos va en aumento y con ello, la potencial discapacidad secundaria a ellos ${ }^{4}$, discapacidad física (motora), sensorial visual, sensorial auditiva, cognitiva (intelectual), mental y discapacidad múltiple

\subsection{Concepto de Ictus}

El ictus es una enfermedad que ocasiona una gran discapacidad en los supervivientes. A diferencia de otras enfermedades, gran parte de la carga global del ictus se debe a sus consecuencias en términos de años vividos en discapacidad ${ }^{5}$. Se caracteriza por un déficit neurológico a consecuencia de una lesión focal del sistema nervioso central de origen $\operatorname{vascular}^{6}$. El término ictus procede del latín y significa golpe o ataque. Hace referencia a cualquier trastorno de la circulación cerebral, generalmente de comienzo brusco, que puede ser consecuencia de la interrupción de flujo sanguíneo a una parte del cerebro ${ }^{7}$ El ictus consiste en una alteración brusca en una zona del parénquima encefálico ${ }^{8}$.

Existen fundamentalmente dos tipos de ictus según el tipo de lesión que ocurra. La isquemia cerebral o ictus isquémico (trombosis, embolia o apoplejía), cuando el problema es la oclusión o taponamiento de un vaso, con lo que la sangre no puede llegar a determinada zona del cerebro, esa parte queda sin oxígeno y los nutrientes que necesita y 
se lesionan. Si esta situación se mantiene el tiempo suficiente, el tejido se muere y ocurre el infarto cerebral ${ }^{7}$. El segundo tipo es la hemorragia cerebral o ictus hemorrágico (derrame o hematoma cerebral). En este caso se produce una rotura de un vaso dentro del cerebro (hemorragia intracerebral) o en sus envolturas (hemorragia subaracnoidea). Esta sangre no se puede liberar al exterior ya que el cerebro está encerrado en los vasos del cráneo, por eso la sangre presiona el tejido blando, impidiendo que éste se oxigene adecuadamente, provocando también la muerte de los tejidos que están comprimidos?

Ya se trate de ictus hemorrágico o isquémico, es una enfermedad cerebrovascular con impacto social creciente. La tasa global de mortalidad por ictus está disminuyendo en países con bajos y altos ingresos; mientras, el número absoluto de personas que han sufrido un ictus, de supervivientes al ictus, y la discapacidad global por ictus es alta y está aumentando ${ }^{9}$. El ictus es la primera causa de discapacidad en adultos ${ }^{10} \mathrm{y}$ la segunda causa de demencia ${ }^{11}$. Dicha discapacidad suele deberse al amplio y heterogéneo número de secuelas que provoca; desde funcionales, hasta psíquicas e intelectuales e incluso sociales $^{12}$, afectando de forma muy importante la vida cotidiana, al alterar de forma diversa y compleja las funciones cognitivas y funcionales ${ }^{13}$. Las seis áreas que se ven afectadas por el ictus (cognitiva, emocional, comunicación y lenguaje, motora, sensitiva y visual) sufren importantes efectos que interactúan y retro actúan unos con otros, generando déficits complejos que van más allá de cada área de función ${ }^{14}$. Es la quinta causa principal de pérdida de productividad, medida por los años de vida ajustados por discapacidad $^{15}$.

La Sociedad Española de Neurología (2018) ${ }^{16}$ afirma que el ictus supone el 70\% de los ingresos hospitalarios neurológicos que se producen en España y es responsable del 3-6\% del gasto total sanitario. Este problema de carácter socio sanitario supone un coste para el Sistema de Salud, pero también para el paciente y su familia provocándole un gran sufrimiento que puede prevenirse a través del apoyo social desde el comienzo de la enfermedad. Se estima que 1 de cada 4 personas sufrirá un ictus a lo largo de su vida. De esta estimación más de 80 millones de supervivientes globales sufrirán secuelas dónde el foco de atención en estrategias de prevención será imprescindible ${ }^{17}$.

\subsection{Impacto personal, familiar y sociosanitario del Ictus}

La aparición de un ictus en la vida de las personas es un hecho casi siempre generador de crisis debido principalmente a la espontaneidad de su manifestación y la amenaza que supone a la integridad física y psíquica de la persona. Significa un impacto imprevisible, 
con afectación cognitiva y funcional ${ }^{13}$, que provoca importantes consecuencias y secuelas en la vida cotidiana de la persona.

\subsubsection{Impacto en la persona}

Restablecer un nuevo equilibrio supone, para la persona afectada, un cambio fuera de su repertorio habitual de respuestas; ya que se encuentra ante muchas tareas de la vida cotidiana que ya no puede abordar por sus propios medios ${ }^{13}$. La preocupación, el temor, la incertidumbre, la impotencia, la angustia y la impaciencia son una constante que aparece en las personas que le sufren. Muchas personas y, por supuesto, muchos profesionales, se ven superadas por las nuevas circunstancias, siendo sus recursos y mecanismos de respuesta habituales, insuficientes para adaptarse al mundo cambiante. Estos cambios, además, cada vez se producen a mayor velocidad, de manera que el número de personas que se ven afectadas por la situación va aumentando ${ }^{13}$.

El ictus, provoca en las personas problemas de tipo psicosocial: trastornos de ansiedad, conflictos familiares, impotencia con la situación, desconocimiento de cómo actuar. La atención al mismo no puede abordarse al margen de los factores psicosociales ${ }^{18}$, los factores psicosociales actúan en la vida de todos $/ \mathrm{as}^{19}$. Es clave conocer cómo interpreta la persona el acontecimiento que sufre. La persona que sufre ictus tiene en muchos casos la posibilidad de recuperación que hace necesario conocer cómo uno interpreta el episodio acontecido, para reconducirle y motivar al paciente a que actué activamente para lograr la máxima funcionalidad posible.

En los sobrevivientes a un primer ictus frecuentemente hay limitaciones permanentes en una o varias funciones ${ }^{20}$. Cerca del $60 \%$ de las personas durante el primer año posterior a un ACV (fase crónica) poseen una disfunción motora residual en su extremidad superior que puede constituirse en una discapacidad física a largo término ${ }^{21}$. Las mayores consecuencias posteriores al ACV son la disfunción y el déficit motores en extremidades $^{22}$. Al menos el $30 \%$ de los sobrevivientes desarrollan una recuperación incompleta de la deficiencia funcional secundaria al proceso isquémico del ACV, y aproximadamente un $20 \%$ adicional requiere de asistencia en actividades de la vida $\operatorname{diaria}^{23}$.

Los pacientes con ictus sufren una disminución de su capacidad funcional para realizar las actividades básicas e instrumentales de la vida diaria, lo que provoca una disminución en su calidad de vida. 


\subsubsection{Impacto familiar}

Las limitaciones tanto físicas como psíquicas que pueden surgir ante la aparición de un ictus, hace que cobre todavía mayor importancia el papel de la familia en el acceso, mantenimiento y apoyo al tratamiento del paciente ${ }^{24}$.

La familia y/o el/la cuidador/a familiar se consideran apoyos fundamentales en el proceso de atención y rehabilitación del paciente. Hay que tener en cuenta que la familia es, en muchas ocasiones, la fuente principal y única con que cuenta el enfermo para afrontar con éxito los problemas que surgen con la enfermedad. Por ello, es necesario identificar si el paciente tenía previamente a la aparición del ictus un/a cuidador/a familiar, el parentesco de quién ejercía el cuidado de esa persona, si desempeñaba un buen cuidado y qué tareas desempeñaba ${ }^{24}$.

El impacto del ictus en la familia es significativo. Las familias viven muy tristemente que una persona de su entorno sufra una enfermedad. Los familiares de las personas que sufren ictus se deben enfrentar al trauma inicial y los cambios que se producen de forma inesperada con la llegada del ictus a sus vidas. Se ven afectadas las relaciones interpersonales y a menudo las familias tienen una sensación de soledad, dificultades para afrontar y buscar alternativas para una adecuada atención del paciente ${ }^{24}$. El ictus les provoca, en muchos casos, la no aceptación de la realidad, la desestabilización o la unión familiar, pues no deja indiferente a la familia teniendo que asumir nuevos roles y nuevas dinámicas familiares ${ }^{25}$. Por ello, es de vital importancia intervenir también con la familia, al ser una enfermedad impredecible que genera cambios en la vida familiar, disminuyendo el estrés que ha ocasionado esta nueva situación, contribuyendo al reajuste de una nueva adaptación ${ }^{13}$.

Los cambios que empiezan a producirse en sus vidas desde el ingreso hospitalario por un ictus implican un apoyo y acompañamiento social desde el inicio ${ }^{24}$. Estos cambios cobran un rumbo diferente, cuando se prolonga en el tiempo el cuidado intenso del familiar, propiciando la aparición de problemas que son necesarios prevenir. En la familia afloran estados de estrés, ansiedad, alteraciones del comportamiento, conflictos familiares, disminución del rendimiento laboral, absentismo laboral, entre otros ${ }^{24}$. La aparición de estos síntomas inevitablemente repercutirá en el familiar que sufre ictus, y sobre la calidad del cuidado que se le ha de prestar y la rehabilitación del mismo. El cuidado del/la cuidador/a, es fundamental, para mejorar la calidad de vida y recuperación tanto del paciente, como de la persona que le cuida ${ }^{24}$. 


\subsubsection{Impacto sociosanitario}

En el año 2025, 1.200.000 españoles/as habrán sobrevivido a un ictus, pero de no evitarse, más de 500.000 tendrán discapacidad: una cifra considerable ${ }^{26}$ y con entidad suficiente para plantearse y cuestionarse si, como profesionales del Sistema de Salud, podemos hacer algo para evitar que la persona que sufre ictus acabe con una discapacidad.

En 2017 se publicaron, fruto de una exhaustiva investigación, los costes estimados desde el punto de vista social, divididos en asistencia sanitaria directa (hospitalaria, ambulatoria y medicación), asistencia no sanitaria directa (principalmente asistencia formal e informal) y pérdidas de productividad laboral. El coste de los pacientes ingresados en unidades de ictus en España es de 27.711 euros anuales por paciente. Más de dos tercios son costos sociales, principalmente cuidado informa ${ }^{27}$ Los ictus siguen siendo una carga importante para los Sistemas de Salud y para la sociedad, por lo que se necesitan esfuerzos adicionales para su prevención.

Tanto las sociedades científicas como el Sistema Nacional de Salud apoyaron una estrategia para transferir estos avances de manera clara y consistente a la práctica clínica con el objetivo de que todos los/las pacientes con ictus reciban el mismo grado de atención especializada, de acuerdo con la etapa de su enfermedad, independientemente del lugar donde vivan, su edad, sexo u origen étnico.

Los datos disponibles hasta ahora sugieren que los déficits neurológicos persistentes observadas a los seis meses después del ictus incluyen: presencia de hemiparesia y déficit cognitivos en el 40-50\% y de hemianopsia, afasia y déficit sensorial en el 15-20\% de los pacientes que han sufrido un ictus ${ }^{28}$.

Las deficiencias estructurales y funcionales (fisiológicas) comprometen en diversos grados la realización de actividades y la participación de la persona, al provocar una o varias alteraciones motoras, sensoriales visuales ${ }^{29}$, sensoriales no visuales, sensitivas, cognitivas y del comportamiento. Por ejemplo, la afasia es una consecuencia común del accidente cerebrovascular, generalmente por lesiones del hemisferio izquierdo, estimándose que entre el 21 y el 38\% de los pacientes con accidente cerebrovascular sufren de afasia ${ }^{30}$. La presencia de afasia se ha asociado con una disminución general de respuesta a las intervenciones de rehabilitación del accidente cerebrovascular ${ }^{31}$. En el caso de la disfagia asociada al ictus, la incidencia publicada hasta la actualidad varía ampliamente del 19\% al 65\% en la fase aguda del ictus, dependiendo de la localización de la lesión, tiempo transcurrido desde la lesión y método diagnóstico utilizado ${ }^{31}$. A nivel 
emocional, el ictus afecta gravemente a la persona por lo que es muy habitual sufrir depresión ${ }^{14}$. En definitiva, el ictus repercute a nivel instrumental, provoca en muchas personas la dificultad o imposibilidad de realizar las actividades de la vida diaria.

Estas deficiencias producen daños a nivel laboral, educativo, social, médico, económico, entre otros. Las consecuencias de la discapacidad en adultos jóvenes en edad productiva generan un importante coste tanto personal, como familiar y social.

Lo que convierte al ictus en una de las afecciones que genera mayor carga social y económica $^{32}{ }^{33}$. Como afirma Schlesinger, la discapacidad genera un grave impacto social-psicológico en la persona adulta, pérdida de papel social, una desintegración de la identidad personal y las relaciones sociales ${ }^{34}$. Los pacientes con accidentes cerebrovasculares suelen estar afectados por discapacidades de larga duración con necesidades relativas a cuestiones sociales ${ }^{35}$. Por ello la rehabilitación integral es una buena inversión, ya que genera capacidad humana y reducción de daños.

Las secuelas que produce el ictus en todos los ámbitos de vida de la persona son de tal magnitud y relevancia para la persona que le sufre, su familia, el Sistema de Salud y por ende, la sociedad, que es necesario investigar que causas impiden que no reciba el tratamiento rehabilitador que precisa y contribuir a proporcionar autonomía funcional y mantenimiento de la persona en su entorno.

\section{LA REHABILITACIÓN INTEGRAL. CONCEPTO. DIMENSIÓN HOLÍSTICA E INTEGRAL DE LA PERSONA}

Entender la discapacidad es importante para poder acercarse a la rehabilitación desde el punto de vista teórico. No obstante, en la literatura encontramos el modelo integrador, el cual, integra el modelo médico y el modelo social, abordando la discapacidad como un problema dentro de la sociedad y no exclusivo del individuo. Este modelo integrador otorga importancia a la expresión y participación plena de todos los contextos ambientales capaces de influir positiva o negativamente sobre la persona en condición de discapacidad. Su manejo requiere la participación de elementos biológicos, psicológicos y las condiciones sociales 36373839

En el proceso de recuperación neurológica, estudiado por Edwarson ${ }^{28}$ entre las 12 horas y los 7 primeros días tras un ictus isquémico, muchos pacientes sin complicaciones comenzaron a mejorar de sus déficits neurológicos; produciéndose la mayor recuperación de éstos entre los primeros 3 a 6 meses. La discapacidad a los seis meses después del 
ACV se asoció con la aparición de depresión, incapacidad para caminar sin ayuda y deficiencias sociales en un $30 \%$, con la necesidad de atención institucional en un $25 \%$ y con la presencia de incontinencia urinaria en un $22 \%$ de los $\operatorname{casos}^{28}$. El estudio de Edwarson demostró que algunos pacientes continuaron mejorando hasta los 18 meses. Sin embargo, esta discapacidad asociada en un 30\% a deficiencias sociales, no está siendo cubierta. En un estudio ${ }^{35}$ que se realizó con 57 pacientes, el 30\% de los pacientes nunca había recibido apoyo social profesional. El contacto profesional con trabajo social se produjo principalmente durante la estancia en el Hospital de agudos o en la institución de rehabilitación. Solo cuatro pacientes (11\%) informaron de un apoyo a largo plazo tras el alta. El 43\% de los pacientes tenían necesidades no satisfechas en los cuidados posteriores a largo plazo. Se demostró que las necesidades sociales no satisfechas se asociaban a una menor calidad de vida y a una mayor carga para los cuidadores ${ }^{35}$.

En un estudio nacional de necesidades, oferta y demanda de servicios de rehabilitación, realizado en Colombia en $2003^{40}$, para identificar vacíos en el conocimiento de la realidad de la rehabilitación se observó que la mayoría de las intervenciones quedan cortas frente a la integración de las personas al entorno a su plena y efectiva participación en la sociedad $^{41}$, los servicios se orientan fundamentalmente al mejoramiento de funciones y estructuras corporales.

Por ello la rehabilitación no debe limitarse a la mejora funcional, sino que debe incluir la participación e integración de la persona en la sociedad. Este tipo de rehabilitación integral de la discapacidad producida por un ictus es un asunto que concierne a toda la sociedad y al sector de salud como elemento fundamental y necesario para su logro. Albrecht realiza una afirmación que será destacada de ahora en adelante a efectos de esta investigación: la rehabilitación es una respuesta al problema social de la discapacidad ${ }^{42}$. Sin embargo, se tiene relativamente poco en cuenta la recuperación social de los pacientes y el apoyo necesario para volver al trabajo, recibir prestaciones sociales, participar en las actividades de la vida diaria, mantener contacto con la familia y amigos ${ }^{35}$ y permanecer en su entorno. La sociedad pone muchas limitaciones para prestar servicios apropiados a las personas con discapacidad, no les presta y no son cubiertas sus necesidades ${ }^{43}$.

\section{1. ¿Qué es la Rehabilitación Integral?}

En el año 2004, Boada ${ }^{44}$ retoma el ejercicio de reflexión en torno a la rehabilitación y hace un análisis a profundidad del concepto de rehabilitación integral, donde expone que tal término tiene sus orígenes en la concepción biopsicosocial de los individuos; pese a 
que a lo largo del tiempo no ha perdido su connotación médica al partir del déficit. Sus consideraciones apuntan, a reflexionar sobre la discapacidad, más precisamente desde la perspectiva del enfoque de capacidades para dirigir las acciones hacia el logro de la inclusión ${ }^{44}$.

La rehabilitación integral considera que la persona es un ser bio-psico-social, y que, a través de procesos terapéuticos, educativos, formativos y sociales, se logra mejorar la calidad de vida y la plena integración de la persona al medio familiar, social y ocupacional $^{45}$, desarrollando su máximo potencial físico, psicológico y social ${ }^{45}$. Así pues, la rehabilitación integral atiende la totalidad de las áreas afectadas de la persona, pues trabaja aspectos físicos, cognitivos y sociales, con el fin de conseguir la máxima independencia funcional posible, promover la salud y mejorar la calidad de vida del paciente.

Se basa en la capacidad y experiencia de un equipo interdisciplinar que aborda la asistencia del paciente de manera integral. Ese equipo multidisciplinar integrado por diferentes profesionales competentes en su área de intervención será el responsable de su proceso asistencial, aunque muchos otros profesionales intervengan lo largo de todo el proceso $^{45}$. Los programas de rehabilitación multidisciplinar y coordinada han demostrado no solo disminuir la mortalidad de estos pacientes sino también mejorar la funcionalidad,

el grado de participación y la calidad de vida de pacientes y cuidadores ${ }^{46}$. Su evaluación e intervención han de ser precoces para optimizar la rehabilitación.

La presencia del equipo multidisciplinar exige que todo paciente sea valorado por parte de todos los miembros del equipo con el objetivo de plantear un abordaje terapéutico común que incluya metas de trabajo coordinadas, precisas, medibles, relevantes, realistas y alcanzables.

\subsubsection{Modelo holístico}

El enfoque holístico y otros enfoques de carácter integral se caracterizan por la alta estructuración e integración entre las intervenciones de diferente índole (cognitiva, emocional, funcional, física, social, etc.), lo que demanda una coordinación intensiva entre los diferentes integrantes del equipo transdisciplinar, el paciente y la familia. Este tipo de enfoques ha demostrado una mayor eficacia por su carácter estructurado y coordinado 47 .

Este modelo de rehabilitación aboga por implicar activamente a pacientes, familiares y cuidadores como miembros esenciales del equipo de rehabilitación e imprescindibles en 
la toma de decisiones, la aceptación social y el mantenimiento de las ganancias obtenidas con el tratamiento.

Este modelo es una alternativa para brindar una atención integral aprovechando los recursos personales, comunitarios y del sector público; con una visión transformadora; con la posibilidad de tener una vida digna y equitativa desde la inclusión, el apoyo, la autodeterminación y las capacidades para desarrollarse como persona autosuficiente ${ }^{38}$. El ictus provoca en cada persona unas secuelas concretas y afectación en áreas específicas, además la persona tiene una determinada familia y una situación socioeconómica propia y única. La singularidad de cada caso, implica el tratamiento individualizado e integral del mismo. Se requiere de atención integral profesionalizada donde las diferentes disciplinas aporten elementos para construir el diagnóstico y el plan de tratamiento 4849 ${ }^{50}$. La rehabilitación personalizada integral por equipo interdisciplinar es el tratamiento más efectivo para dar respuesta a sus necesidades, una atención de profesionales formados en el dominio afectado y experimentados en esta patología, que incluye al paciente y familia. Este modelo organizativo basado en un equipo interdisciplinar experto, en colaboración con pacientes y familiares, ha demostrado conseguir los mejores resultados en la rehabilitación del ictus ${ }^{47}$.

\subsection{El proceso de Rehabilitación Integral del ictus}

Valero en su ponencia Modelo de rehabilitación integral, presentada en el primer Congreso Internacional de rehabilitación integral, plantea la rehabilitación integral de las personas en condición de discapacidad como un sistema abierto con el objetivo principal de "integrar progresivamente a algunos de sus miembros a la vida productiva". Sus desarrollos dividen la rehabilitación por procesos, separando el ámbito funcional, profesional y social ${ }^{38}$.

Estos ámbitos son entendidos por algunos autores como diferentes etapas a desarrollar ${ }^{38}$, de tal manera que el logro de cada una de ellas garantiza la rehabilitación integral. Es decir, la rehabilitación integral se basa en el logro de ${ }^{38}$ :

$1^{\text {a) }}$ La rehabilitación funcional: promueve el logro de la máxima capacidad física, mental y psicológica, va determinando por el equipo interdisciplinar de manera continua y oportuna el logro de recuperación de las áreas funcionales afectadas;

$2^{\text {a }) ~ L a ~ r e h a b i l i t a c i o ́ n ~ p r o f e s i o n a l: ~ s e ~ o r i e n t a ~ h a c i a ~ l a ~ i n c l u s i o ́ n ~ d e ~ l a ~ p e r s o n a ~ e n ~ s u ~}$ medio, promoviendo la orientación, formación, adaptación y seguimiento para lograr favorecer su propia autonomía, a pesar de presentar déficits funcionales; 
$3^{\text {a) }}$ La rehabilitación social, es la etapa final de la rehabilitación integral, favoreciendo la integración del individuo en la sociedad, es decir, su plena inclusión, mantenimiento en su entorno.

Es evidente, que el logro de la rehabilitación integral pasa indudablemente por el acceso a una rehabilitación funcional, adecuada, coherente y estructurada, teniendo en cuenta que, aunque su objetivo es la recuperación del paciente, si persisten secuelas no sea privado de una rehabilitación integral que contribuya a su reinserción socio-profesional Considerando nuestra experiencia profesional en rehabilitación de pacientes con ictus, desarrollada en el Servicio de Neurología del HCUV, el proceso de rehabilitación integral requiere atender y considerar los aspectos profesionales y sociales durante el tratamiento rehabilitador funcional, por lo que no debería hablarse de fases, sino de componentes articulados entre sí, que se retroalimentan unos con otros, durante todo el proceso de rehabilitación integral, que abarca tanto el período de ingreso hospitalario, como los tratamientos rehabilitadores (funcionales y sociales) post-alta hospitalaria.

Los aspectos por considerar en un proceso de rehabilitación integral de pacientes que han sufrido un ictus se detallan a continuación, junto con la finalidad de la rehabilitación integral: recuperación funcional y reinserción socio profesional.

\subsubsection{Componente funcional}

El componente funcional de una rehabilitación integral es el proceso de acciones médicas y terapéuticas, encaminadas a lograr que las personas con discapacidad estén en condiciones de alcanzar y mantener un estado funcional óptimo desde el punto de vista físico, sensorial, intelectual, psíquico o social, de manera que les posibilite modificar su propia vida y ser más independientes ${ }^{41}$.

Se trata de un tipo de rehabilitación que debe recibir un paciente que haya sufrido un ictus, en el que se constate una pérdida de las capacidades físicas, cognitivas, sensoriales, emocionales, conductuales y/o funcionales (estructuras y funciones), con repercusión en el grado de actividad y/o participación de la persona, debiéndose prescribir una vez se haya alcanzado una mínima estabilidad clínica y se hayan controlado las posibles complicaciones que afecten al estado vital del paciente ${ }^{47}$.

A pesar de ello, una gran cantidad de pacientes con necesidades de rehabilitación funcional (cuyos déficits son rehabilitables) no son derivados a servicios de rehabilitación. Un estudio comparativo reciente evaluó las necesidades de rehabilitación: menos de la mitad de los pacientes que habían sufrido un ictus en Australia tuvieron una 
valoración de sus necesidades de rehabilitación, frente a un 58\% de pacientes canadienses, un $89 \%$ de pacientes británicos y el $90 \%$ de los pacientes estadounidenses ${ }^{51}$. En nuestro país no existen datos que puedan ser comparables, si bien la última auditoría del Pla Director de la Malaltia Vascular Cerebral, realizado sobre el $18.7 \%$ de los pacientes ingresados por ictus en Cataluña en 2013, mostró que hasta el $49.8 \%$ de los supervivientes presentaban objetivos rehabilitables ${ }^{52}$.

El inicio precoz es clave en el proceso de recuperación funcional. Junto con la terapia intensiva orientada a tareas mejoran los resultados funcionales tras el ictus. La intensidad del programa de rehabilitación del paciente, así como el medio en el que se realiza el tratamiento de rehabilitación son predictores importantes de los resultados del paciente tras el ictus ${ }^{46}$. Es fundamental, que todo paciente hospitalizado tras sufrir un ictus agudo sea sometido a una valoración inicial por expertos en rehabilitación lo más pronto posible desde su ingreso hospitalario. Recibiendo la rehabilitación prescrita tan pronto como se estime que están preparados para ello y se encuentren lo suficientemente estables desde el punto de vista médico como para participar activamente en programas de rehabilitación ${ }^{47}$. Demasiados pacientes abandonan el hospital sin que se haya iniciado la rehabilitación, en muchos hospitales porque carecen de estos profesionales especializados o porque no hay establecido una coordinación eficaz y eficiente. En otras ocasiones el paciente abandona el hospital y tarda días en iniciar el tratamiento rehabilitador por no disponibilidad del recurso al alta médica. Esto resulta especialmente preocupante para los esquemas de alta precoz con rehabilitación ambulatoria ${ }^{31}$, debido a que el inicio precoz tiene relación con la disponibilidad del recurso, posibilidad de acudir el paciente o familia, posibilidad de pagar el tratamiento, etc.

Los pacientes deben recibir tratamiento rehabilitador de intensidad y duración apropiadas, diseñados individualmente para satisfacer los mayores objetivos de recuperación y teniendo en cuenta sus niveles de tolerancia. Con la frecuencia y duración que sean capaces de participar y mostrar un beneficio medible del tratamiento.

Más terapia produce mejores resultados, pero no todos los tratamientos terapéuticos que precisa el paciente están subvencionados por los Sistemas Públicos de Protección, y tampoco están disponibles geográficamente o a su alcance económico. El acceso al tratamiento rehabilitador debe mejorarse según avala un informe presentado al Parlamento Europeo ${ }^{53}$.

El equipo multidisciplinar responsable de este tratamiento comprende los siguientes profesionales con experiencia en rehabilitación funcional del ictus: médicos, enfermería, 
fisioterapeutas, terapeutas ocupacionales, logopedas, psicólogos clínicos, trabajador/a social y auxiliares de enfermería. En la guía de práctica clínica de rehabilitación del ictus, publicada en 2016 por Hebert y colaboradores ${ }^{54}$, el paciente y la familia también son incluidos como parte del equipo principal.

La decisión acerca del potencial de recuperación ha de recaer en este equipo, empleando herramientas validadas, estandarizadas, y adaptadas a la gravedad clínica.

Un equipo interdisciplinar de profesionales de la salud con experiencia en el cuidado del paciente que ha sufrido un ictus, independientemente del lugar donde se preste el servicio, con el fin de asegurar la consistencia del tratamiento y disminuir el riesgo de complicaciones.

Además, los espacios de tratamiento deben ser espacios individualizados, dedicados específicamente al tratamiento de estos enfermos, con posibilidad de acceso a otros servicios (ortopedia, farmacia, nutrición, etc.) y contar con un programa educativo multidisciplinar dirigido a las familias ${ }^{47}$.

La valoración por áreas de función es necesaria al tratarse de factores que intervienen en las relaciones sociales, familiares de los pacientes, influyen en mantener la comunicación, las relaciones interpersonales, el seguimiento de pautas y son necesarias de identificar para el logro de la rehabilitación integral ${ }^{14}$. Por eso es fundamental una valoración multidisciplinar para una correcta intervención y manejo del paciente.

El equipo interdisciplinar valorara el tratamiento en los siguientes dominios, que aparecen en la Tabla 1: 
Tabla 1. Áreas afectadas por ictus y tratamiento

\begin{tabular}{|c|c|}
\hline Áreas afectadas & Tratamiento \\
\hline $\begin{array}{l}\text { 1.Área Motora y discapacidad } \\
\text { física } \\
\text { Es frecuente que el ictus afecte al } \\
\text { movimiento, la hemiparesia o } \\
\text { hemiplejia constituye el déficit más } \\
\text { prevalente tras un ictus por lo que es } \\
\text { preciso incluir la valoración del área } \\
\text { motor }\end{array}$ & $\begin{array}{l}\text { Los objetivos de la recuperación motora se } \\
\text { centran en restaurar el control motor de la } \\
\text { deambulación, mejorar la función del } \\
\text { miembro superior y aumentar la } \\
\text { participación en general. }\end{array}$ \\
\hline $\begin{array}{l}\text { 2.Lenguaje y comprensión } \\
\text { Afectación del lenguaje en sus } \\
\text { vertientes de comprensión, } \\
\text { denominación, repetición, lectura y } \\
\text { escritura }^{14}\end{array}$ & $\begin{array}{l}\text { La reintegración de la habilidad para el } \\
\text { habla, la fluidez, la comprensión, la lectura y } \\
\text { escritura es objetivo prioritario. } \\
\text { AFASIA: Se recomienda que todo paciente } \\
\text { con problemas de comunicación después de } \\
\text { un ictus reciba tratamiento logopédico, } \\
\text { adaptado individualmente a sus necesidades. }\end{array}$ \\
\hline $\begin{array}{l}\text { 3.Deglución } \\
\text { El paciente puede presentar signos de } \\
\text { disfagia }\end{array}$ & $\begin{array}{l}\text { La guía NICE de } 2013^{55} \text { recomienda terapia } \\
\text { de deglución al menos } 3 \text { veces a la semana a } \\
\text { las personas con disfagia después del } \\
\text { accidente cerebrovascular que son capaces } \\
\text { de participar, mientras continúen } \\
\text { consiguiendo mejoras funcionales. }\end{array}$ \\
\hline $\begin{array}{l}\text { 4.Perceptiva y cognitiva } \\
\text { Así pues, en el dominio cognitivo, el } \\
\text { ictus puede ocasionar daños en la } \\
\text { memoria, atención, orientación, } \\
\text { habilidades de cálculo, habilidad de } \\
\text { aprender }^{14} \text {. }\end{array}$ & $\begin{array}{l}\text { Se debe evaluar a las personas después del } \\
\text { accidente cerebrovascular para detectar } \\
\text { déficits cognitivos. Cuando se identifica un } \\
\text { déficit cognitivo, debe realizarse una } \\
\text { evaluación detallada utilizando herramientas } \\
\text { válidas, confiables y receptivas antes de } \\
\text { diseñar un programa de tratamiento }{ }^{55} \text {. }\end{array}$ \\
\hline $\begin{array}{l}\text { 5.Sensitiva y visual } \\
\text { El ictus también puede provocar } \\
\text { déficits sensitivos o visuales }\end{array}$ & $\begin{array}{l}\text { Se debe evaluar a las personas para } \\
\text { detectarlas. Se destaca la falta de tratamiento } \\
\text { rehabilitador visual garantizado por el } \\
\text { Sistema Público de Salud. }\end{array}$ \\
\hline
\end{tabular}

Elaboración propia a partir de ${ }^{1431}$ Arias A. y Sánchez Tarifa MP.

La valoración de las secuelas de un ictus no ha de limitarse a describir las alteraciones en cada dominio por sí mismas, sino que hay que valorar la repercusión funcional en la persona y en sus actividades dentro de su entorno ${ }^{14}$. 
Es necesario el tratamiento de todas las áreas formando parte del tratamiento integral, coordinado, coherente y complementado con las otras terapias que pueda necesitar, debiendo ser evaluado periódicamente por un equipo multidisciplinar.

Los datos son desalentadores: en toda Europa hay especial falta de terapia ocupacional, logopedia y psicoterapia $^{53}$ y no hay trabajo social especializado en neurología o neurorrehabilitación.

Además, la depresión que repercute en el estado funcional del paciente que aparece post ictus, no está siendo tratada en la mayoría de $\operatorname{los} \operatorname{casos}^{13}$, no se evalúa de forma rutinaria después del ictus, y por ello, sólo una minoría son diagnosticados y tratados correctamente ${ }^{56}$.

El medio o centro donde proporcionar esta rehabilitación funcional tiene que determinarse respetando la autonomía y decisión del paciente, y no siempre existen recursos para que sea posible.

Se deben proporcionar servicios de rehabilitación de acuerdo con las recomendaciones establecidas en la guía antes citada ${ }^{47}$ :

- Una unidad hospitalaria de rehabilitación de ictus capaz de proporcionar rehabilitación a todo paciente hospitalizado que haya sufrido un ictus. No existen estas unidades en todos los hospitales y cabe destacar que dentro del equipo de rehabilitación del ictus no todos cuentan con trabajo social neurológico, terapia ocupacional, ni logopedia. Existen hospitales que no cuentan con estos profesionales: "Durante la hospitalización, al carecer de logopeda en nuestro Hospital, el paciente no recibió tratamiento logopédico" ${ }^{30}$. Cabe destacar que dentro del equipo de rehabilitación del ictus tampoco aparece la figura del psicólogo ${ }^{56}$. En la guía de práctica clínica de rehabilitación del ictus publicada en 2016 por Hebert y cols., se demuestra que la rehabilitación coordinada y organizada en una unidad de ictus aumenta la independencia funcional y la calidad de vida $^{54}$, siempre que cuente con los profesionales competentes para atender cada dominio afectado.

Al alta hospitalaria del Hospital de Agudos (desde unidad de ictus o desde planta), entre las recomendaciones de la guía canadiense en $2016^{54}$, se establece que los supervivientes del ictus con objetivos rehabilitables deben continuar teniendo acceso a servicios especializados después de abandonar el hospital. Esos servicios deben incluir servicios de rehabilitación en casa, en centro residencial o ambulatorio. El objetivo es conseguir la máxima independencia funcional y autonomía en los actos de la vida cotidiana y 
permanencia en domicilio.

- Rehabilitación domiciliaria precoz para facilitar que toda persona con ictus pueda recibir rehabilitación en su domicilio.

El equipo multidisciplinar de rehabilitación puede desplazarse al domicilio del paciente cuando las condiciones médicas o sociales no permiten el desplazamiento de éste al centro de rehabilitación.

Está indicada en pacientes con discapacidad moderada/grave, que cuentan con apoyo sociofamiliar suficiente para poder regresar a su domicilio habitual, con condiciones médicas y cognitivas que le permitan participar en un programa de rehabilitación, pero que no pueden desplazarse a un centro ambulatorio de rehabilitación (hospital o centro concertado) ${ }^{57}$. Estos programas de rehabilitación a domicilio se ha demostrado que reducen la estancia hospitalaria y mejoran la capacidad funcional en los pacientes con ictus moderados-graves ${ }^{58}$.

- Rehabilitación en centros especializados con carácter residencial capaces de cubrir las necesidades de salud, sociales y laborales/educativas de pacientes con ictus de cualquier edad.

- Rehabilitación ambulatoria especializada en los servicios hospitalarios u otros centros especializados. Para aquellos individuos clínicamente estables, sin déficits cognitivos importantes, con discapacidad leve/moderada en una o dos áreas funcionales, con adecuado soporte sociofamiliar y con condiciones médicas y cognitivas que les permitan desplazarse al centro de rehabilitación ${ }^{57}$.

En conclusión, los pacientes con discapacidad después de un ictus deben recibir rehabilitación en unidades especializadas para pacientes con ictus frente a servicios de rehabilitación convencionales ${ }^{59}$. Estos servicios deben incluir tanto programas domiciliarios asociados a los programas de alta domiciliaria precoz como programas de rehabilitación en centros ambulatorios especializados. Tienen que estar garantizados por la especialización del equipo interdisciplinar y la coordinación entre proveedores de la neurorrehabilitación entre sí y con el paciente y familia, como elementos fundamentales para una mayor eficacia del tratamiento. El tratamiento dispensado por equipos multidisciplinares especializados, en un solo emplazamiento, y con los objetivos terapéuticos consensuados entre el equipo, paciente y familiares, resulta más eficaz a nivel de promoción de la independencia funcional (tanto en actividad, como en participación) que el tratamiento dispensado por servicios aislados, que no son especialistas en neurorrehabilitación, ni se coordinan entre $\mathrm{sí}^{47}$. El inicio y la intensidad 
del programa de rehabilitación del paciente, así como el medio en el que se realiza el tratamiento de rehabilitación también, son predictores importantes de los resultados del paciente tras el ictus ${ }^{46}$. El retraso en el inicio del tratamiento rehabilitador al alta hospitalaria se asocia con una disminución de la reorganización cortical y una reducción de las posibilidades de alcanzar una adecuada recuperación funcional ${ }^{6061}$. Así, cuanto mayor sea la gravedad inicial del ictus, más son las funciones que hay que recuperar, por lo que el proceso de neurorrehabilitación precisaría de más tiempo ${ }^{6061}$.

Por ello, al alta de estos programas activos de rehabilitación, es recomendable mantener controles periódicos con los sistemas sanitarios comunitarios y si es necesario con los miembros del equipo de tratamiento, con el objetivo de realizar controles de salud y hábitos saludables, detección de posibles complicaciones o cambios en la autonomía funcional. Siempre teniendo en cuenta, que si en cualquier momento a lo largo del proceso de recuperación tras sufrir un ictus, el paciente experimenta un cambio en su estado funcional susceptible de mejoría con programas de rehabilitación adicionales, se les debería ofrecer un programa adicional de rehabilitación ambulatoria ${ }^{47}$.

Es importante destacar que, en base a la situación personal y los déficits persistentes tras el proceso de rehabilitación funcional, se establecen e identifican prioridades de acción, se definen metas a alcanzar y se posibilita el seguimiento, para su inserción socioprofesional.

\subsubsection{Componente profesional}

El componente profesional de la rehabilitación integral es un proceso encaminado a lograr que una persona compense en el mayor grado posible las habilidades y destrezas afectadas por una condición de discapacidad que repercute directamente en su desempeño profesional $^{62}$. Se trata de un proceso continuo de adaptación y readaptación que comprende básicamente las etapas de orientación, formación profesional y colocación selectiva para que la persona con discapacidad se capacite y logre de esa forma integrarse o reintegrarse a la vida socio productiva ${ }^{6263}$.

Este componente del proceso de rehabilitación integral es un medio para lograr la plena inclusión social del paciente, por lo que debe responder al compromiso último de insertar productivamente a la persona que tras una rehabilitación funcional queda con alguna discapacidad en su contexto socio-profesional, atendiendo para ello los más elevados estándares de rendimiento, calidad y satisfacción personal.

Diferentes autores han destacado la urgencia de promover iniciativas específicas que 
faciliten el logro y desempeño de un trabajo por las personas que sufrieron daño cerebral traumático, como unos de los recursos que favorecen su independencia y permiten una mejor calidad de vida ${ }^{64} 65$.

En este aspecto, no debemos olvidar que las personas con discapacidad, "muchas veces no pueden acceder a puestos de trabajo por su propia discapacidad y en otras ocasiones no acceden al empleo por la discriminación que sufren en el mercado laboral, independientemente de sus capacidades y de su formación para el puesto de trabajo"66. Por ello, el componente profesional-laboral de la rehabilitación va inextricablemente unido al aspecto social y convivencial. El trabajo es una poderosa herramienta de socialización que proporciona no sólo ingresos económicos (bajo forma de empleo remunerado), sino también identidad, actividad, capacidad para estructurar el tiempo y relaciones interpersonales ${ }^{66}$. Por ello, independientemente de que se pueda obtener y/o recuperar el empleo, la rehabilitación integral ha de atender los aspectos de sociabilidad del paciente, para evitar que su situación de vulnerabilidad tras el ictus, le lleve a una situación de exclusión.

La promoción del logro y desempeño de un trabajo, de integrarse o reintegrase a la vida productiva, es uno de los recursos más positivos que favorecen su independencia y permiten una mejor calidad de vida.

\subsubsection{Componente social}

Es sabido, que los pacientes que han sufrido ictus suelen estar afectados por discapacidades de larga duración con necesidades relativas a cuestiones sociales. El ictus perjudica el equilibrio y la capacidad de adaptación social. Sin embargo, se tiene relativamente poco en cuenta la recuperación social de los pacientes y el apoyo necesario para volver al trabajo, recibir prestaciones sociales, participar en las actividades de la vida diaria, mantener contacto con familia y amigos ${ }^{35}$.

La sociedad pone muchas limitaciones para prestar servicios apropiados a las personas con discapacidad, no les presta estos servicios y no son cubiertas sus necesidades ${ }^{43}$. En las últimas décadas, hemos asistido a una nueva situación que se manifiesta haciendo que las personas, familias y grupos que antes se encontraban en situación de inclusión puedan pasar a estar excluidos de forma angustiosamente rápida e imprevisible. Anteriormente este proceso era mucho más lento, y por contra, actualmente este proceso puede derivarse en apenas unos días. En la sociedad actual, todo se ha vuelto más rápido, también los procesos de inclusión-exclusión social. Se debe evaluar el grado de participación e 
integración del sujeto en su comunidad, con el objetivo de garantizar que se están abordando las necesidades personales de cada paciente.

En definitiva, la rehabilitación integral es la integración de estos tres componentes (funcional, profesional y social), garantiza el mejoramiento de la calidad de vida y la plena integración al medio familiar, social y ocupacional, a través de procesos terapéuticos, educativos y formativos que se brindan acorde al tipo de discapacidad ${ }^{41}$ que pueda presentar la persona tras finalizar su rehabilitación funcional.

En el tratamiento rehabilitador integral, la educación, información, capacitación y acceso a servicios necesarios de las personas que han sufrido el ictus, sus familias y cuidadores deben estar presentes, especialmente en los momentos de transición de un tratamiento rehabilitador a otro, con el objetivo de que este alcance el máximo grado de actividad y participación social ${ }^{52}$. Incorporando y valorando en el proceso de rehabilitación de la persona, su capacidad para afrontar la nueva situación generada, el riesgo de depresión y otros problemas físicos y psicológicos que puedan presentar y ofrecerles el tratamiento requerido.

Por ello, el equipo rehabilitador debe ${ }^{47}$ contar con formación especializada en el cuidado y la recuperación del paciente con ictus; debiendo acreditar formación específica y la suficiente experiencia clínica en la rehabilitación del paciente con daño cerebral adquirido (DCA) que les capacite para evaluar los distintos aspectos socio sanitarios que pueden acontecer a lo largo del curso clínico de la enfermedad:

- La capacidad de abordar tanto los problemas físicos como cognitivo-conductuales y sociales (estructura función-actividad y participación) que el paciente pueda presentar a lo largo de todo el proceso de recuperación.

- Con el conocimiento, las habilidades y la predisposición para trabajar en equipo con personas que han sufrido un ictus y sus familias y con capacidad para asimilar y manejar los cambios-necesidades que se producen como consecuencia de un ictus.

Los servicios en rehabilitación deben centrar sus esfuerzos en equiparar la serie de capacidades trabajando, no solo desde las estructuras, funciones y actividades, sino removiendo las restricciones en la participación, favoreciendo la autonomía de los individuos ya que establecer metas solo en la capacidad para funcionar, sin evaluar las capacidades de la persona, obvia la oportunidad de proporcionar un enfoque de derechos al acto de rehabilitar, caso específico el derecho fundamental de vivir la vida que se quiere 1levar ${ }^{67}$. 


\subsection{Finalidad y beneficios de la rehabilitación integral post-ictus}

La rehabilitación integral es una parte esencial de los cuidados post-ictus, que está orientada a alcanzar el mejor pronóstico funcional e integración familiar, social y profesional del paciente. Debe contribuir a que todas las personas tengan la posibilidad real y efectiva de acceder, participar, relacionarse y disfrutar de un bien, servicio o ambiente, junto con los demás ciudadanos, sin ninguna limitación o restricción por motivo de discapacidad, mediante accesiones concretas que ayuden a mejorar la calidad de vida de las personas con discapacidad ${ }^{41}$. La OMS no desconoce esta meta, y define la participación social como uno de los fines de los servicios sociales y de salud.

Seelman, en su artículo Tendencias en rehabilitación y discapacidad: Transición de un modelo médico a un modelo integral, hace una exploración de los modelos de discapacidad vigentes y su operacionalización en el contexto de los países que históricamente los han implantado ${ }^{36}$, concluyendo su recorrido en la rehabilitación integral como un modelo que responde de mejor manera a las necesidades de las personas con discapacidad. Según esta autora, la rehabilitación integral intenta disminuir la brecha entre profesiones, disciplinas y pacientes, argumentando que la persona con discapacidad desempeña diferentes roles en la sociedad incluyendo el de ciudadano y paciente, entre muchos otros.

Es evidente, que el modelo tradicional de rehabilitación no aborda con las personas cuestiones como el problema de desintegración social, la pérdida de estatus y relaciones sociales, entre otros, que una rehabilitación integral sí contempla y cuya atención a estos aspectos sí garantiza la recuperación integral del paciente. Estas actuaciones deben ser atendidas durante el proceso para no influir e impedir el avance y recuperación de otras áreas afectadas y, además, deben ser un fin que garantice la rehabilitación integral (conservar puesto de trabajo, relaciones sociales, permanencia en el hogar, etc.).

A la vista de estos datos, caben formularse varios interrogantes: ¿Comprendemos bien las limitaciones en la actividad de nuestros pacientes que sufren ictus? ¿Comprendemos o tenemos certeza sobre las restricciones de su participación en la vida social en nuestros pacientes con ictus? ¿Se está garantizando una rehabilitación que contribuya no solo a disminuir la discapacidad que provoca el ictus, sino también, cuando esto no sea posible, a su integración en la sociedad de estos pacientes?

Lograr el fin último de la rehabilitación integral, la integración de la persona en la sociedad resulta inviable con las dificultades a las que nos enfrentamos para que los 
pacientes accedan a la misma. En síntesis, las dificultades más frecuentes para acceder a la rehabilitación integral son las siguientes:

- Inexistencia de servicios especializados públicos en todas las localidades donde residen los pacientes que sufren ictus, ni recorriendo distancias cortas (menos de 100 kilómetros).

- Los centros especializados de rehabilitación públicos establecen criterios de exclusión, como la edad, a pesar de que el paciente sea rehabilitable.

- Los centros especializados privados no están al alcance, ni geográfica ni económicamente, de todas las personas que sufren ictus.

- No todos los miembros del equipo profesional rehabilitador están especializados (entre ellos el/la trabajador/a social).

- Dificultad para el inicio precoz del tratamiento rehabilitador

- Ausencia de seguimiento del proceso rehabilitador para facilitar la transición de un recurso a otro, si el paciente sigue manteniendo criterios rehabilitables.

- Escasa o nula participación de paciente y/o familia en el proceso rehabilitador.

- Dificultades para seguir el tratamiento rehabilitador sin abandonar las responsabilidades familiares.

- Imposibilidad de rehabilitación domiciliaria o ambulatoria por barreras arquitectónicas en la vivienda o sus condiciones insalubres.

Estas dificultades implican predominio de la discapacidad y no superación de la recuperación de las áreas funcionales del paciente, fase clave para el logro de una mayor integración y participación de la persona en la sociedad, mantenimiento en su domicilio, reincorporación a la vida profesional.

Por ello, es necesario investigar sobre los factores socioambientales y económicos que influyen en el acceso a la rehabilitación integral y en el pronóstico de recuperación de la persona, para el logro de su mayor calidad de vida e integración social.

\section{IMPORTANCIA DE LOS FACTORES PERSONALES Y SOCIOFAMILIARES EN EL ACCESO REHABILITACIÓN INTEGRAL POST-ICTUS Y EN SU PRONÓSTICO DE RECUPERACIÓN}

El acceso a un tratamiento rehabilitador integral es fundamental para disminuir la discapacidad y mejorar la funcionalidad del paciente que ha sufrido un ictus ${ }^{30} \mathrm{y}$ por 
consiguiente, posibilitar con mayor garantía/éxito/eficacia su reinserción social y profesional (dado que a menor cantidad de secuelas, mayores son las posibilidades de reinserción laboral y social). Se debe tener presente y no obviar las dificultades que existen en el Sistema de Salud y en las personas-familias para poder recibirla, por ello, para que pueda hacerse una realidad el acceso a la misma, abordaremos diferentes factores que inicialmente se consideran condicionantes del acceso a la rehabilitación funcional integral. Es necesario su conocimiento y análisis. En el año $2015^{68}$ teniendo presente que el coste de la hospitalización para el ictus era alto, y que sería aconsejable considerar destino al alta hospitalaria de forma precoz y seleccionar a los pacientes para su destino. Se publicó una revisión sistemática sobre los factores pronósticos para el destino al alta después del ictus concluyendo en que la atención médica inicial, la edad, el sexo, las complicaciones neurológicas y médicas y los factores ambientales/socioeconómicos se debían considerar en el proceso de toma de decisiones para el destino al alta hospitalaria puesto que una mejor predicción del destino al alta podría conducir a una longitud más corta de la estancia en cuidados intensivos, mejor evolución del paciente, reducción de costes y mejor preparación de los cuidadores informales ${ }^{68}$. Es innegable que el hombre es un ser influenciado por el ambiente social y económico en que vive ${ }^{69}$.Además, es una realidad que no existen recursos de rehabilitación funcional integral al alcance de todas las personas que son rehabilitables. En un metaanálisis reciente se consideró como factores negativos de acceso a recursos de rehabilitación ${ }^{70}$ : la presencia de una discapacidad funcional o cognitiva premórbida, la edad ( $>70$ años) y la ausencia de apoyo familiar. En la literatura actual existe una diversidad de criterios de derivación a centros de rehabilitación basada fundamentalmente en aspectos personales, estructurales o económicos ${ }^{71} 72$ En un reciente estudio ${ }^{71}$ las condiciones más justificables por las que los médicos responsables de los casos de discapacidad resultante después de una lesión cerebral consideraban su derivación a sistemas de neurorrehabilitación fueron: Situación cognitiva premórbida, nivel comunicativo premórbido y movilidad premórbida. Mientras que las causas de exclusión más frecuentemente empleadas para denegar el acceso a estos recursos fueron: La movilidad post-ictus, el apoyo social y la situación cognitiva postictus $^{71}$.

En definitiva, actualmente los procesos de triaje, están expuestos a la susceptibilidad individual de la persona u órgano responsable del mismo en función de aspectos económicos, personales, estructurales y actitudinales. Aun disponiendo de criterios objetivos de selección, el propósito de la selección puede ser muy variable, en función 
por ejemplo de la financiación disponible, los recursos asistenciales existentes o la proximidad al domicilio de estos, $\mathrm{u}$ otras prioridades personales/institucionales en base a criterios muy diversos ${ }^{73}$.

Todo ello hace que las variables determinadas hasta la fecha para la selección de candidatos a neurorrehabilitación incluyan pacientes no apropiados y excluyan pacientes apropiados. Ante este escenario, varios autores han propuesto que probablemente el mejor criterio que determine la capacidad para beneficiarse de la rehabilitación sea la propia demostración de beneficio una vez estos han sido incluidos dentro del propio programa que se considere oportuno, asegurando que aquellos pacientes no admitidos son atendidos por los recursos de apoyo necesarios ${ }^{74}$, esto es lo que se ha querido comprobar en la investigación realizada para esta tesis. Actualmente, es indudable que existen factores de riesgo no modificables que influyen en la aparición de ictus, tales como edad, sexo, entre otros, se considera y pretende averiguar que también influyen en el acceso a la rehabilitación integral. Las estrategias para mejorar el acceso a la rehabilitación de pacientes que sufren ictus deben abordar la identificación y conocimiento de estos, para desarrollar alternativas que frenen el efecto negativo del factor en la rehabilitación integral.

\subsection{Factores personales}

La persona presenta unas cualidades y capacidades que le hacen única, con sus singularidades y diferencias. Para la atención a las necesidades originadas por el ictus en los pacientes no existen fórmulas únicas ni sirven tratamientos generales, es imprescindible, tener presente la singularidad de cada persona. El tratamiento rehabilitador postictus tiene que ser individualizado, contemplando y valorando no solo el diagnóstico, las secuelas psicofísicas del paciente que le sufre, también su edad, sexo, situación económica, lugar de residencia, habitabilidad de la vivienda, que pueden influir de manera directa en su rehabilitación y en su proceso de recuperación

\subsubsection{Sexo y edad}

El sexo condiciona determinados parámetros como por ejemplo la esperanza de vida, donde las mujeres tienen una mayor esperanza de vida que los hombres ${ }^{75}$. El riesgo de sufrir un accidente cerebrovascular entre los hombres es 1,25 veces el de las mujeres. La esperanza de vida de los hombres no es tan alta como el de las mujeres, por lo que los hombres son usualmente más jóvenes cuando sufren un accidente cerebrovascular y por 
tanto, tienen una tasa de supervivencia más elevada que las mujeres ${ }^{76} 77$. La evidencia científica señala que la aparición de ictus es más frecuente a partir de los 55 años $^{78}$. Su incidencia aumenta con cada década de la vida en ambos sexos, y es mayor en los varones de 45-84 años y en las mujeres de 85-94 años (esperanza de vida mayor en el grupo femenino una vez superados los 85 años $)^{79}$. La edad media con que acontece el primer ictus en los varones es de 69,8 años $(60,8-75,3)$, mientras que en las mujeres es de 74,8 años $(66,6-78,0)^{80}$.

Aproximadamente las tres cuartas partes de los ictus afectan a pacientes mayores de 65 años y debido a las previsiones de población en las que España será en el año 2050 de las poblaciones más envejecidas del mundo, se prevé un incremento de la incidencia de esta patología en los próximos años ${ }^{76}$. En relación con la recuperación funcional se evidenció en varios estudios la influencia negativa de la edad en la misma, aquellos con mayor deterioro de la capacidad funcional $(79,84 \text { años })^{81}$ coincide con los estudios de SantosIasaosa $^{82}$ y de Forelli ${ }^{83}$ que registraron medias superiores a los 70 años para los pacientes con una repercusión funcional diferente frente a una mejor evolución (64,15 años). A la luz de estos datos, existe un amplio margen de recuperación entre los 55 años (mayor frecuencia de aparición del ictus) hasta los 79 años, edad que se evidencio hay un mayor deterioro de la capacidad funcional. Sin embargo, personas entre 55-79 años son privadas de rehabilitación integral, ciertos tratamientos rehabilitadores públicos establecen la edad como criterio de exclusión para su acceso (55 años y 60 años), sin analizar otra serie de parámetros.

En relación con el sexo la evidencia científica muestra que las diferencias de sexo en torno al ictus se reflejan en numerosos estudios que plasman desigualdades en la incidencia, la prevalencia, los factores de riesgo, la presentación clínica, la demora prehospitalaria, el comportamiento del sistema sanitario y la comorbilidad de la enfermedad $^{84}$ se considera este factor también puede influir en el tratamiento rehabilitador. En España, los análisis realizados han detectado que las mujeres se enfrentan a una doble carga laboral, dentro y fuera de casa ${ }^{85}$ situación que puede repercutir en poder acceder y realizar una rehabilitación diaria y frecuente o en el tipo de rehabilitación que se ven obligadas a aceptar. Investigaciones muestran diferencias entorno al destino al alta hospitalaria de varones y mujeres que sufren ictus, en el caso de los varones al alta hospitalaria regresaron en mayor medida al domicilio, mientras que las mujeres precisaron más asistencia domiciliaria y fueron más institucionalizadas tras el alta $(p<0,005)^{84}$. El estudio publicado por Kapral et al. (2005) también señala que las 
mujeres son más propensas que los hombres a ser institucionalizados tras el alta del hospital ${ }^{86}$. Estos datos pueden tener también relación con la edad con la que sufren el ictus (mayor edad que el varón), las mujeres de edad avanzada sufren ictus más graves, aunque con más posibilidades de sobrevivir a un primer episodio que los hombres ${ }^{87}$. Una supervivencia, que según confirman varios estudios las mujeres sufren más complicaciones, y registran además estancias hospitalarias más prolongadas ${ }^{88} 8990$ y peor pronóstico. Estas estancias hospitalarias más prolongadas en las mujeres, también pueden tener relación con su mayor tasa de institucionalización, junto con la mayor edad y dependencia, que dificulta tratamientos rehabilitadores ambulatorios que conllevan desplazamientos continuos.

Se afirma en varios estudios que cada día de retraso en el tratamiento neurorrehabilitador, empeora el pronóstico funcional de los pacientes en el alta, demora a su vez relacionada con tasas de institucionalización más elevadas ${ }^{91}{ }^{92}$. Conocemos de la existencia de centros rehabilitadores, donde el criterio de la edad, es motivo de exclusión ${ }^{93}$, como el Ceadac y Guttman. Este criterio repercute en su recuperación funcional y puede estar relacionado con prolongación de estancias innecesarias.

\subsubsection{Estado civil o tener pareja}

El tener pareja se asocia a determinados estados emocionales y a percepción de sentimientos de soledad, afecto, compañía, apoyo, influye sobre la salud emocional y mental de las personas ${ }^{94}$, por tanto, en la predisposición de colaboración del paciente en su propio proceso de rehabilitación. La pareja tiene importancia de cara al apoyo emocional y material por la necesidad del paciente de una mayor atención afectiva y/o ayuda física. En este sentido, por ejemplo, existen investigaciones que indican que durante una enfermedad es más probable que la pareja proporcione apoyo material al paciente, ya que éste puede estar experimentando algún tipo de incapacidad funcional ${ }^{95}$.

- El matrimonio o vivir en pareja, parece aportar una estructura protectora contra la enfermedad $^{96}$. En esta relación de convivencia estable se produce un proceso en el cual la pareja se modifica y evoluciona continuamente, proporcionándose de forma mutua apoyo emocional, protección, seguridad y apoyo entre los 2 miembros ${ }^{97}$. La convivencia con la pareja es, de gran relevancia, ya que las diferencias en la salud no vienen dadas exclusivamente por el estado civil sino por la interacción entre ambas variables. Se da un efecto beneficioso de las uniones y la mejoría del bienestar físico y emocional tras la transición a la convivencia con una nueva pareja, frente a las 
personas que permanecen $\sin _{\text {esta }}{ }^{9899100}$. La convivencia en pareja podría representar un antídoto contra la enfermedad, al igual que otros tipos de relaciones de sociabilidad $^{101}$. Casado o vivir en pareja y con hijos puede influir en el acceso al tratamiento rehabilitador en dos direcciones, por un lado, tiene apoyo familiar; pero por otro, tiene responsabilidades familiares. Estas situaciones pueden ser un apoyo o una dificultad en el tratamiento rehabilitador. Teniendo en cuenta los resultados presentados en diferentes trabajos para España ${ }^{102} 103$ el hecho de que las personas casadas (o que viven en pareja) estén institucionalizadas es menos probable que para las personas viudas, puesto que el cónyuge se encarga de prestar el apoyo informal necesario. La edad del cónyuge se ha asociado a las mejoras de supervivencia, tener un cónyuge más joven es beneficioso para los hombres, pero perjudicial para las mujeres, mientras que tener un cónyuge mayor es perjudicial para ${ }^{104}$ ambos. Entre los efectos positivos se encuentran las mejoras psicológicas de tener una pareja más joven $^{104}$.

- En los casos de personas viudas, la viudedad supone un impacto emocional, económico y relacional. El vacío que origina el suceso tanto para los hombres como para las mujeres difícilmente es compensado por una figura similiar ${ }^{105}$. La pérdida de la compañía y la intimidad que procuraba el cónyuge es percibida por la práctica totalidad de mayores como una contrariedad de naturaleza irreparable ${ }^{105}$. Dependiendo del tiempo que ha transcurrido del suceso de fallecimiento de su cónyuge, pueden presentar sentimientos encontrados. Por un lado, la pérdida de ilusión por vivir que repercute en la recuperación y la ausencia de su cónyuge que le puede proveer de cuidados. Por otro, la disposición de tiempo y ausencia de cargas familiares, le permite disponer de tiempo para asumir un tratamiento rehabilitador intenso.

- Por el contrario, en el caso de pacientes soltero/a y/o sin cargas familiares que viven solos/as, la disponibilidad de tiempo para dedicar al proceso de rehabilitación es mayor, las responsabilidades familiares pueden ser menores. Sin embargo, el vivir solo/a y tener una baja frecuencia de actividades sociales, en estudios realizados se observó entre los factores que se asocian con la depresión post-ictus (DPI) ${ }^{95}$ lo que perjudica en el acceso al tratamiento rehabilitador y el buen desarrollo del mismo. Otros estudios revelan también cómo vivir solo/a aumenta la probabilidad de entrar en una institución ${ }^{106}$.

- Personas separadas o divorciadas: La ruptura de la unión conlleva efectos 
emocionales relacionados con el sentimiento de pérdida y de percepción de fracaso. Una experiencia que se desvela angustiosa aun en sociedades con tasas de divorcio elevadas ${ }^{107} 108$. Puede desencadenar además un aumento de la vulnerabilidad, puesto que constituye una transición vital que a menudo provoca aislamiento y sensación de quiebra del grupo de pertenencia ${ }^{108}$. En los casos de ruptura conflictiva, la duración del proceso puede dilatarse en el tiempo. De hecho, el divorcio no siempre implica el final del conflicto de la pareja, sobre todo cuando existen hijos comunes o bienes materiales compartidos ${ }^{109}$. El divorcio puede llegar a lesionar la salud mental ${ }^{110} 111$. Kalmijn (2010) utiliza un análisis multinivel que incluye 38 países desarrollados y aporta evidencias de que las personas divorciadas se encuentran en niveles de bienestar más bajos que las casadas, si bien las diferencias entre países son muy notorias $^{112}$.

En definitiva, el matrimonio proporciona estabilidad social y emocional, mientras que el divorcio, la separación, la viudedad y el estar sin pareja la socavan99 113114115.

Por tanto, estas situaciones pueden influir en el acceso a un tratamiento rehabilitador y la modalidad de tratamiento que se precisa. Es decir, contar con apoyo en el ámbito familiar puede posibilitar que el paciente permanezca en su domicilio y el tratamiento rehabilitador no requiera institucionalización.

\subsubsection{Unidad de convivencia}

Se entiende como unidad de convivencia ${ }^{116}$ la conformada por las personas que residen en una misma vivienda, independientemente de si existe parentesco de cualquier tipo entre ellas o no, o de si conformaran una familia en un momento futuro. La vivienda es el espacio social donde se desarrolla la vida privada, independientemente del número de personas que lo ocupen, o del momento del ciclo vital en que se encuentren, pudiendo estar ocupada por hogares unipersonales o pluripersonales, con o sin núcleo, y con otras personas (emparentadas o no) viviendo con ellos ${ }^{116}$.

Es decir, el conjunto de personas que viven habitualmente bajo el mismo techo, y que pueden estar ligadas entre ellas por lazos de parentesco, afinidad o amistad.

El efecto protector para la salud de la convivencia en pareja es bien conocido ${ }^{117}{ }^{118}$, el estado civil que revela peores riesgos para la salud es la de las personas separadas y divorciadas que no conviven en pareja ${ }^{119}$. Esta afirmación es válida, tanto para hombres como para mujeres, si atendemos a los riesgos de sufrir depresión crónica, mientras que en cuanto a la ansiedad crónica a ellos no les parece afectar la situación de convivencia y 
de estado civil ${ }^{94}$.

En general vivir con alguien, independientemente del estado civil y la relación de parentesco, se ha visto que tiene relación positiva con las conductas de adherencia ${ }^{120} 121$. La ventaja para la salud de las personas que viven en pareja se ha explicado ampliamente por un conjunto de beneficios relacionados con la convivencia: en primer lugar, el hecho de estar sometido a un control social, especialmente por parte de la pareja. Especialmente los hombres que viven en pareja pueden verse más animados a abandonar los comportamientos de riesgo que los solteros ${ }^{118}$. En segundo lugar, la combinación de las redes sociales de ambos miembros de la pareja crea una fuente más amplia de posibles recursos para reducir el impacto de los acontecimientos que pueden afectar negativamente a la salud ${ }^{122}$.

Las parejas también influyen en el estilo de vida del otro durante su relación (por ejemplo, es más difícil dejar de fumar si la pareja también fuma) y el parecido de la pareja en cuanto a la salud aumenta con la duración de la relación ${ }^{123}$. Cuando el marido tiene mala salud, es probable que la salud de la mujer se deteriore debido al aumento de su carga de trabajo doméstico ${ }^{124}$. Por el contrario, la carga de trabajo adicional es menor para los maridos cuando sus esposas tienen mala salud, ya que ésta suele compartirse con otros familiares, principalmente femeninos, sobre todo hijas o nueras ${ }^{125}$.

\subsubsection{Lugar de residencia}

El concepto de desigualdad en salud alude al impacto que tienen, sobre la distribución de la salud y la enfermedad en la población, los determinantes sociales como: la riqueza, la educación, la ocupación, el grupo racial o étnico, la residencia urbana o rural y las condiciones de habitabilidad o equipamientos del lugar en el que se vive o trabaja ${ }^{126}$. El lugar donde se reside por tanto afecta nuestra salud.

La dispersión geográfica se erige como una barrera para el tratamiento rehabilitador: ciertos tratamientos no son accesibles ni están disponibles en todas las localidades dónde viven las personas que sufren ictus.

El lugar de residencia influye de forma importante en la equidad sanitaria, influye directamente en la accesibilidad al tratamiento rehabilitador, no en todas las localidades existen los mismos recursos y apoyos. Además, no siempre existe el recurso que precisa ese paciente concreto, ni el procedimiento de acceso es igual de sencillo y posible para todos los pacientes: ausencia de medios de transporte públicos, limitaciones horarias en el acceso a las líneas regulares de transporte, carreteras sinuosas y complicadas, distancias 
geográficas de más de una hora.

En el caso de Castilla y León, su Sistema de Salud está constituido y delimitado en su territorio por demarcaciones denominadas Áreas de Salud ${ }^{127}$. Cada Área de Salud tiene al menos 1 hospital, en el caso de esta investigación el Área de Salud Este de Castilla y León, que cuenta con dos Hospitales. El estudio se centra en el Hospital Clínico Universitario de Valladolid la distancia geográfica máxima desde los pueblos que abarca hasta el Hospital para recibir tratamiento rehabilitador, supera en muchos casos los 60 kilómetros.

Aunque la cobertura sanitaria regional es total, existen agravios en el acceso de los pacientes a cada una de ellas, destacando: limitación funcional del paciente, la distancia geográfica, accesibilidad al transporte y/o las barreras arquitectónicas, entre otras. La rehabilitación motora en el domicilio está condicionada no solo por el diagnóstico sino por el lugar de residencia, por ejemplo, el Hospital Clínico Universitario de Valladolid solo cubre la ciudad de Valladolid, quedando desprotegidos de este tratamiento la zona rural del Área de salud Este.

\subsubsection{Vivienda}

Es fundamental que la vivienda cumpla unos requisitos de accesibilidad, movilidad y seguridad para que la persona que sufra ictus pueda ver cubierta sus necesidades reales y la permanencia en la misma mientras recibe el tratamiento rehabilitador sin que suponga un peligro para su salud. En ocasiones en la vivienda existen barreras físicas que dificultan la permanencia y deambulación en la misma. Lograr que se encuentren lo más cómodos posibles ayudas a mitigar su malestar, tristeza o agitación aumentando la sensación de seguridad y confianza y la posibilidad de acceder a un tratamiento rehabilitador permaneciendo en su entorno. Dificultades que puede presentar:

- Barreras arquitectónicas:

- No dispone de accesos habilitados para su movilidad y deambulación (escalera/sin ascensor para acceso vivienda o planta superior), puertas estrechas que impiden entrar las sillas de ruedas.

- Bañera: La persona presenta dificultades para caminar o se desplaza en silla de ruedas, con imposibilidad de entrar en una bañera, por el desnivel que presenta.

- Obstáculos de habitabilidad:

- La vivienda tiene muebles que tengan aristas peligrosas, mesitas de cristal, balancines o sillas poco estables o plegables. 
- Alfombras por las habitaciones.

Un entorno físico, emocional y social adaptado a ellos favorece su rendimiento diario y el de las personas cuidadoras. Es fundamental que el ambiente se adapte a las necesidades y capacidades que presentan provocadas por el ictus. El entorno debe ser sencillo, estable y seguro para protegerlos, facilitarles su autonomía y deambulación, hacer más agradable su vida y la de sus personas cuidadoras.

Es necesario que cumpla estas condiciones para posibilitar el acceso a un tratamiento rehabilitador permaneciendo en su domicilio y para que el tratamiento rehabilitador tenga eficacia. Al contrario, puede perjudicar en el proceso rehabilitador del paciente, obligando a esfuerzos desmedidos y que pueden dañar su estado de salud, exponiéndole a caídas, riesgos en el domicilio que perjudican y retrasan su recuperación funcional.

El régimen de tenencia es un segundo factor que puede influir en el acceso y tipo de tratamiento rehabilitador por los siguientes motivos:

- Que el paciente tenga una vivienda en su propiedad pagada, y que está vivienda no cumpla condiciones propicias/idóneas de habitabilidad o deambulación por la misma, posibilita alquilarla o venderla si es necesario para asumir una vivienda que este próxima al tratamiento rehabilitador (Ejemplo: Irse en alquiler a Valladolid si vive en zona rural para acudir diariamente al Hospital) o permita pagar este si no está siendo cubierto por el Sistema de Salud. Además, el tener la propiedad pagada, posibilita solvencia económica a la persona, que no está haciendo un desembolso si estuviese pagando un alquiler.

- Vivienda en alquiler, que presenta barreras que impiden su permanencia en la misma. Es posible buscar otra vivienda en alquiler próxima al tratamiento rehabilitador y garante de respuesta a sus necesidades básicas. Además, si la vivienda si cumpliese condiciones de habitabilidad, existe un gasto económico mensual en la persona que resta de su solvencia económica para pago de ciertos tratamientos rehabilitadores no cubiertos por los Sistemas Públicos.

- Cedida al uso sin pago: Si la vivienda cumple condiciones de habitabilidad y no llevan pago mensual, el paciente tiene menos gastos y puede disponer según su situación económica, de dinero para tratamiento rehabilitador. Cedida al uso pero no cumple condiciones de habitabilidad, según su situación económica podrá buscar o no otra vivienda en alquiler, un gasto económico en otra vivienda le supone menos recursos económicos disponibles para tratamiento rehabilitador. 


\subsubsection{Economía}

La situación económica es un determinante social de la salud, que se considera puede influir en el acceso al tratamiento rehabilitador del ictus y al pronóstico funcional del/la paciente, especialmente siendo conocedores del coste de este tipo de tratamientos y la falta de cobertura pública por el Sistema Público de Salud. Además, las necesidades que se suscitan alrededor del daño temporal que puede producir el ictus y que contribuyen a su proceso rehabilitador llevan un coste: adaptabilidad vivienda, cuidador familiar, transporte para desplazamiento, entre otros. Personas supervivientes a un ictus que presentaron la falta de autonomía se asoció con la presentación de mayores dificultades económicas ${ }^{128}$.

El coste económico influye en el acceso a la rehabilitación integral y su pronóstico funcional por varios motivos, entre ellos:

- El pago de tratamientos rehabilitadores no financiados por el sistema público de salud (no neuropsicología, no logopedia concertada en todos los hospitales, no rehabilitación visual, entre otros).

- El pago del coste del cuidado informal que precisan pacientes con ictus al alta hospitalaria y durante su periodo de rehabilitación integral.

El coste del cuidado informal de pacientes con ictus en la población general, realizado con una aproximación bottom-up mediante un cuestionario específico de recogida de actividades diarias, es de 21.551,28€/año, con un rango que varía, según el análisis de sensibilidad, entre $6.490,80$ y $31.436,72 € /$ año $^{129}$. El 40\% de los pacientes con una enfermedad cerebrovascular no institucionalizados precisan cuidados informales, con una media de 2 cuidadores por paciente y 5,6 h/día de cuidados. Según aumenta el grado de dependencia, el número de horas de cuidado y los costes son mayores. Así, el coste del cuidado informal aumenta desde los 10.442,9 €/año en los pacientes dependientes para las AIVD, hasta los 24.865,2€/año en los dependientes para las ABVD ${ }^{129}$.

Destacar dentro de la atención a las necesidades básicas de la vida diaria, que el riesgo de desnutrición es frecuente a corto plazo en pacientes con ictus y una desnutrición se asocia con una peor situación funcional, sin embargo, el mejor estado nutricional conlleva una mayor independencia funcional, mejor calidad de vida auto percibida y menor comorbilid $^{130}$. Hay evidencia que entre los factores de este riesgo de desnutrición se encuentra un menor nivel económico ${ }^{130}$. 


\subsection{Factores sociofamiliares}

La familia es en muchas ocasiones la fuente principal de apoyo con que cuenta el enfermo para afrontar con éxito los problemas a que da lugar la enfermedad. La familia cumple una serie de funciones como ${ }^{131}$ escenario de preparación dónde se aprende a afrontar retos, así como, asumir responsabilidades y compromisos; aporta un clima de afecto y apoyo.

En el tratamiento rehabilitador de un paciente con ictus la influencia familiar es un factor relevante. Las limitaciones tanto físicas como psíquicas que pueden surgir ante la aparición de un ictus, hace que cobre todavía mayor importancia el papel de la familia en el acceso, mantenimiento y apoyo al tratamiento rehabilitador del paciente, formando parte de ese proceso rehabilitador.

Tradicionalmente, la familia ha jugado un papel muy importante en la protección de sus miembros frente a la adversidad. Comparte junto con el enfermo, el medio ambiente tanto físico como social. Influye en el equilibrio emocional, puede actuar como génesis en problemas de salud, la enfermedad de un miembro afecta al grupo familiar. La familia es la principal cuidadora, las disfunciones, la desestructuración, las creencias, actitudes y comportamiento influyen en la recuperación y seguimiento del paciente ${ }^{131}$.

Por tanto, la familia tiene un peso significativo en el acceso al tratamiento rehabilitador, pero tener familia no garantiza tener apoyo o cuidador familiar, o que éste sea el adecuado. Es necesario discernir estos conceptos e identificarles para posicionarnos en el apoyo real familiar frente al acceso y tratamiento rehabilitador integral del paciente con ictus: a) Apoyo familiar: Se aplica la escala Apgar Familiar ${ }^{132}$ para comprobar si existe apoyo familiar y, b) Cuidador familiar, si existe cuidador familiar o va a existir después del ictus.

\subsubsection{Apoyo familiar}

En el ajuste a la enfermedad va a influir, además de características propias de la enfermedad y del propio paciente, el apoyo que recibe el paciente de su pareja dado su papel fundamental como principal fuente de apoyo ${ }^{133}$. En una investigación se evaluó ${ }^{134}$ el papel del apoyo en la experiencia de dolor crónico y obtuvo que la variable de apoyo de la red familiar se constituyó como predictiva de la ansiedad y depresión, así como del dolor en las enfermedades crónicas estudiadas.

En un episodio sobrevenido, como el ictus, es probable que al no existir dependencia funcional previa no hubiese cuidador familiar. Por tanto, convertirse en cuidadores puede 
suceder de forma inesperada o poco a poco, depende de cómo nuestro familiar al que se va a cuidar entra en la situación de dependencia (en el caso del ictus es de manera repentina). Esto conlleva que no estén adecuadamente preparados para ello. Por ello, es preciso conocer, la existencia o no de cuidador, si existe, cuál es la relación con el paciente o la de quién va a empezar a asumirlo, su experiencia, necesidades que presenta. La existencia de cuidador familiar es un condicionante en el acceso al tratamiento rehabilitador, puesto que las personas que desean seguir en sus domicilios y disponen de tratamiento rehabilitador ambulatorio pueden carecer de este apoyo continuo para dar atención a las ABVD, o quién lo asume carece de habilidades y conocimientos, o su estado de salud impide que las realice correctamente. Por tanto, no se valora el hecho de la existencia de un cuidador familiar, sino la importancia del saber cuidar. Ser el cuidador de la pareja es un conocido factor de riesgo para la salud física y mental ${ }^{135}$, una tarea que las mujeres cumplen con más frecuencia que los hombres debido a su mayor esperanza de vida y a los roles tradicionales de las normas de género ${ }^{136}$.

Se debe tener presente, que actualmente, la pérdida de vínculos familiares, modificaciones en la estructura familiar, necesidad de un alto nivel adquisitivo, el desarrollo a pasos agigantados de una sociedad de consumo y la crisis del Estado de Bienestar, han reducido la capacidad de respuesta de la familia ${ }^{131}$. Por ello, es preciso abordar otro tipo de apoyos, como el apoyo social.

\subsubsection{Apoyo social}

En diversos estudios epidemiológicos se muestra claramente un vínculo entre apoyo social y salud, donde el apoyo social se define como integración social o asentamiento dentro de una estructura social, de tal manera que sean posibles los contactos frecuentes con los otros ${ }^{131}$. El apoyo social es también una variable prometedora para predecir el resultado después del ictus. Una revisión reciente llegó a la conclusión de que los factores sociales se asociaron consistentemente con la calidad de vida postictus ${ }^{138}$.

La red de apoyo social de la persona puede influir en el mantenimiento de conductas deseables de salud, en el seguimiento de prescripciones médicas (dieta, medicación, ejercicio otros cambios en el estilo de vida) y en el cumplimiento de programas de rehabilitación, lo que nos estaría indicando la importancia tanto de las fuentes de apoyo como del tipo de apoyo que estas proporcionen de cara al cumplimiento de las recomendaciones pautadas ${ }^{139} 140$. 
Opuse propone una relación de nueve fuentes de apoyo social: cónyuge o compañero/a, otros familiares, amigos, vecinos, jefes o supervisores, compañeros de trabajo, personas de servicio o cuidadores, grupos de auto ayuda y profesionales de la salud o servicios sociales $^{141}$. Otras clasificaciones proponen la división de las redes de apoyo social en naturales (familia, amigos, compañeros de trabajo) y organizadas (grupos de ayuda mutua o asociaciones, voluntariado institucional, ayuda profesional). También se han clasificado en redes informales, (aquellas más cercanas al individuo siendo la principal, la familia) y en redes formales (formadas por aquellos individuos pertenecientes a la red de cuidadores de servicios sanitarios y sociales) ${ }^{141}$. Por tanto, en el apoyo social no solo se incluyen familiares y amigos, también forman parte de la red los profesionales socio sanitarios que están en contacto con el paciente y la familia. Juegan un papel clave en la adherencia al tratamiento, en la adaptación del binomio persona enferma -persona cuidadora a los recursos disponibles. De tal modo que un apoyo social inadecuado también podría llevar al incumplimiento terapéutico ${ }^{142} 143$. En nuestra investigación en la distinción/separación entre apoyo familiar y social (aunque hemos visto que hay autores que entienden el apoyo familiar como social), siguiendo el criterio de estudios que muestran aportaciones sobre la distinción de los efectos del apoyo familiar y social en la salud de las personas. En relación con la adherencia al ejercicio físico, se han encontrado existen diferencias significativas en función del apoyo social de los amigos ${ }^{144}$. En este caso, el apoyo social de los amigos ejerce mayor influencia en la realización del ejercicio físico que el apoyo familiar. El apoyo familiar, en cambio, será muy importante para el cumplimiento de la toma y administración de medicamentos ${ }^{144} 145$. En relación con el sexo, los hombres han contado con mayor apoyo social debido a su integración laboral, y las mujeres en cambio tradicionalmente han recibido más apoyo a nivel familiar y comunitario ${ }^{146}$.

Es indudable que la red de apoyo social cobra un papel muy relevante. Se considera puede influir en el acceso al tratamiento rehabilitador y en el buen desarrollo/ adherencia al mismo. Por ello, en lo que respecta a este punto, en nuestra investigación hemos abordado y analizado como apoyo social: la intervención social anterior al episodio con ictus con la trabajadora social; el reconocimiento de apoyo social formal por la Ley de promoción de la autonomía personal y atención a personas dependientes ${ }^{147}$, antes del ictus; y, si tienen apoyo de vecinos, voluntariado, asociaciones, entre otros.

Actualmente las investigaciones realizadas se centran y ofrecen datos en el mejoramiento de funciones y estructuras corporales, pero se necesita profundizar en el impacto de la rehabilitación integral en la vida de las personas, a nivel socio-profesional. 


\section{PAPEL DEL TRABAJO SOCIAL NEUROLÓGICO EN HCUV EN EL ACCESO A LA RHB INTEGRAL POST-ICTUS Y RECUPERACIÓN DEL PACIENTE}

\subsection{Origen}

El trabajo social neurológico en el Hospital Clínico Universitario de Valladolid (HCUV) se inicia en 2015, con la incorporación al Servicio Médico de Neurología, de la trabajadora social autora de esta tesis. El servicio de neurología nunca había contado antes con una trabajadora social sanitaria dedicada tan exclusivamente a la atención social de los pacientes ingresados por enfermedades del sistema nervioso central y/o periférico, entre ellos el ictus. El conocimiento especializado en este campo es fundamental, si desconocemos los problemas que puede provocar en el paciente una lesión cerebral, no podremos evaluar con precisión la comprensión del riesgo por su parte. Una evaluación especializada aporta una reducción del riesgo para los pacientes, que no se realiza en muchas ocasiones porque la formación en trabajo social no incluye en España un conocimiento especializado en este campo. Esta evaluación permite distinguir entre la capacidad intelectual del paciente para describir los riesgos a los que se enfrenta y su capacidad funcional para evitarles ${ }^{148}$. Las evaluaciones eficaces y precisas después de una lesión cerebral son una tarea cualificada y compleja que requiere conocimientos especializados ${ }^{148}$ y la formación universitaria en trabajo social es generalista

Por ello, la trabajadora social neurológica del HCUV, inicia el camino hacia la especialización en este campo con formación clínica y la elaboración y publicación de sistematizaciones y protocolos de intervención profesional. En el año 2016 se incorpora como miembro del equipo al programa de sesiones clínicas del Servicio Médico de Neurología. Durante estos años, el Trabajo Social Neurológico, ha ido afinando el diseño de una intervención social específica con la persona y familia que refleja el significado e implicaciones de la enfermedad que padecen.

El objetivo central de su intervención profesional, es lograr la recuperación máxima del paciente potencialmente recuperable y, en los casos en que no resultara posible restaurar todas las áreas funcionales afectadas, contribuir a su plena inclusión y participación en la sociedad. Es decir, garantizar al alta hospitalaria la provisión de recursos indispensables que aseguren la calidad de vida y el bienestar del paciente en las mejores condiciones posibles, respetando tanto los valores individuales de la persona como los Derechos Humanos. 


\subsection{Perfil del trabajo social neurológico (TSN) en el HCUV}

El desarrollo de sus funciones y una intervención social garante de la atención socio sanitaria que precisa el paciente y su familia, exige que el profesional del servicio de neurología tenga conocimientos específicos acreditados, entre otros: conocer con precisión la terminología clínica, las pruebas diagnósticas, los factores de riesgo, la estructura y funcionamiento de un servicio clínico de neurología, conocimiento y entendimiento de los distintos profesionales que lo conforman, dominio de protocolos y guías de intervención sobre las diferentes patologías, entre otros ${ }^{48}$.

Además, debe tener competencias concretas en técnicas propias y especificas del trabajo social en el área de neurología (uso de escalas de valoración, por ejemplo) y fundamentalmente participación en grupos científicos, publicaciones e investigaciones que contribuyan a acrecentar el conocimiento científico basado en la evidencia sobre todos los aspectos relacionados con la atención social y recuperación integral de pacientes en esta especialidad ${ }^{48}$. La comprensión de los diferentes factores de riesgo modificables y no modificables que influyen en la aparición y tratamiento de las enfermedades neurológicas. La enfermedad cerebrovascular está asociada a factores de riesgo no modificables: principalmente a la edad, sexo, raza y antecedentes familiares. Estos factores son inevitables y es fundamental entenderlos para que las personas reciban el tratamiento y monitorización específicos, por ser sujetos de alto riesgo que pueden beneficiarse de controles más rigurosos ${ }^{149}$. Permitiendo la posibilidad de establecer actuaciones y medidas de control en el proceso rehabilitador.

La formación generalista es una barrera final que dificulta en el ejercicio de la profesión. Se requiere una especialización en el campo de intervención, es indudable, que una base teórica sólida te permite ser un profesional eficaz, ya que en el trabajo diario es más probable reconocer problemas o reacciones clave si tenemos una base de conocimientos a los que recurrir ${ }^{150}$.

Las habilidades propias de la trabajadora social reforzadas con una especialización en este campo hacen que las intervenciones generen mayor beneficio al paciente, al Servicio de Neurología y a la sociedad en general. 


\subsection{Proceso de intervención de Trabajo Social Neurológico en la Rehabilitación Integral post-ictus}

Es creciente el número de personas que viven con lesiones cerebrales adquiridas, que requieren ${ }^{145}$ atención médica inmediata y atención rehabilitadora y social a largo plazo. A pesar de sus necesidades sociales, se ha prestado una atención limitada a estas personas dentro de la literatura de trabajo social.

Algunos de los efectos secundarios comunes que presentan estos pacientes y que también requieren atención desde el trabajo social son: dificultades físicas y cognitivas, deterioro del funcionamiento ejecutivo, cambios en el comportamiento de una persona y cambios en la regulación emocional y la personalidad. Todos estos efectos secundarios tienen implicaciones significativas para todas las tareas de trabajo social, incluida la evaluación de las necesidades, la evaluación de la capacidad mental, la evaluación del riesgo y la protección $^{151}$. A pesar de ello, los equipos especializados de apoyo a las personas son prácticamente inauditos en el sector, y muchas personas tienen dificultades para obtener los servicios que necesitan ${ }^{152}$. Acceder al apoyo puede ser difícil porque las necesidades heterogéneas de estas personas no encajan fácilmente en las especialidades de trabajo social ${ }^{153}$. Las personas con ictus no salen del Hospital y regresan a sus domicilios con una imagen completa, clara y precisa de cómo los cambios causados por su lesión les afectarán. Se diseñó e implanto el proceso específico de intervención del trabajo social neurológico del HCUV con pacientes que sufren ictus y sus familias desde el ingreso hospitalario que continua después del alta hospitalaria, en un proceso de atención estructurado secuencialmente como se detalla a continuación ${ }^{48}$.

\subsubsection{Acogida}

El ingreso en el HCUV de un paciente por ictus es puesto en conocimiento de la trabajadora social neurológica. Los especialistas o enfermería realizan una interconsulta por el programa informático del HCUV, por vía telefónica o informan a la trabajadora social de neurología en las sesiones clínicas del Servicio Médico de Neurología. El proceso de atención social se inicia entre las 24-48 horas desde la comunicación a la trabajadora social neurológica. Se inicia esta fase con la visita en planta al paciente y/o localización de familiares o personas de su entorno. El proceso se adapta desde el inicio al daño y nivel de comunicación y entendimiento del paciente. La trabajadora social neurológica a través de la historia clínica y del Neurólogo/a o Especialista en Neurología analiza y averigua previamente a la visita dos aspectos claves y relevantes, su capacidad 
cognitiva y si padece trastornos de comprensión y comunicación (afasia, disartria) para encuadrar la entrevista a mantener en planta con el paciente. Esta primera visita se centra en que el/la paciente conozca a la trabajadora social de neurología, sus funciones y competencias, los motivos por los que recurrir a ella, modo de localización e intervención que va a realizar, en definitiva, conozca que es el profesional que le va a garantizar el acompañamiento social desde ese momento.

\subsubsection{Anamnesis social}

La trabajadora social neurológica inicia una recogida de datos de la situación clínica y sociofamiliar, para adentrarse en el conocimiento de la persona, su medio y su familia, a través de las diferentes herramientas de recopilación de información: fundamentalmente a través de la propia persona, su familia, el equipo de neurología y otras instituciones con las que guarda relación, entre otros. Posteriormente realiza la valoración del trabajo social neurológico considerando al paciente, la familia y la unidad de convivencia.

\section{Recopilación de datos sociales del paciente:}

En las entrevistas iniciales con el/la paciente desde su ingreso hospitalario, se trata de conocer las necesidades que presentaba previas al ictus, aquellas que van surgiendo y las que se prevé surgirán por las secuelas producidas por el ictus. Se realiza una valoración exhaustiva, basada en identificar y conocer los siguientes aspectos:

- La enfermedad acontecida: tipo de ictus, secuelas o déficits producidos, pronóstico (temporal o permanente), capacidad de conocimiento y comprensión, antecedentes de ictus de repetición, factores de riesgo modificables y no modificables presentes en el paciente, nivel de conocimiento que tiene sobre la enfermedad sobrevenida ${ }^{48}$;

- Características de su personalidad: capacidad de superación, afrontamiento, tolerancia a la frustración. Identificando aquellos factores personales que aumenten su vulnerabilidad, para intervenir sobre ellos y disminuirlos, también los factores protectores que incrementan respuestas resilientes en los pacientes;

- Situación socioeconómica: ingresos económicos del paciente y la unidad de convivencia, deudas, situación laboral (tipo de contrato que tiene y horario);

- Vivienda: tipo de vivienda en la que vive (chalé, piso, casa), barreras que dificultan su permanencia en la misma (sin ascensor, bañera, puertas estrechas), régimen de tenencia (alquiler, cedido uso, propiedad pagada, propiedad pagando hipoteca y las cantidades), accesibilidad a servicios de primera necesidad;

- Lugar de residencia:ZZona rural o urbana, distancia de los recursos básicos para cubrir 
sus necesidades básicas y distancia de los recursos que garantizan la rehabilitación que precisa, estado de las carreteras, vías de acceso, transportes públicos. Y fundamental conocer el momento o fase del ciclo vital en la que se encuentra el paciente cuando sufre el ictus: hijos pequeños, adolescentes, en proceso emancipación, con abuelos viviendo en el hogar, con padres o madres jubilados

La familia que puede ser una red de apoyo esencial, puede ser muy amplia y no pertenecer al núcleo de convivencia o la persona no vive con ningún familiar. Por tanto, la trabajadora social neurológica diferencia entre la unidad familiar y de convivencia y recoge información de ambas unidades.

Recopilación de datos sociales de la familia:

La trabajadora social de neurología del HCUV, para realizar una intervención personalizada y de calidad con la familia, realiza una valoración de la misma, que se centra en datos referentes a: la enfermedad acontecida, ecosistema de la familia, su estructura y funcionamiento, estilo de interacción situación socioeconómica, vivienda, lugar de residencia, problemas de salud en la familia y la figura del cuidador familiar si existe.

Recopilación de datos sociales de la unidad de convivencia:

La unidad de convivencia puede estar constituida por miembros de la familia, y está compuesta por otro tipo de personas: compañeros de piso, amigos, entre otros. Se recopila la siguiente información sobre los miembros de la unidad de convivencia, el reparto de las tareas de la vivienda, la situación sociolaboral de los miembros, la existencia de problemas de salud, si algún miembro de la unidad de convivencia desempeña el rol de cuidador. 
Tabla 2. Datos sociales comunes de la familia y de la unidad de convivencia:

\begin{tabular}{|c|c|c|}
\hline Aspectos & Unidad Convivencia & Unidad Familiar \\
\hline 1.Organización & \multicolumn{2}{|c|}{$\begin{array}{l}\text { Como se reparten las tareas internas del hogar y de la familia con } \\
\text { otros sistemas sociales }\end{array}$} \\
\hline 2.Cohesión & \multicolumn{2}{|c|}{$\begin{array}{l}\text { Está unida o dispersa la unidad ante los problemas que han ido } \\
\text { sufriendo }\end{array}$} \\
\hline 3.Conflictividad & \multicolumn{2}{|c|}{$\begin{array}{l}\text { Han tenido enfrentamientos en alguna ocasión, que motivos les } \\
\text { hacen enfrentarse, como abordan esos conflictos }\end{array}$} \\
\hline $\begin{array}{l}\text { 4.Expresividad } \\
\text { emocional }\end{array}$ & \multicolumn{2}{|c|}{$\begin{array}{l}\text { Expresan asertivamente lo que sienten, lo hacen de manera } \\
\text { frecuente o solo cuando están al límite }\end{array}$} \\
\hline 5.Adaptabilidad & \multicolumn{2}{|c|}{$\begin{array}{l}\text { Como han resuelto las adversidades, cuando se enfrentaron a } \\
\text { problemas quién asumió el liderazgo, quién se dejó llevar, como } \\
\text { se repartieron las responsabilidades, tipo de representaciones } \\
\text { dominantes en la unidad. }\end{array}$} \\
\hline $\begin{array}{l}\text { 6.Integración } \\
\text { emocional }\end{array}$ & \multicolumn{2}{|c|}{$\begin{array}{l}\text { Tienen capacidad de contención, quién consuela cuando hay un } \\
\text { problema, quién muestra más afecto, se enfrenta el sufrimiento, } \\
\text { hay alguien que sufra más, quien carga sus penas en el otro, hay } \\
\text { alguno que sea el portador del problema y otro va de victima }\end{array}$} \\
\hline $\begin{array}{l}\text { 7.Capacidad de } \\
\text { expresión } \\
\text { emocional. }\end{array}$ & \multicolumn{2}{|c|}{$\begin{array}{l}\text { Como manejan la expresividad emocional. Si expresan las } \\
\text { emociones, como las expresan, todos se dan cuenta de lo que } \\
\text { expresa cada uno, saben interpretarlo correctamente. }\end{array}$} \\
\hline $\begin{array}{l}\text { 8.Funciones } \\
\text { emocionales } \\
\text { que están más } \\
\text { afectadas }\end{array}$ & \multicolumn{2}{|c|}{$\begin{array}{l}\text { La situación problema: a qué y a quién está afectando, } \\
\text { indicadores de riesgo detectados. }\end{array}$} \\
\hline
\end{tabular}

Fuente: Elaboración propia.

Toda esta información es incorporada a la historia social del paciente y sistematizada para proceder con eficacia a establecer las fases siguientes.

\subsubsection{Diagnóstico-valoración de TSN}

En esta fase se definen y concretan los factores que inciden en la situación de necesidad/problema psicosocial del paciente y su entorno (fortalezas y amenazas) que después se adhieren al plan de rehabilitación integral multidisciplinar. Las herramientas de diagnóstico que aplica la trabajadora social de neurología del HCUV son ${ }^{48} 24$ : escala de Lawton y Brody ${ }^{154}$ para conocer la influencia de factores psicosociales en la aparición de problemas de salud, la escala Gijón ${ }^{155}$ para identificar el riesgo social que existe, la escala de Zarit ${ }^{156}$ para la detección de sobrecarga familiar, la escala Barthel ${ }^{157}$ para evaluar 
la valoración funcional de la persona, la entrevista psicosocial individual y familiar, la historia del vínculo y las evidencias documentales. Se recogen los códigos diagnósticos $\mathrm{Z}$ de la clasificación internacional CIE-10 $13{ }^{48}$.

La valoración social realizada por la trabajadora social de neurología se integra ${ }^{48}$ en la siguiente fase dentro de la valoración multidisciplinar alta hospitalaria. Esta valoración queda registrada en el programa informático Gacela.

\subsubsection{Valoración multidisciplinar}

En el HCUV se reúne con carácter semanal el equipo multidisciplinar que atiende al paciente con ictus (rehabilitador/a, enfermero/a, neurólogo/a, trabajadora social neurológica, logopeda, fisioterapeuta, entre otros) a través de una reunión semanal formal y reuniones informales si algún caso lo requiere antes de la siguiente reunión programada $^{93}$. En este espacio se abordan las complejidades de los problemas sociosanitarios que se vayan sucediendo. Se pretende minimizar los déficits, maximizar la función, prevenir las complicaciones específicas de cada paciente, logrando cuando es posible su mayor recuperación.

La trabajadora social neurológica forma parte del equipo sanitario de atención al paciente con ictus, lo que permite que el paciente desde su ingreso disponga de la atención social necesaria. Su intervención profesional se integra desde el inicio en las actuaciones conjuntas del equipo, lo que permite dar respuesta al conjunto de las áreas afectadas del paciente y su familia ${ }^{48}$.

En estas reuniones se establece previamente una valoración clínica que, junto con la valoración social, originan la propuesta inicial del Plan individualizado post-alta hospitalaria que necesita el paciente.

La decisión personal del paciente, junto con la disponibilidad real de recursos para recibir el tratamiento rehabilitador integral requerido, son los dos aspectos básicos que determinarán el Plan individualizado post alta hospitalaria que efectivamente reciba el paciente. Dicho en otras palabras: el tratamiento rehabilitador integral que el paciente necesita es lo que se incluye en la propuesta inicial de Plan individualizado. Pero esa propuesta inicial no será, en muchos casos, el Plan individualizado definitivo que finalmente reciba el paciente; ya que su implantación está condicionada por la disponibilidad del recurso, el acceso al mismo y el compromiso posible del paciente y su entorno familiar con dicho tratamiento. 
Tabla 3. Valoración clínica

\begin{tabular}{|c|c|}
\hline Aspectos & Objetivos/Motivos \\
\hline $\begin{array}{l}\text { 1-Intensidad de } \\
\text { deficiencias }\end{array}$ & $\begin{array}{l}\text { Conocer en qué medida se ha limitado o disminuido el } \\
\text { sistema funcional o mecanismo del cuerpo del paciente, } \\
\text { para qué actividades, con qué carácter (temporal o } \\
\text { permanente). }\end{array}$ \\
\hline 2-Edad & $\begin{array}{l}\text { Es un factor de riesgo no solo en el padecimiento de un } \\
\text { ictus influye también en su rehabilitación, frecuentemente } \\
\text { las personas de más edad tienen un deterioro funcional } \\
\text { previo. }\end{array}$ \\
\hline $\begin{array}{l}\text { 3-Depresión } \\
\text { postictal }\end{array}$ & $\begin{array}{l}\text { Identificar/Conocer: } \\
\text { - Si sufren o experimentan alteraciones del estado de ánimo } \\
\text { a raíz de padecer un ictus. } \\
\text { - Como lo manifiestan. }\end{array}$ \\
\hline 4-Función motora & $\begin{array}{l}\text { Identificar y tener en cuenta si presenta flacidez y } \\
\text { espasticidad intensas. Mantenidas son un factor de mal } \\
\text { pronóstico. }\end{array}$ \\
\hline 5-Lenguaje & $\begin{array}{l}\text { Identificar trastornos del lenguaje y conocer la gravedad } \\
\text { inicial. }\end{array}$ \\
\hline 6-Equilibrio & $\begin{array}{l}\text { Identificar la falta de control de tronco en sedestación. Este } \\
\text { constituye un factor de mal pronóstico. }\end{array}$ \\
\hline 7-Deambulación & $\begin{array}{l}\text { Identificar alteraciones en la deambulación. Teniendo } \\
\text { presente la alta probabilidad de recuperación en la marcha } \\
\text { normal está relacionada con déficit motor, afectación } \\
\text { sensitiva, visual y edad. }\end{array}$ \\
\hline $\begin{array}{l}\text { 8-Independencia } \\
\text { funcional }\end{array}$ & $\begin{array}{l}\text { Identificar a través de la aplicación del Barthel. El mal } \\
\text { pronóstico funcional se prevé cuando el índice Barthel } \\
\text { señala menos de } 20 \text { puntos, dependencia total }{ }^{157} \text {. }\end{array}$ \\
\hline $\begin{array}{l}\text { 9-Deterioro } \\
\text { cognitivo }\end{array}$ & $\begin{array}{l}\text { Identificar a través del test minimental }{ }^{158} \text {. Tiene mucha } \\
\text { relación con el acceso y seguimiento del tratamiento } \\
\text { rehabilitador. Tiene valor predictivo para el pronóstico, } \\
\text { muchos pacientes con deterioro cognitivo presentan } \\
\text { cuadros afásicos marcados, trastornos de función ejecutiva. }\end{array}$ \\
\hline $\begin{array}{l}\text { 10-Deficiencia } \\
\text { campo visual }\end{array}$ & $\begin{array}{l}\text { Es necesario identificarlas: } \\
\text { Las disminuciones o deficiencias visuales implican } \\
\text { aislamiento, dificultad de relacionarse, incapacidad de } \\
\text { visualizar la secuencia de ejercicios del plan de } \\
\text { rehabilitación establecido. }\end{array}$ \\
\hline 11-Tipo de ictus & Isquémico u hemorrágico \\
\hline $\begin{array}{l}\text { 12-Gravedad del } \\
\text { ictus }\end{array}$ & A través de la escala Nihss ${ }^{159}$ \\
\hline 13-Rankin & $\begin{array}{l}\text { El resultado funcional tras un ictus, se aplica la Escala } \\
\text { Rankin } \mathbf{1 6 0}\end{array}$ \\
\hline $\begin{array}{l}\text { 14-Tamaño y } \\
\text { localización del } \\
\text { infarto }\end{array}$ & $\begin{array}{l}\text { Se recogen estos aspectos fundamentales: } \\
\text {-Mayor volumen lesión: mayor gravedad } \\
\text {-Localización: se asocia a peor pronostico }\end{array}$ \\
\hline
\end{tabular}

Fuente: Elaboración propia. 
Una vez el paciente está estabilizado clínicamente y presenta una clara situación clínica, el equipo valora las áreas afectadas y la estimación pronostica.

La valoración temprana en una persona con $\mathrm{ACV}$ de su pronóstico de función a medio y largo plazo permite comunicarnos con el paciente y sus familiares en función de su capacidad de comunicación, identificar unos objetivos realistas de rehabilitación y planificar la derivación del enfermo más idónea al alta hospitalaria.

Los pacientes que sobreviven a un ictus pueden sufrir deterioro cognitivo, la evidencia sugiere que la información sobre lesiones cerebrales y su impacto cognitivo no se transmite adecuadamente a los trabajadores sociales ${ }^{161}$. En este espacio de encuentro entre los diferentes profesionales, se contribuye a paliar esta deficiencia y que la trabajadora social neurológica establezca su valoración correctamente. Ante un diagnóstico de deterioro cognitivo, la trabajadora social neurológica informa y orienta a la familia sobre el proceso de incapacitación. Si no existe familia es la propia trabajadora social neurológica quién inicia el procedimiento de incapacitación y petición de medidas cautelares para proteger la integridad del paciente al alta hospitalaria.

La valoración clínica determina el plan posthospitalización al alta. Si el paciente presenta una situación paliativa o una perdida funcional que conlleva una dependencia funcional crónica, la trabajadora social neurológica realiza la valoración social y establece el apoyo al alta en base a esta valoración clínica y las necesidades sociofamiliares del paciente.

La valoración clínica determina que el paciente es susceptible de tratamiento rehabilitador porque cumple los criterios de inclusión para adherirse al mismo: capacidad cognitiva preservada que le permite seguir las instrucciones rehabilitadoras, no tiene pérdida irrecuperable de función, no presenta una situación que requiere atención paliativa, se interviene en base a esta situación clínica.

La trabajadora social aporta al equipo multidisciplinar la valoración social realizada en la fase anterior. Esta valoración social permite obtener y organizar la información necesaria para decidir el tratamiento rehabilitador del paciente al alta hospitalaria.

Por tanto, se establece una valoración integral que describe la repercusión funcional del individuo para realizar actividades y desenvolverse en su entorno. Además, en un contexto donde el tratamiento rehabilitador integral está condicionado por la ausencia de tratamientos rehabilitadores públicos integrales, la trabajadora social expone las dificultades que pueden influir en su acceso y por tanto, en el pronóstico funcional. 
Tabla 4. Valoración trabajo social neurológico

\begin{tabular}{|c|c|}
\hline $\begin{array}{l}\text { 1-Apoyo social } \\
\text { (Aplicación cuestionario } \\
\text { Mos) })^{162}\end{array}$ & $\begin{array}{l}\text { Identificar si el paciente contaba con apoyo social } \\
\text { previo (Amigos, vecinos, entre otros) } \\
\text { Si cuenta con apoyo social por valoración de } \\
\text { reconocimiento dependencia } \\
\text { Si había iniciado previo al ingreso hospitalario } \\
\text { intervención social. }\end{array}$ \\
\hline $\begin{array}{l}\text { 2-Apoyo familiar } \\
\text { Clave en el proceso de } \\
\text { recuperación del paciente } \\
\text { (Aplicación Escala Apgar } \\
\text { Familiar) }\end{array}$ & $\begin{array}{l}\text { Identificar: } \\
\text {-Existencia de familia } \\
\text {-La implicación de la familia en la consecución de } \\
\text { los objetivos y en el programa de tratamiento } \\
\text { rehabilitador del ictus es fundamental } \\
\text {-Si las relaciones son sanas y positivas entre los } \\
\text { miembros. }\end{array}$ \\
\hline $\begin{array}{l}\text { 3-Lugar de residencia del } \\
\text { paciente } \\
\text { Imprescindible conocer para el } \\
\text { proceso rehabilitador: }\end{array}$ & $\begin{array}{l}\text { Identificar: } \\
\text {-Localidad en la que reside } \\
\text {-Disponibilidad de recursos en la misma } \\
\text {-Distancia de los recursos que precisa y de los } \\
\text { familiares. } \\
\text {-Estado de carreteras y medios de transporte } \\
\text { disponibles. }\end{array}$ \\
\hline $\begin{array}{l}\text { 4-Vivienda } \\
\text { Determinante para posibilitar } \\
\text { la permanencia de la persona } \\
\text { en su domicilio, }\end{array}$ & $\begin{array}{l}\text { Identificar: } \\
\text {-Condiciones habitabilidad de la vivienda } \\
\text {-Obstáculos o barreras que impiden la } \\
\text { deambulación (escaleras, no ascensor, no rampas) } \\
\text {-Régimen de tenencia }\end{array}$ \\
\hline $\begin{array}{l}\text { 5-Situación económica } \\
\text { Clave sino es posible acceder } \\
\text { públicamente a todo el } \\
\text { tratamiento que precisa. }\end{array}$ & $\begin{array}{l}\text { Identificar: } \\
\text {-Ingresos económicos de la persona y procedencia } \\
\text { (trabajo, prestaciones, jubilación) } \\
\text {-Deudas y gastos de primera necesidad. }\end{array}$ \\
\hline $\begin{array}{l}\text { 6.Personalidad del afectado } \\
\text { Aspecto clave en la capacidad } \\
\text { de adherencia y realización del } \\
\text { tratamiento rehabilitador }\end{array}$ & $\begin{array}{l}\text { Identificar: } \\
\text {-Problemas que ha tenido a lo largo de su vida y } \\
\text { como les ha afrontado } \\
\text {-Capacidad de superación y afrontamiento. }\end{array}$ \\
\hline $\begin{array}{l}\text { 7-Cuidador familiar } \\
\text { Fundamental en el proceso de } \\
\text { recuperación }\end{array}$ & $\begin{array}{l}\text { Identificar: } \\
\text {-Existencia de cuidador y qué relación tienen } \\
\text {-Tiempo y dedicación al cuidado } \\
\text {-Que conocimiento tiene del cuidado a ejercer. } \\
\text {-Es dependiente funcional o necesita atención. }\end{array}$ \\
\hline $\begin{array}{l}\text { 8-Situación laboral. } \\
\text { Aspecto fundamental en el } \\
\text { proceso rehabilitador puede } \\
\text { favorecer u obstaculizar en el } \\
\text { mismo }\end{array}$ & $\begin{array}{l}\text { Identificar: } \\
\text {-Trabajo que desempeña } \\
\text {-Tipo de contrato (fijo, temporal, negocio propio) } \\
\text {-Horario. }\end{array}$ \\
\hline $\begin{array}{l}\text { 9-Riesgo social del paciente } \\
\text { (Escala Gijón) }^{155}\end{array}$ & $\begin{array}{l}\text { Si existe posibilidad de sufrir daño derivado de } \\
\text { una causa social. } \\
\text { Si ese riesgo social perjudica en su estado de } \\
\text { salud- recuperación. }\end{array}$ \\
\hline
\end{tabular}

Fuente: Elaboración propia 


\subsubsection{Planificación del tratamiento rehabilitador al alta hospitalaria}

Al alta hospitalaria el paciente y familia se van a enfrentar con unos déficits y secuelas al mundo exterior. Es necesaria una adecuada planificación del recurso rehabilitador integral al alta para mantener y garantizar la continuidad de la atención socio sanitaria que precisa.

\section{A/. Propuesta multidisciplinar de tratamiento rehabilitador integral requerido al alta}

Las valoraciones del equipo multidisciplinar expuestas en la fase anterior, determinan el tratamiento rehabilitador integral que precisa al alta hospitalaria. En esta fase se establece la propuesta de tratamiento rehabilitador idónea para la mayor recuperación integral de este paciente ${ }^{93}$. La trabajadora social neurológica convoca al equipo y recoge en un documento el tratamiento rehabilitador integral propuesto, guardado en la historia clínica del paciente.

Tabla 5. Tratamiento rehabilitador que precisa al alta hospitalaria

\begin{tabular}{|l|l|}
\hline \multicolumn{1}{|c|}{ Componentes del plan } & \multicolumn{1}{c|}{ Paciente } \\
\hline $\begin{array}{l}\text { 1-Terapias rehabilitadoras que } \\
\text { precisa (6 dominios afectados) }\end{array}$ & $\begin{array}{l}\text { Áreas afectadas en la persona y } \\
\text { tratamiento que cubre cada área/dominio. }\end{array}$ \\
\hline $\begin{array}{l}\text { 2-Intensidad necesaria de cada } \\
\text { terapia. }\end{array}$ & $\begin{array}{l}\text { Tiempo de terapia que precisa en cada } \\
\text { dominio para su buena recuperación. }\end{array}$ \\
\hline $\begin{array}{l}\text { 3-Estimación-duración del } \\
\text { tratamiento rehabilitador. }\end{array}$ & $\begin{array}{l}\text { Cuánto tiempo de duración del programa } \\
\text { de rehabilitación. Se establece duración } \\
\text { del recurso y controles de seguimiento }\end{array}$ \\
\hline $\begin{array}{l}\text { 4-Tolerancia o capacidad de } \\
\text { resistencia del paciente al } \\
\text { tratamiento }\end{array}$ & $\begin{array}{l}\text { Nivel de tolerancia del paciente en el } \\
\text { mismo. Cualidades, conocimientos y } \\
\text { aptitudes que influyen en proceso } \\
\text { rehabilitador. }\end{array}$ \\
\hline $\begin{array}{l}\text { 5-Necesidades para la realización de } \\
\text { las ABVD }\end{array}$ & $\begin{array}{l}\text { Se plasma individualmente las tareas para } \\
\text { las actividades de la vida diaria que no } \\
\text { realiza el paciente a consecuencia de las } \\
\text { áreas afectadas }\end{array}$ \\
\hline 6-Decisión paciente & $\begin{array}{l}\text { El Paciente se le pregunta cuál es su } \\
\text { preferencia al alta hospitalaria (ir al } \\
\text { domicilio o no quiere volver al mismo) }\end{array}$ \\
\hline
\end{tabular}

Fuente: Elaboración propia 
La trabajadora social neurológica:

- Implica de manera activa al paciente y familiares en el tratamiento y en la toma de decisiones.

- Busca recursos que respondan al tratamiento prescrito por el equipo multidisciplinar ${ }^{57}$.

- Posibilita mientras el paciente lo necesite la continuidad del tratamiento rehabilitador.

- La intervención de esta profesional es clave para lograr que el tratamiento rehabilitador que se implante sea el propuesto por el equipo multidisciplinar o dada su imposibilidad, el tratamiento que cubra los mayores dominios afectados en el paciente.

\section{B/. Comunicación y potencial compromiso de paciente y familia en tratamiento} rehabilitador

El neurólogo/a o especialista en neurología y la trabajadora social comunican al paciente la valoración integral postalta y el plan rehabilitador integral propuesto inicialmente para lograr su máxima recuperación. Se dedica tiempo en esta fase a conseguir que el paciente y su familia comprendan los objetivos reales de la rehabilitación para evitar falsas esperanzas o expectativas inalcanzables ${ }^{163164165}$. La trabajadora social neurológica inicia junto al/la paciente, potenciando su autonomía, decisión y participación, el establecimiento del plan rehabilitador integral que recibirá al alta hospitalaria. En el transcurso de esta fase puede evidenciarse que el paciente no ha tomado conciencia de las secuelas producidas por el ictus, un problema muy característico que impide o dificulta su recuperación ${ }^{166}{ }^{167}$. La recuperación o mantenimiento de su salud, la superación de sus discapacidades conlleva necesariamente sea conocedor de su situación y su capacidad para utilizar los recursos del entorno físico y social ${ }^{164} 165$

Ante esta situación la competencia del trabajo social neurológico es clave y fundamental. Desempeña varias funciones:

- Realiza acciones orientadas a incrementar en los pacientes la comprensión del suceso acontecido,

- Potencia discurso motivador que contribuya a la adherencia del paciente al plan de rehabilitación integral. Destaca con sus verbalizaciones las fortalezas del paciente 
en su proceso rehabilitador.

Cuando el paciente es consciente del suceso acontecido y de sus limitaciones y necesidades al alta hospitalaria, tiene dos alternativas de decisión: desplazarse temporalmente a un centro de rehabilitación integral o retornar a su domicilio.

Desplazarse a centro de rehabilitación: Está decisión está condicionada por diferentes circunstancias: a) No puede acceder al centro que le ofrece el tratamiento rehabilitador integral público que precisa, criterios como la edad u otros, impiden su acceso; b) Puede recibir públicamente parte del tratamiento rehabilitador integral, cubriendo alguno de los dominios afectados (fisioterapia o logopedia) y otros dominios no.

Retornar al domicilio: El paciente manifiesta su deseo de volver al domicilio. $\mathrm{Su}$ situación clínica y social determina que existen dificultades para recibir desde su domicilio el tratamiento rehabilitador integral propuesto, por alguno de los siguientes motivos: a) El tratamiento rehabilitador público no está garantizada en su localidad de residencia (por ejemplo, la rehabilitación domiciliaria); b) El paciente necesita atención para las actividades básicas de la vida diaria. En este caso, si el paciente tiene familia, pero ésta no puede (o no quiere) colaborar, la TSN interviene con la familia para posibilitar e incentivar su colaboración cuando ello posible. Los motivos más frecuentes de esta situación son los siguientes: Insuficiencia para manejar la situación; la familia está en riesgo de exclusión; la persona con la que vive es dependiente; existen antecedentes de problemas emocionales y convivenciales; tienen horarios laborales incompatibles con el apoyo; viven lejos del lugar de residencia del paciente, entre otros. Ante estas situaciones, la trabajadora social neurológica orienta y asesora sobre apoyo informal en el domicilio que atienda sus ABVD. El establecimiento o contratación de este apoyo está condicionado por estas posibles circunstancias: a) el paciente tiene una situación económica que le permite contratar esos servicios; b) económicamente no puede pagar la atención que requiere su persona.

Ante este último supuesto, se interviene con la familia para promover su colaboración económica en la cobertura de la atención a sus necesidades personales y domésticas, no siempre tienen posibilidad.

C/. Búsqueda y selección de recursos potencialmente disponibles

La trabajadora social neurológica conoce los diferentes recursos tanto públicos, concertados como privados que dan respuesta a las áreas afectadas del paciente. Conoce 
su ámbito de actuación: nacional, autonómico, local; su ubicación, el sistema de acceso, objetivos, criterios de inclusión y exclusión, duración, normas de funcionamiento, tratamientos que ofrecen, profesionales que forman parte del equipo, su coste en el caso de los privados o los públicos que llevan copago.

Además, en el caso de los tratamientos públicos o concertados, conoce las plazas disponibles y la fecha aproximada de posible ingreso, algo fundamental para no retrasar el acceso al tratamiento rehabilitador y en el caso de recursos privados realiza los contactos pertinentes para conocer su disponibilidad.

Entre los recursos que gestiona la trabajadora social de neurología están los siguientes:

\section{Recursos públicos o concertados:}

- Unidad convalecencia sociosanitaria: Son unidades específicas para que una persona en situación de dependencia con un proceso crónico de enfermedad o patología asociada al envejecimiento reciba, una vez que está estable y a su salida del hospital, cuidados sociales y sanitarios de baja intensidad de forma simultánea mientras logra su recuperación o su rehabilitación. Para pacientes pertenecientes al HCUV, las plazas de unidad convalecencia socio sanitaria están en Valladolid. El concierto ofrece la estancia (residencial) y solo la rehabilitación motora, conllevan un copago económico y el tiempo máximo de permanencia es de 4 meses.

- Convalecencia sanitaria: Se presta asistencia sanitaria a pacientes afectos de procesos clínicos y/o patologías ya diagnosticadas con deterioro funcional previsiblemente recuperable, que precisan cuidados continuos y de rehabilitación durante la convalecencia debido a incapacidad funcional que presentan una vez superada la fase aguda de enfermedad. El concierto para acceso a estas plazas está en Valladolid, el Sistema de Salud cubre la estancia (residencial) la rehabilitación motora y la logopedia. La duración máxima es de 6 meses. El objetivo de estas plazas es la recuperación funcional, que con cuidados sanitarios subagudos les posibilite regresar a su domicilio.

- Convalecencia larga estancia: Unidad de convalecencia de larga estancia, se ofrece asistencia sanitaria, que incluye vigilancia del enfermo, control de sus procesos, diagnóstico y tratamiento de posibles complicaciones y rehabilitación. La rehabilitación que cubre el contrato es rehabilitación motora y terapia ocupacional. Es un recurso con carácter residencial, ubicado en la provincia de Burgos.

- Rehabilitación domiciliaria: Es la rehabilitación que se trata en el domicilio del 
paciente con accidente cerebrovascular, enfermedades del sistema nervioso central, lesiones de médula espinal, entre otros. Este tipo de tratamiento en el HCUV solo cubre el área motora, la rehabilitación domiciliaria pública de logopedia, neuropsicología o terapia ocupacional no existe públicamente. Además, solo llega a la capital de Valladolid y no a los pueblos. Los pacientes que viven en los pueblos que les pertenece el Hospital Clínico Universitario de Valladolid no tienen acceso a esta rehabilitación motora domiciliaria.

- Rehabilitación ambulatoria: Es la rehabilitación que se presta en el Hospital o en centros concertados de manera ambulatoria, cubre área motora y logopedia. En centros concertados está sujeta al período de financiación y el acceso a lista de espera. La rehabilitación ambulatoria en el Hospital o en un centro concertado, conlleva para pacientes dificultad de horario de ambulancias o medios de transporte (no hay línea regular para traslado a la ciudad de Valladolid desde ese pueblo y no dispone familiares que puedan proporcionar el traslado).

- Centro Estatal de Daño Cerebral (CEADAC): Es el centro de referencia nacional de Daño Cerebral, pertenece a la red de centros del IMSERSO. Ofrece una atención funcional integral y orientación a la inserción/ reinserción social. Está ubicado en la ciudad de Madrid y su acceso no le garantiza únicamente el carácter recuperable del paciente, sino la edad es un criterio de exclusión ${ }^{93}$, hasta 54 años de edad inclusive. Por ser una de sus finalidades, la integración social, el tratamiento exige que los fines de semana el paciente permanezca en su domicilio, el viernes sale del centro hasta el lunes. Lo que dificulta cumplir este requisito en pacientes que viven en viviendas con barreras o no cuentan con soporte familiar los fines de semana.

- Guttman: Es un Hospital de neurorrehabilitación. El Sistema Nacional de Salud ofrece públicamente 3 meses de tratamiento en este centro, que atiende entre las diversas patologías el ictus. $\mathrm{Su}$ tratamiento consiste en un proceso de neurorrehabilitación altamente especializado dirigido a restituir, minimizar y/o compensar las alteraciones funcionales a consecuencia del ictus. Se proporciona una atención integral, continuada, personalizada y basada en la intervención de un equipo multidisciplinar experto. El ingreso a través del Sistema Nacional de Salud, totalmente financiado, está limitado a la edad, un criterio de exclusión es la edad ${ }^{93}$, hasta los 60 años $^{168}$. Entre las normas de este centro, está la de permanecer los familiares durante los 3 meses de plaza pública cerca del paciente, mostrando 
accesibilidad y colaboración con el equipo rehabilitador. Esto supone organizar el traslado de la familia a Badalona, búsqueda de vivienda acorde con su situación económica, organización de sus responsabilidades familiares y laborales. La estancia de los familiares en Badalona les supone un gasto.

Los criterios para acceso a recursos públicos transcienden al diagnóstico clínico y pronóstico, tienen en cuenta la edad, la situación socio familiar que limita el acceso al tratamiento rehabilitador.

\section{Recursos privados:}

La TSN maneja los recursos privados a nivel local, autonómico y nacional en su gran mayoría. En su intervención social de concienciación e implicación al paciente, si éste o su familia pueden afrontar el gasto y está de acuerdo en destinarle a su máxima recuperación, se inicia el contacto con centros que cubren parte de ese tratamiento rehabilitador. Si el paciente y/o familia no pueden afrontar económicamente los tratamientos rehabilitadores que no cubre el Sistema Público de Salud, se garantiza únicamente el tratamiento rehabilitador público que si se cubre.

Por todo ello, inevitablemente, existen serias dificultades para el acceso al tratamiento rehabilitador integral propuesto por el equipo multidisciplinar:

- El tratamiento rehabilitador no está subvencionado por el Sistema Público de Salud. El paciente y la familia no puedan pagar los tratamientos económicos.

- Dificultad de acceso del paciente al tratamiento rehabilitador que se ofrece desde el hospital (no conduce, ausencia transporte público)

- No hay apoyos familiares ni formales para atender sus ABVD.

- La vivienda no cumple los criterios de habitabilidad o de deambulación

- Recurso fuera de su Comunidad Autónoma, como el Centro de Neurorrehabilitación Guttman:

-Cumple criterios, pero este recurso exige presencia familiar que no existen o no pueden desplazarse

-No puede acceder por la edad, es criterio de exclusión.

- La rehabilitación visual no está contemplada en cartera de servicios del Sistema Nacional de Salud.

- El deseo de la persona de permanecer en su entorno y en éste no se garantiza el tratamiento que precisa.

El plan rehabilitador propuesto inicialmente por el equipo multidisciplinar se ve 
condicionado por estas situaciones, fundamentalmente la limitación de acceso a recursos públicos que garanticen el tratamiento de todos los dominios afectados y en su defecto, la imposibilidad económica de afrontar los tratamientos rehabilitadores no cubiertos por el Sistema de Salud. Este plan propuesto va transformándose y culmina en el plan rehabilitador integral al que si accede el paciente.

\section{D/. Adaptación del tratamiento propuesto al tratamiento viable}

La trabajadora social comunica al resto del equipo las acciones realizadas y el resultado de su intervención:

- Explica el tratamiento rehabilitador que finalmente recibirá el paciente,

- La duración de esos tratamientos,

- Los compromisos adquiridos del paciente y la familia

- Los tratamientos prescritos que no va a recibir y los motivos (ejemplo: implican un coste que no pueden asumir o una distancia geográfica que dificulta su traslado, entre otros)

Se procede a plasmar en un documento el tratamiento rehabilitador al que, si accede el paciente, recoge: los dominios afectados que reciben tratamiento, duración del mismo, los tratamientos cubiertos por el Sistema de Salud y los privados, lugar donde recibirá el tratamiento y el tipo de tratamiento (ambulatorio o residencial), las acciones específicas en las que colaborará la familia.

Este tratamiento rehabilitador que, si recibirá el paciente, es la finalización de todo un proceso largo y complejo que parte del plan inicial rehabilitador integral propuesto por el equipo y sufre de transformación/modificaciones por los factores anteriormente descritos que condicionan la accesibilidad, queda registrado en la Historia Clínica del paciente.

\section{E/. Plan rehabilitador definitivo}

Una vez clarificado el plan rehabilitador postalta que va a recibir el paciente, la trabajadora social neurológica tramita los recursos públicos a los que tiene acceso y contacta con los recursos privados que cubren dominios afectados y que ha elegido el paciente y familia para su el tratamiento al alta. La TSN no solo gestiona el recurso que precisa al alta, sino que tiene que manejar los tiempos con precisión, pues es fundamental la disponibilidad del recurso lo antes posible para potenciar su mayor recuperación. Uno de los mayores retos es también poder incluir a los pacientes en los programas de rehabilitación en el momento adecuado ${ }^{169}$. 


\subsubsection{Alta hospitalaria}

El plan de rehabilitación integral funcional que va a recibir ya está identificado y los tratamientos gestionados. El especialista comunica a la trabajadora social neurológica que están controlados todos los aspectos médicos que impliquen un riesgo vital y es el momento de iniciar el tratamiento rehabilitador post alta. La actuación de la trabajadora social neurológica debe ser rápida y precisa, movilizando y preparando el recuso al alta, para lograr el inicio precoz de la rehabilitación que contribuya a un mayor éxito de recuperación. Sus funciones definidas al alta hospitalaria son dos:

\section{A/. Enlace y coordinación con el recurso}

La trabajadora social neurológica es el enlace y se coordina con los tratamientos rehabilitadores que va a recibir el paciente desde su destino al alta. Informa nuevamente a la familia y al paciente que retorna a su domicilio del centro (público y/o privado) dónde va a recibir la rehabilitación ambulatoria. Se insiste con carácter preferente si estos recursos tardan en empezar lo comuniquen, se debe garantizar que el inicio del tratamiento rehabilitador no se dilate en el tiempo.

\section{B/. Apoyos al alta}

Al alta hospitalaria, el paciente que retorna a su domicilio y por sus áreas afectadas, tiene pérdida de funcionalidad, en función del daño funcional ocasionado se le posibilita las ayudas técnicas que le garanticen un mínimo de funcionalidad, independencia y posibilidad desplazamiento al tratamiento rehabilitador al alta hospitalaria. Entre ellas: andador o silla de ruedas, pero también cama adaptada, silla de confort y reposapiernas.

\subsubsection{Seguimiento postalta hospitalaria}

La trabajadora social neurológica del HCUV realiza un seguimiento periódico con el paciente y familia cada 3-6 meses desde el alta hospitalaria, el tiempo de seguimiento depende de varios factores: El tiempo de permanencia en el recurso que acude al alta hospitalaria y las necesidades sobrevenidas que pueden acontecer al paciente y familia. El seguimiento tiene como objetivos:

- Conocer el nivel de funcionalidad del paciente a los meses del alta hospitalaria,

- Saber su grado de adaptabilidad a la nueva situación,

- Detectar las necesidades sociosanitarias que tiene y su nivel de cobertura,

- Explorar su estado emocional,

- Saber la organización y funcionamiento de la familia, entre otros. 
Según estas valoraciones, se reevalúa y re-enfoca el plan rehabilitador multidisciplinar establecido al alta hospitalaria. La finalidad es garantizar la continuidad asistencial del tratamiento rehabilitador ${ }^{170}$.

Este seguimiento, con la participación del paciente, la información de terceros y tener lugar con el tiempo permite identificar significativamente los impactos de una lesión, generando conocimiento sobre las brechas entre lo que se dice y lo que se hace ${ }^{148}$. Este seguimiento se realiza en una triple vertiente: con el paciente, con la familia y con otros profesionales.

\section{A/. Con el paciente}

Se mantiene entrevistas con el paciente y gestiones telefónicas para verificar la evolución funcional del mismo, su adherencia al tratamiento rehabilitador y detección de otras necesidades que puedan estar surgiendo.

Son muchos los motivos por los que el seguimiento del TSN es fundamental en pacientes que han sufrido ictus, entre ellos:

-Detección riesgos sociales que perjudican en su tratamiento rehabilitador.

- Las cargas familiares en el domicilio que hacen necesarios servicios que las cubran,

- El gasto económico del tratamiento,

- Dificultad de acudir al lugar de rehabilitación,

- El deseo/ intención de abandonar el centro,

Desmotivación por seguir con el tratamiento rehabilitador. No siempre asumen sus secuelas o las secuelas les generan en muchas ocasiones estados de frustración o derrota que pueden condicionar el abandono de dicho tratamiento

- Ausencia de apoyos formales o familiares que cubran las necesidades que la persona tiene, continuidad y evitar suspensión en el tratamiento rehabilitador.

- Necesidad de traslados continuos por rehabilitación ambulatoria, no pueden por diferentes factores mantenerse a lo largo del tiempo.

En definitiva, durante todo el proceso de seguimiento, pueden suceder acontecimientos /circunstancias inevitables que conlleven a la pérdida de la autonomía personal de la persona, lo que supone un mal funcionamiento psicosocial y se asocia a una peor calidad de vida ${ }^{171}$.

\section{B/. Con la familia}

Se mantienen entrevistas con la familia y gestiones telefónicas para verificar su estado de 
salud, los apoyos y dedicación que destina al cuidado, detección de sus necesidades personales y en el cuidado. Son muchos los motivos por los que el seguimiento del trabajo social neurológico con los familiares de pacientes que han sufrido ictus es necesario. Se muestran algunos de los motivos en la Tabla 6.

Tabla 6. Indicadores a valorar en seguimiento con familia

\begin{tabular}{|c|c|}
\hline $\begin{array}{c}\text { Indicadores de } \\
\text { riesgo }\end{array}$ & Valoración trabajo social neurológico \\
\hline $\begin{array}{l}\text { 1-Largo proceso de } \\
\text { recuperación. } \\
\text { Identificar si puede } \\
\text { provocar que la } \\
\text { convivencia sea muy } \\
\text { traumatizante para la } \\
\text { familia que soporta } \\
\text { una tensión a la que } \\
\text { no estaba preparada }\end{array}$ & $\begin{array}{l}\text { Detección y valoración: } \\
\text {-Muestra sobrecarga y el proceso rehabilitador no ha } \\
\text { finalizado (Escala Zarit) } 156 \\
\text {-Problemas familiares, sentimientos de frustración, } \\
\text { ansiedad, inseguridad, que generan situaciones de mucho } \\
\text { estrés y origina disfunciones que perjudican proceso } \\
\text { rehabilitador. } \\
\text {-Posible claudicación familiar. }\end{array}$ \\
\hline $\begin{array}{l}\text { 2-Deterioro } \\
\text { funcional y } \\
\text { emocional en el } \\
\text { cuidador familiar }\end{array}$ & $\begin{array}{l}\text { Detección y valoración: } \\
\text {-Afectación de su estado de salud, su bienestar y } \\
\text { sentimiento de incapacidad de afrontar esta } \\
\text { responsabilidad, pasando a constituir para el área sanitaria } \\
\text { un paciente "oculto" o "desconocido", que requiere de un } \\
\text { diagnóstico precoz e intervención inmediata, antes de } \\
\text { presentar el Síndrome del Cuidador }{ }^{172} \text {. }\end{array}$ \\
\hline $\begin{array}{l}\text { 3-Su funcionamiento } \\
\text { social presenta } \\
\text { signos de deterioro. }\end{array}$ & $\begin{array}{l}\text { Detección y valoración: } \\
\text {-Los roles se invierten con frecuencia } \\
\text {-Se evaden responsabilidades } \\
\text {-Los compromisos en el cuidado no se respetan }\end{array}$ \\
\hline $\begin{array}{l}\text { 4-La familia se } \\
\text { siente sola y } \\
\text { desamparada, sus } \\
\text { fuerzas van } \\
\text { flaqueando. }\end{array}$ & $\begin{array}{l}\text {-Reducción de salidas fuera del domicilio } \\
\text {-Reducción de contactos con amigos y vecinos } \\
\text {-No acuden a ninguna actividad } \\
\text {-Sus salidas se limitan a consultas médicas del familiar } \\
\text { (pasando a un plano secundario las suyas), a compras de } \\
\text { alimentación. }\end{array}$ \\
\hline
\end{tabular}

Fuente: Elaboración propia

Dependiendo cual sea la situación clínica del paciente (recuperación temporal, cronificación), la posición de los diferentes miembros ira volviendo a los parámetros previos a la enfermedad, o requerirá modificaciones permanentes (particularmente, si el resultado es una situación de incapacidad que conlleve ciertos niveles de dependencia) ${ }^{173}$. En definitiva, mantener controlado el conocimiento de las capacidades y limitaciones que durante el proceso rehabilitador presenta la familia, para darle el soporte preciso que van 
necesitando y contribuir a su calidad de vida y la del paciente.

\section{C/. Con los profesionales}

La coordinación con diferentes profesionales y los recursos de la comunidad se hace imprescindible para garantizar la continuidad en la atención socio sanitaria. La continuidad asistencial es considerada uno de los componentes fundamentales para el logro de la "calidad en la atención sanitaria"174.

Una coordinación con los diferentes profesionales del equipo del HCUV y de los diferentes dispositivos de rehabilitación que van interviniendo con el paciente. Se muestra en la siguiente tabla.

Tabla 7. Coordinación con diferentes profesionales

\begin{tabular}{|c|c|}
\hline Coordinación & Objetivos de Trabajo Social Neurológico \\
\hline $\begin{array}{l}\text { 1-Profesional } \\
\text { centro } \\
\text { rehabilitador } \\
\text { A través de } \\
\text { gestiones } \\
\text { telefónicas o } \\
\text { mails, reuniones } \\
\text { en el centro. }\end{array}$ & $\begin{array}{l}\text { - Conocer su evolución clínico-social } \\
\text { - Identificar su nivel de adaptabilidad en el centro y } \\
\text { colaboración en su proceso de recuperación. } \\
\text { - Controlar el alta del centro para proporcionar y organizar } \\
\text { continuidad del tratamiento rehabilitador si precisa (se } \\
\text { recibe el informe al alta que determina si precisaría de más } \\
\text { tratamiento rehabilitador). } \\
\text { Recepcionar el informe al alta del centro de rehabilitación por } \\
\text { el que pasa y puesta en conocimiento del equipo neurológico } \\
\text { del HCUV }\end{array}$ \\
\hline \multicolumn{2}{|c|}{$\begin{array}{l}\text { - Se mantiene una comunicación fluida con ellos para el buen desarrollo del caso, } \\
\text { sin interferirnos unos con otros en el trabajo }{ }^{175} \text {. En definitiva, una intervención } \\
\text { en red, interacción de profesionales, pacientes y organizaciones del ámbito } \\
\text { sanitario y socio sanitario, con otros sistemas de protección. }\end{array}$} \\
\hline $\begin{array}{l}\text { 2-Equipo } \\
\text { neurológico }\end{array}$ & $\begin{array}{l}\text { - Comunicación continúa con el equipo neurológico sobre la } \\
\text { evolución clínico-social del paciente } \\
\text { - Informa y facilita el informe de alta del centro de } \\
\text { rehabilitación al equipo neurológico. } \\
\text { - Organizar el seguimiento con el equipo neurológico al alta } \\
\text { del centro recuperación y conoce por el mismo su situación } \\
\text { clínica. } \\
\text { - Rediseñar conjuntamente el tratamiento rehabilitador que va } \\
\text { necesitando. } \\
\text { A lo largo del seguimiento puede verificarse / evidenciarse que } \\
\text { la persona no ha recuperado la totalidad de las funciones } \\
\text { afectadas y precise de continuidad del tratamiento } \\
\text { rehabilitador, analizando de nuevo áreas afectadas, tratamiento } \\
\text { que precisa, posibilidades públicas actuales según su situación } \\
\text { sanitaria, socioeconómica y personal. }\end{array}$ \\
\hline
\end{tabular}

Fuente: Elaboración propia 


\subsubsection{Reinserción socio profesional del paciente}

Durante el seguimiento que se realiza con el paciente y familia al alta hospitalaria y durante el tratamiento rehabilitador integral, puede comprobarse que el paciente ha quedado con secuelas pero que no va a recuperar más, lo que influye en el funcionamiento social, el mantenimiento de la salud y, por tanto, en su reinserción social La trabajadora social neurológica, en base a la situación personal y los déficits persistentes tras la finalización de su proceso de rehabilitación funcional, realiza las siguientes funciones:

- Establece e identifica prioridades de acción y se definen metas a alcanzar con el fin de evitar el aislamiento social de la persona y el deterioro de su salud.

- Interviene con la finalidad de contribuir a rescatar sus capacidades y no acentuar la discapacidad: que comprendan, manejen y hagan frente con éxito a los impedimentos duraderos de su enfermedad.

- Proporciona rehabilitación psicosocial, destinada a capacitar a la persona mediante entrenamientos específicos, instauración de hábitos, o bien ofertando soporte social necesario para lograr un funcionamiento mínimamente aceptable.

- Se orienta al paciente y familiar, en el reconocimiento de su incapacidad laboral

Se asesora en su camino hacia la inserción profesional:

* Orientar hacia servicios especializados para personas con discapacidad.

* Encaminarle a la realización de formación profesional para que la persona con discapacidad se capacite

El objetivo de todas estas intervenciones es contribuir a la integración de la persona en su medio y para ello es fundamental contar con el soporte formal, familiar, relacional, residencial que lo permita.

Por tanto, el tratamiento del trabajo social es una parte significativa del programa de rehabilitación de accidentes cerebrovasculares. Se favorece al paciente, a su familia y al equipo de rehabilitación a trabajar juntos para alcanzar metas realistas, determinadas individualmente. Los conocimientos y habilidades de la trabajadora social neurológica están dirigidos a asistir al paciente y a su familia en el proceso de ajuste y a facilitar el regreso del paciente que ha sufrido accidente cerebrovascular a la comunidad al más alto nivel posible de funcionamiento emocional, social y económico ${ }^{176}$. 


\section{JUSTIFICACIÓN DE LA INVESTIGACIÓN}

La investigación, realizada durante 5 años, se origina principalmente por las siguientes causas/preocupaciones:

1. El problema que se plantea, el tratamiento rehabilitador integral post-ictus para la persona que lo ha sufrido, es un problema prioritario para la sociedad, que no está resuelto y es necesario investigar. El ictus afecta a una cuarta parte de la población, siendo la principal causa en España de discapacidad en adultos ${ }^{10}$.

2. Los factores sociales han sido poco estudiados en el pronóstico del ictus en nuestro medio y por tanto se desconoce en gran medida su importancia para determinar el acceso a la RHB integral. Asimismo, se ignora hasta qué punto la labor del trabajo social neurológico es importante a la hora de facilitar el acceso a la RHB integral teniendo en cuenta dichos factores sociales. En el año $2025^{26}$, de no evitarse, más de 500.000 personas tendrán discapacidad, una cifra considerable para plantearse evaluar esto.

3. La RHB integral, aceptada de forma teórica, es un modelo que no ha conseguido implantarse en la práctica clínica real de los pacientes con ictus. Para lograrlo, en primer lugar, es necesario evaluar su impacto sobre el pronóstico funcional y la vida de las personas a largo plazo. Y, posteriormente, intentar averiguar cuáles son las barreras que están dificultando más su implantación en el proceso de cuidados postictus. Los actuales Sistemas de Salud adolecen de un seguimiento continuo. El apoyo y seguimiento a largo plazo continuos son insuficientes en muchas partes de Europa, pero resultan imprescindibles en la RHB integral del paciente con ictus. Por ejemplo, el tratamiento rehabilitador domiciliario de los pacientes leves o moderadamente afectados ha sido demostrado por diversos estudios que durante los tres primeros meses del ictus iguala en resultados a los tratados en un Hospital o unidad especializada, produce una reducción del $52 \%$ de las hospitalizaciones requeridas por los pacientes, una vez superada la fase aguda y una considerable reducción en el uso de cuidados sanitarios ${ }^{177}$. Sin embargo, no se recibe en todas las localidades donde residen los pacientes que la precisan.

La investigación aporta novedades en este campo, el análisis pormenorizado de los diferentes factores socioambientales que influyen en el acceso al tratamiento RHB integral post-ictus de pacientes que la precisan, así como la evaluación a largo plazo del impacto de la RHB integral post-ictus en la autonomía funcional del paciente. 


\section{Capítulo 2. HIPÓTESIS Y OBJETIVOS}




\section{HIPÓTESIS}

La supervivencia por ictus ha mejorado considerablemente, pero las secuelas por la discapacidad persisten ${ }^{9}$. La evidencia científica, cuya revisión se ha realizado a lo largo de la tesis, señala el beneficio que ocasionaría el tratamiento de las secuelas y déficits provocadas por el ictus a través de una rehabilitación multidisciplinar que incluya todas las áreas afectadas.

Las hipótesis de las investigaciones realizadas en el marco de la presente tesis son tres:

- H.1. Existe una proporción importante de los pacientes con ictus que, pese haber sido considerados candidatos a RHB integral post-ictus, no la reciben

- H.2. Entre las barreras que impiden el acceso a la RHB integral a los pacientes con ictus candidatos a la misma, las de índole social y económica tienen gran importancia, por lo que se asociarán a un peor acceso a la RHB integral.

- H.3. El acceso a la RHB integral se asociará a un mejor pronóstico funcional a largo plazo de los pacientes con ictus candidatos a recibirla.

Para comprobar la verosimilitud de estas hipótesis, se plantean 2 grandes preguntas de investigación, a las que la tesis pretende dar respuesta:

1. ¿Que ha implicado el acceso al tratamiento rehabilitador para la recuperación funcional del paciente?

2. ¿Qué factores personales, familiares y sociales pueden determinar y/o condicionar el acceso a la rehabilitación integral post-ictus, especialmente en contextos donde no hay centros especializados en dicha rehabilitación?

\section{OBJETIVOS}

Objetivo general 1: Conocer la probabilidad que tienen los pacientes con ictus de acceder a la RHB integral tras el alta hospitalaria, habiendo sido considerados candidatos a recibirla por un equipo multidisciplinar durante el ingreso

Objetivo general 2: Analizar los factores personales, familiares y sociales que influyen en el acceso a la rehabilitación integral de pacientes con ictus.

Objetivo general 3: Evaluar los efectos a largo plazo de la rehabilitación integral postictus en la autonomía funcional de los pacientes que la han recibido. 


\section{Capítulo 3. MATERIAL Y MÉTODOS}


Este apartado incluye las reproducciones de los contenidos de los artículos originales de investigación que integran la Tesis Doctoral.

El proceso de investigación se basa en dos estudios relacionados y sucesivos: cada uno de ellos ha consistido en un estudio específico, con un diseño metodológico propio. El segundo estudio se planteó y diseñó teniendo en cuenta los resultados obtenidos en el primero.

\section{1. ÁMBITO DE ESTUDIO Y DISEÑO METODOLÓGICO}

Primer estudio: Análisis de los factores asociados a la recuperación integral de pacientes de ictus al alta de Hospital de Agudos. El ámbito de estudio corresponde a los pacientes ingresados en el año 2016 en el Servicio de Neurología del Hospital Clínico Universitario de Valladolid por ictus isquémico y hemorrágico mayor de 18 años que precisaban de rehabilitación integral.

Segundo estudio: Estudio del impacto de la RHB integral sobre la recuperación funcional a largo plazo de los pacientes con ictus. Analizados los resultados obtenidos en el primer estudio, se abordó un segundo estudio con pacientes que ingresan en el HCUV en el año 2015 por ictus isquémicos mayores de 18 años. Se amplía con respecto al estudio anterior, las variables de influencia al acceso del tratamiento rehabilitador integral y se analizan los efectos del tratamiento rehabilitador a largo plazo ( 5 años desde el ictus) en la recuperación funcional de los pacientes que han sufrido ictus.

Ambos estudios son retrospectivos, longitudinales en el tiempo (se analiza en el presente, con datos del pasado), siendo su inicio posterior a los hechos estudiados. Son estudios observacionales unicéntricos, retrospectivos, longitudinales de seguimiento a largo plazo.

\section{SELECCIÓN DE PACIENTES/POBLACIÓN DE ESTUDIO}

Los pacientes de las respectivas muestras fueron identificados por el Especialista de Neurología, que al ingreso hospitalario solicito valoración de la trabajadora social del Servicio Médico.

La base de datos esta anonimizada y no existen en ella elementos que permitan la identificación del paciente, en cumplimiento de la Ley Orgánica de Protección de $\operatorname{Datos}^{178}$. 
Los estudios de esta investigación fueron aprobados por el Comité de Ética de Investigación Clínica del Hospital de Referencia. El código de aprobación del primer estudio: PI 17-776 y el código de aprobación del segundo estudio: PI 20-1671.

\subsection{Criterios de inclusión y exclusión}

Los criterios de inclusión y exclusión de ambos estudios están recogidos en la siguiente Tabla 8.

Tabla 8. Criterios de inclusión y exclusión ambos estudios.

\begin{tabular}{|l|l|l|}
\hline \multicolumn{2}{|c|}{ Criterios de inclusión } & \multicolumn{1}{c|}{ Criterios de exclusión } \\
\hline Primer Estudio & Segundo Estudio & Primer y segundo Estudio \\
\hline $\begin{array}{l}\text { Mayores de } 18 \\
\text { años con ictus } \\
\text { isquémico y/o } \\
\text { hemorrágico }\end{array}$ & $\begin{array}{l}\text { Mayores de } 18 \\
\text { años con ictus } \\
\text { isquémico }\end{array}$ & Se encuentra en fase terminal \\
\hline $\begin{array}{l}\text { Capacidad cognitiva preservada para } \\
\text { ejecutar órdenes tratamiento } \\
\text { rehabilitador }\end{array}$ & $\begin{array}{l}\text { Deterioro cognitivo que impide abordaje } \\
\text { tratamiento rehabilitador. }\end{array}$ \\
\hline $\begin{array}{l}\text { Tras valoración por un equipo } \\
\text { interdisciplinar: Neurólogo, } \\
\text { Rehabilitador, Fisioterapeuta, } \\
\text { Logopeda, Enfermería y Trabajo } \\
\begin{array}{l}\text { Social, perspectiva de recuperación } \\
\text { funcional total o parcial }\end{array}\end{array}$ & $\begin{array}{l}\text { Presencia de otra patología que prevalece y } \\
\text { ocasiona otro tipo de necesidades socio } \\
\text { sanitarias }\end{array}$ \\
\hline $\begin{array}{l}\text { Más de 2 áreas/dominios afectados: } \\
\text { motor, lenguaje/deglución, visual, } \\
\text { emocional, etc. }\end{array}$ & Fallecimiento en el ingreso hospitalario \\
\hline $\begin{array}{l}\text { Residentes en Área de Salud } \\
\text { Valladolid Este }\end{array}$ & Residentes en otra Área de Salud. \\
\hline $\begin{array}{l}\text { Rankin }{ }^{160} \geq 1 \text { al alta hospitalaria } \\
\text { Rankin 0 }\end{array}$ \\
\hline
\end{tabular}

Fuente: Elaboración propia 2021

\subsection{Tamaño muestral}

El tamaño muestral del primer estudio comprende los pacientes ingresados desde enero 2016 hasta 31 diciembre del 2016 que cumplían los criterios anteriores. Se han incluido un total de 102 pacientes.

En el segundo estudio el tamaño muestral corresponde a los ingresados durante el año 2015, que cumplen los criterios anteriormente descritos. Se han incluido un total de 171 
pacientes. Este segundo estudio finalizó en marzo del 2020, confirmándose que 137 pacientes habían sobrevivido sobre los que se analizó los efectos a largo plazo del tratamiento rehabilitador integral

\section{PROTOCOLO DE RECOGIDA DE DATOS Y SEGUIMIENTO}

A la autorización de la investigación, se comenzó con el diseño de la herramienta de recogida de datos y el registro de información que consta de 4 fases (ingreso, alta hospitalaria, seguimiento a los 3 meses y a los 5 años).

\subsubsection{Base de datos}

Se diseñó una base de datos diferente para cada estudio de investigación, ambas recogen variables de datos clínicos, epidemiológicos y sociales, se verán en el apartado de variables de estudio.

En el primer estudio: Análisis de los factores asociados a la recuperación integral de pacientes de ictus al alta de Hospital de Agudos. Se estudian edad, sexo, áreas afectadas, necesidad apoyos al alta, familia, localidad (zona rural y urbana), situación económica, Rankin, NIHSS, entre otros.

En el segundo estudio: Estudio del impacto de la RHB integral sobre la recuperación funcional a largo plazo de los pacientes con ictus. Se amplían las variables de acceso al tratamiento rehabilitador y se añaden variables que miden los efectos a largo plazo. Se ha realizado un análisis exhaustivo a través de un registro sistemático de pacientes que precisan de rehabilitación integral atendidos por trabajo social neurológico del HCUV y seguimiento a largo plazo para conocer efectos en sus vidas.

\subsection{Recogida de los datos}

Los datos obtenidos y registrados en la base de datos son los siguientes:

Situación clínica del paciente. Se comprueban las exploraciones neurológicas realizadas, tratamiento durante el ingreso hospitalario, el que precisa al alta, juicio clínico, evolución y pronóstico al alta hospitalaria.

Estos datos se han obtenido a través de las diferentes herramientas informáticas que se manejan en el HCUV (Gacela, Informes Clínicos, Jimena), Medora (Atención Primaria de Salud) y, además se ha aplicado el Test Apgar Familiar ${ }^{132}$, Escala Gijón ${ }^{155}$, Índice Barthel $^{152}$, NIHSS ${ }^{159}$ y Rankin ${ }^{160}$ para analizar con precisión las diferentes variables objeto de estudio. Se ha consultado la historia clínica en papel del paciente, mantenido 
entrevistas estructuradas con el paciente, aplicación NIHSS y Rankin, reuniones semanales con equipo interdisciplinar, informes de alta de los recursos de rehabilitación. Situación sociofamiliar se ha determinado mediante la aplicación de la escala Apgar familiar, entrevistas estructuradas presenciales y telefónicas con paciente y familia, aplicación Escala Gijón, comprobación volante de empadronamiento y certificado de convivencia.

En relación con el tratamiento rehabilitador que está recibiendo, se ha consultado el informe del especialista y trabajador social del centro donde le recibe, gestiones telefónicas con los mismos, se ha comprobado la distancia en kilómetros del domicilio al centro, las terapias que recibe, tipología del recurso y su modalidad de pago (privada/publica/copago)

La metodología utilizada en esta investigación es de carácter cuantitativo, se han analizado los datos seleccionados en la base de datos y se utilizado las herramientas informáticas para la sistematización de los datos.

\subsection{Variables de estudio}

Primer estudio: Análisis de los factores asociados a la recuperación integral de pacientes de ictus al alta de Hospital de Agudos

Se recogieron variables personales (edad, sexo, estado civil, ingresos económicos, lugar de residencia).

-La edad. Se comprobó a través del carné de identidad. Se codificó en tres intervalos.

-El sexo. Las dos categorías establecidas son hombre y mujer

-EI estado civil, se ha categorizado en casado, soltero, divorciado/separado y viudo. Se comprobó el libro de familia del paciente y /o sentencia de separación/divorcio.

-Los ingresos económicos unidad familiar de convivencia se han comprobado a través: nómina, declaración jurada o extracto bancario. Se ha calculado la media de ingresos económicos de la unidad familiar de convivencia.

-El lugar de residencia, se ha codificado por zona urbana o rural (municipios de la provincia de Valladolid menores de 20000 habitantes). Se comprobó el volante de empadronamiento.

Se recogieron variables familiares:

-Apoyo Familiar. La identificación de si existe o no apoyo familiar, hace necesario definir qué se entiende por apoyo familiar, que el marco de esta investigación será la 
"provisión de recursos de tipo instrumental, emocional, afectivo o económico que recibe una persona de su núcleo familiar" ${ }^{179}$.

Se aplicó la Escala Apgar Familiar ${ }^{132}$, se codifico: SI/NO apoyo Familiar.

1. SI Apoyo Familiar. Función Familiar Normal: 17-20 puntos.

2. NO Apoyo Familiar. Disfunciones Familiares: 9-16 puntos.

Se analizó la influencia de estas variables en el acceso a la rehabilitación integral.

Segundo estudio: Estudio del impacto de la RHB integral sobre la recuperación funcional a largo plazo de los pacientes con ictus.

Se estudiaron las del primer estudio y se ampliaron variables de acceso a la rehabilitación integral, en las siguientes:

\subsubsection{Variables personales}

- La actividad laboral, se categorizo en:

1. SI/NO Jubilado. Se ha comprobado con certificado de vida laboral

- La accesibilidad de la vivienda se ha codificado en base a: Adecuada (el paciente puede permanecer en la misma y deambular sin dificultad), barreras (el paciente $\mathrm{n}$ puede deambular con facilidad en el misma), escaleras (la vivienda tiene escaleras de acceso a la misma y/o a otras plantas de la vivienda).

Esta información se ha sido verificada a través de entrevista paciente y familia, contacto con profesionales de su centro de salud y acción social, y en algunos casos de los vecinos que conocían el inmueble.

- La propiedad de la vivienda se ha codificado en $\mathrm{Si} / \mathrm{NO}$ en su propiedad. Se ha comprobado por el certificado catastral, en los casos que la propiedad no pertenecía al paciente se ha verificado con el pertinente contrato de alquiler o una declaración jurada familiares de estar cedida al uso.

- El régimen de la vivienda se ha codificado en 3 categorías: Propiedad (la vivienda es propiedad del paciente), alquiler y cedida al uso (dispone del uso de la vivienda)

Se ha comprobado a través certificado catastral, contrato alquiler y declaración jurada.

- La unidad de convivencia se refiere a las personas que viven en el mismo domicilio, se ha comprobado en la entrevista mantenida con paciente y a través del certificado de convivencia.

- La variable Rankin, Valor de la escala modificada de Rankin previa al ictus y en el momento del alta de hospitalización. 
- La variable NIHSS al alta hospitalaria, se ha codificado en: Leve (1), moderado (2), grave $(3)^{159}$. Se ha determinado a través de la escala NIHSS ${ }^{159}$.

- El tratamiento rehabilitador que precisa se ha codificado en base al tratamiento que precisaba: Fisioterapia (rehabilitación motora), logopedia (rehabilitación lenguaje y/o deglución), terapia ocupacional, rehabilitación visual, neuropsicología, auxiliar para atención ABVD, Salud Mental.

Se ha establecido y determinado según el equipo multidisciplinar que valora el tratamiento precisa el paciente.

- La variable modalidad de tratamiento, se ha codificado en: Tratamiento público (abonado en su totalidad por la Administración), concertado (el paciente realiza un copago/aportación económica) y privado (aportación privada para pago tratamiento)

- La variable, factores de riesgo, se ha codificado en: Hipertensión Arterial, Diabetes Mellitus, Ansiedad, Depresión, Bebedor de Riesgo, Insomnio, Tabaquismo Activo.

Se han comprobado los factores de riesgo a través de las reuniones equipo interdisciplinar, informe e historia clínicos de Atención Primaria.

\subsubsection{Variables familiares}

Los factores familiares que se van a investigar para conocer e identificar su influencia en el acceso a la rehabilitación integral son: la existencia de familia, si el paciente cuenta con apoyo familiar y si existía presencia de cuidador familiar antes del paciente sufrir ictus.

- La existencia de familiares se ha comprobado a través entrevista con el paciente y verificación mediante el libro de familia, en caso de cónyuge e hijos

La comprobación de los familiares que viven en el mismo domicilio se ha verificado inicialmente a través de entrevista al paciente y familiares, petición certificado empadronamiento y convivencia.

- Administración del test Apgar Familiar ${ }^{132}$ : Aplicación al paciente y al familiar que se ha identificado previamente como el más cercano y garante de apoyo familiar al paciente. Es un cuestionario de cinco preguntas que evidencia el estado funcional de la familia.

- Finalmente, se ha verificado la existencia o no de cuidador familiar que garantice la atención que precisa el paciente, teniendo en cuenta las condiciones de idoneidad de este y la asunción de la responsabilidad del cuidado de la persona dependiente. 
La presencia de cuidador familiar se codifico en SI/NO, se ha verificado la presencia de esta figura a través entrevista con paciente y contacto con vecinos.

Se comprobó si había cuidador familiar anterior al ictus, pero solo en 2 casos de los 9 pacientes que antes del ingreso hospitalario tenían limitaciones funcionales se confirmó, un dato que se desechó para analizarle en la investigación.

\subsubsection{Variables sociales}

- La variable de apoyo social se codifico inicialmente en: Amigos, amigos y familia a partir segundo grado consanguinidad o afinidad, amigos y vecinos (sale de casa), amigos y vecinos (no sale de casa), amigos y voluntariado, es voluntario, familia a partir segundo grado consanguinidad o afinidad, familia y vecinos, no tiene apoyo, voluntariado. Se comprobó a través entrevista con el paciente, familia, vecinos de apoyo, entidades de voluntariado y declaraciones juradas por estas personas.

Posteriormente a la obtención de los datos anteriores, se mantuvo otra entrevista con el paciente para analizarles y determinar si consideraba SI/NO contaba con Apoyo Social. La codificación se estableció finalmente en SI/NO Apoyo Social.

- La variable riesgo social se ha codificado, SI/NO riesgo social, determinado el riesgo social a través de la aplicación de la Escala Gijón ${ }^{155}$.

- La variable intervención social anterior por trabajo social de Centro de Salud o Servicios Sociales, se ha comprobado a través de entrevista con familiares, coordinación con esos profesionales y comprobación en programa informático de Atención Primaria, Medora. Se ha codificado, SI/NO intervención social anterior al ingreso hospitalario.

- La variable reconocimiento de valoración de dependencia antes del ingreso hospitalario. Se ha comprobado a través de comunicación con Sección Dependencia, resolución de dependencia y entrevista con familia. Se codifico en: NO reconocido, Grado 0, Grado 1, Grado 2, Grado 3 y en trámite.

\subsubsection{Variable rehabilitación integral}

La variable rehabilitación integral se ha codificado en SI/NO accede a la rehabilitación integral. La afirmación de SI/NO se ha establecido en base a la rehabilitación integral que precisa el paciente, valorada por el equipo multidisciplinar:

1. Áreas afectadas: lenguaje, deglución, motora, cognitiva, movimiento, emocional.

2. Identificación del tratamiento rehabilitador integral que precisa. 
(Neuropsicología, logopedia, terapia ocupacional, etc.): Se accede al informe clínico del paciente que señala su juicio clínico, áreas afectadas, tratamiento preciso, al dictamen de valoración del equipo clínico especializad. A través del profesional que tramita el recurso, trabajo social de neurología, comprobación si va a recibir el tratamiento rehabilitador completo y coordinación paciente-familia y equipo sigue el tratamiento:

3. Tratamiento rehabilitador administrado al alta hospitalaria. A los 3 meses se comprobó que recibían tratamiento rehabilitador en todas las áreas funcionales en las que presentaban déficit.

Segunda fase: Estudio del impacto de la RHB integral sobre la recuperación funcional a largo plazo de los pacientes con ictus

Las variables son las siguientes:

La variable situación funcional a largo plazo: Se comprobó la autonomía funcional de los pacientes supervivientes a través de la aplicación del Índice Barthel. Se aplicó el Índice Barthel al alta hospitalaria y posteriormente a los 5 años, para conocer el resultado del tratamiento rehabilitador recibido en este tiempo de los pacientes que accedieron en comparación con los que no accedieron al tratamiento rehabilitador integral. El IB se evaluó en el momento del alta hospitalaria y a los 5 años. De esta manera, es posible determinar cuál es el estado funcional del paciente y cómo ha progresado hacia la independencia

Se codificó según estudio ${ }^{180}$ publicado en revista de la Sociedad Española de Neurología, agrupándose por categorías de dependencia:

1. Dependencia total (puntuación 0-20);

2. Dependencia grave $(>20-40)$;

3. Dependencia moderada $(>40-60)$;

4. Dependencia leve $(>60-90)$

5. Independencia ( $>90-100)$.

\subsection{Descripción del proceso: Temporalidad}

Tanto en la primera como en la segunda investigación se ha establecido un proceso secuencial de recogida de variables en diferentes fases. 


\subsubsection{Valoración inicial al ingreso hospitalario:}

La valoración inicial de la trabajadora social de neurología solicitada por el equipo de neurología del paciente que ingresa por ictus isquémico se realizó entre las 24-48 horas desde la solicitud, con el objetivo de conocer los daños/secuelas, factores sociofamiliares y tratamiento rehabilitador preciso.

Los datos iniciales recogidos en esta primera valoración se refieren a las variables expuestas anteriormente.

\subsubsection{Valoración al alta hospitalaria:}

Cuando el neurólogo comunica el alta hospitalaria, se realiza otra segunda valoración en la que se recogen en la base de datos las siguientes variables: Rankin y NIHSS alta, puntuación Índice Barthel, destino al alta hospitalaria, tratamiento que necesita y cuál va a recibir, modalidad de tratamiento (público, concertado o privado), distancia al centro rehabilitador desde el domicilio.

\subsubsection{Valoración a los 3 meses:}

A los 3 meses se comprueba con el paciente y con el centro de destino al alta hospitalario, si le está recibiendo y específicamente que tratamientos. En esta visita se determina SI han accedido o NO a la Rehabilitación Integral que precisaban.

\subsubsection{Valoración a los 5 años:}

En el segundo estudio (Estudio del impacto de la RHB integral sobre la recuperación funcional a largo plazo de los pacientes con ictus) se pretende analizar la repercusión a largo plazo en las actividades de la vida diaria de la población objeto de nuestro estudio de la rehabilitación integral frente a los que no la han recibido al alta hospitalaria en el año 2015 por ictus isquémico.

Para ello, se ha comprobado desde febrero hasta el 10 de marzo del 2020, los pacientes fallecidos de la muestra inicial de 171 pacientes, a través de certificado de defunción, comprobación en historia clínica y contacto telefónico con familiares.

Los pacientes que han sobrevivido a marzo de 2020 son 137, se ha analizado la siguiente variable pronostica: La recuperación funcional a largo plazo de los pacientes con ictus. 


\section{INSTRUMENTOS DE MEDIDA/ESCALAS UTILIZADAS}

\subsection{Nihss}

El estado neurológico se determina generalmente a través de la escala para el ictus del Instituto de Salud de los EU (NIHSS), debido a que ha sido el instrumento de evaluación neurológica más utilizada en ensayos clínicos aleatorizados (ECA) sobre ictus en los últimos 10 años ${ }^{181}$.

Es la escala más utilizada para valorar la gravedad del ictus. Tiene 15 ítems y aporta información en relación con el déficit neurológico: es de reconocida validez y reproductibilidad y predice el resultado a largo plazo. Se trata de la escala que define mejor la gravedad inicial del ictus y tiene mayor valor predictivo.

Está validada al español en el año $2006^{159}$ y su validez ha sido constatada mediante diversos estudios $^{182}$. La categorización de la Escala NIHSS utilizada para este estudio es la que establece los siguientes rangos ${ }^{183}$ : NIHSS $<4$ : LEVE, NIHSS $<16$ : MODERADO y NIHSS>16:GRAVE.

\subsection{Escala Modificada de Rankin (mRs)}

Existen diversos trabajos en la bibliografía que han puesto de manifiesto la validez de esta escala. Se trata de un instrumento importante para la valoración de la recuperación del ictus. Está compuesta por unos grados bien definidos y fáciles de entender que describen la discapacidad global. Mide la independencia funcional en una escala de siete grados basados en el nivel de independencia y teniendo de referencia el nivel de independencia previa al ictus. Una puntuación de 1 en la escala modificada de Rankin (mRs) original significa no tener discapacidad y 5 suponía el nivel más alto de discapacidad (requiere atención constante). Van Swieten ${ }^{184}$ incluyeron en la escala el nivel 0 (no síntomas).

La forma convencional de administración es mediante un proceso de entrevista guiada. Entre las ventajas de la escala modificada de Rankin (mRs) se encuentra que es una medida extremadamente sencilla y es una herramienta muy útil para categorizar los resultados de nivel funcional. Existe una correlación fuerte de esta escala con otros instrumentos de medida de gravedad del ictus, aunque puede verse modificada por factores socioeconómicos y por la comorbilidad del paciente ${ }^{58}$. La mRs pone especial énfasis en la capacidad del paciente para caminar. Raymond en 2008 estableció la siguiente categorización de la escala ${ }^{185}: \mathrm{mRs} \leq 2$ : no discapacidad, $\mathrm{mRs}>2$ : discapacidad. 
Se tomó como referencia en este estudio, codificando $\leq 2$ y los que si discapacidad en Rankin 3, 4 y 5.

\subsection{Escala Gijón}

Otro aspecto fundamental es trascender al enfoque de intervención sustentado principalmente en la enfermedad, para ampliar el análisis de aspectos sociales y familiares que se correlacionan de manera directa con la funcionalidad física y cognitiva de las personas mayores, donde la valoración socio familiar permite a los equipos médicos y de asistencia social, identificar la calidad de apoyo y las estrategias con el fin de establecer medidas de intervención oportunas.

La escala de valoración socio familiar de GIJÓN constituye un instrumento que ha permitido la detección de situaciones de riesgo o problemática social con un grado de fiabilidad alto y validez aceptable, con experiencias favorables en su implementación en servicios asistenciales de salud para orientar la identificación oportuna de necesidades socio familiares en el paciente mayor hospitalizado ${ }^{186}$. Además, la escala Gijón contempla en su valoración la vivienda, situación económica, relaciones sociales, apoyo red social y apoyo familiar ${ }^{155} 156$.

La escala de valoración sociofamiliar (abreviada y modificada de GIJÓN) ${ }^{155}$. Creada a final de los años 90. El cuestionario recoge aspectos sobre las condiciones del entorno. En la escala de valoración de déficit sociofamiliares los resultados son los siguientes ${ }^{\mathbf{1 5 5}}$ 156: < 10 puntos: Buena o aceptable situación social, 10-14 puntos: Riesgo social y > 15 puntos: Problema social.

La codificación en esta investigación se determinó: SI Riesgo Social (10-15 puntos) y NO Riesgo Social $(<10$ puntos).

\subsection{Apgar familiar}

Se ha seleccionado el Test Apgar Familiar ${ }^{132}$ porque identifica 5 de las funciones familiares que consideramos fundamentales valorar:

1. Adaptación: Capacidad de usar recursos intra y extra familiares a fin de resolver problemas en situación de crisis

2. Participación: Implicación de los miembros en la toma de decisiones y responsabilidades familiares

3. Gradiante de recurso personal: es el desarrollo de la maduración física, emocional y auto realización que alcanzan los componentes de una familia gracias a su apoyo 
y asesoramiento mutuo.

4. Afectividad: Relación de amor, cariño, respeto y atención existente entre los miembros familiares

5. Recursos: Es el compromiso de dedicar tiempo a atender las necesidades físicas y emocionales de otros miembros de la familia, generalmente implica compartir unos ingresos económicos, tiempo y espacios.

\section{5. Índice Barthel}

El índice de Barthel (IB) se utiliza desde 1955 en hospitales de enfermedades crónicas de Maryland $^{187}$. (Hospital del Estado de Montebello, Hospital Principal del Ciervo, y Hospital Occidental de Maryland). Mide la independencia funcional en el cuidado personal y la movilidad del paciente ${ }^{188}$, valorando la capacidad de una persona para realizar diez actividades de la vida diaria (AVD) consideradas como básicas, obteniéndose una estimación cuantitativa de su grado de independencia.

El IB, también conocido como "Índice de Discapacidad de Maryland", se define como: "Medida genérica que valora el nivel de independencia del paciente con respecto a la realización de algunas actividades de la vida diaria (AVD), mediante la cual se asignan diferentes puntuaciones y ponderaciones según la capacidad del sujeto examinado para llevar a cabo estas actividades"187.

El IB es un instrumento de gran utilidad en la rehabilitación, por su validez y fiabilidad, es fácil de aplicar y de interpretar. Su aplicación es de bajo costo y es útil para dar seguimiento a la evolución de los pacientes. Las molestias son escasas, pues requiere que el paciente realice algunas actividades básicas o bien que se le observe en su quehacer cotidiano $^{187}$.

\section{ANÁLISIS ESTADÍSTICO}

Se realiza en primer lugar un análisis estadístico univariable descriptivo de todas las variables independientes seleccionadas; seguido de un análisis bivariable de correlación de las variables independientes con la variable "acceso a la rehabilitación integral", a través de la prueba Chi-cuadrado de Pearson.

En el primer estudio, el análisis de datos ha sido inicialmente de tipo descriptivo y correlacional bivariable, mediante test chi-cuadrado para valores categóricos y tests tstudent y MWU para valores numéricos. En segundo lugar, se ha realizado un análisis 
multivariable mediante regresión logística, a fin de eliminar posibles variables confusoras. Teniendo en cuenta que el estado civil y el apoyo familiar podían ser variables confusoras asociadas a la edad y lugar de residencia, se ha realizado una regresión logística, tanto de manera individual como junto con el resto de variables independientes, a fin de ajustar los resultados y poder determinar qué factores son realmente explicativos del acceso a la rehabilitación integral.

El análisis estadístico se ha realizado en el primer estudio con el software Python 3.7, utilizando las librerías Matplotlib ${ }^{189}$, Pandas $^{190}$, Statsmodels ${ }^{191}$, Scikit-Learn ${ }^{192}$ y Jamovi ${ }^{193}$.

En el segundo estudio las variables fueron descritas empleando media y desviación estándar o mediana y su rango intercuartílico, según lo adecuado en función de las características de la variable. Las cualitativas se presentan según su distribución de frecuencias. Mediante test Chi-cuadrado de Pearson se analizó asociación de variables cualitativas. En el caso de que el número de celdas con valores esperados menores de 5 es mayor de un 20\%, se ha utilizado el test exacto de Fisher o el test Razón de verosimilitud para variables con más de dos categorías. Las comparaciones de los valores cuantitativos se han realizado mediante la prueba $\mathrm{T}$ de Student para muestras independientes o la prueba U de Mann Whitney.

Se ha realizado análisis univariante de regresión logística para cada una de las variables del estudio asociadas a la rehabilitación integral y pronóstico funcional favorable definido por $\mathrm{BI}>60$. Aquellas que han resultado ser estadísticamente significativas a nivel 0,1 han sido introducidas en un modelo de regresión logística multivariable.

Se utilizó el programa estadístico IBM SPSS Statistics versión 24.0 para Windows. Son considerados estadísticamente significativos valores de $\mathrm{p}<0,05$. 


\section{Capítulo 5.}

RESULTADOS 
Los resultados de la tesis han publicado en dos revistas indexadas, de Salud Pública y Neurología respectivamente, disponibles en el primer anexo.

\section{ANÁLISIS DESCRIPTIVO DE LA MUESTRA DE ESTUDIO}

1.1. Primer estudio: Análisis de los factores asociados a la recuperación integral de pacientes de ictus al alta de Hospital de Agudos

Los datos de las variables descriptivas generales del primer estudio son las siguientes:

Tabla 9. Variables descriptivas (Primer estudio)

\begin{tabular}{|c|c|c|}
\hline $\begin{array}{l}\text { VARIABLES } \\
\text { BASALES }\end{array}$ & Frecuencia & Porcentaje \\
\hline \multicolumn{3}{|l|}{ EDAD } \\
\hline Menor 60 años & 20 & $19 \%$ \\
\hline 61-90 años & 76 & $75 \%$ \\
\hline Mayor 90 años & 6 & $6 \%$ \\
\hline \multicolumn{3}{|l|}{ SEXO } \\
\hline Hombre & 55 & $54 \%$ \\
\hline Mujer & 47 & $46 \%$ \\
\hline \multicolumn{3}{|c|}{ ESTADO CIVIL } \\
\hline Casada & 56 & $55 \%$ \\
\hline Viuda & 28 & $27 \%$ \\
\hline Soltera & 18 & $18 \%$ \\
\hline \multicolumn{3}{|c|}{ LUGAR DE RESIDENCIA } \\
\hline Urbana & 58 & $56 \%$ \\
\hline Rural & 44 & $44 \%$ \\
\hline \multicolumn{3}{|l|}{ ECONOMIA } \\
\hline Inferior $500 €$ & 21 & $20,6 \%$ \\
\hline $500-1000 €$ & 33 & $32,4 \%$ \\
\hline Mas $1000 €$ & 48 & $47 \%$ \\
\hline \multicolumn{3}{|c|}{ APOYO FAMILIAR } \\
\hline Si tiene apoyo & 60 & $59 \%$ \\
\hline No tiene apoyo & 42 & $41 \%$ \\
\hline
\end{tabular}

Fuente: Elaboración propia 
1.2. Segundo Estudio: Estudio del impacto de la RHB integral sobre la recuperación funcional a largo plazo de los pacientes con ictus

\subsubsection{Variables descriptivas:}

Se presenta las variables descriptivas de la muestra en la tabla 10 .

Tabla 10. Variables descriptivas (Segundo Estudio)

\begin{tabular}{|l|l|l|}
\hline VARIABLES BASALES & Frecuencia & Porcentaje \\
\hline
\end{tabular}

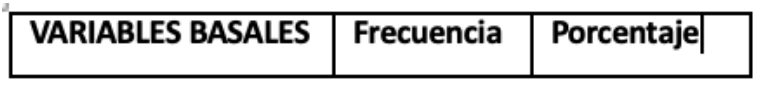

\begin{tabular}{|c|c|c|}
\hline \multicolumn{3}{|l|}{ EDAD } \\
\hline Menor 60 años & 40 & $23,4 \%$ \\
\hline 61-80 años & 80 & $46,8 \%$ \\
\hline Mayor 80 años & 51 & $29,8 \%$ \\
\hline \multicolumn{3}{|l|}{ SEXO } \\
\hline Hombre & 87 & $50,9 \%$ \\
\hline Mujeres & 84 & $49,1 \%$ \\
\hline \multicolumn{3}{|l|}{ ESTADO CIVIL } \\
\hline Casada & 93 & $54,4 \%$ \\
\hline Viuda & 34 & $19,9 \%$ \\
\hline Soltera & 32 & $18,7 \%$ \\
\hline Separada & 12 & $7 \%$ \\
\hline \multicolumn{3}{|c|}{ ACTIVIDAD LABORAL } \\
\hline Jubilado & 62 & $36,3 \%$ \\
\hline No jubilado & 109 & \\
\hline \multicolumn{3}{|c|}{ ECONOMIA FAMILIAR } \\
\hline$>1350 €$ & 139 & $81,3 \%$ \\
\hline $1351-2000 €$ & 23 & $13,5 \%$ \\
\hline$>2000 €$ & 9 & $5,3 \%$ \\
\hline \multicolumn{3}{|c|}{ LUGAR DE RESIDENCIA } \\
\hline Urbano & 121 & $70,8 \%$ \\
\hline Rural & 50 & $29,2 \%$ \\
\hline \multicolumn{3}{|c|}{ ACCESIBILIDAD VIVIENDA } \\
\hline Adecuada & 87 & $50,9 \%$ \\
\hline Con escaleras & 34 & $29,2 \%$ \\
\hline Barreras & 50 & $19,9 \%$ \\
\hline
\end{tabular}

\begin{tabular}{|l|l|l|}
\hline \multicolumn{2}{|l|}{ PROPIEDAD VIVIENDA } \\
\hline Vivienda propia & 132 & $77,2 \%$ \\
\hline No propia & 39 & $22,8 \%$ \\
\hline REGIMEN VIVIENDA & \multicolumn{2}{|l|}{} \\
\hline Propiedad & 132 & $77,2 \%$ \\
\hline Alquiler & 24 & $14 \%$ \\
\hline Cedida al uso & 15 & $8,8 \%$ \\
\hline CONVIVIENTES & & \\
\hline
\end{tabular}

\begin{tabular}{|l|l|l|}
\hline Vive solo & 60 & $35,1 \%$ \\
\hline Viven 2 personas & 87 & $50,9 \%$ \\
\hline Viven 3 o más personas & 24 & $14 \%$ \\
\hline APOYO FAMILIAR & 109 & $63,7 \%$ \\
\hline Si tiene apoyo & 62 & $36,3 \%$ \\
\hline No tiene apoyo & \multicolumn{2}{|l|}{} \\
\hline RIESGO SOCIAL AL INGRESO & $55,6 \%$ \\
\hline SI Riesgo Social & 95 & $44,4 \%$ \\
\hline NO Riesgo SOcial & 76 & $1,8 \%$ \\
\hline APOYOS SOCIALES & 3 & $98,2 \%$ \\
\hline SI Apoyo Social & 168 & $9,5 \%$ \\
\hline No Apoyo Social & 162 & $96,5 \%$ \\
\hline INTERVENCION SOCIAL ANTERIOR \\
\hline NO & 162 & $5,3 \%$ \\
\hline SI & 9 & \\
\hline RECONOCIMIENTO LPAPAD INGRESO \\
\hline En trámite & 6 & \\
\hline No solicitada & 165 & \\
\hline
\end{tabular}

Fuente: Elaboración propia 


\subsubsection{Factores de riesgo}

Los factores de riesgo cerebrovascular que presentó nuestra población objeto de estudio se detallan en el siguiente Gráfico 1. La hipertensión arterial fue el factor de riesgo más frecuente $(53,8 \%)$, seguido de diabetes $(24 \%)$, bebedor de riesgo $(20,5 \%)$ y tabaquismo $(17 \%)$. Otros factores de riesgo cerebrovasculares que aparecieron en nuestra población objeto de estudio fueron: ansiedad (17,5\%), depresión (17\%), insomnio (9,9\%).

\section{Gráfico 1. Factores de riesgo cerebrovascular}

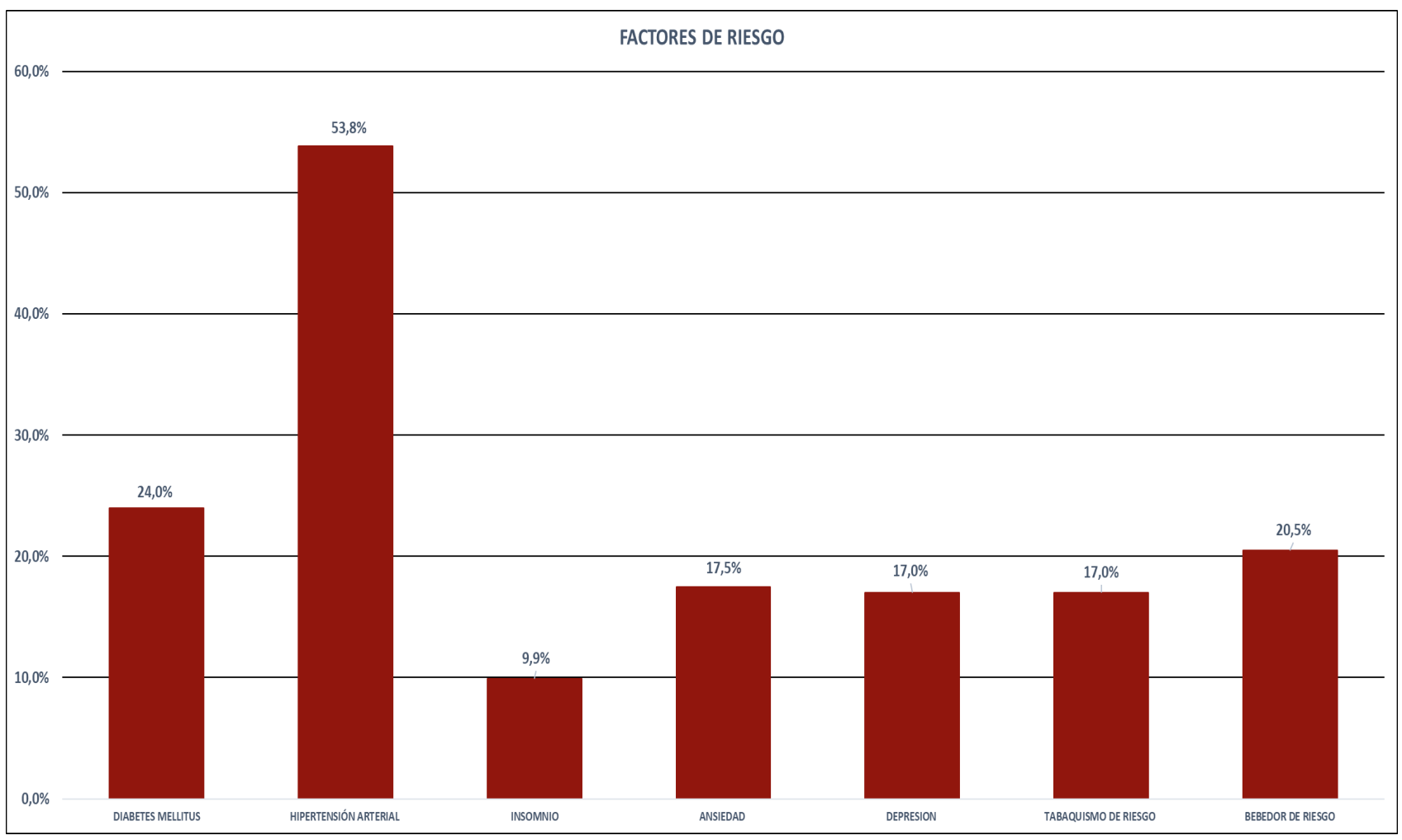

Fuente: Elaboración propia

\subsubsection{Gravedad inicial del ictus}

La gravedad del ictus al ingreso hospitalario se refleja en la tabla 11.

Tabla 11. NIHSS al ingreso hospitalario

\begin{tabular}{|l|l|l|}
\hline NIHSS & N & Porcentaje \\
\hline$<4$ & 57 & $33,3 \%$ \\
\hline $4-15$ & 90 & $52,6 \%$ \\
\hline$\geq 16$ & 19 & $11,1 \%$ \\
\hline Perdidos & 5 & $2,9 \%$ \\
\hline
\end{tabular}

La mediana de NIHSS al ingreso fue de 5 (Rango intercuartílico 2-9). 


\subsubsection{Rankin previo al ictus}

Al ingreso hospitalario del paciente, se comprobó el Rankin que tenía antes del ingreso. Se confirmó que Rankin $\leq 2$ tenían 160 (94\%) personas, 7 antes del ingreso se encontraban en un Rankin 3 (4\%) y 4 (2\%) personas antes del ingreso se encontraban en un Rankin 4.

\section{Gráfico 2. Rankin previo al ictus}

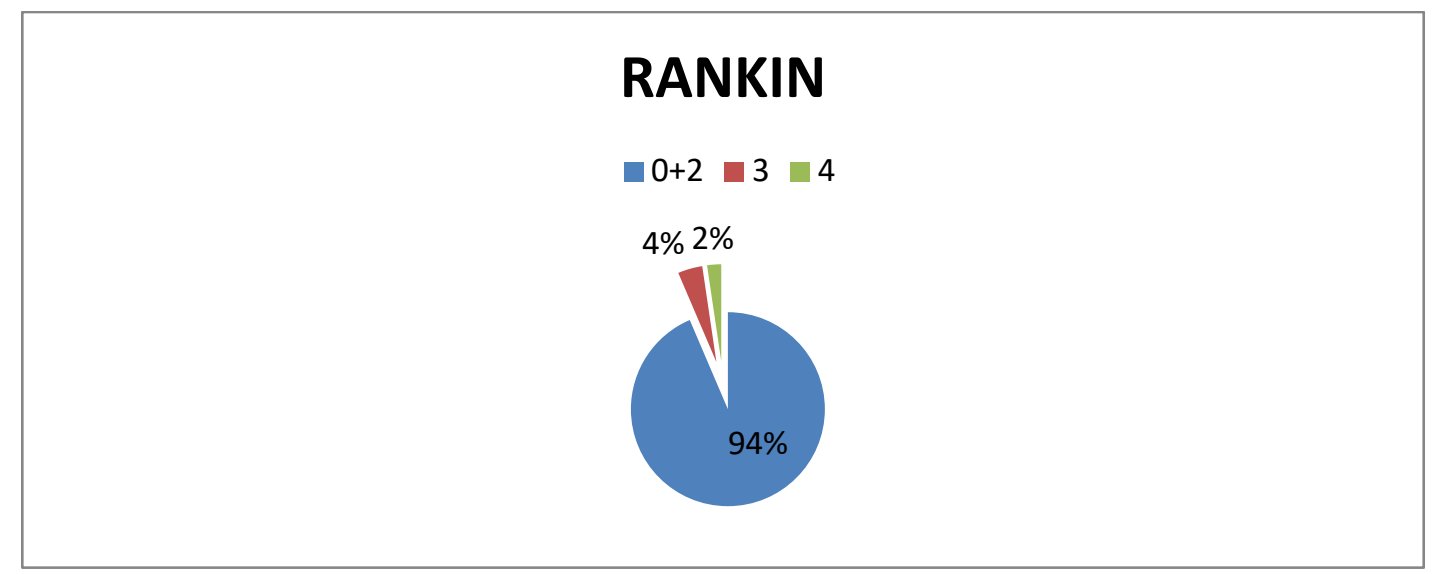

Fuente: Elaboración propia

\subsubsection{Evolución clínica al Alta hospitalaria}

Al alta hospitalaria se comprobó:

A/Gravedad alta hospitalaria.

Se refleja la gravedad en la siguiente tabla

Tabla 12. NIHSS al Alta

\begin{tabular}{|l|l|l|}
\hline NIHSS & N & PORCENTAJE \\
\hline$<4$ & 81 & $47,4 \%$ \\
\hline $4-15$ & 75 & $43,9 \%$ \\
\hline$\geq 16$ & 5 & $2,9 \%$ \\
\hline Perdidos & 10 & $5,8 \%$ \\
\hline
\end{tabular}

La mediana de NIHSS al alta hospitalaria fue de 3 (Rango intercuartílico 2-7).

B/Rankin alta hospitalaria

El Rankin al alta hospitalaria se refleja en el siguiente Gráfico 3.

El 58,5\% Rankin $\leq 2$, el 14\% Rankin 3, el 15,2 Rankin 4 y el 12,3\% Rankin 5. 
Gráfico 3. Rankin al alta hospitalaria

\section{RANKIN ALTA HOSPITALARIA}

$$
\square 0+2 \backsim 3 \backsim 4 \backsim 5
$$

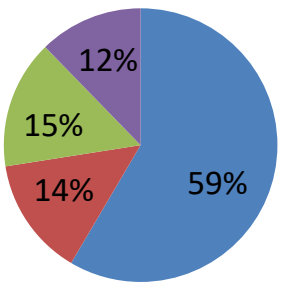

Fuente: Elaboración propia

\subsubsection{Tratamiento rehabilitador que precisan al alta.}

Al alta hospitalaria se registro el tratamiento rehabilitador que precisaba nuestra población objeto de estudio, destacó la fisioterapia (83\%), seguida de la atención personal $(69,6 \%)$, la logopedia $(64,9 \%)$, terapia ocupacional $(53,8 \%)$, neuropsicologo $(39,2 \%)$, salud mental $(30,4 \%)$ y rehabilitación visual $(21,1 \%)$. Se muestra en el siguiente Gráfico.

\section{Gráfico 4. Porcentaje tratamiento rehabilitador precisan}

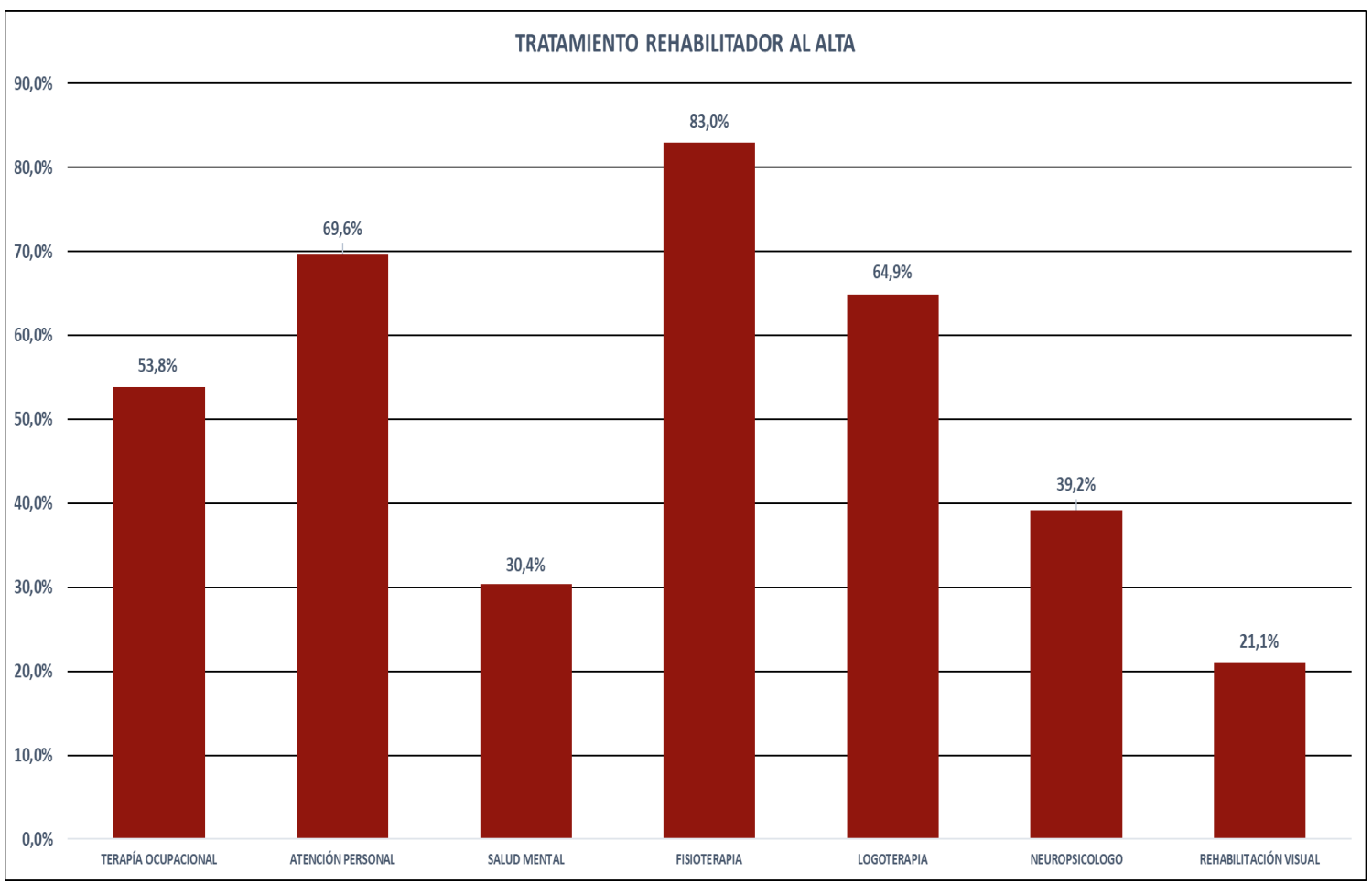

Fuente: Elaboración propia 


\section{A/ Seguimiento a los 3 meses}

A los 3 meses se comprobó si estaba recibiendo el tratamiento rehabilitador y la modalidad de tratamiento.

En relación a la modalidad de tratamiento rehabilitador que estaba recibiendo. Se muestra en el siguiente Gráfico 5. La población objeto de nuestro estudio recibió al alta la rehabilitación en centro concertado (12,9\%), centro público $(10,5 \%)$, tratamiento público + privado $(46,2 \%)$, privado $(29,8 \%)$ y no recibió nada $(6 \%)$. Se refleja en el Gráfico 5 :

Gráfico 5. Modalidad de rehabilitación

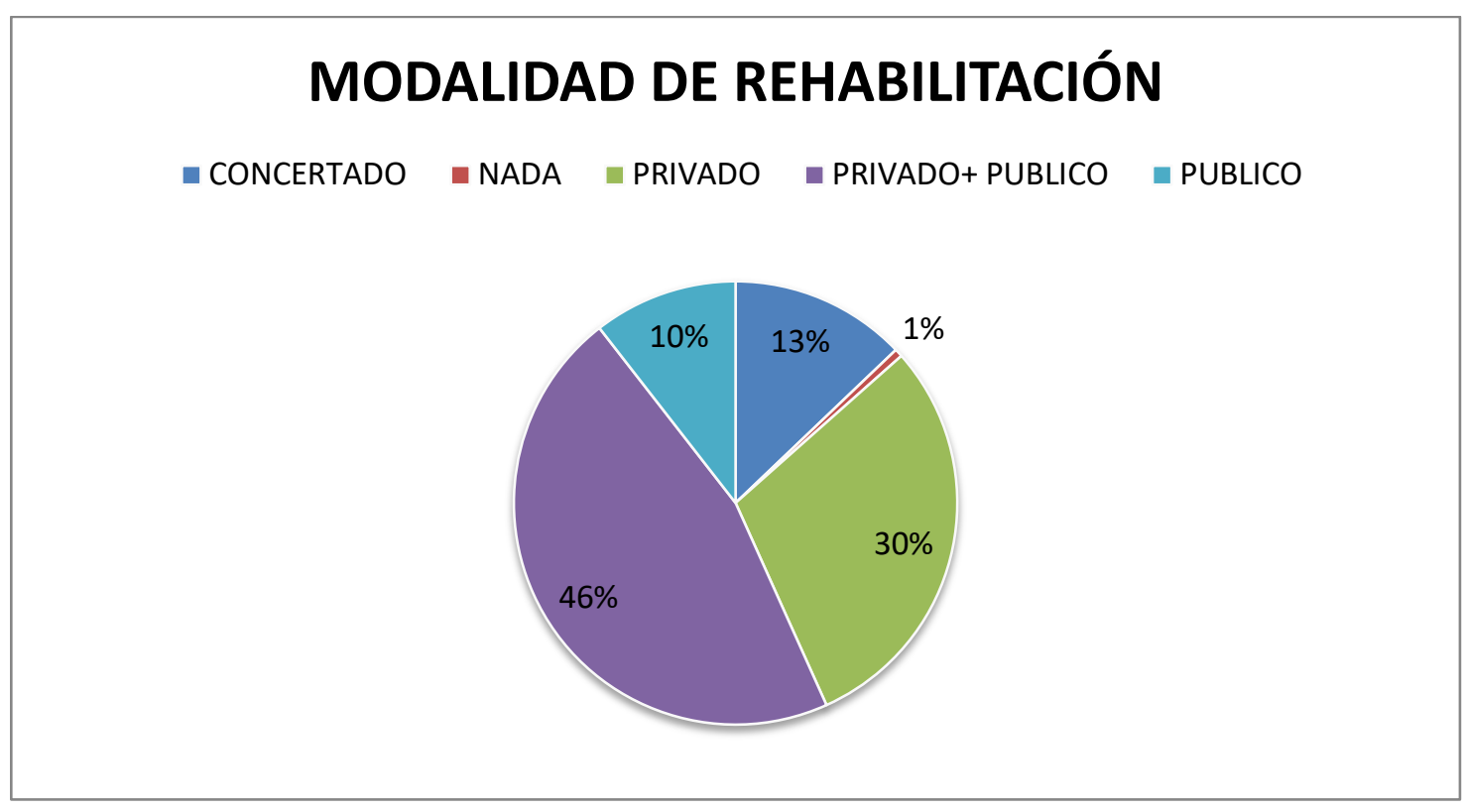

Fuente: Elaboración propia

\section{RESULTADOS VARIABLES ASOCIADAS A REHABILITACIÓN INTEGRAL}

\subsection{Primer Estudio: Análisis de los factores asociados a la recuperación integral de} pacientes de ictus al alta de Hospital de Agudos

E1 53,9\% ha recibido rehabilitación integral, es decir, el tratamiento requerido según todas las áreas afectadas tanto desde servicios públicos (fisioterapia, logopedia y atención social) como a nivel privado (neuropsicología, terapia ocupacional). El 46,1\% restante, a pesar de ser susceptible de rehabilitación integral, no ha podido acceder a la misma. Las variables basales asociadas al acceso a la RHBI se muestran en la siguiente tabla. 
Tabla 13. Rehabilitación según variables basales $(n=102)$ (Primer estudio)

\begin{tabular}{|c|c|c|c|}
\hline VARIABLES BASALES & $\begin{array}{l}\text { Rehabilitación } \\
\text { SI }\end{array}$ & $\begin{array}{l}\text { Rehabilitación } \\
\text { NO }\end{array}$ & $\begin{array}{l}\text { P-valor (Chi- } \\
\text { cuadrado) }\end{array}$ \\
\hline - RESIDENCIA URBANA* & $39(38 \%)$ & $18(18 \%)$ & $<0.001$ \\
\hline - RESIDENCIA RURAL* & $16(16 \%)$ & $29(28 \%)$ & \\
\hline - SEXO: MASCULINO & $33(32 \%)$ & $22(22 \%)$ & 0.18 \\
\hline - SEXO: FEMENINO & $22(22 \%)$ & $25(25 \%)$ & \\
\hline - CASADO* & $32(31 \%)$ & $24(24 \%)$ & \\
\hline - SOLTERO* & $14(14 \%)$ & $4(4 \%)$ & 0.007 \\
\hline - VIUDO* & $9(9 \%)$ & $19(19 \%)$ & \\
\hline - EDAD (media)* & 66,2 & 81,6 & $<0.001$ \\
\hline -INGRESOS MENSUALES (media) & $856 €$ & $901 €$ & 0.62 \\
\hline - APOYO FAMILIAR (SI) * & $26(25 \%)$ & $34(33 \%)$ & 0.01 \\
\hline - APOYO FAMILIAR (NO)* & $29(28 \%)$ & $13(13 \%)$ & \\
\hline
\end{tabular}

* Variables con una asociación significativa en el test Chi-Cuadrado

Como se muestra en la tabla 13, fueron cuatro los factores asociados al acceso a la rehabilitación integral: el lugar de residencia (medio rural/medio urbano), el estado civil, la edad y la existencia de apoyo familiar. Otros factores, como los ingresos económicos o el sexo, no presentaron asociación significativa.

El estado civil fue el tercer factor que presentó diferencias significativas en el acceso a la rehabilitación integral $(\mathrm{p}=0,008)$. Ahora bien, analizando conjuntamente la edad y el estado civil (figura 1), y considerando que la edad media de las personas viudas era de 81,4 años, la de las casadas de 74,4 años y la de las solteras de 57,3 años, para determinar si el estado civil podría ser un factor de confusión al estar en sí mismo correlacionado con la edad, se realizó tanto un test ANOVA como un Kruskal-Wallis, para evaluar la diferencia de medias de edad entre los tres grupos. Ambos fueron significativos $(\mathrm{p}<0,0001)$, por lo que podemos concluir que era un factor de confusión altamente 
correlacionado con la edad (figura 1).

Figura 1. Distribución del acceso a rehabilitación integral por edad y estado civil

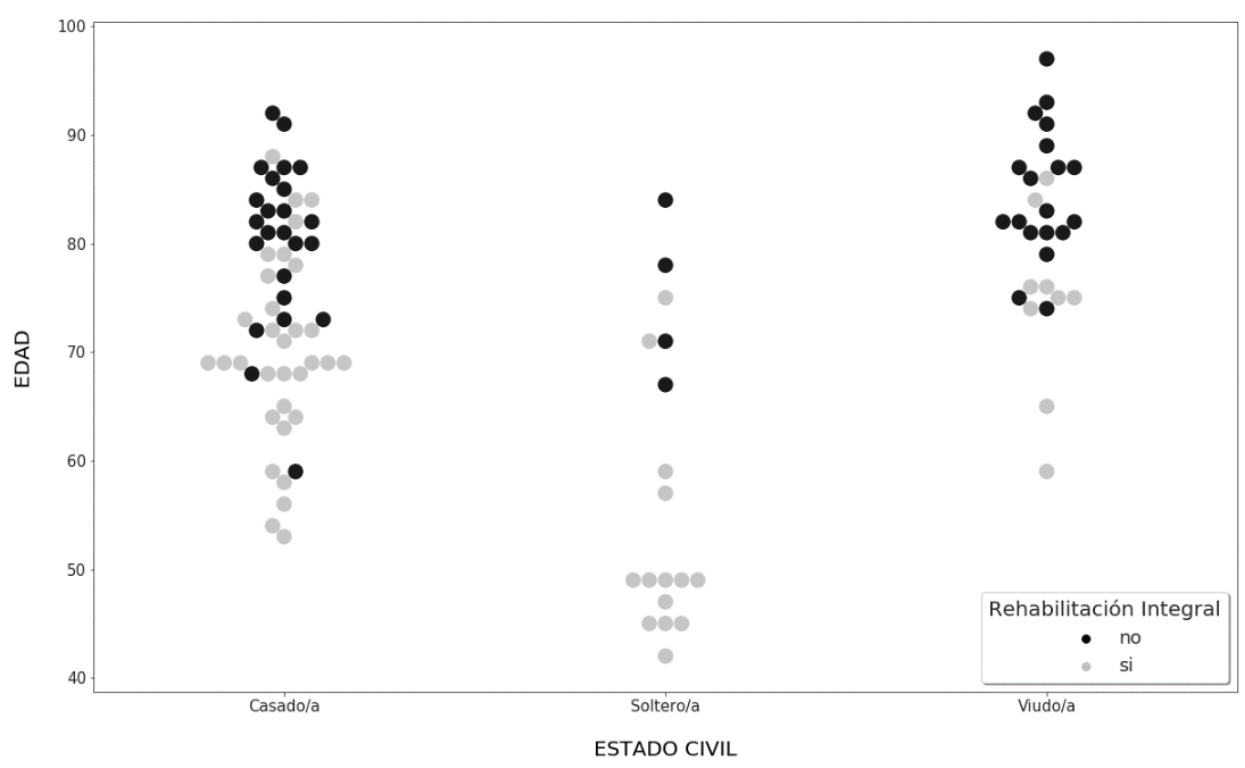

Un cuarto factor asociado al acceso a la rehabilitación integral fue el apoyo familiar $(\mathrm{p}=0.018)$.

Cuando estas variables fueron llevadas a un modelo de regresión logística multivariable, resultaron variables asociadas al acceso a RHB integral las que se muestran en la Tabla 14.

Tabla 14. Variables explicativas del acceso a la rehabilitación integral

\begin{tabular}{|lll|}
\hline Variable & $\begin{array}{l}\text { Coeficiente }(\mathbf{P}-\text { Valor) } \\
\text { (Variable individual) }\end{array}$ & $\begin{array}{l}\text { Coeficiente / OR (P-valor) } \\
\text { (Ajustado al resto de variables) }\end{array}$ \\
EDAD & $-0.17(<0.001)$ & $-0.226 / 0.8(<0.001)$ \\
LOCALIDAD (urbana) & $1.37(0.001)$ & $2.85 / 17.4(<0.001)$ \\
APOYO FAMILIAR (si) & $1.1(0.011)$ & $-0.21 / 0.81(0.77)$ \\
CASADO (si) & $0.29(0.47)$ & $-0.35 / 1.42(0.64)$ \\
\hline
\end{tabular}

Se compararon las medias de edad entre ambas localidades con un test T de Student y no hubo diferencias significativas en la edad media de los pacientes de localidad rural y urbana $(p=0,7)$, por lo que ambos factores eran independientes entre sí. Tanto la edad como el tipo de localidad de residencia evidenciaron ser los factores claramente explicativos del acceso a la rehabilitación integral. En los análisis se observó que el resto de variables independientes actuaron como factores de confusión, quedando demostrado en los análisis iniciales y finalmente comprobados en la regresión logística multivariable Atendiendo al resultado del análisis de regresión, la asociación que presenta el estado 
civil está fuertemente condicionada por la edad, por lo que el estado civil no puede ser considerado como factor asociado, al menos en la muestra de nuestro estudio.

En relación al apoyo familiar que se mostro está mucho más presente en la zona rural que en la urbana. Esta circunstancia confirma que la variable "apoyo familiar" es un factor de confusión en el análisis de regresión

\subsection{Segundo Estudio: Estudio del impacto de la RHB integral sobre la recuperación funcional a largo plazo de los pacientes con ictus}

Solo el 53,2\% de nuestra población objeto de estudio recibió la RHB integral, frente al $46,8 \%$ que no accedió. El 76\% tuvo que pagar servicios privados para tener acceso a la rehabilitación integral.

La relación entre las variables basales y el acceso a una rehabilitación integral tras el alta hospitalaria aparecen en la Tabla 17. Las variables asociadas en el modelo univariante fueron:

Tabla 15. Relación entre variables basales y acceso rehabilitación integral $(n=171)$

\begin{tabular}{|c|c|c|c|}
\hline Variables & RHB Integral: Si & RHB Integral: No & P-valor \\
\hline MUESTRA & 91 personas & 80 personas & \\
\hline \multicolumn{4}{|l|}{ SEXO } \\
\hline Hombre & $52(57,1 \%)$ & $35(43,8 \%)$ & 0,080 \\
\hline Mujer & $39(42,95 \%)$ & $45(56,3 \%)$ & \\
\hline \multicolumn{4}{|l|}{ EDAD } \\
\hline$\geq 81$ años & $21(23,1 \%)$ & $30(37,5 \%)$ & 0,001 \\
\hline 61-80 años & $39(42,9 \%)$ & $41(51,3 \%)$ & \\
\hline$\leq 60$ años & $31(34,1 \%)$ & $9(11,3 \%)$ & \\
\hline EDAD (media) & 65,23 & 81,6 & \\
\hline \multicolumn{4}{|l|}{ ESTADO CIVIL } \\
\hline Casado & $48(51,6 \%)$ & $45(48,4 \%)$ & \\
\hline Divorciado & $6(50 \%)$ & $6(50 \%)$ & 0,086 \\
\hline Soltero & $23(71,9 \%)$ & $9(28,1 \%)$ & \\
\hline Viudo & $14(41,2 \%)$ & $20(58,8 \%)$ & \\
\hline \multicolumn{4}{|l|}{ CONVIVIENTES } \\
\hline Solo & $36(39,6 \%)$ & $24(30 \%)$ & 0,026 \\
\hline 2 personas & $38(41,8 \%)$ & $49(61,3 \%)$ & \\
\hline 3 o más personas & $17(18,7 \%)$ & $7(8,8 \%)$ & \\
\hline \multicolumn{4}{|c|}{ ACTIVIDAD LABORAL } \\
\hline Jubilado & $34(37,4 \%)$ & $28(35 \%)$ & 0,748 \\
\hline No jubilado & $57(62,6 \%)$ & $52(65 \%)$ & \\
\hline
\end{tabular}




\begin{tabular}{|c|c|c|c|}
\hline Variables & RHB Integral: Si & RHB Integral: No & P-valor \\
\hline ECONOMIA (MEDIA) & $1197,7422 €$ & $1139,9375 €$ & 0,335 \\
\hline \multicolumn{4}{|l|}{ RESIDENCIA } \\
\hline Rural & $30(37,5 \%)$ & $20(22 \%)$ & 0,026 \\
\hline Urbana & $50(62,5 \%)$ & $71(78 \%)$ & \\
\hline \multicolumn{4}{|l|}{ ACCESIBILIDAD } \\
\hline \multicolumn{4}{|l|}{ VIVIENDA } \\
\hline Adecuada & $56(61,5 \%$ & $31(38,8 \%)$ & 0,003 \\
\hline No adecuada & $35(38,5 \%)$ & $49(61,35 \%)$ & \\
\hline \multicolumn{4}{|l|}{ PROPIEDAD } \\
\hline \multicolumn{4}{|l|}{ VIVIENDA } \\
\hline Si & $63(69,2 \%)$ & $69(86,3 \%)$ & 0,008 \\
\hline No & $28(30,8 \%)$ & $11(13,8 \%)$ & \\
\hline \multicolumn{4}{|l|}{ RIESGO SOCIAL } \\
\hline \multicolumn{4}{|l|}{ INGRESO } \\
\hline $\mathrm{Si}$ & $50(54,9 \%)$ & $45(56,3 \%)$ & 0,864 \\
\hline No & $41(45,1 \%)$ & $35(43,8 \%)$ & \\
\hline \multicolumn{4}{|l|}{ APOYO FAMILIAR } \\
\hline Si & $62(56,9 \%)$ & $47(43,1 \%)$ & 0,203 \\
\hline No & $29(46,8 \%)$ & $33(53,2 \%)$ & \\
\hline \multicolumn{4}{|l|}{ INTERVENCIÓN } \\
\hline \multicolumn{4}{|l|}{ SOCIAL ANTERIOR } \\
\hline Si & $5(55,6 \%)$ & $4(44,4 \%)$ & 1,000 \\
\hline No & $86(53,1 \%)$ & $76(46,9 \%)$ & \\
\hline \multicolumn{4}{|l|}{ RECONOCIMIENTO } \\
\hline \multicolumn{4}{|l|}{ LPAPAD } \\
\hline En trámite & $3(3,3 \%)$ & $3(3,8 \%)$ & 1,000 \\
\hline No & $88(96,7 \%)$ & $77(96,3 \%)$ & \\
\hline \multicolumn{4}{|l|}{ APOYO SOCIAL } \\
\hline $\mathrm{Si}$ & $88(96,7 \%)$ & 80 (100\%) & 1,000 \\
\hline No & $3(3,3 \%)$ & $0(0,0 \%)$ & \\
\hline \multicolumn{4}{|l|}{ RANKIN ALTA ${ }^{a}$} \\
\hline$\leq 2$ & $60(65,9 \%)$ & $40(50 \%)$ & 0,019 \\
\hline 3 & $14(15,4 \%)$ & $10(12,5 \%)$ & \\
\hline 4 & $12(13,2 \%)$ & $14(17,5 \%)$ & \\
\hline 5 & $5(5,5 \%)$ & $16(20 \%)$ & \\
\hline NIHSS ALTA MEDIA & 7,08 & 6,51 & 0,442 \\
\hline \multicolumn{4}{|l|}{ MODALIDAD } \\
\hline \multicolumn{4}{|l|}{ PRIVADA } \\
\hline Si & $62(68,1 \%)$ & $68(85 \%)$ & 0,010 \\
\hline No & $29(31,9 \%)$ & $12(15 \%)$ & \\
\hline \multicolumn{4}{|l|}{ DIABETES } \\
\hline Si & $16(17,6 \%)$ & $25(31,3 \%)$ & 0,037 \\
\hline No & $75(82,4 \%)$ & $55(68,8 \%)$ & \\
\hline
\end{tabular}




\begin{tabular}{|c|c|c|c|}
\hline Variables & RHB Integral: Si & RHB Integral: No & P-valor \\
\hline \multicolumn{4}{|l|}{ HIPERTENSIÓN } \\
\hline \multicolumn{4}{|l|}{ ARTERIAL } \\
\hline $\mathrm{Si}$ & $44(48,4 \%)$ & $48(60 \%)$ & 0,127 \\
\hline No & $47(51,6 \%)$ & $32(40 \%)$ & \\
\hline DISTANCIA (MEDIA) & $85,023 \mathrm{kms}$ & $14,68 \mathrm{kms}$ & 0,002 \\
\hline
\end{tabular}

Cuando estas variables fueron llevadas a un modelo de regresión logística multivariable, resultaron variables asociadas al acceso a RHB integral las mostradas en Tabla 16.

Tabla 16. Modelo de regresión logística multivariable para factores predictores de acceso a rehabilitación integral

\begin{tabular}{|c|c|c|c|c|}
\hline & \multirow[t]{2}{*}{ Odds ratio } & \multicolumn{2}{|c|}{ 95\% C.I para OR } & \multirow[t]{2}{*}{ P-valor } \\
\hline & & Inferior & Superior & \\
\hline \multicolumn{5}{|l|}{ Edad } \\
\hline$\geq 81$ años & & & & 0,438 \\
\hline 61-80 años & 0,715 & 0,175 & 2,924 & \\
\hline$\leq 60$ años & 0,704 & 0,290 & 1,709 & \\
\hline \multicolumn{5}{|l|}{ Convivientes } \\
\hline 1 persona & 2,290 & 0,967 & 5,420 & 0,060 \\
\hline \multicolumn{5}{|l|}{2 personas (referencia) } \\
\hline 3 o más personas & 1,888 & 0,532 & 6,701 & \\
\hline $\begin{array}{l}\text { Lugar residencia } \\
\text { (Urbano vs Rural) }\end{array}$ & 2,957 & 1,067 & 8,199 & 0,037 \\
\hline $\begin{array}{l}\text { Accesibilidad Vivienda } \\
\text { (Si vs No) }\end{array}$ & 1,810 & 0,780 & 4,200 & 0,167 \\
\hline $\begin{array}{l}\text { Propiedad vivienda ( } \mathrm{Si} \\
\text { vs No) }\end{array}$ & 1,357 & 0,446 & 4,129 & 0,590 \\
\hline \multicolumn{5}{|l|}{ Rankin alta } \\
\hline$\leq 2$ & 22,437 & 3,247 & 155,058 & 0,014 \\
\hline 3 & 14,014 & 1,666 & 117,884 & \\
\hline 4 & 10,786 & 1,363 & 85,531 & \\
\hline \multicolumn{5}{|l|}{5} \\
\hline Modalidad privada & 2,890 & 1,130 & 7,392 & 0,027 \\
\hline Distancia (Media) & 1,012 & 0,998 & 1,026 & 0,090 \\
\hline
\end{tabular}




\section{IMPACTO DE LA RHB INTEGRAL POST-ICTUS SOBRE EL PRONÓSTICO FUNCIONAL Y SITUACIÓN A LARGO PLAZO DE LOS PACIENTES DEL ESTUDIO (5 AÑOS)}

En febrero-marzo 2020 de los 171 pacientes de la muestra inicial, el 19,9\% (n:34) habían fallecido y el 80,1\% (n:137) seguían vivos. Estos datos se han comprobado a lo largo del estudio a través de su historia clínica, el Registro Civil y contacto con los familiares.

Por años, en el año 2015 fallecieron 6 pacientes, en el año 2016 fallecieron 14 pacientes, posteriormente en el año 2017 fallecieron 5 pacientes, en el año 2018 han fallecido 4 pacientes y en el año 2019 han fallecido 5 pacientes.

Según su edad, estos 34 exitus tenían una edad que se corresponde:

El 64,7\% (n:22) de los fallecidos tenía más de 80 años, el 32,4\% (n:11) tenía entre 61-80 años y el 2,9\% (n:1) tenía menos de 60 años. Se muestra en el siguiente Gráfico 6:

Gráfico 6. Exitus a marzo 2020

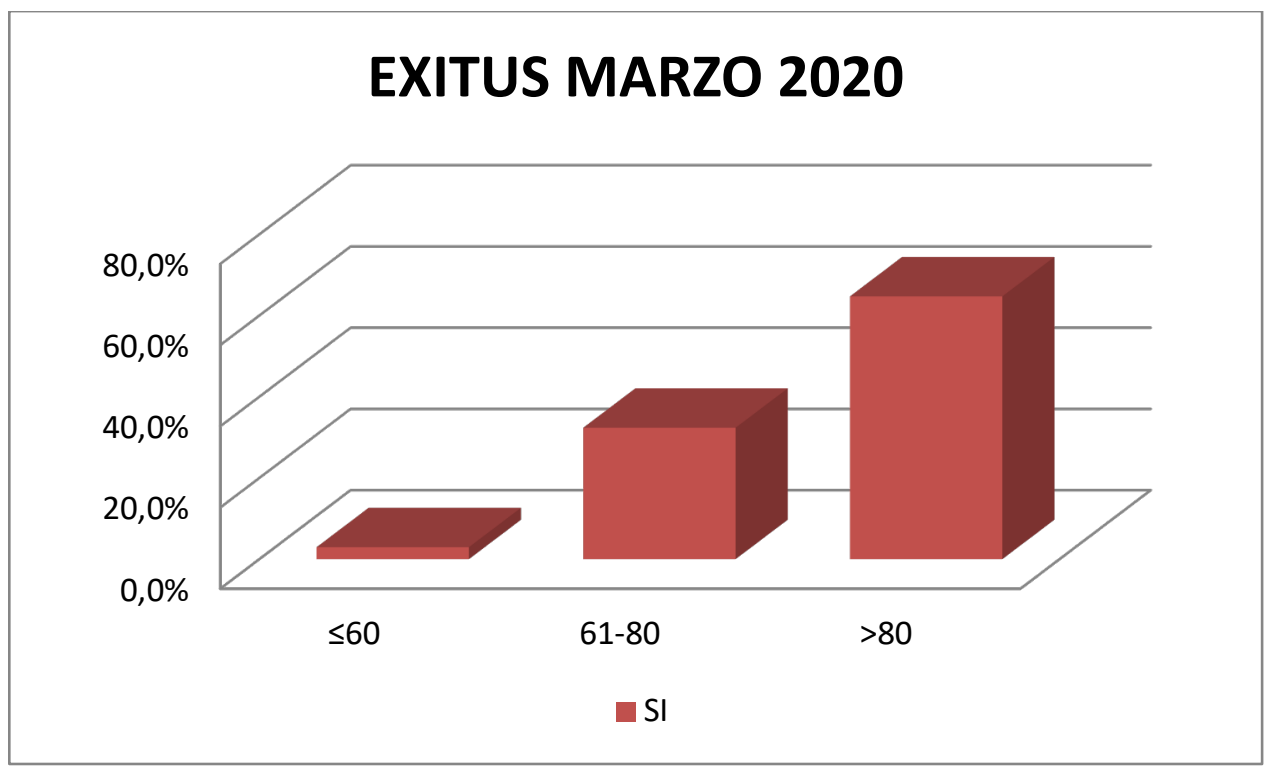

Fuente: Elaboración propia

\subsection{Acceso a RHB Integral post-ictus}

Cómo se refleja en la Gráfica 7, en relación a los 137 supervivientes, acceden a RHB integral el 56,2\% frente al 43\% que no accedió. 
Gráfico 7. Acceso a RHBI.

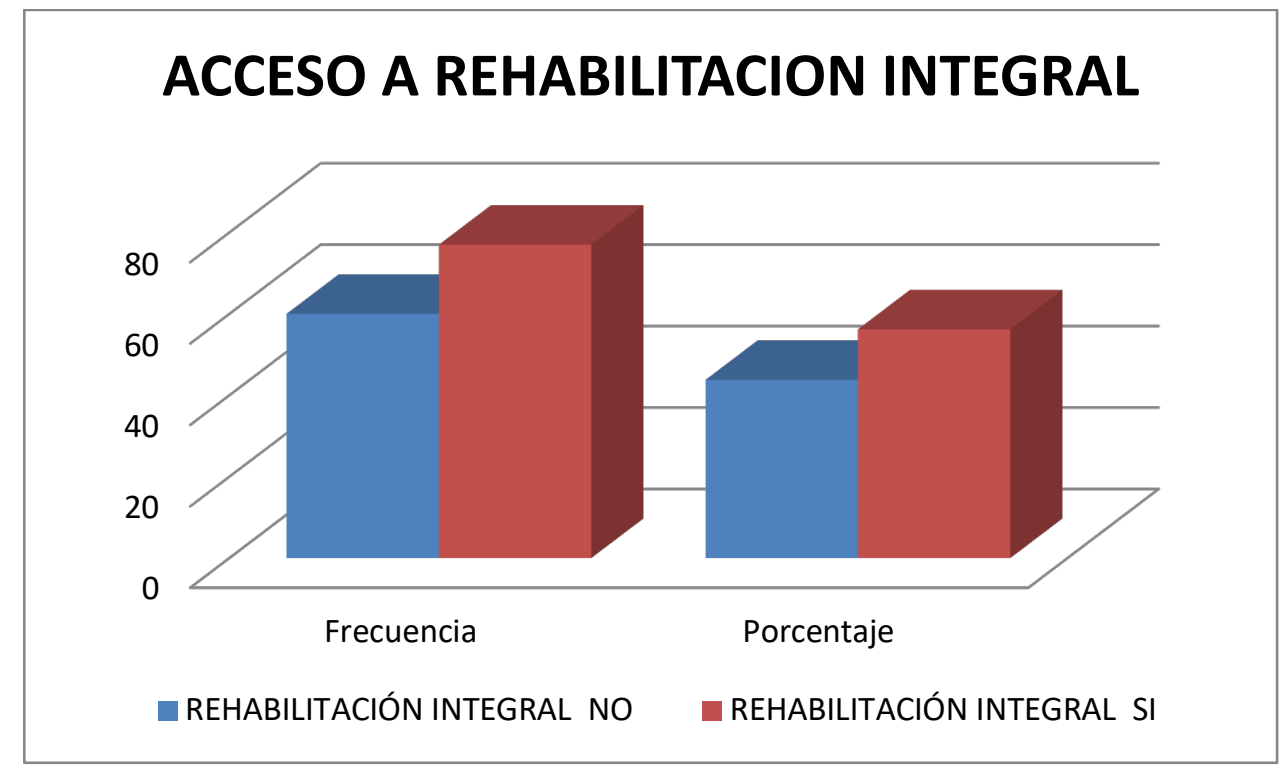

Fuente: Elaboración propia

Los que no acceden (60 personas) una economía media de 1081,25€ y los que si acceden (77 personas) una economía media de 1224,32€. Las variables asociadas al acceso en este segundo estudio se comentaron en el apartado anterior.

\subsection{Evolución funcional}

Se analizó el efecto a largo plazo de la RHB integral en la autonomía funcional de pacientes que fueron alta hospitalaria en el año 2015 y sobrevivían en marzo de 2020. Llegaron al final del estudio 137 pacientes, cuya mediana de puntuación en el BI fue de $56,3$ en 76 pacientes $(55,5 \%)$ alcanzando el rango de dependencia leve (BI $>60)$.

La evolución funcional a largo plazo en nuestra población objeto de estudio se valoró a través del Índice Barthel. Se muestra el resultado en el siguiente Gráfico 8. 


\section{Gráfico 8. Evolución temporal del pronóstico funcional y su relación con la RHB}

integral

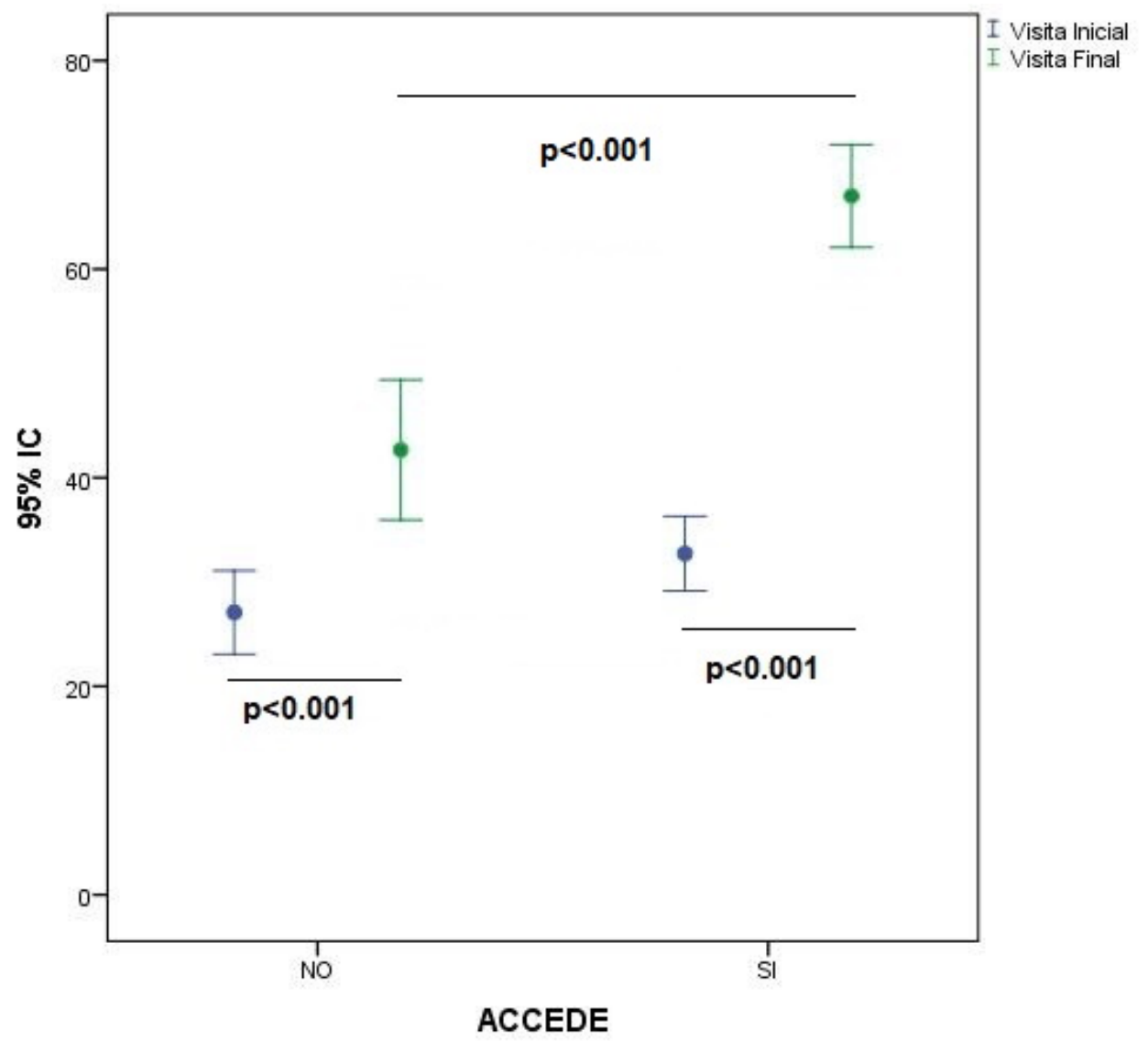

En cuanto a la mejoría en el desempeño de las actividades básicas de la vida diaria se demuestra, a través de la aplicación del Barthel una diferencia media de 25,72 puntos desde el alta en 2015 a marzo 2020 en los que si acceden a la rehabilitación integral frente una media de 15,58 puntos en Barthel desde el alta hospitalaria hasta marzo 2020 en los que no acceden a la rehabilitación integral.

De los pacientes que fueron alta en el año 2015, 77 pacientes $(56,2 \%)$ presentaban dependencia grave $(>20-40)$ y dependencia moderada $(>40-60)$ y 60 pacientes $(43,8 \%)$ dependencia total (0-20). En marzo 2020, los pacientes con dependencia total eran 26 (19\%), con dependencia grave y moderada 35 pacientes $(35,5 \%)$, y con dependencia leve 76 (55,5\%). Transcurrido un período medio de 54 meses desde el inicio del tratamiento rehabilitador integral (período entre 50 y 62 meses, dependiendo de mes de alta hospitalaria durante 2015), se constata una disminución de pacientes con dependencia total, grave y moderada respecto a su situación al alta hospitalaria, estadísticamente significativa $(\mathrm{p}=0,001 \mathrm{y} \mathrm{p}=0,001)$. Es decir, los pacientes con $\mathrm{BI}>60$ puntos a largo plazo aumentan al $55,5 \%$ de la muestra frente al $100 \%$ de pacientes con $\mathrm{BI}<60$ puntos al alta hospitalaria. 
Cuando estas variables fueron llevadas a un modelo de regresión logística multivariante, resultaron como predictores de mejor pronóstico funcional las siguientes: acceso a rehabilitación integral (OR: 12,441, (IC 95\% 4,7-32,5), p<0,001), Rankin al alta (OR: 6,644, (IC 95\% 1,2-36,6), p=0,030) y la menor distancia en kilómetros al centro de rehabilitación (OR: 0,997, (IC 95\% 0,994-0,999), p=0,024). 


\section{Capítulo 6. DISCUSIÓN}




\section{ACCESO DE LOS PACIENTES A REHABILITACIÓN INTEGRAL POST-ICTUS}

El tratamiento rehabilitador se estableció en base a los dominios que pueden verse afectados por el ictus. En el segundo estudio se refleja específicamente el tratamiento rehabilitador que más necesitaron los pacientes, en primer lugar, la fisioterapia, seguido de auxiliar de atención personal para ayudarle en la realización de las actividades básicas de la vida diaria, logopedia, terapia ocupacional, neuropsicólogo, salud mental y Rehabilitación Visual. La atención personal para las ABVD se incorporó porque es una variable necesaria y fundamental que apoya a la persona en la realización de las ABVD que no puede realizar, contribuyendo a que la persona pueda acudir a RHB integral. (ejemplo: ayuda para vestirla, darle de comer, entre otros).

Se destaca que solo la mitad de los pacientes que necesitan rehabilitación integral la reciben. Los recursos destinados a la rehabilitación del paciente con discapacidad moderada-grave son extremadamente limitados en número y accesibilidad ${ }^{194}$. La ausencia de centros públicos específicos en todas las localidades de residencia de los pacientes imposibilita el acceso de estos al tratamiento rehabilitador integral. En toda Europa hay especial falta de terapia ocupacional, logopedia y fisioterapia ${ }^{53}$, y no existe trabajo social especializado. Esta desigualdad implica que tengan que utilizar sus propios recursos personales para acceder a dicha rehabilitación.

\section{FACTORES ASOCIADOS AL ACCESO A REHABILITACIÓN INTEGRAL POST-ICTUS}

Los factores asociados claramente a la posibilidad de acceso a la rehabilitación integral en el primer estudio. En primer lugar, la edad que, con una correlación perfecta, evidencia que a medida que aumenta la edad disminuye en la misma proporción la posibilidad de acceder a dicha rehabilitación. La frecuencia de padecer ictus aumenta progresivamente a partir de los 55 años $^{78}$. Además, hay estudios que sugieren que una edad elevada impide el tratamiento rehabilitador pautado, al beneficiarse del mismo en mayor medida los jóvenes ${ }^{195}$, por lo que la edad constituye un factor determinante de la rehabilitación integral. El acceso a un tratamiento rehabilitador de acceso gratuito está limitado por la edad en ciertos centros. En los centros concertados, como el Centro Estatal de Atención Daño Cerebral (Ceadac) ${ }^{93}$, está limitado hasta los 55 años, y en Guttmann hasta los 60 años. 
El lugar de residencia (urbana o rural), es el segundo factor claramente asociado a la posibilidad de recibir una rehabilitación integral, en tanto que la accesibilidad a servicios sanitarios y sociosanitarios depende en buena parte de la proximidad y facilidad de acceso a los mismos (distancia/transporte).

Estos factores también son confirmados en el análisis univariante del segundo estudio. El lugar de residencia se confirmó en la regresión logística no así la edad.

En el segundo estudio, aunque en la regresión logística la edad no resulto significativa, se confirmó que el porcentaje de pacientes que menos accedió a la RHBI se encontraba en la franja mayor de 81 años. Esto contrasta con el estudio realizado ${ }^{196}$ por diversos autores que determina la edad es un factor asociado a una peor recuperación funcional, especialmente en edades avanzadas. Con frecuencia, la edad mayor de 85 años parece suponer un punto de inflexión en la capacidad de recuperación de un ictus, aunque diversos estudios enfatizan el hecho de que una menor capacidad de respuesta al tratamiento rehabilitador no es sinónimo de su beneficio, no debiendo ser la edad avanzada un factor que límite el acceso a dicho tratamiento.

Además, en el segundo estudio, en el cual se ampliaron las variables de acceso, quedo confirmado en la regresión logística, el Rankin y la modalidad de tratamiento rehabilitador privado como factores significativos en el acceso a la RHB integral. En un contexto donde los tratamientos rehabilitadores integrales públicos no están garantizados, el estudio muestra el uso de tratamientos privados para la recuperación (con la capacidad de pago que eso conlleva por parte del paciente o su entorno). El Rankin mostro relación con el acceso a la RHB integral, como principal herramienta para la valoración funcional tras un evento cerebrovascular ${ }^{197}$, es un criterio de acceso para tratamientos rehabilitadores

Ahora bien, en relación con el sexo que ha sido un factor que no presenta en ningún análisis una asociación significativa en el acceso a la RHBI $(p=0,183)$ se corrobora en el segundo estudio. El hecho de que el sexo no sea un factor significativo coincide con otros estudios que señalan que la capacidad funcional post-ictus al alta es similar en hombres y mujeres ${ }^{198}$.

Respecto a los ingresos económicos, parecía obvio que, en un contexto de falta de acceso gratuito y universal a la rehabilitación integral, y considerando que bastantes de los servicios necesarios debían proporcionarse a nivel privado-mercantil (mediante pago), los ingresos mensuales podrían condicionar el acceso a los mismos. Sin embargo, esto no ha sido así en la muestra estudiada, debido a que la diferencia de ingresos medios entre 
quienes reciben rehabilitación y quienes no la reciben, es muy pequeña (diferencia de $45,34 €$ en primer estudio y $57,81 €$ en segundo estudio).

Se constata, por tanto, que estos condicionantes sociales y ambientales (edad, lugar de residencia, modalidad de tratamiento privado) son marcadores de desigualdad en salud, tanto por el acceso diferencial al tratamiento rehabilitador integral post-ictus, como por los efectos que dicho tratamiento genera a largo plazo, en términos de autonomía funcional y esperanza de vida con calidad.

La equidad esté garantizada solo facilitando el acceso al sistema sanitario: hay que tener en cuenta las condiciones económicas, sociales y del entorno en que viven y se relacionan las personas. Lograr la equidad en salud requiere de un enfoque integral. El artículo 3 de la Ley General de Sanidad ${ }^{199}$ establece que el acceso a las prestaciones sanitarias se realizará en condiciones de igualdad efectiva y que las políticas de salud estarán orientadas a la superación de los desequilibrios sociales y territoriales. Algo que, en la actualidad y en el ámbito objeto de estudio, no se produce.

Por tanto, estos factores de desigualdad territorial y socioeconómica condicionan fuertemente el acceso a la rehabilitación integral y, con ello, la probabilidad de alcanzar una autonomía funcional a largo plazo.

\section{EFECTOS DE LA REHABILITACIÓN INTEGRAL POST-ICTUS A LARGO PLAZO}

Se ha demostrado que la RHB integral continuada desde el alta durante el primer año tras el ictus reduce el riesgo de deterioro funcional y mejora la independencia en las actividades de la vida diaria ${ }^{200}$, pero los programas rutinarios de RHB en pacientes de más de un año de evolución no han demostrado una eficacia significativa ${ }^{201}$. Nuestra investigación aporta evidencias acerca de los efectos de un tratamiento rehabilitador integral a largo plazo. Se evidencia que la RHB integral tras el ictus tiene un impacto pronóstico que se mantiene a largo plazo, en forma de una mejor situación funcional de los pacientes que acceden a la misma.

Se confirma la mejora en la funcionalidad del paciente, esto lo demostró el Barthel, la evolución funcional del Barthel aplicado en el año 2015 al que se aplicó en el año 2020 muestra claramente una evolución funcional de los pacientes, ya expuesto anteriormente. Esta mejora permite a los pacientes (entre otros efectos para su estado de salud), la permanencia en su entorno habitual, el desempeño de una actividad laboral y la capacidad para realizar actividades de la vida diaria por sí mismos, disminuyendo la necesidad de 
apoyos formales e informales por dependencia funcional y la institucionalización en centros residenciales.

En el modelo de regresión logística multivariante, resultaron como predictores de mejor pronóstico funcional las siguientes: acceso a rehabilitación integral, Rankin al alta y la menor distancia en kilómetros al centro de rehabilitación.

Por tanto, los condicionantes sociales y ambientales son marcadores de desigualdad en salud, tanto por el acceso diferencial al tratamiento rehabilitador integral post-ictus, como por los efectos que dicho tratamiento genera a largo plazo, en términos de autonomía funcional y esperanza de vida con calidad.

\section{LABOR DEL TRABAJO SOCIAL NEUROLÓGICO EN EL ACCESO Y CUMPLIMIENTO DEL TRATAMIENTO REHABILITADOR INTEGRAL POST-ÍCTUS}

El trabajo social neurológico tiene un papel clave en el acceso y durante el tratamiento RHB integral de pacientes que sufren ictus, por los siguientes motivos:

\subsection{Visión integral}

El programa rehabilitador no debe separarse, ni reiniciarse tratamientos inadecuados ${ }^{57} \mathrm{y} / \mathrm{o}$ disgregados por área funcional afectada, debiendo responder y englobar todas las áreas afectadas de la persona, de una manera integral.

El trabajador/a social es:

- Profesional integrador de una visión integral.

- Potenciador de encuentros multidisciplinares que propicien decisiones conjuntas del equipo multidisciplinar y den respuesta a la atención integral que precisa la persona. Contribuye a incorporar todos los aspectos relacionados con la situación del paciente y su familia e incorporarse al Plan Rehabilitador Integral Post-alta hospitalaria.

\subsection{Barreas sociales de acceso y mantenimiento del tratamiento rehabilitador integral post-ictus}

El trabajo social neurológico conoce e interviene en la situación personal, familiar, económica, tratando de corregir las dificultades que implican desigualdad en el acceso a servicios que precisan. Interviene socialmente movilizando a la persona, familia y recursos que permita superar las barreras que impiden una equidad en la salud. 


\subsection{Manejo de los recursos al alta hospitalaria}

La ausencia de centros rehabilitadores integrales públicos en los Sistemas Públicos de Salud, y las dificultades de acceso a los que existen (distancia geográfica, criterio inclusión de edad, necesidad de desplazamientos geográficos), hace necesaria la intervención del trabajo social neurológico. El trabajador social neurológico es responsable en la gestión y tramitación del tratamiento rehabilitador al alta del paciente. Su conocimiento de los tratamientos y procedimiento de acceso contribuye a posibilitar el acceso al mismo.

\subsection{Motivación-empoderamiento del paciente-familiar}

El trabajador social neurológico contribuye durante el acceso y tratamiento rehabilitador a:

1. Involucración activa del paciente y cuidadores en su proceso de rehabilitación desde el inicio del ingreso hospitalario en Servicio Médico de Neurología.

2. Integración de pacientes, familiares y cuidadores en el equipo de rehabilitación e imprescindibles en las decisiones que se deriven del proceso de rehabilitación.

\subsection{Seguimiento social continuo}

En toda Europa el apoyo y seguimiento a largo plazo continuos son insuficientes ${ }^{53}$. Esta falta de acceso al tratamiento rehabilitador integral y un seguimiento continuo a largo plazo provoca falta de calidad de vida y mayor gasto económico a la persona y a los Sistemas Públicos de Protección ${ }^{93}$, además de evidenciar la inequidad en salud:

- El trabajo social neurológico garantiza una atención psicosocial a pacientes y familiares durante el tratamiento rehabilitador

- Seguimiento de riesgos sociales que se produzcan durante el tratamiento rehabilitador y que repercuten en el buen desarrollo del mismo.

- Reevalúa la intervención social de forma periódica y la adapta según los condicionantes sociofamiliares y económicas que influyen en el tratamiento rehabilitador.

Por todo ello, la falta de garantía pública de acceso a la rehabilitación integral con carácter gratuito y universal en nuestro país en la mayoría de las comunidades autónomas, los determinantes sociofamiliares y económicos que influyen en el acceso a tratamientos privados y la ausencia de seguimiento en los Sistemas Públicos de Salud ${ }^{93}$. Estas 
situaciones hacen que la "gestión de caso social" desarrollada por el trabajador sanitario de neurología resulte determinante para implementar dicha rehabilitación integral, al movilizar diversos y múltiples recursos sociales, naturales, institucionales y privados, que permiten la rehabilitación integral del paciente susceptible de recibirla en territorios sin los recursos públicos adecuados. Promueve el seguimiento del tratamiento rehabilitador, contribuyendo a la recuperación del paciente y a garantizar una vida lo más autónoma posible

A tenor de los resultados de esta investigación que muestran que la recuperación integral de la persona está condicionada no solo por variables clínicas, sino por variables sociales, que resultan imprescindibles para su recuperación integral. El trabajo social presenta una serie de propuestas que considera contribuyen a superar estas dificultades en la recuperación integral de la persona que sufre ictus:

1. Entrenamiento específico a los cuidadores durante la rehabilitación de los pacientes que han sufrido ictus, esto reduce coste y carga cuidador.

2. El establecimiento de sesiones conjuntas periódicas del equipo neurorehabilitador que promuevan establecer un plan terapéutico de intervención, fijar los objetivos y realizar el seguimiento de la evolución del paciente.

3. Crear y mantener programas de apoyo social para pacientes con enfermedades neurológicas, programas de rehabilitación que incluyan los siguientes aspectos:

- Educación de la población en general que acepten al paciente neurológico como miembro de la sociedad con derechos normales y que no sea objeto de marginación.

- Ofertar programas de apoyo a familias que cuidan pacientes neurológicos, incluyendo programas de respiro familiar.

4. Asistencia y participación del paciente/familia a las reuniones multidisciplinares de análisis y evaluación del plan post-hospitalización al alta hospitalaria.

Y fundamentalmente, un perfil específico del trabajador/a social en el campo neurológico, con una amplia experiencia y una formación específica acreditada en este campo.

\section{LIMITACIONES Y PROSPECTIVA}

Las limitaciones de este estudio han sido considerables, ya que en Castilla y León todavía no está implantada en su totalidad la historia clínica electrónica del paciente, lo que ha supuesto tener que manejar varias herramientas informáticas de recopilación de datos. 
Las escalas aplicadas por la trabajadora social de neurología del HCUV no estaban incluidas en la herramienta informática, lo que ha implicado tener que incorporarlas manualmente. Esto prolongó notablemente el proceso de toma de datos. Otra limitación importante fue la dificultad de acceso a datos económicos precisos de los pacientes que estaban ingresados en el Hospital, la búsqueda activa de familiares (algunos fuera de la localidad, algunos sin relación con el paciente) para conseguir datos, y la gran dificultad en el caso de pacientes de los que al ingreso se temía por su vida. Hubo que realizar un trabajo de investigación y recopilación de datos en los casos en los que el paciente no podía expresarse. En otros casos, se contó con la ayuda de un logopeda y con un tablero de comprensión para poder realizar una entrevista

Este estudio tiene ciertas limitaciones, derivadas de un diseño no randomizado. Se refiere a pacientes ingresados de los que una proporción muy reducida no fueron incluidos: se trata de algunos pacientes menos graves, cuya estancia hospitalaria fue inferior a tres días. Además de los que fallecieron durante el período de hospitalización y los que han fallecido a lo largo de estos 5 años. Por ello, el tamaño muestral es limitado. Otra limitación es que el pronóstico funcional está basado solo en el Barthel, por ser la escala utilizada al alta hospitalaria en 2015. Para asegurar la validez de los datos comparativos se empleó la misma escala en 2020. Habría sido idóneo tener el Rankin a los 5 años de los pacientes, no ha sido posible, los pacientes no son seguidos en la consulta de Neurología durante más de $1-2$ años como máximo. Además, según una revisión de la literatura $^{202}$ acerca de la evaluación de las propiedades psicométricas de la escala de Rankin, se critica el uso de esta escala por ser subjetiva y porque la información relativa a su capacidad de respuesta al cambio es limitada. Hemos considerado que el Índice Barthel es más completo porque refleja a través de la comprobación el desenvolvimiento de la persona en las actividades básicas de la vida diaria. En el futuro podrían utilizarse otras escalas de calidad de vida o medidas pronosticas aportadas por los pacientes.

La ausencia de la figura del trabajo social neurológico en nuestro país ha impedido encontrar estudios de investigación relacionados con la tesis desde el trabajo social. Esto ha incrementado el nivel de complejidad de este estudio, no pudiendo comparar los resultados de nuestra investigación con los de otras investigaciones en la misma línea. Esto ha supuesto un reto para el investigador por la ausencia de referencias científicas en este tema desde el trabajo social en el campo neurológico.

El tamaño de la población objeto de estudio constituye una importante limitación, dando lugar a resultados que pueden aparecer como no significativos, lo que dificulta la 
comparación con otros estudios y la generalización de los resultados obtenidos. Por ello se requiere mantener esta línea de investigación solventando estos aspectos.

En el futuro inmediato se continuarán investigando otros efectos sociosanitarios y laborales del tratamiento rehabilitador integral, tanto en los pacientes de ictus como en la comunidad. 


\section{Capítulo 6.}

\section{CONCLUSIONES}


Teniendo en cuenta las hipótesis de partida y los objetivos de investigación derivados de las mismas, una vez analizados los resultados obtenidos, se formulan las siguientes conclusiones.

1. Casi la mitad de los pacientes con ictus considerados candidatos a recibir RHB integral en nuestro medio no logra acceder a la misma. El hecho que solo la mitad de la muestra hayan accedido a la misma, supone un serio problema sociosanitario que impide la posibilidad de recuperación de los pacientes candidatos a rehabilitación integral.

2. Los factores asociados de forma independiente a un peor acceso a RHB integral son la residencia en medio rural, una edad más avanzada, un mayor grado de discapacidad previa al ictus, y la necesidad de complementar el tratamiento rehabilitador público con recursos privados. La influencia de estos factores sociales en el acceso a la RHB integral evidencia la función clave del trabajo social neurológico para superar la desigualdad derivada de dichos factores en el acceso al tratamiento RHB integral de la persona que sufre ictus.

3. Se demuestran los efectos positivos a largo plazo (5 años) del tratamiento rehabilitador integral en la autonomía funcional de los pacientes, aportando evidencia científica del beneficio a largo plazo de la RHB integral para el aumento y mejora de la autonomía funcional de la persona, considerada tanto en su capacidad de desempeño de las Actividades Básicas de la Vida Diaria (ABVD) como de su capacidad relacional con el entorno de convivencia. 


\section{BIBLIOGRAFIA}

1. Organización Mundial de la Salud. ¿Qué son los trastornos neurológicos? [Internet]. 2016 [citado el 4 de febrero 2021]. Recuperado a partir de https://www.who.int/features/qa/55/es

2. Corona-Vázquez T, Campillo-Serrano C, López M, Mateos-G JH, Soto-Hernández, JL. Las enfermedades neurológicas I. Su dimensión y repercusión social II. Depresión y demencia III. Trauma y tumores IV. Infecciones del Sistema Nervioso Central. Gac Méd Méx [Internet]. 2002 [citado el 4 de febrero 2021];138(2):533-546. Recuperado a partir de: http://www.anmm.org.mx/bgmm/1864_2007/2002-138-6-533-546.pdf

3. Organización Mundial de la Salud. Los trastornos neurológicos afectan a millones de personas en todo el mundo: informe de la OMS [Internet]. 2007 [citado 20 de febrero de 2021]. Recuperado a partir de: $h \mathrm{ttps}: / / \mathrm{www} . w h o . i n t / m e d i a c e n t r e / n e w s /$ releases/ 2007/pr04/es/

4. Suárez-Escudero JC. Discapacidad y neurociencias: la magnitud del déficit neurológico y neuropsiquiátrico. Acta Neurol Colomb [Internet]. 2014 [citado 2 de febrero 2021];30(4):290-299. Recuperado a partir de https://www.researchgate.net/publication/313029412 Discapacidad y_neurociencias la _magnitud_del_deficit_neurologico_y_neuro_psiquiatrico

5. Mar J, Alvárez-Sabin J, Oliva J, Becerra V, Casado MA, Yébenes M, et. al. Los costes del ictus en España según su etiología. El protocolo del estudio CONOCES. Neurologia [Internet]. 2013 [citado el 4 de febrero 2021];28(6):332-339. Recuperado a partir de https://www.elsevier.es/es-revista-neurologia-295-articulo-los-costes-del-ictusespana-S0213485312002447 
6. González-Aquines A, Martinez-Roque A, Baltazar A, Chávez-Luévanos F, GuerreroCampos F, Góngora F. Síndrome de apnea obstructiva del sueño y su relación con ictus isquémico. Rev.Neurol.2019;69:255-260. doi.org./10.33588/rn.6906.2019061

7. Plaza I. ¿Qué es un ictus? En Castellvi E. et al. Guía de información al paciente con ictus .Valencia: Generalitat. Conselleria de Sanidad; 2007. p.13-16.

8. Arenillas Lara JF, Molina Cateriano CA (dir), Álvarez Sabin J (dir). Arterosclerosis Intracraneal de Gran Vaso: Progresión y Recurrencia clínica [tesis doctoral en Internet]. [Barcelona]: Universidad Autónoma de Barcelona, 2003 [citado el 4 de febrero 2021]. Recuperado de https://ddd.uab.cat/pub/tesis/2003/tdx-1020104-120347/jfal1de1.pdf

9. Feigin VL, Forouzanfar MH, Krishnamurthi R, Mensah GA, Connor M, Bennett DA, et al. Global and regional burden of stroke during 1990-2010: Findings from the Global Burden of Disease Study 2010. Lancet. 2014;383(9913):245-55. doi: 10.1016/s01406736(13)61953-4.

10. Lopez-Espuela F, González-Gil T, Jiménez, MA, Bravo-Fernández S, AmarillaDonoso, J. Impacto en la calidad de vida en cuidadores de supervivientes de un ictus. Enfermeria Clínica [Internet] 2015;25(2):49-56.

https://doi.org/10.1016/j.enfcli.2014.12.008

11. Garcia Culebras A, Lizasoain Hernández I (dir.), Moro Sánchez MA (dir.), Moraga Yébenes A (dir). Estudio del papel de TLR4 en la transformación hemorrágica e infiltración aguda tras ictus experimental [tesis en internet]. [Madrid]: Universidad Complutense de Madrid;2017 (citado 4 de febrero del 2021). Recuperado de https://dialnet.unirioja.es/servlet/tesis? codigo $=129392$

12. Noé-Sebastián E, Balasch-Bernat M, Colomer-Font C, Moliner-Muñoz B, Rodríguez C, Ugartp P, et al. Ictus y discapacidad: estudio longitudinal en pacientes con discapacidad moderada-grave tras un ictus incluido en un programa de rehabilitación multidisciplinar. Rev Neurol [Internet]. 2017 [citado 4 de febrero 2021];64(9):385-392. Recuperado a partir de http://www.nrhb.webs.upv.es/wpcontent/uploads/2017/06/Ictus_Neurologia.pdf

13. Olmedo V, Arranz M. Trabajo Social Sanitario: Intervención con pacientes con ictus desde el Servicio Médico de Neurología del Hospital Clínico Universitario de Valladolid. Trab Soc Salud. 2017;88:29-47.

14. Arias A. Rehabilitación del ACV: evaluación, pronóstico y tratamiento. Galicia Clin [Internet]. 2009 [citado 4 de febrero 2021];70(3):25-40. Recuperado a partir de https://galiciaclinica.info/ PDF/5/81.pdf

15. Flynn RWV, MacWalter RSM, Doney ASF. The cost of cerebral ischaemia Neuropharmacology. 2008;55(3):250-256. https://doi.org/10.1016/j.neuropharm.2008.05.031

16. Sociedad Española de Neurología. Impacto de las enfermedades neurológicas en la mortalidad española: LXX Reunión Anual de la Sen [Internet]. 2018 [citado el 4 de febrero 2021]. Recuperado a partir de http://www.sen.es/saladeprensa/pdf/Link257Informe.pdf

17. Campbell BC, De Silva DA, Malcolm RM, Coutts B, Schwamm LH, Davis SM et al. Ischaemic stroke. Nature Reviews Disease Primers [Internet]. 2019 [citado el 4 de febrero de 2021];5(70). https://doi.org/10.1038/s41572-019-0118-8 
18. Herrera J, Prieto C y Martin R. Las intervenciones con familias desde atención primaria de salud por el personal sanitario. Trab Soc Salud;57:281.

19. Ituarte A. Procedimiento y proceso en trabajo Social Clínico. Madrid, Siglo XXI y Consejo General de Diplomados en Trabajo Social y Asistentes Sociales; 1992

20. Dobkin BH, Carmichael S. Principles of recovery after stroke. In: Barnes M, Dobkin B, Bogousslavsky J, eds. Recovery after Stroke. United Kingdom: Cambridge University Press; 2005. p.47- 53.

21. Cauraugh J, Light K, Kim S, Thigpen M, Behrman A. Chronic motor dysfunction after stroke: recovering wrist and finger extension by electromyography-triggered neuromuscular stimulation. Stroke. 2000;31(6):1360-4.

https://doi.org/10.1161/01.STR.31.6.1360

22. Morris DM, Taub E, Mark VW. Constraint-induced movement therapy: characterizing the intervention protocol. Eura Medicophys [Internet]. 2006 [citado 5 de febrero 2021];42(3):257-68. Recuperado a partir de https://www.uab.edu/citherapy/images/CIT training/constraintinduced movement therapy characterizing the intervention protocol.pdf

23. Bonita R, Solomon N, Broad JB. Prevalence of stroke and stroke-related disability. Estimates from the Auckland stroke studies. Stroke.1997;28(10):1898-902. https://doi.org/10.1161/01.STR.28.10.1898

24. Olmedo V, Diez S. El Acompañamiento social a pacientes que sufren ictus durante su ingreso hospitalario. Ocronos. Revista Médica y de Enfermeria [Internet]. 2020 [citado el 15 de marzo de 2021] Recuperado a partir de https://revistamedica.com/acompanamiento-social-ictus-ingreso-hospitalario/

25. Moltó JM, Lozano A, Castellví E, y Villanueva V. Las Ayudas. Recursos Sociales tras un Ictus. En Castellvi et al. Guía de información al paciente con ictus. Valencia: Generalitat Valenciana. 2007. p. 103-107.

26. Masjuan J, Álvarez-Sabín J, Arenillas J, Calleja S, Castillo J, Dávalos A, et al. Plan de asistencia sanitaria al ICTUS II. 2010 Stroke health care plan (ICTUS II. 2010). Neurología. 2011;26(7):383-396. https://doi.org/10.1016/..nrl.2010.05.008

27 Álvarez-Sabín J, Quintana M, Masjuan J, Oliva-Moreno J, Mar J, González-Rojas N, et al. Economic impact of patients admitted to stroke units in Spain. Eur J Health Econ. 2017;18:449-458. doi 10.1007/s10198-016-0799-9

28. Edwardson MA. Ischemic stroke prognosis in adults. [Internet]. 2016 [citado 3 de febrero 2021];1-29. Recuperado a partir de:

https://www.uptodate.com/contents/overview-of-ischemic-stroke-prognosis-in-adults

29. Atehortua M, Suárez-Escudero JC. Discapacidad visual y ceguera: definición, etiología neurológica y mecanismos de rehabilitación. Rev. Sociedad Colombiana de Oftalmología 2012, Abril-Junio;45(2):138-147.

30. Dickey L, Kagan A, Lindsay MP, Fang J, Rowland A, Black S. Incidence and Profile of Inpatient Stroke-Induced Aphasia in Ontario, Canada. Arch Phys Med Rehabil. 2010;91(2):196-202. https://doi.org/10.1016/j.apmr.2009.09.020 
31. Sánchez Tarifa, MP, Álvarez Badillo A (dir), Palomino Aguado B (dir.) y Jiménez Cosmes L (dir). Contribución de la rehabilitación en pacientes de la unidad de ictus en términos de discapacidad y calidad de vida [Tesis en internet]. [Madrid]: Complutense de Madrid;2018 [citado el 4 de febrero 2021]. Recuperado a partir de:

https://dialnet.unirioja.es/servlet/tesis? codigo $=231351$

32. Youman P, Wilson K, Harraf F, Kalra L. The economic burden of stroke in the United Kingdom. Pharmacoeconomics. 2003;21:43-50. doi: 10.2165/00019053$\underline{\text { 200321001-00005 }}$

33. Mar J, Arrospide A, Begiristain JM, Larrañaga I, Elosegui E, Oliva-Moreno J. The impact of acquired brain damage in terms of epidemiology, economics and loss in quality of life. BMC Neurol. 2011;11:46. https://doi.org/10.1186/1471-2377-11-46

34. Schlesinger LE. Staff authority and patient participation in rehabilitation. In J. Stubbins (Ed.). Social and psychological aspects of disability. A handbook for practitioners Baltimore: University Park Press; 1997. p. 167-172.

35. Lehnerer S, Hotter B, Padberg I, et al. Social work support and unmet social needs in life after stroke: a cross-sectional exploratory study. BMC Neurol.2019;19(1):220. doi: 10.1186/s12883-019-1451-a.

36. Seelman K. Trends in Rehabilitation and disability: transition from a medical model to an integrative model [Internet]. University of Pittsburgh Pittsburgh, PA. U.S.A. 2003 [citado 10 de febrero 2021]. 31p. Recuperado a partir de: https://www.normanet.ne.jp/ rehab/2003/seelman.pdf

37. Céspedes G. La nueva cultura de la discapacidad y los modelos de rehabilitación. Aquichan [Internet]. 2005 [citado el 3 de marzo 2021];5(1). Recuperado a partir de: http://www.scielo.org.co/scielo.php?script $=$ sci_arttext\&pid $=$ S1657$\underline{59972005000100011}$

38. Valero M. Modelo de Rehabilitación Integral. Revista Colombiana de Rehabilitación. 2006;5(1):34-42. https://doi.org/10.30788/RevColReh.v5.n1.2018.282

39. Phillips S. A rehabilitation process model. Journal of Rehabilitation. 1980;46(1):4245 .

40. Gómez L, y Valencia M. Resumen del estudio nacional de necesidades, oferta y demanda de servicios de rehabilitación en Colombia, 2003 [Internet]. Bogotá:

Fundación Saldarriaga Concha; 2003 [citado 20 febrero 2021]. Recuperado a partir de https://www.saldarriagaconcha.org/wpcontent/uploads/2019/01/02 estudio nal nec of y demand.pdf

41. Angarita Rodríguez C, Alvis Gómez K (dir). Rehabilitación integral: Un análisis de contenido del marco normativo de apoyo a la discapacidad [tesis doctoral en internet]. [Colombia]: Universidad Nacional de Colombia; 2014 [citado 20 febrero 2021]. Recuperado a partir de: https://repositorio.unal.edu.co/bitstream/handle/unal/21820/dianacristinaangarita.2014.p df? sequence $=1 \&$ isAllowed $=y$

42. Albrecht G. The disability business: Rehabilitation in America. Hunter JL, editor. Newbury Park: Sage Publications;1992. 336P. 
43. Muyor J. Entre Bioética, Biopolitica y ética profesional: Sobre el proceso de discapacidad como constructo y su relación con el trabajo social. Trab Soc Salud. 2010;66:283-285.

44. Boada J. Rehabilitación integral: Un concepto de ayer es una necesidad hoy. Revista Fundamentos. 2004:36-39.

45. Millán Muñoz R. Modelos de rehabilitación ideal. [Monografía en Internet]. Cali (Colombia): Fundación Ideal Julio H. Calonja; 2012 [consultado 4 de febrero 2021]. Recuperado de: https://www.fundacionideal.org.co/publicaciones/modelo-derehabilitacion-integral-ideal

46. Stroke Unit Trialists'Collaboration Organised inpatient (stroke unit) care for stroke. Cochrane Database Syst Rev. 2013(9):CD000197.

doi: 10.1002/14651858.CD000197.pub3

47. Sociedad Española de Neurorrehabilitación. Noé Sebastián E, Gómez Blanco A, Bernabeu Guitart M y Quemada Ubís I. Guía neurorrehabilitación [Internet]. 2020 [citado el 4 de febrero 2021]. 76p. Recuperado a partir de https://www.neuroreha.com/pdf/Guia_SENR_2_Atencion_del_DCS.pdf

48. Olmedo V, González R. Repensando la intervención psicosocial con pacientes ictus. Revista Investigación Acciones Sociales [Internet]. 2018 [citado el 4 de marzo de 2021];39:89-110. Recuperado a partir de:

https://sociales.unizar.es/sites/sociales.unizar.es/files/users/sociales/AIS/39_AIS/ais39 04.pdf

49. National Association of Social Workers (NASW). Standars for the Classification of Social Work Practice, policy statement 4. Silver Spring, MD: Author; 1981. 34 p.

50. National Association of Social Workers (NASW). Standars for Social Work Services in Long- Term Care Facilities. Professional Standards [Internet]. MD: Nasw; 2003 [citado el 21 de marzo de 2021]; 24 p.

Recuperado a partir de:

https://www.socialworkers.org/LinkClick.aspx?fileticket $=\% 20 \mathrm{cwW} 7 \mathrm{zBfYxg} \% 3 \mathrm{~d} \& \mathrm{port}$ $\underline{\text { alid }=0}$

51. Lynch EA, Luker JA, Cadilhac DA, Fryer CE, Hillier SL. A qualitative study using the Theoretical Domains Framework to investigate why patients were or were not assessed for rehabilitation after stroke. Clin Rehabil. 2017;31(7):966-77.

https://doi.org/10.1177/0269215516658938

52. Pla director de la malaltia vascular cerebral. 4rt. Audit Clínic de l'ictus [Internet]. Catalunya 2013.2014 [citado 3 de marzo 2021]. 43p. Recuperado a partir de:

https://canalsalut.gencat.cat/web/.content/A-Z/I/ictus/prof/documents/arxius/Informe ACI4_CORR.pdf

53. Murie-Fernández $\mathrm{M}$, Laxe $\mathrm{S}$. Comparación de la neurorrehabilitación del ictus en España y en Europa. Neurologia 2020;35(5):354-5. doi:10.1016/j.nrl.2018.02.007

54. Herbert D, Lindsay MP, MCIntyre A, Kirton A, Rumney PG, Bagg S et al. Canadian stroke best practice recommendations: Stroke rehabilitation practice guidelines, update 2015. Int J Stroke (Internet) 2016;11(4):459-84. doi: $\underline{10.1177 / 1747493016643553 .}$ 
55. NICE. Stroke Rehabilitation: Long term rehabilitation after stroke. National Clinical Guideline Centre [Internet]. 2013 [citado el 20 de febrero 2021]: 1-42. Recuperado a partir de: https://www.nice.org.uk/guidance/cg162/documents/stroke-rehabilitation-2ndguideline-consultation-nice-guideline2

56. Arcos Garcia J, Aguilera Manrique G (tutor). Unidades de rehabilitación de Ictus. Situación en España. Aproximación al Rol Enfermero [trabajo fin de grado en internet]. [Almería]: Universidad de Almeria;2017 [citado 20 de febrero 2021]. Recuperado a partir de http://repositorio.ual.es/bitstream/handle/10835/6515/13972 Unidades \%20de $\% 20$ Rehabilitación $\% 20 \mathrm{de} \% 20$ Ictus..pdf? sequence $=1 \&$ isAllowed $=\mathrm{y}$

57. Duarte E, Alonso B, Fernández MJ, Fernández JM, Flórez M, García-Montes I, et al. Rehabilitación del ictus: modelo asistencial. Recomendaciones de la Sociedad Española de Rehabilitación y Medicina Física, 2009. Rehabilitación. 2010;44:60-68. doi: 10.1016/j.rh.2009.10.001

58. Palomino Aguado B, Rodríguez Rodríguez LP (dir), Jiménez Cosme L (dir). Contribución de la adaptación y validación de la escala SIS-16 (Stroke Impact Scale) en el manejo de la rehabilitación de pacientes con ictus [tesis en Internet]. [Madrid]: Universidad Complutense de Madrid; 2010 [citado 1 de marzo 2021]. Recuperado a partir de: https://eprints.ucm.es/id/eprint/11558/

59. Woo J, Chan SY, Sum MW, Wong E, Chui YP. In patient stroke rehabilitation efficiency: influence of organization of service delivery and staff numbers. BMC Health Services Research. 2008;8:86. https://doi.org/10.1186/1472-6963-8-86

60. Mesa Y, Hernández TE, Parada Y. Factores determinantes de la calidad de vida en pacientes sobrevivientes a un ictus. Revista habanera de ciencias médicas [Internet]. 2017 [Citado el 20 de febrero 2021];16(5):735-750. Recuperado de:

http://www.revhabanera.sld.cu/index.php/rhab/article/view/2021

61. Vázquez Guimaraens M, Cudeiro Mazaira FJ (codir), Caamaño Ponte JL (codir). Factores relacionados a una mayor recuperación funcional tras sufrir un accidente cerebrovascular [tesis doctoral en Internet]. [Galicia]: Universidad de la Coruña; 2017 [citado a 20 febrero 2021]. Recuperado a partir de: https://dialnet.unirioja.es/servlet/tesis?codigo $=121181$

62. Ministerio de la Protección Social. "Manual de Procedimientos para la Rehabilitación y Reincorporación Ocupacional de los Trabajadores en el Sistema General de Riesgos Profesionales"[Internet]. Colombia: Ministerio de la Protección Social; 2010. p.33,49-53. Recuperado a partir de: https://www.libertycolombia.com.co/sites/default/files/201907/Manual\%20para\%20rehabilitacion\%202012.pdf

63. Instituto Salvadoreño de Rehabilitación Integral ISRI. "Centro de Rehabilitación Profesional: Servicios que prestan a la población" [Internet]. 2017. [citado 20 de febrero de 2021]. Recuperado a partir de: http://www.isri.gob.sv/wpcontent/uploads/2017/03/SERVICIOS-ISRI-CENTRO-DE-REHABILITACIONPROFESIONAL.pdf

64. Verdugo MA. El papel de la Psicología de la Rehabilitación en la integración de personas con discapacidad y en el logro de calidad de vida. Siglo Cero. 2015; 25(6):3340 . 
65. Muñoz JM, Hodan RM, Everts F, Garcia A, Aguilera S. Evaluación y tratamiento del traumatismo craneoencefálico: Estudio neuropsicológico de un caso. Revista de casos clínicos en Salud Mental [Internet]. 2018 [citado 4 de marzo de 2021];1:51-70. Recuperado a partir de http://www.casosclinicosensaludmental.es/files/EVALUACI-NY-TRATAMIENTO-DEL-TRAUMATISMO-CRANEOENCEF-LICO--ESTUDIONEUROPSICOL-GICO-DE-UN-CASO-.pdf

66. Aguilar-Idáñez $\mathrm{M}^{\mathrm{a}}$. Las experiencias de éxito de promoción de la integración laboral de inmigrantes y colectivos hasta ahora excluidos total o parcialmente, mujeres, mayores y discapacitados. Revista del Instituto de Estudios Económicos. 2008;2:89-125.

67. Morris C. Measuring participation in childhood disability: How does the capability approach improve our understanding? Developmental Medicine \& Child Neurology. 2009;51:92-94. https://doi.org/10.1111/j.1469-8749.2008.03248.x

68. Van der Cruyssen K, Vereeck L, Saeys W, Remmen R. Prognostic factors for discharge destination after acute stroke: a comprehensive literature review. 177 Disabil Rehabil [Internet]. 2015 [citado el 21 de marzo de 2021];37(14):1214-27. Recuperado a partir de: http://www.ncbi.nlm.nih.gov/pubmed/25250810.

69. Begollin M. Factores económicos y sociales que influencian la salud y la enfermedad. Trab Soc Salud. 2008;60:189.

70. Putman K, De Wit L, Schupp W, Beyens H, Dejaeger E, de Weerdt W, et al. Inpatient stroke rehabilitation: a comparative study of admission criteria to stroke rehabilitation units in four European centres. Journal of Rehabilitation Medicine. 2007;39(1):21-6. doi:10.2340/16501977-0006

71. Hakkennes S, Hill KD, Brock K, Bernhardt J, Churilov L. Selection for inpatient rehabilitation after severe stroke: what factors influence rehabilitation assessor decisionmaking? Journal of Rehabilitation Medicine. 2013;45(1):24-31. doi:10.2340/16501977$\underline{1065}$

72. Hakkennes SJ, Brock K, Hill KD. Selection for inpatient rehabilitation after acute stroke: a systematic review of the literature. Arch Phys Med Rehabil.

2011;92(12):2057-70. doi: 10.1016/j.apmr.2011.07.189

73. New PW. The Assessment and Selection of Potential Rehabilitation Patients in Acute Hospitals: A Literature Review and Commentary. The Open Rehabilitation Journal. 2009;2:24-34. doi: 10.2174/1874943700902010024

74. Wade DT. Selection criteria for rehabilitation services. Clin Rehabil. 2003;17(2):115-8. https://doi.org/10.1191\%2F0269215503cr591ed

75. Abades M, Rayón E. El envejecimiento en España: ¿un reto o un problema social?,Gerokomos. 2012; 23(4) http://dx.doi.org/10.4321/S1134-928X2012000400002

76. Ministerio de Sanidad y Política Social. Estrategia en Ictus del Sistema Nacional de Salud [Internet]. Madrid: Ministerio de Sanidad y Política Social; 2008 [citado el 21 de marzo de 2021]. 163p. Recuperado a partir de:

https://www.mscbs.gob.es/organizacion/sns/planCalidadSNS/docs/EstrategiaIctusSNS.p $\underline{\mathrm{df}}$

77. Haheim LL, Holme I, Hjermann I, Leren P. Risk factors of stroke incidence and mortality: a 12 yearfollow-up of the Oslo study. Stroke. 2013; 24(10):1484-9.

78. Fernández-Gómez FJ, Hernández-Fernández F, Argandoña L, Galindo MF, Segura 
T, Jordan J. Farmacologia de la neuroprotección en el ictus isquémico agudo. Rev Neurol [Internet]. 2008 [citado el 21 de febrero 2021];47(5):253-260. Recuperado a partir de:

https://www.researchgate.net/publication/23246046_Pharmacology_of_neuroprotection in acute ischemic stroke

79. Petrea RE, Beiser AS, Seshadri S, Kelly-Hayes M, Kase CS, Wolf PA. Gender differences in stroke incidence and poststroke disability in the Framingham Heart Study.Stroke. 2009;40:1032-7. https://doi.org/10.1161/strokeaha.108.542894

80. Martinez Barandalla $C$, Ciga Lozano MA (codirector). Ictus: incidencia,, factores de riesgo y repercusión [Trabajo fin de grado en internet]. [Pamplona]: Universidad Pública de Navarra; 2014 [citado el 15 de marzo de 2021]. Recuperado a partir de: https://academica-e.unavarra.es/bitstream/handle/2454/16253/TFGCristinaMartinez Barandalla.pdf? sequence $=1$ \&isAllowed $=\mathrm{y}$

81. Rodríguez AJ, Pila Pérez R, Pila Peláez R, Vargas AR. Factores determinantes del pronóstico en el ictus isquémico. Revista Archivo Médico de Camagüey [Internet]. 2005 [citado el 10 de marzo 2021];9(2). Recuperado de http://scielo.sld.cu/scielo.php?pid=S1025$02552005000200003 \&$ script $=$ sci_arttext\&tlng=en

82. Santos-Lasaosa S, Mostacero E, Tejero C, López E, Ríos C, Morales F, et al. Pronóstico funcional a los tres meses en el paciente con ictus: factores determinantes. Rev Neurol. 1999;29:697-700. https://doi.org/10.33588/rn.2908.99308

83. Forrelli M, Alperovich A, Argentino C, Sachetti M, Toni D, Stte G, et al. For the Italian acute study group. Arch Neurol. 1995;52:250-5.

84. López-Martínez T, Bernardo-Cofiño J, Prieto-García E, Feito-Álvarez M, De DiosDel Valle R, Calleja-Puerta S. Diferencias de sexo en el abordaje integral del ictus en un área sanitaria rural de Asturias. Rev Neurol [internet]. 2016 [citado el 15 de marzo 2021];63:151-9. Recuperado a partir de:

https://www.researchgate.net/profile/Sergio_Calleja2 /publication/307470339 Sex differences in the integrated stroke management in a rural healthcare area in_Asturias/links/57de590108aeea195938d58a.pdf

85. Domínguez M, La división del trabajo doméstico en las parejas españolas. Un análisis del uso del tiempo. Revista internacional de sociología (RIS). 2012;70(1):153179. DOI:10.3989/ris.2009.08.26

86. Kapral MK, Fang J, Hill MD, Silver F, Richards J, Jaigobin C, et al. Sex differences in stroke care and outcomes: results from the Registry of the Canadian Stroke.Network. Stroke. 2005;36:809-814. doi: 10.1161/01.STR.0000157662.09551.e5

87. Dehlendorff C, Andersen KK, Olsen TS. Sex disparities in stroke: women have more severe strokes but better survival than men. Journal of the American Heart Association. 2015;4:e001967.

https://www.ahajournals.org/doi/10.1161/JAHA.115.001967

88. Haast RA, Gustafson DR, Kiliaan AJ. Sex differences in stroke. Journal of Cerebral Blood Flow \& Metabolism. 2012;32:2100-7.https://doi.org/10.1038\%2Fjcbfm.2012.141

89. Arboix A, Cartanya A, Lowak M, García-Eroles L, Parra O, Oliveres M, et al. Gender differences and woman-specific trends in acute stroke: Results from a hospital- 
based registry (1986-2009). Clin Neurol Neurosurg 2014;127:19-24.

doi: 10.1016/j.clineuro.2014.09.024

90. Koton S, Telman G, Kimiagar I, Tanne D. Gender differences in characteristics, management and outcome at discharge and three months after stroke in a national acute registry. International Journal of Cardiology. 2013;168:4081-4.

https://doi.org/10.1016/j.ijcard.2013.07.019

91. Alonso de Leciñana M, Egido JA, Casado I, Ribó M, Dávalos A, Masjuán J, et al. Guía para el tratamiento del infarto cerebral agudo. Neurologia 2014;29:102-22. doi: 10.1016/j.nrl.2011.09.012

92. Murie-Fernández M, Ortega-Cubero S, Carmona-Abellán M, Meyer M, Teasell R. Tiempo es cerebro, ¿solo en la fase aguda del ictus? Neurologia 2012;27:197-201. DOI: 10.1016/j.nrl.2011.06.007

93. Olmedo V, Idañez MJ, Arenillas JF. Análisis de factores asociados a la recuperación integral a pacientes de ictus al alta de Hospital de Agudos. Revista Española de Salud Publica [Internet]. 2019 [citado el 22 de marzo de 2021];93:1-13. Recuperado a partir de:

https://www.mscbs.gob.es/biblioPublic/publicaciones/recursos_propios/resp/revista_cdr om/VOL93/ORIGINALES/RS93C 201910103.pdf

94. Simo-Noguera CX, Hernández-Monleon A, Muñoz-Rodríguez D y González-San Juan ME. The Effect on Health of Marital and Cohabitation Status. El efecto del estado civil y de la convivencia en pareja en la salud. Revista Española de Investigaciones Sociológicas (REIS). 2015; 151(25):141-165. http://dx.doi.org/10.5477/cis/reis.151.141

95. Wade DT, Leigh-Smith J, Hewer RA. Depressed mood after stroke. A community study of its frequency. The British Journal of Psychiatry : the Journal of Mental Science,1987;151:200-5. doi: 10.1192/bjp.151.2.200

96. Flecher B. Work Stress, Disease and Life Expectancy. New York:Wiley;1991.255p.

97. ¡Rogers C.R., El poder de la persona, México: El Manual Moderno; 1980. 205 p.

98. Colman E, Van Praag L, Bracke P. Differences in mental health service consumption between the divorced and the married. Sixt Meeting of the European network for the Sociological and Demographic Study and Divorce, Abstracts. 2008.

99. Dupre ME, Meadows SO. «Disaggregating the Effects of Marital Trajectories on Health». Journal of Family Issues. 2007;28(5):623-652.

https://doi.org/10.1177\%2F0192513X06296296

100. Hughes ME, y Waite LJ. «Marital Biography and Health at Mid-life». J Health Soc Behav. 2009;50(3):344-358. doi:10.1177/002214650905000307

101. Hafen BH, Karren KJ. Mind/body Health: The Effects of Attitudes, Emotions, and Relationships. Boston: Allyn and Bacon. 1996. 660p.

102. IMSERSO (España). Cuidados a las personas mayores en los hogares españoles. El entorno familiar [Internet]. Madrid: Ministerio de Trabajo y Asuntos Sociales; 2005 [citado el 5 de marzo de 2021]. Recuperado partir de:

http://envejecimiento.csic.es/documentos/documentos/imserso-cuidados-01.

103. Costa MJ, Costa MP, Medeiros MO, Rodrigues $\mathrm{P}$, y Correia RJ. Anciano que cuida a anciano. Revista Gerokomos. 2007;18(3):127-134. 
104. Drefahl S. How does the age gap between partners affect their survival?. Demography. 2010;47:313-326. https://doi.org/10.1353/dem.0.0106

105. Ministerio de Trabajo y Asuntos Sociales. La soledad en las personas mayores: influencias personales, familiares y sociales. Análisis cualitativo. Madrid: Ministerio de Trabajo y Asuntos Sociales; 2001 [citado el 20 de marzo 2021]. 221p. Recuperado a partir de http://envejecimiento.csic.es/documentos/documentos/iglesias-soledad-01.pdf. PÁGINA 114-115.

106. Gaugler JE, Duval S, Anderson KA, Kane RL. Predicting nursing home admission in the US: a meta-analysis. BMG Geriatrics. 2007;7(13):1-14. doi: $10.1186 / 1471-2318-$ $7-13$

107. Ghäler M. «To Divorce is to Die a Bit...: A Longitudinal Study of Marital Distruption and Psychological Distress Among Swedish Women and Men». The Family Journal. 2006;14(4):372-382. doi:10.1177/1066480706290145

108. Solsona M. «Narrar la propia biografía después de un divorcio: notas de un estudio cualitativo de interés para la demografía». Estudios Geográficos. 2009;70(267): 633660. doi: 10.3989/estgeogr.0467

109. Wallerstein JS, Blakeslee S. What about the Kids? Raising your Children before, during, and after Divorce. New York: Hyperion; 2003. 380 p.

110. Amato PR. «The Consequences of Divorce for Adults and Children». Journal of Marriage and Family. 2000;62(4):1269-1287. https://doi.org/10.1111/j.17413737.2000.01269.x

111. Rutter M. «Psychosocial Resilience and Protective Mechanisms». American Journal of Orthopsychiatry. 1987;57(3):316-331. https://doi.org/10.1111/j.19390025.1987.tb03541.x

112. Kalmijn M. «Country Differences in the Effects of Divorce on Well-Being: The Role of Norms, Support, and Selectivity». Eur Sociol Rev. 2010;26(4): 475490.Recuperado a partir de: https://www.jstor.org/stable/40784574?seq=1

113. Clemente MA. Redes sociales de apoyo en relacion al proceso de envejecimiento humano revision bibliografica. Interdisciplinaria [Internet]. 2003 [Citado 22 de marzo 2021];20(1):31-60. Recuperado a partir de: https://es.slideshare.net/pedrito236/redessociales-de-apoyo-en-relacion-al

114. Hughes ME, Waite LJ. «Marital Biography and Health at Mid-life». Journal of Health and Social Behavior. 2009;50(3):344-358. https://doi.org/10.1177/002214650905000307

115. Williams K. «Has the Future of Marriage Arrived? A Contemporary Examination of Gender, Marriage, and Psychological Well-being». Journal of Health and Social Behavior. 2003;44(4):470-487. https://doi.org/10.2307/1519794

116. Lebrusan I. Más allá de la familia: Una reflexión teórica sobre la definición del habitante de la vivienda. Tendencias Sociales. Revista de Sociología, 3 (2019): 60-76. P.74. http://revistas.uned.es/index.php/Tendencias/article/view/23588/ 18947

117. Doblhammer G, Gumà J. Gender differences in the relationship between household position and health in twelve European countries: are they associated with the value climate?. En Doblhammer G, Gumà J, editors. A demographic perspective on 
gender, family and health in Europe. Rostock, Barcelona: Springer Open; 2018. p. 103131. https://doi.org/10.1007/978-3-319-72356-3

118. Waite L, Gallagher M. The case for marriage: why married people are happier, healthier and better off financially. New York: Doubleday. 2000.

119. Bastida-González R, Valdez-Medina JL, Valor-Segura I, González NI, RiveraAragón S. Satisfacción marital y estado civil como factores protectores de la depresión y ansiedad. Revista Argentina de Clinica Psicológica [Internet]. 2017 [citado 23 de marzo de 2021];24(1):95-102. Recuperado a partir de: https://www.redalyc.org/pdf/2819/281950399009.pdf

120. Godin G, Côté J, Naccache H, Lambert LD y Trottier S. Prediction of adherence to antiretroviral therapy: A one-year longitudinal study. AIDS Care. 2005;17: 493-504. https://doi.org/10.1080/09540120412331291715

121. Pratt RJ, Robinson N, Loveday H P, Pellowe CM, Franks PJ, Hankins M, et al. Adherence to antiretroviral therapy: Appropriate use of self-reporting in clinical practice. HIV Clin Trials. 2001;2:146-159.

122. Waite L. Does marriage matter?.Demography.1995;32(4):483-507. PMID: 8925942.

123. Monden CW. Partners in health? Exploring resemblance in health between partners in married and cohabiting couples. Sociology of Health \& Illness. 2007;29(3):391-411. http://doi.org/10.1111/j.1467-9566.2007.01003.x

124. Bauer JM, Sousa-Poza A. Impacts of informal caregiving on caregiver employment, health, and family. Journal of Population Ageing. 2015;8(3):113-145. https://doi.org/10.1007/s12062-015-9116-0

125. Larrañaga I, Martín U, Bacigalupe A, Begiristáin JM, Valderrama MJ, Arregi B. Impacto del cuidado informal en la salud y la calidad de vida de las personas cuidadoras: análisis de las desigualdades de género. Gaceta Sanitaria. 2008; 22(5):443450. https://doi.org/10.1157/13126925

126. Palomino PA, Grande ML, Linares-Abad M. La salud y sus determinantes sociales. Desigualdades y exclusión en la sociedad del siglo XXI. Revista Internacional de Sociología (RIS). 2014;72(1):45-70. doi:10.3989/ris.2013.02.16

127. Ley $8 / 2010$, de 30 de agosto, de ordenación del sistema de salud de Castilla y León. BOCYL 7-09- 2010, núm. 173, pág 14.

128. Baumann M, Lurbe K, Leandro ME, Chau N. Life satisfaction of two-year poststroke survivors: effects of socio-economic factors, motor impairment, Newcastle stroke-specific quality of life measure and World Health Organization quality of life: bref of informal caregivers in Luxembourg and a rural area in Portugal. Cerebrovasc Dis [internet]. 2012 [Citado el 1 de abril 2021];33(3):219-30. Recuperado a partir de: https://www.karger.com/Article/Pdf/333408

129. Hervás A, Cabasés J, Forcén T. Coste del cuidado informal del ictus en una población general no institucionalizada. Gac Sanit [Internet]. 2007 [citado el 1 de abril 2021];21(6):444-451. Recuperado a partir de:

https://scielo.isciii.es/pdf/gs/v21n6/originales1.pdf

130. Garrido E. Autoeficacia e intervención en problemas de familia, Psicothema [Internet]. 1993 [citado el 2 de abril de 2021];5(1):337-347. Recuperado a partir de: 
http://www.psicothema.com/pdf/1147.pdf

131. Herrera JM, Prieto C, Martin R. Las intervenciones con familias desde atención primaria de Salud por el personal sanitario. Trab Soc Salud. 2007;57: 273-282.

132. Suarez M, Alcala M. APGAR familiar: una herramienta para detectar disfunción familiar. Rev. Méd. La Paz [Internet].2014 [citado el 20 de abril de 2021];20:53-57. Recuperado a partir de: http://www.scielo.org.bo/pdf/rmcmlp/v20n1/v20n1_a10.pdf

133. Varela I, Barrón A. The influence of the couple in coping with heart disease. Acta de Investigación Psicol [Internet]. 2016;6(2):2459-2468. [citado el 3 de abril de 2021];6:2459-2468. https://doi.org/10.1016/j.aipprr.2016.06.009

134. Pastor M, López-Roig S, Rodríguez-Marín J, Salas E, Sánchez S, y Terol M. El papel del apoyo social en la experiencia de dolor crónico. Rev Psicol Soc. 1994;9(2):179191. doi: $\underline{10.1174 / 021347494763490250}$

135. Schulz R, Sherwood PR. Physical and mental health effects of family caregiving. Am J Nurs. 2008;108(9):23-7. doi: 10.1097/01.NAJ.0000336406.45248.4c

136. Davidson K, Arber S, Ginn J. Gendered meanings of care work within late life marital relationships. Can J Aging. 2000;19(4):536-55.

Doi: https://doi.org/10.1017/S071498080001250

137. Kaplan JR, Manuck SB, Clarkson TB, Lusso FM, Taub DM y Miller EW. Social stress and atherosclerosis in normocholesterolemic monkeys. Science. 1983;220(4598):733-735. doi: 10.1126/science.6836311

138. Forster A, Dickerson J, Young J, Patel A, Kalra L, Nixon J, et al. A cluster randomised controlled trial and economic evaluation of a structured training programme for caregivers of inpatients after stroke: the TRACS trial. Health Technol Assess. 2013;17(46):1-216. doi: $10.3310 /$ hta17460

139. Goodwin PJ, Leszcz M, Ennis M, Koopmans J, Vincent L, Guther H, et al. The effect of group psychosocial support on survival in metastatic breast cancer. N Engl $\mathbf{J}$ Med. 2001;345;1719-1726. https://www.nejm.org/doi/full/10.1056/NEJMoa011871

140. Martos MJ., Pozo C. y Alonso E. Influencia de las relaciones interpersonales sobre la salud y la conducta de adherencia en una muestra de pacientes crónicos. Bol Psicol [Internet]. 2008 [citado el 4 de abril de 2021];93:59-77.Recuperado a partir de: https://www.uv.es/seoane/boletin/previos/N93-4.pdf

141. House JS. Work stress and social support. Massachusetts: Addison-Wesley; 1981. $156 \mathrm{p}$.

142. Marín-Reyes F. y Rodríguez-Morán M. Apoyo familiar en el apego al tratamiento de la hipertensión arterial esencial. Salud Pública Mex [Internet]. 2001 [citado el 5 de abril de 2021];43(4):336-339. Recuperado a partir de:

http://www.scielo.org.mx/pdf/spm/v43n4/5900.pdf

143. Wang PS, Bohn RL, Knight E, Glynn RJ, Mogun H, y Avorn J. Noncompliance with antihypertensive medications. The impact of depressive symptoms and psychosocial factors. J Gen Intern Med. 2002;17(7):504-511. DOI: 10.1046/j.15251497.2002.00406.x 
144. Courneya KS, Plotnikoff RC, Hotz SB, Birkett N. Social support and the theory of planned behaviour in the exercise domain. American Journal Health Behaviour. 2000; 24(4);300-308.

145. Okun MA, Ruehkmar L, Karoly P, Lutz R, Fairholme C, Schaub R. Social support and social norms: Do both contribute to predicting leisure-time exercise?. Am $\mathrm{j}$ health behav. 2003;27(5),493-507,10.5993/AJHB.27.5.2. DOI: 10.5993/ajhb.27.5.2

146. Guzmán JM, Huenchuan S, Montes de Oca V. Redes de apoyo social de las personas mayores: marco conceptual. En: Simposio Viejos y Viejas. Participación, Ciudadanía e Inclusión Social. Santiago de Chile: 51 Congreso Internacional de Americanistas [Internet]. Santiago de Chile; 2003.

https://www.academia.edu/1914009/Redes de apoyo_social_de personas mayores m arco te $\% \mathrm{C} 3 \% \mathrm{~B} 3$ rico_conceptual

147. Ley 39/2006,14 de diciembre, de Promoción de la Autonomía Personal y atención a las personas en situación de dependencia. BOE 15-12-16, n²99.

148. Holloway M, y Fyson R. Acquired Brain Injury, Social Work and the Challenges of Personalisation. Br J Soc Work . 2016;46(5):1301-1317. DOI: 10.1093/bjsw/bcv039

149. Martínez-Vila E, Irimia P. Factores de riesgo del ictus. Anales Sistema Sanitario de Navarra [Internet]. 2000 [citado el 20 de marzo de 2021];23(3):25-31. Recuperado a partir de: https://dialnet.unirioja.es/servlet/articulo?codigo $=6604458$

150. Trevithick P. Habilidades de comunicación en intervención social. Madrid: Ed. Narcea.2002

151. Mantell A. 'Traumatic brain injury and potential safeguarding concerns' Journal of Adult Prot 2010; 12(4):31-42. https://doi.org/10.5042/jap.2010.0642

152. Headway: the brain injury association. A ticking time bomb: the false economy of cuts to brain injury support services. [Internet]. Notingham: Headwal; 2014 [citado el 20 de marzo de 2021]. 25 p. Recuperado a partir

de: https://www.headway.org.uk/media/3244/a-ticking-time-bomb-the-false-economyof-cuts-to-neurological-services.pdf

153. Higham $P$. Developing an interactive approach to social work research: The example of a research study on head injury. Br J Soc Work. 2001;31(2):197-

212. doi:10.1093/BJSW/31.2.197

154. Lawton MP, Brody EM. Assessment of older people: self-maintaining and instrumental activities of daily living. The Gerontologist 1969;9(3):179-86.doi: $\underline{10.1097 / 00006199-197005000-00029}$

155. García JV, Díaz E, Salamea A, Cabrera D, Menéndez A, Fernández-Sánchez A, et al. Evaluación de la fiabilidad y validez de una escala de valoración social en el anciano. Aten Primaria [Internet] 1999 [citado el 20 de marzo de 2021]; 23(7):434:440. Recuperado a partir de: https://www.elsevier.es/es-revista-atencion-primaria-27articulo-evaluacion-fiabilidad-validez-una-escala-14810

156. Gerencia Regional de Salud de Castilla y León. Anexo 33. Escala Gijón: Valoración SocioFamiliar. Anexo 58. Escala Abreviada de Zarit. En Cartera de Servicios de Atención Primaria. [Internet]. Valladolid: Junta de Castilla y León;2019 [citado 21 de marzo de 2021];284. Recuperado a partir de: https://www.saludcastillayleon.es/institucion/es/catalogo-prestaciones/cartera- 
servicios/cartera-servicios-atencion-primaria.ficheros/1522344cartera $\% 20 \mathrm{de} \% 20$ servicios\%202019.pdf

157. Índice de Barthel [Internet]. Wikipedia. 2021 [citado el 20 de marzo de 2021]. Recuperado a partir de: https://es.wikipedia.org/wiki/Índice_de_Barthel

158. Beaman SR de, Beaman PE, Garcia-Peña C, Villa MA, Heres J, Córdova A, et al. Validation of a Modified Version of the Mini-Mental State Examination (MMSE) in Spanish. Aging, Neuropsychol Cogn 2004;11(1):111. https://doi.org/10.1076/anec.11.1.1.29366.

159. Montaner J, Álvarez-Sabin J. La escala de ictus del National Institute of Health (NIHSS) y su adaptación al español. Neurologia. 2006;21(4):192-202.

160. Hong KS, Saver JL. Quantifying the value of stroke disability outcomes: WHO global burden of disease project disability weights for each level of the modified Rankin Scale. Stroke. 2009;40(12):3828-3833. doi: 10.1161/STROKEAHA.109.561365

161. Copstick S, Ramos SDS, Griffiths T, Wallace A. The Importance of Considering Functional Outcome and Self-awareness in the Assessment of Care Needs: Initial Evaluation of the Brain Injury Needs Indicator. Br J Soc Work 2021;00:118. https://doi.org/10.1093/bjsw/bcab026

162. Revilla L, Luna del Castillo J, Bailon, E, y Medina I. Validación del cuestionario MOS de apoyo social en Atención Primaria. Semergen: Medicina de Familia. 2005; 6(1):10-18.

163. Evans RL, Connis RT, Bishop DS, Hendricks DS, Baldwin D. Stroke: a family dilemma. Disabil Rehabil. 1994;16(3):110-118. doi: 10.3109/09638289409166287

164. Feigin VL, Forouzanfar MH, Krishnamurthi R, Mensah GA, Connor M, Bennett DA, et al. Global and regional burden of stroke during 1990-2010: findings from the Global Burden of Disease Study 2010. Lancet. 2014;383(9913):245-54. doi: 10.1016/s0140-6736(13)61953-4.

165. Carrillo Esteban J, Collado-Vázquez S. Impacto psicosocial de las enfermedades neurológicas. En: Cano la Cuerda R, Collado Vazquez S, coord.

Neurorrehabilitación métodos específicos valoración y Tratamiento. Madrid [etc.]: Médica Panamericana, 2012, p. 33-9.

166. Bausela E. Evaluación e intervención cognitivo-conductual de un enfermo mental crónico. Trab Soc Salud. 2007;57:96.

167. National Association of Social Workers (NASW). Standars for social work in health settings. En: Dubois B., Krogsrud K, editores. Social Work An Empowering Profession. Md: Nasw. 1984. p.342.

168. Institut Guttmann. Hospital de Neurorehabilitación [Internet]. Badalona: Institutt Guttmann; 2015 [citado el 22 de marzo de 2021]. Recuperado a partir de: https:/www.guttmann.com/es/criterios-priorizacion-atencion-integralneurorrehabilitadora-especializada-intensiva-institut

169. Kalra L, Evans A, Perez I, Knapp M, Swift C, Donaldson N. A randomised controlled comparison of alternative strategies in stroke care. Health Technol Assess. 2005;9(18):1-79. doi: 10.3310/hta9180 
170. Torres S, Carpallo M, Serrat LL, Salto I, Granados MA, Alemamy L. Estudio protocolarizado de la eficacia de la coordinación de trabajo social entre niveles asistenciales sanitarios. Trab Soc Salud. 2006;54:193-200

171. Aranda A, Royo L. Estudios de los cambios en el funcionamiento psicosocial de los usuarios con trastorno mental grave del centro de rehabilitación psicosocial de Asapme. Trab Soc Salud. 2016;85:51-56

172. Lara L, Díaz M, Herrera E, Silveira P. Síndrome del "Cuidador" en una población atendida por equipo multidisciplinario de atención geriátrica. Rev Cubana Enferm [Internet]. 2001 [citado el 22 de marzo de 2021];17(2):107-111. Recuperado a partir de: http://scielo.sld.cu/pdf/enf/v17n2/enf07201.pdf

173. Ituarte A. Trabajo Social Clínico en el contexto sanitario: Teoría y Práctica. Trab Soc Salud. 2011;68:310.

174. Caballero ME. La continuidad asistencial en la práctica profesional. Trab Soc Salud. 2006;54:58.

175. Garcia M, Gonzalo L, Bayona N. La especialización en trabajo social: Un camino a la experiencia. Trab Soc Salud. 2007;57:25.

176. Ue B. Social work and the stroke patient. Clin Orthop Relat Res. 1978;(131):101103.

177. Moros JS, Ballero F, Jáuregui S, Carroza MP. ANALES Sistema Sanitario Navarra [Internet] 2000 [citado el 22 de marzo de 2021];23(3):173-180. Recuperado a partir de: file:///C:/Users/Lenovo/Downloads/6745-Texto\%20del\%20artículo-11740-110-20090626.pdf

178. Ley Orgánica 3/2018, de 5 de diciembre, de Protección de Datos Personales y garantía de los derechos digitales. BOE 6-12-2018, núm 294.

179. Arredondo R, Cosano F. Diccionario de trabajo social. Málaga: Colegio Oficial de diplomados en Trabajo Social de Málaga; 2013. p. 17.

180. López F, Portilla JC, Leno C, Párraga JM, Gamez-Leyva G, Casado Naranjo I. Diferencias de género en la calidad de vida a largo plazo tras un ictus: influencia del estado funcional y el estado de ánimo. Neurologia. 2020;35:470-

8. doi.org/10.1016/j.nrl.2017.10.002

181. Martin-Schild S, Albright KC, Tanksley J, Pandav V, Jones EB, Grotta JC, et al. Zero on the NIHSS does not equal the absence of stroke. Ann Emerg Med.

2011;57(1):42-5. doi: 10.1016/j.annemergmed.2010.06.564

182. Woldag H, Gerhold LL, de Groot M, Wohlfart K, Wagner A, Hummelsheim H. Early prediction of functional outcome after stroke. Brain Injury. 2006;20(10):1047-52. https://doi.org/10.1080/02699050600915422

183. Grupo Neuro Ictus. Escala NIHSS National Institute of Health Stroke Score. Sociedad Española de Medicina de Urgencias y Emergencias [Internet]. Recuperado a partir de: https://www.tiempoescerebro.com/wp-content/uploads/2017/06/nihss.pdf

184. Van Swieten JC, Koudstaal PJ, Visser MC, Schouten HJ, van Gijn J. Interobserver agreement for the assessment of handicap in stroke patients. Stroke. 1988;19(5):6047. doi: 10.1161/01.str.19.5.604. 
185. Raymond SK, Joanna OY, Cheng E, Wong W, Lawrence KS, Wong JW, Kwok T. Handicap and Its Determinants of Change in Stroke Survivors. Stroke. 2008;39(1):14853. doi: 10.1161/STROKEAHA.107.491399

186. González JG, Palacios E, García AS, González DC, Caicoya AM, Sánchez AF, et al. Evaluación de la fiabilidad y validez de una escala de valoración social en el anciano. Aten Primaria. 1999;23:434-40

187. Mahoney FI, Barthel D. Functional evaluation: The Barthel Index. Maryland State Medical Journal [Internet]. 1965 [Citado el 4 de abril de 2021];14:61-5. Recuperado a partir de: https://pubmed.ncbi.nlm.nih.gov/14258950/

188. Duffy L, Gajree S, Langhorne P, Stott DJ, Quinn TJ. Reliability (inter-rater agreement) of the Barthel Index for assessment of stroke survivors: systematic review and meta-analysis. Stroke. 2013;44(2):462-8. Doi: 10.1161/STROKEAHA.112.678615

189. Hunter JD. Matplotlib: A 2D Graphics Environment. Comput Sci Eng. 2007; 90:95-9. Doi: 10.1109/MCSE.2007.55

190. McKinney W. Data Structures for Statistical Computing in Python. Proceedings of the 9th Python in Science Conference. 2010;1(Scipy):56-61. Doi: 10.25080/majora92bf1922-00a

191. Seabold S, Perktold J. Statsmodels: Econometric and statistical modeling with python. Proceedings of the 9th Python in Science Conference. 2010;57-

61. Doi:10.25080/MAJORA-92BF1922-011

192. Pedregosa F, Varoquaux G, Gramfort A, Michel V, Thirion B, Grisel O, Blondel M, Prettenhofer P, Weiss R et al. Scikit-learn: Machine Learning in Python. J Mach Learn Res. [Internet]. 2011 [citado el 6 de abril de 2021];12:2825-2830. Recuperado a partir de: http://www.jmlr.org/papers/volume12/pedregosa11a/pedregosa11a.pdf

193. The jamovi project. Version 1.0 [Computer Software]. [Internet] 2019 [citado 8 de abril de 2021. Recuperado a partir de: https://www.jamovi.org

194. Vega Valdés P, Murias Quintana E, Morales Santos E, Cigarrán Sexto H, Larrosa Campo D, Benavente Fernández L, et al. Análisis de los costes directos asociados a la trombectomía mecánica y a la fibrinólisis intravenosa en el Hospital Universitario Central de Asturias. Rev Neurol. 2018;66(01):7. doi: 10.33588/rn.6601.2017208

195. Sánchez M, Pomar Fernández FJ (Dir). Las necesidades sociales originadas a consecuencia de la enfermedad en pacientes adultos hospitalizados [tesis en internet]. [Madrid]: Universidad Complutense de Madrid; 2016 [citado el 4 de mayo de 2021]. Recuperado a partir de: https://eprints.ucm.es/id/eprint/36980/1/T37009.pdf

196. Baztán JJ, Pérez Martínez DA, Fernández-Alonso M, Aguado-Ortego R, Bellando-Alvárez G, de la Fuente-González AM. (2007). Factores pronósticos de recuperación funcional en pacientes muy ancianos con ictus. Estudio de seguimiento al año. Rev Neurol. 2007;44(10):577-583.

doi: https://doi.org/10.33588/rn.4410.2006521

197. Fernández A, Ruíz J, Tejada H, Marta J. Validación de la versión en español del cuestionario telefónico modificado simplificado Rankin Scale. Neurología [Internet]. 2019 [citado el 10 de mayo de 2021];S0213-4853(19)30047-7. doi: $\underline{10.1016 / j . n r l .2019 .03 .003}$ 
198. Peñalver Pérez F, López Barrio M J, Marín López T, Saorín Marín D. El ictus en las mujeres: perspectiva de género en el ictus cardioembólico secundario a fibrilación auricular. Revista Ene Enfermería. [Internet]. 2015 [citado el 4 de mayo de 2021]; 9(2). Disponible en: http://ene-enfermeria.org/ojs/index.php/ENE/article/view/497

199. Ley 14/86, de 25 de abril, General de Sanidad. BOE 29-04-1986, núm. 102, pág. 12.

200. Legg $L$, Langhorne $P$. Rehabilitation therapy services for stroke patients living at home: systematic review of randomised trials. Lancet. 2004;363(9406):352-356. doi:10.1016/S0140-6736(04)15434-2

201. Green J, Young J, Forster A, Collen F, Wade D. Combined analysis of two randomized trials of community physiotherapy for patients more than one year post stroke. Clint Rehabil. 2004;18(3):249-252. doi:10.1191/0269215504cr747oa

202. Banks JL, Marotta CA. Outcomes validity and reliability of the modified rankin scale: Implications for stroke clinical trials - A literature review and synthesis. Stroke. 2007;38(3):1091-6. doi: 10.1161/01.STR.0000258355.23810.c6. 
ANEXOS 


\title{
ANEXO 1: PUBLICACIONES DERIVADAS DE LA TESIS
}

\section{PRIMER ARTICULO “ANÁLISIS DE LOS FACTORES ASOCIADOS A LA RECUPERACIÓN INTEGRAL DE PACIENTES DE ICTUS ALTA HOSPITAL DE AGUDOS”}

\section{AN.̇LISIS DE FACTORES ASOCLADOS A LA RECUPERACIÓN INTEGRAL.} DE PACIENTES DE ICTUS AL_ALTA DE HOSPTTAL DE AGUDOS

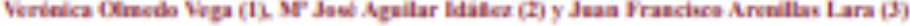

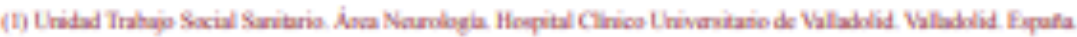

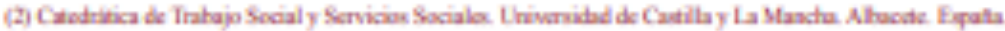

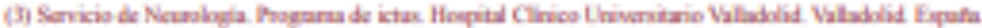

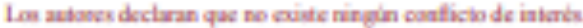

\section{RESUMEN}

Fundamentes: El ictus es un problema de Salud Pullica de peimera meznitud, siendo la primera causa de discapacidad en Espella A pesar de que se conoce et papel fundumental de la rehabilinación psicotisica en los pacieates que sutrea esta patolozia poco se conoce sobet la recuperacila integral y tuncional de este tipo de pocientes. L.as Sistemas Púbicos de Salud eoriean o gestionan parte del tratamieato, pero aunea su iocalidad. El objetivo de nuestro estudio fue identificar las factores que diffeultan el acceso a bos diferentes tratamienbos y cuidados que peneniten la resuperacala funciceal del paciente coe ictus.

Métodos: Se realizó andlisis descripeino correlacional bivariable de una muestra de 102 pacienies con ictux, mayces de 30 anck, ingresados en el Hospital Clinico Universitane de Valladoldd, que precisaban wa rehubilitación integral al ala hospinalaria. Adicionalmente, se realizs wa anilisis multivariable (regresiòn bogistica) para ajustar resultados y elininar variables confuseras

Resultados: El estudio evidencib que los tacweres inicialmente wociados al seceso a la rehabilitacice sotal fuesce la edad ( $\mathrm{p}<0.001)$, el lugar de resadencia $(\rho<0,001)$ el estado civil $(p=0,007)$ y la existencia de apoyo familiar ( $\mathrm{p}=0,01)$. E sero $(\mathrm{p}=0,18)$ y las ingrescis ecoolmicos $(\mathrm{p}=0.62)$ no petsentarce asociacion estadbsticantente significativa. La edad y el lugar de residencia (nural o urtano) fueron los factores explicativos del aceeso a la rellabilitacibe integral (se obtuvierce valores ajustados al resto de variables de pac0,001).

Conclusiones: Los resultados muestran la exiciencia de variables de acceso muy desigual a la rehubi. litacioln integral, coe las consecuencias de perduda de calidad de vida $\mathrm{e}$ imposibilidad de recuperacice fiecional que ello imelioa, tanto para pocientes de ictus de edad avanoada como para pacientes que residen en el medio nural.

Palabras clave: letux, Neurologia, Estade de salud, Caldad de vida, Apoyo social, Astencibe integral de salud. Bienestre social, Factoes sociociondenicos, Accesibilidad a los servicios de salud.

Comerpondencia

Verima Clindo Voes

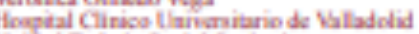

Inidad Trabajo Sacial Sarizariz

Q Ramia y Cajal, wh

4000 valiadatid Frnana

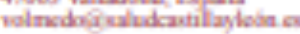

\section{ABSTRACT}

Analysis of factors associated

with the comprehensive recevery of streke

patients at acute hospital discharge

Background: Suroke is a public health problem of the first magnitude, being the first cause of disability in Spain. Ailtough the findumental role of poychoplysical rehubilinatice is knowa in patients suffering thoen this pathology, little is baown about the integal and functiceal recovery of this type of patient. The Public Health Systems pay for of maneope part of the treatment, but never is enticty. The aim of our study was to ideneify the factors that hinder access to the differeat treatments and care that allow the functional recovery of stroke patieats.

Methods: Bivariate coerelational descriptive analysis was perfoemed on a sample of 102 staoke potients, over 30 yeurs of age, admitted to the University Clinical Hospinal of Valladolid, who required coesprehersive schabilitation upon discharge from hospital. Addriceally, a multivanable analysis (logistic regressice) was carried out to adjust resulss and eliminate confusing variables

Results: The stady showed that the facsors initielly associated with access bo sotal rehahilination were age ( $\mathrm{p}<0.001)$, place of residence (p $<0.001)$, marial status $(p=0.007)$ and the existence of tamily support $(p=0.01)$. Ser $(p=0.18)$ and income $(p=0.62)$ were not statistically sighificam. Age and place of residence $(\mathrm{ru}$. ral or urban) were the explanabory factors for access to comerchensive rehabilnatioe (values adjussed foc all other variables pa0.001 were obtained).

Conclusions: The results show the existence of very unequal access variables to comprehensive rehebilitation, with the consequences of loss of quality of life and impossibility of functioned recovery that this implies, both for stroke patients of advanced age and for patients residing in nural areas.

Key words: Stroke, Neurobogy, Health staous, Quality of life, Social support, Comprellensive health cart Social welfare, Socioeconomic factors. Health services accessibility.

Cra mecrida: Otnodo Vega V, Apular ldifece MU, Arcrilla

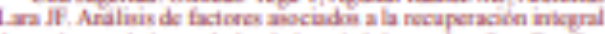
de pacianke de icha al alta de hospizal de aradoe. Rev Exp Sa.

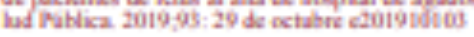




\section{INTRODUCCIÓN}

El ictus (tanto isquémico como hemorri. gico) es la primera causa de discapacidad en adultos ${ }^{10}$. Dicha discapacidad suele deberse al amplio y heterogéneo número de secuelas que provoca ${ }^{23}$, afectando de forma muy importante la vida cotidiana, al alterar de forma diversa y compleja las funciones cognitivas y funciona$\operatorname{les}^{23}$. L.as seis áreas que se ven afectadas por el ictus $^{60}$ (cognitiva, emocional, comunicación y lenguaje, motora, sensitiva y visual) sufren importantes efectos que interactuan y retroactuan unos con otros, generando déficits complejos que van mis allá de cada área de función. La rehabilitación en el accidente cerebrovascular (ACV) es un proceso que, con un enfoque integral $e$ interdisciplinario, busca disminuir b incidencia de complicaciones que son preveni. bles, evitar un aumento del déficit neurológico, disminuir los tiempos de hospitalización, apoyar $e$ integrar al paciente $y$ a su familia en el proceso de tratamiento, y optimizar los resulta. dos funcionales y la reintegración social ${ }^{\text {t5 }}$.

La rehabilitaciớn integral se caracteriza por su globalidad, pues trabaja aspectos fisicos, cognitives y sociales, con el fin de conseguir la mixima independencia posible, promover la salud y mejorar la calidad de vida del pacien. te. En el caso de los pacientes con daño cere. brovascular, se trata de un proceso limitado en el tiempo y orientado a compensar los déficits. minimizando la discapacidad asi como maximizando la capacidad funcional, la independencia del paciente y la reintegración en su entorno. Eistos objetivos requieren de un abordaje multidisciplinar del proceso rehabilitador ${ }^{\text {en }}$.

Los criterios de rehabilitación integral los establece el equipo responsable del proceso asistencial, que está integrado por diferentes profesionales competentes en su airea de intervención, aunque muchos otros profesiona. les intervengan lo largo de todo el procesd?
En el Hospital Clinico Universitario de Valladolid (HCUV) se definen los criterios y se valoran los avances a través de reuniones semanales (convocadas por la trabajadora social del arrea de neurologia) del equipo multidisciplinar que interviene con el paciente. Este equipo está compuesto por una trabajadora Social, un neurólogo, un logopecta, un fissoterapeuta, un miembro de Enfermeria y un rehabilitador. Se analizan las áreas afectadas en el paciente, el pronóstico, y la situación personal y sociofamiliar, estableciéndose el plan de rehabilitaciớn integral al alta bospitalaria.

Este plan incluye:

i) Äreas afectadas.

ii) Indicaciones del tratamiento que precisa al alta por parte de cada profesional competente en ese írea afectada.

iii) Pronóstico.

iv) Caracteristicas personales.

v) Sítuación sociofamilar del paciente.

L.a inclusión de estos cinco componentes garantiza que acceda a ese tratamiento rehabilitador integral personalizado.

El concepto de discapacidad es multidimensional, abarcando no solo la dimensión social o la biológica. Por lo tanto, seria inadecuado pensar en la rehabilitación desde una visión única, entendida esta como una respuesta a la inclu= sión de la persona en su medio. Se basa en la capacidad y experiencia de un equipo interdis: ciplinar, que aborda la asistencia del paciente de manera integral, contempla todas las áreas afectadas por el paciente con ictus, buscando en todo momento la implicación y participaciớn activa del paciente y de las familias en el proceso rehabilitador. Se plantea por este motivo una 
rehabilitación integral que asegure la mejor recuperación funcional posible del paciente.

En el acceso a la recuperación funcional del paciente, factores como la edad, el sexo, el estado civil, el lugar de residencia (rural/urbano), los ingresos económicos, o la existencia de apoyo familiar, podrian estar asociados con la posabilidad de recibir una rehabilitación integral.

Es una realidad que no existen centros de recuperación integral, ni públicos ni privados. en todos los municipios donde residen los pacientes que sufren ictus. En la mayoria de los casos, los que existen están situados en otra localidad distinta $y$, con frecuencia, están lejos de su residencia habitual.

Por ello, es imprescindible conocer y abordar los factores que pudieran condicionar el acceso a la rehabilitación, en contextos en los que no existen centros especificos para ello (ni van a existir). Investigaciones recientes ${ }^{(1)}$ parecen evidenciar que el sexo en si mismo (independientemente de la edad, el estatus funcional previo, las comorbilidades y otros factores cardiovasculares) puede afectar en cómo las mujeres y los hombres padecen el ictus. El sexo influye en la posibilidad de participar en un tratamiento rehabilitador, porque las cargas familiares y profesionales entre hombres y mujeres no son equitativas, $y$ las responsabilidades que cada uno tiene im: plican tiempo y dedicación. Esto provoca que las mujeres no tengan la misma disponibilidad de tiempo que los hombres para realizar un tratamiento rehabilitador. La edad condiciona el acceso a la rehabilitación integral por motivos que impiden adaptarse o seguir el tratamiento rehabilitador pautado. Las personas mayores de 65 años presentan un declive de todas aquellas estructuras que se habian desarrollado en las etapas anteriores, lo que ocasiona limitaciones funcionales en la persona: dificultad para conducir, necesidad de supervisión continua, o uso de ayudas técnicas para los desplazamientos. Además, algunos tratamientos rehabilitadores tienen como criterio de exclusiỏn la edad cronológica.

E1 estado civil se asocia a determinados estados emocionales y a la percepción de sentimientos de soledad, afecto, companilia y apoyo. Influye sobre la salud emocional y mental de las personas $5^{10} \mathrm{y}$, por tanto, en la predisposición a colaborar del paciente en sa propio proceso de rehabilitación. Respecto a la situación económica, el padecimiento de una enfermedad, ademis del coste personal, familiar y humano que entraña, también conlleva un coste económico considerable, en cuanto a adaptación de la vivienda o pago de tratamientos rehabilitadores no subvencionados, entre otros.

E1 lugar de residencia, la situación geograifica y d medio urbano o rural suponen un gran hándicap en algunas situaciones. Muchas veces, se asocia la vida en el campo a la tranquilidad y la calidad de vida, pero cuando las personas sufren un ictus necesitan tratamientos y servicios rehabilitadores no disponibles en su bocalidad. El apoyo familiar en una situaciôn de pérdida o disminución funcional de la aur tonomia de un paciente, como consecuencia de los déficits neurológicos residuales tras un ictus, implica un proceso de adaptación tanto en el paciente como en su familia. El impacto del ictus provoca, en muchos casos, la no aceptación de la realidad y la desestabilización o la desunión familiar, pues no deja indiferente a la familia, teniendo entonces que asumir nuevos roles y una nueva dinámica familiar ${ }^{14}$. La in fluencia familiar en el tratamiento rehabilitador reviste gran importancia debido a las limitaciones de la persona (fisicas y psiquicas) ante la aparición de un ictus, lo que hace que cobre todavia mayor importancia el papel de la familia en el acceso, mantenimiento y apoyo al tratamiento rehabilitador del paciente, formando parte activa de ese proceso. 
La identificación de la posible influencia de algunos de los factores mencionados permite introducir medidas que garanticen la recupera. ción funcional del paciente.

El objetivo de este estudio fue determinar en qué medida ciertas caracteristicas personales y sociofamiliares de las personas que sufrian ictus ingresadas en un hospital de agudos estu. vieron asociades al acceso a una rehabilitación integral para la recuperación total del mismo.

\section{SUJETOS Y MÉTODOS}

Se realizó un estudio exploratorio descripti. vo correlacional multivariable, de una muestra de 102 pacientes de ictus (sobre una población de 490) ingresados entre septiembre de 2016 y noviembre de 2017 en el Hospital Clínico Universitario de Valladolid (HCUV). Eran susceptibles de rehabilitaciôn integral, fueron seleccionados por orden y fecha de ingreso, entre el 1 de septiembre de 2016 y el 20 de enero de 2017). El nivel de confianza fue del $95 \%$.

El Sistema de Salud de Castilla y Leổn está constituido por 11 Ảreas de Salud. El Área de Salud Este de Valladolid cuenta con dos hospitales: el HCUV y el de Medina del Campo. El HCUV es un centro terciario, siendo referencia en cuanto a unidad de ictus, por lo que pacien: tes que sufren un ictus en otras provincias de Castilla y Leoón acuden esta unidad.

Los criterios de inclusiốn seleccionados fueron: pacientes mayores de 30 años que habian sufrido ictus, pertenecientes al Ȧrea de Salud Este $y$ al HCUV, ingresados en el Servicio de Neurologia del HCUV, que bubieran sobrevivido, que precisaran y fueran capaces al alta de seguir un tratamicnto rehabilitador integral para la recuperación de secuelas. Esto suponia un estado neurológico estabilirado con déficit significativo en al menos 2 áreas, con capacidad cognitiva que le posibilitara seguir instrucciones $y$ capacidad fisica para tolerar un programa de terapia activa. Ademis, el enfermo debia estar en condiciones fisicas que le permitieran efectuar y/o tolerar la movilizaciōn, y con condiciones mentales que le capacitaran para una comprensión y colaboración adecuadas.

L.a necesidad de un tratamiento rehabilita= dor integral se decidia en las reuniones semanales del equipo multidisciplinar que atiende al paciente neurológico durante su periodo de bospitaliración en el servicio médico de neurologia. En estas reuniones eran analizados y deliberados diversos aspectos por parte de los diferentes profesionales del equipo interdisciplinar de neurologia:

- Ảreas afectadas: lenguaje, deglución, motora, cognitiva, movimiento, emocional.

- Discapacidad: Escala Ranking.

- Gravedad clinica: Escala NIHSS.

- Pronóstico de recuperación.

Se informaba de la evolución clinico-social del paciente durante su ingreso, su pronóstico y el tratamiento rehabilitador que precisaba. La trabajadora social administraba el indice de Katz de independencia en las actividades básicas de la vida diaria, $y$ para el estudio $y$ valoración de la situación sociofamiliar del paciente aplicaba la Escala Apgar Familiar.

Se diseñaba y era establecido por el equipo un plan individualizado de rehabilitación integral post-hospitalización, custodiado por el trabajador social de neurologia, dỏnde se recogia qué tratamiento rehabilitador precisaba el paciente (por ejemplo: logopedia + fisioterapia + neuropsicologia), el pronóstico de tiempo, los tratamientos cubiertos $y$ no cubiertos por el sistema público de salud, $\mathrm{y}$ la colaboración del paciente y la familia en la rehabilitación que precisaba. 
Los 102 pacientes seleccionados eran consecutivos, cumplian los criterios descritos y presentaban afectación en al menos dos dominios que les ocasionaban dependencia funcional.

Los criterios de exclusión seleccionados fue: ron: pacientes menores de 30 años, o que no ha: bian sobrevivido, con deterioro cognitivo que les impidiese responder al tratamiento rehabilitador $\mathrm{y}$ pacientes en situación paliativa o severamente dañados que no se beneficiaban de rehabilitación activa, asi como aquellos sin secuelas.

Las variables independientes fueron: edad, sexo, estado civil, ingresos mensuales, lugar de residencia habitual y apoyo familiar (determinado seguin Apgar familiar: positivo si la funciớn familiar era normal o presentaba disfunciốn leve, y negativo si la disfunción familiar era moderada o severa).

La variable dependiente fue el acceso a la rehabilitación integral, entendida como aquella que contemplaba la administración de tratamientos de rehabilitación precisados en todas las aireas afectadas (lenguaje, fisica, cognitiva, vistal), dando respuesta a la globalicad de la persona. Para la comprobación de esta varia. ble, se accedió al informe clinico del paciente que señalaba su juicio clinico, áreas afectadas. tratamiento preciso, dictamen de valoración del equipo clinico especializado y la entrevista de seguimiento del tratamiento rehabilitador integral con el paciente. El periodo de rehabilitación integral de los pacientes que accedieron a la misma osciló entre 4 y 12 meses, desde el alta hospitalaria.

El análisis de datos fue inicialmente de tipo descriptivo y correlacional bivariable, mediante test chi-cuadrado para valores categóricos y tests t-student y MWU para valores numéricos. En segundo lugar, se realizo un anali. sis multivariable mediante regresiôn logistica, a fin de eliminar posibles variables confusoras. Teniendo en cuenta que el estado civil y el apoyo familiar podian ser variables confusoras asociadas a la edad y al lugar de residencia, se realizó una regresión logistica, tanto de manera individual como junto con el resto de variables independientes, a fin de ajustar los resultados y poder determinar qué factores eran realmente explicativos del acceso a la rehabilitación integral.

L.as tres fuentes de información sobre la evolución y el resultado del tratamiento de rehabilitación integral post-alta bospitalaria del paciente fueron:

- Consulta de seguimiento con el especialis. ta en neurologia entre los $6-9$ meses desde el alta hospitalaria. Se midió el resultado funcional tras el ictus aplicando la escala Ranking y el İndice Barthel.

- Entrevista presencial con un trabajador social sanitario de neurologia a los 6-9 meses del alta hospitalaria, para conocimiento y valoracỉón de la situación socio-familiar y laboral: incorporación a la vida laboral, apoyo familiar, actividades que realizaba, tareas a las que habia tenido que recharar por imposibilidad de realización, entre otras (se aplicó Escala Apgar Familiar e İndice Katz).

- Informes al alta de los centros o servicios dónde estaba recibiendo el tratamiento rehabilitador.

- Entrevista al mes de la finalización del tratamiento rehabilitador con neurologia y trabajo social sanitario para constatar la situación clinico-social del paciente.

E. análisis estadistico se realizó con el software Python 3.7, utilizando las librerias Matplotlib $^{(i)}$, Pandas ${ }^{15}$, Statsmodels ${ }^{15}$, ScikitLearn $^{16}$ y Jamovir ${ }^{19}$. 


\section{RESULTADOS}

Resultados descriptivos de la muestra analizada. En cuanto a variables independientes, fueron: por edad, un $19 \%$ tenia menos de 60 años, el $75 \%$ tenia entre 60 y 90 años, y un $6 \%$ más de 90 . El $54 \%$ eran hombres y el $46 \%$ mujeres. Un $55 \%$ eran personas casadas, un $27 \%$ viudas y un $18 \%$ estaban solteras. El $56 \%$ vivia en zona urbana y el $44 \%$ en zona rural. El $59 \%$ tenia apoyo familiar y un $41 \%$ carecia del mismo. En cuanto a la sítuación económica, un $20,6 \%$ tenia ingresos mensuales inferiores a 500 euros. un $32,4 \%$ tenia entre 500 y 1.000 euros, $y$ un $47 \%$ ingresaba mis de 1.000 euros.

Respecto a la variable dependiente, un $53,9 \%$ recibió rehabilitación integral, es decir, el tratamiento requerido segün todas las áreas afectadas, tanto desde los servicios püblicos (fisioterapia, logopedia y atención social) como a nivel privado (neuropsicologia, terapia ocupacional). E. $46,1 \%$ restante, a pesar de ser susceptible de rehabilitación integral, no pudo acceder a la misma.
Resultados del análisis de correlación. Aplicando el test chi-cuadrado de Pearson mostraron evidencias significativas de asociación varios factores con el acceso a la rehabilitación integral (p-valor 00,05 ), mientras que otros factores no presentaron una asociación estadisticamente significativa ( $\mathrm{p}$-valor $>0,05$ ).

Como se muestra en la tabla 1 , fueron cuatro los factores asociados al acceso a la rehabi. litación integral: el lugar de residencia (medio rural/medio urbano), el estado civil, la edad y la existencia de apoyo familiar. Otros factores, como los ingresos económicos o el sexo, no presentaron asociación significativa.

E. lugar de residencia se evidenció como un factor claramente asociado a la rehabilitación integral ( $\mathrm{p}-0,001)$, que condicionaba fuertemente el acceso a la misma, tal como muestra la figura 1. La gran mayoria de los pacientes que accedieron a rehabilitación integral vivian en zona urbana (exactamente un $68,4 \%$ ), y la mayoria de quienes vivian en zona rural no accedieron a la misma (un $64,4 \%$ ).

\begin{tabular}{|c|c|c|c|c|}
\hline \multicolumn{5}{|c|}{$\begin{array}{l}\text { Tabla } 1 \\
\text { Rehabilitación según variables basales. }\end{array}$} \\
\hline \multicolumn{2}{|c|}{ Variables basales } & Rehabilitación Si & Rehabilitación No & $\begin{array}{c}\text { P-valor } \\
\text { (chi-cuadrado) }\end{array}$ \\
\hline \multirow{2}{*}{ Residencia } & Urbana" & $39(38 \%)$ & $18(18 \%)$ & \multirow{2}{*}{$<0,001$} \\
\hline & Rurall? & $16(16 \%)$ & $29(28 \%)$ & \\
\hline \multirow{2}{*}{ Sexo } & Masculino & $33(32 \%)$ & $22(22 \%)$ & \multirow{2}{*}{0,18} \\
\hline & Femenino & $22(22 \%)$ & $25(25 \%)$ & \\
\hline \multirow{3}{*}{ Estade civil } & Casade't & $32(31 \%)$ & $24(24 \%)$ & \multirow{3}{*}{0,007} \\
\hline & Soltero" & $14(14 \%)$ & $4(4 \%)$ & \\
\hline & Viude' & $9(9 \%)$ & $19(19 \%)$ & \\
\hline \multicolumn{2}{|l|}{ Edad (media) } & 66,2 & 81,6 & $<0,001$ \\
\hline \multicolumn{2}{|c|}{ Ingreses mesuales (media) } & $856 t$ & 9016 & 0,62 \\
\hline \multirow{2}{*}{ Apoye familiar } & $\sin ^{*}$ & $26(25 \%)$ & $34(33 \%)$ & \multirow{2}{*}{0,01} \\
\hline & Now? & $29(28 \%)$ & $13(13 \%)$ & \\
\hline
\end{tabular}




\section{Figura 1}

Acceso a la rehabilitación integral según tipo de localidad de residencia.

NOREHABILITACIÓN INTEGRAL III SI REHABILITACIÓN INTEGRAL

$64,4 \%$

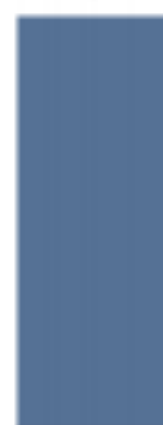

Rural
$68,4 \%$

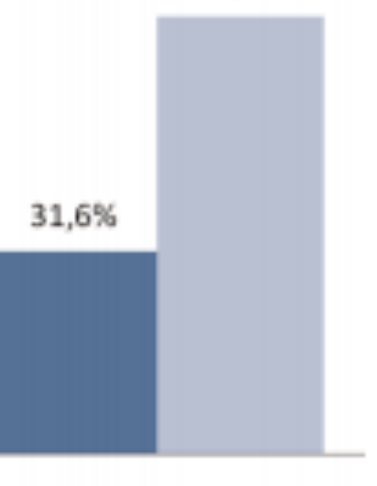

Urbana
Como se evidencia en la figura 2, la edad se mostró como un factor muy claramente asociado a la posibilidad de recibir rehabilitación integral, ya que a mayor edad se reducian considerable y muy significativamente las posibilidades de acceso a una rehabilitación integral ( $p<0,0001$ ). La edad media de quienes accedieron a la rehabilitación integral era de 66,2 años, y la de quienes no accedieron era de 81,7 años. Si comparamos las medias de edad entre quienes recibieron o no rehabilitación integral, tanto el test t-student ( $p \subset 0,0001)$ como el test Mann-Whitney $U(p<0,001)$ arrojaron la misma conclusión. La única variable numérica de interés resultó ser la edad. Se aplicaron tres tests de normalidad a dicha variable (Anderson, Kolmogorov-Smirnov y Saphiro-Wilk). Seguin el test de Saphiro-Wilk, la distribución no era normal, mientras que segủn el test de Kolmogorov-Smirnov y el test de Anderson si que era normal. Observando el gráfico de distribución de la edad de la figura 3, vemos una distribución que se aproximaba a la normal, mientras que si se analizan los gráficos $\mathrm{q}-\mathrm{q}$, la distribución no parecia normal. Por estos motivos, se realizaron los tests paramétricos y no-paramétricos. Los tests de normalidad se tendrain que volver a repetir en caso de replicar el análisis con más datos o datos diferentes.

El estado civil fue el tercer factor que presentó diferencias significativas en el acceso a la rehabilitación integral ( $p-0,008)$, siendo mayor el porcentaje de personas solieras que la lograhan $(77,8 \%)$, frente un $57,1 \%$ de casadas y un $32,1 \%$ de viudas. Ahora bien, analirando conjuntamente la edad y el estado civil (figura 3), y consideran= do que la edad media de las personas viudas era de 81,4 años, la de las casadas de 74,4 años $y$ la de las solteras de 57,3 años, para determinar si el estado civil podria ser un factor de confusiốn al estar en si mismo correlacicnado con la edad, se realizó tanto un test ANOVA como un KrusikalWallis, para evaluar la diferencia de medias de edad entre los tres grupos. Ambos fueron signifi. cativos ( $p q 0,0001$ ), por lo que podemos concluir que era un factor de confusión altamente correlacionado con la edad (ver figura 4). 
Figura 2

Distribución del acceso a la rehabilitación integral por edad.

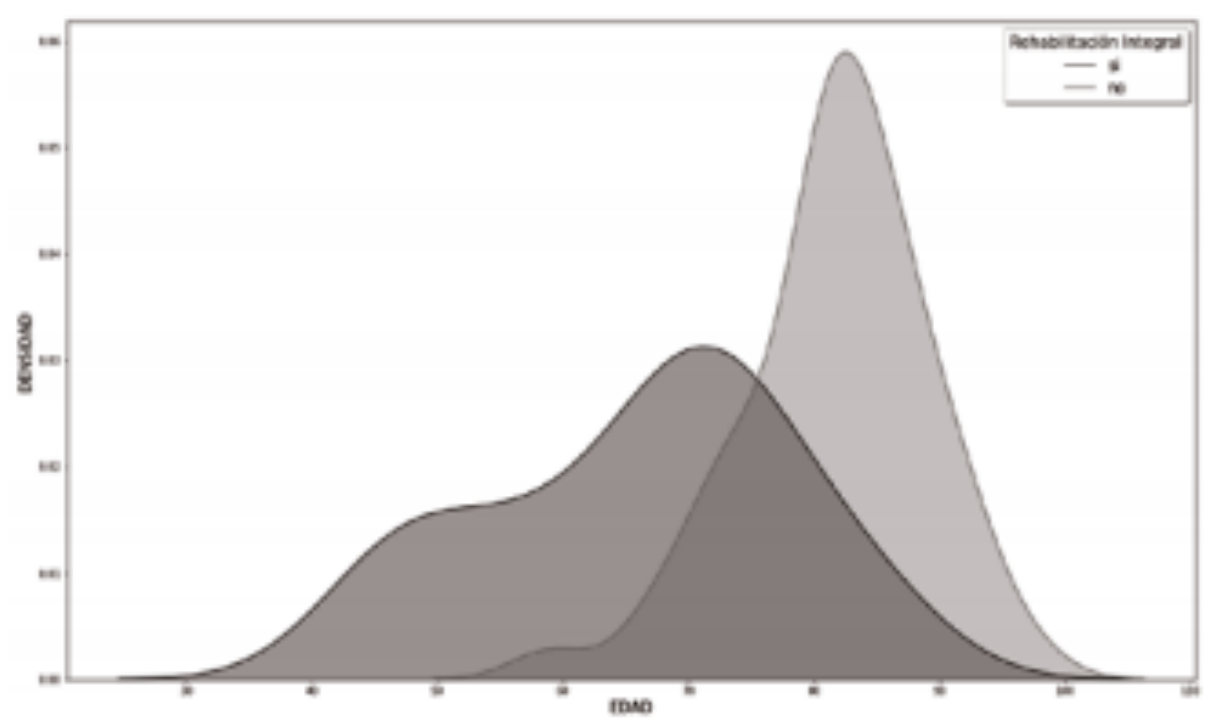

Figura 3

Distribución por edad.

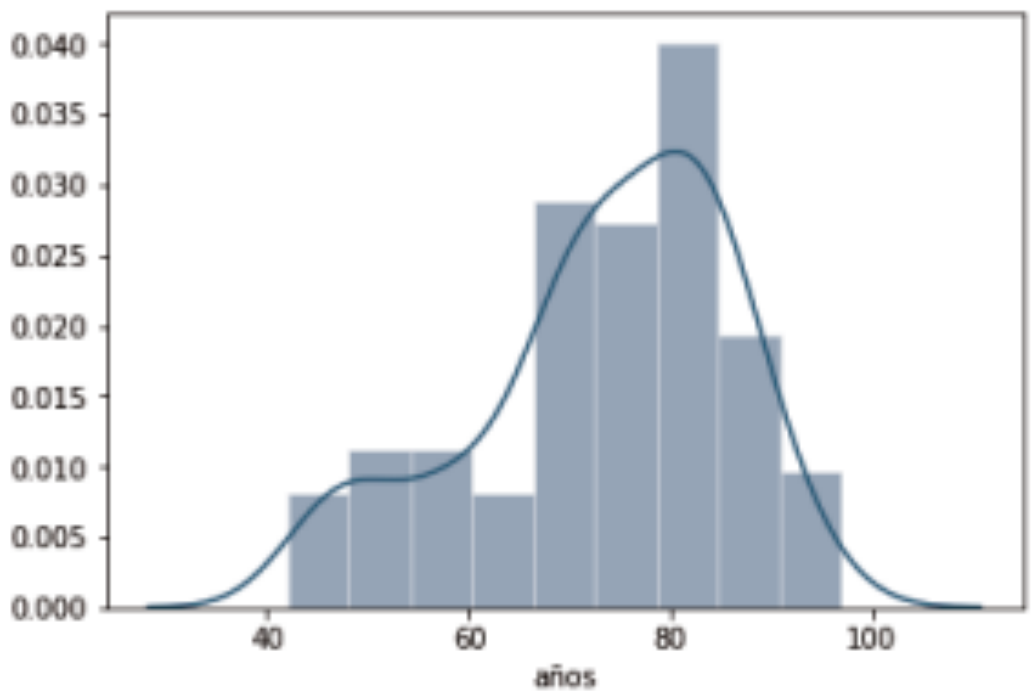


Figura 4

Distribución del acceso a la rehabilitación integral por edad.

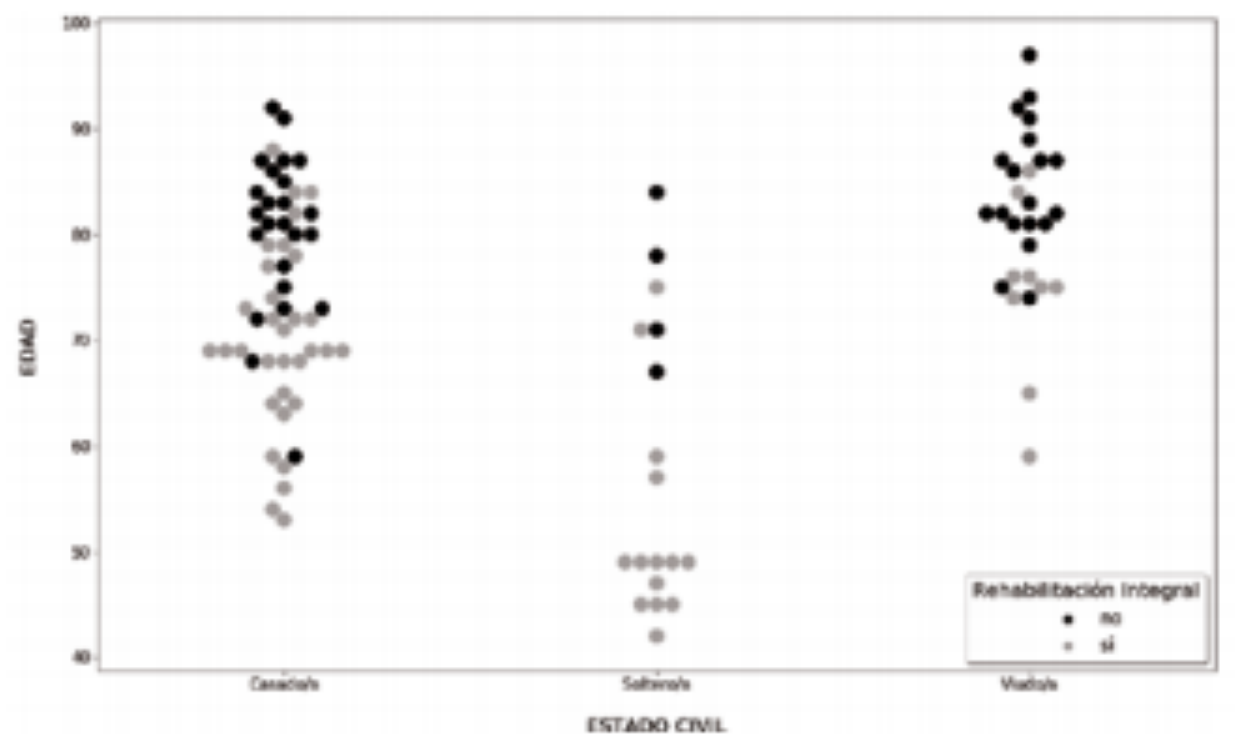

Un cuarto factor asociado al acceso a la re: habilitación integral fue el apoyo familiar $(\mathrm{p}-0,018)$. En la tabla 2 se muestra que el apoyo familiar se asoció en mayor medida a zoma rural que a zona urbana.

\begin{tabular}{|c|c|c|}
\hline \multicolumn{3}{|c|}{$\begin{array}{c}\text { Tabla } 2 \\
\text { Existencia de apoyo familiar } \\
\text { seguin tipo de localidad ( } \mathrm{p}-\mathbf{0 , 0 0 1}) \text {. }\end{array}$} \\
\hline \multirow{2}{*}{$\begin{array}{c}\text { Apoyo } \\
\text { familiar }\end{array}$} & \multicolumn{2}{|c|}{ Localidad } \\
\cline { 2 - 3 } & Rural & Urbana \\
\hline No & 12 & 30 \\
\hline Si & 33 & 27 \\
\hline
\end{tabular}

La existencia de apoyo familiar también estaba significativamente asociada al estado civil $(\mathrm{p}-0,007)$, siendo las personas viudas quienes tenian menos posibilidades de acceso a la rehabilitación integral, seguido de las personas ca. sadas (ver tabla 3).

\begin{tabular}{|c|c|c|c|}
\hline \multicolumn{4}{|c|}{$\begin{array}{c}\text { Tabla } 3 \\
\text { Existeneia de apoyo familiar } \\
\text { seguin según estado civil ( } \mathbf{p}-0,007) .\end{array}$} \\
\hline \multirow{2}{*}{$\begin{array}{c}\text { Apoyo } \\
\text { familiar }\end{array}$} & \multicolumn{3}{|c|}{ Estado civil } \\
\cline { 2 - 4 } & Casado/a & Soltero/a & Viodo/a \\
\hline No & 15 & 17 & 10 \\
\hline Si & 41 & 1 & 18 \\
\hline
\end{tabular}

E. resto de factores analizados no presentaron una asociación significativa con el acceso a la rehabilitación integral. Respecto al sexo, fue un factor que no presentó en ningùn análisis una asociación significativa con el acceso a la rehabilitación integral $(\mathrm{p}-0,183)$. Los ingresos económicos tampoco fueron un factor asociado a la posibilidad de recibirla $(p \sim 0,60)$.

Resultados del análisis de regresión. Las únicas variables asociadas de forma independiente 
a un mayor acceso a la rehabilitación integral se muestran en la tabla 4.

\begin{tabular}{|c|c|c|}
\hline \multicolumn{3}{|c|}{$\begin{array}{c}\text { Tabla } 4 \\
\text { Variables explicativas del acceso } \\
\text { a la rehabilitación integral. }\end{array}$} \\
\hline Variable & $\begin{array}{c}\text { Coeficiente } \\
\text { (P-Valor) } \\
\text { [Variable } \\
\text { individual] }\end{array}$ & $\begin{array}{c}\text { Coeficiente/OR } \\
\text { (P-valor) } \\
\text { [ A justado al resio } \\
\text { de variables }]\end{array}$ \\
\hline Edad & $-0,17(-0,001)$ & $-0,226,0,8(<0,001)$ \\
\hline $\begin{array}{l}\text { Localidad } \\
\text { (urbana) }\end{array}$ & $1,37(0,001)$ & $2,85 / 17,4(40,001)$ \\
\hline $\begin{array}{c}\text { Apoyo } \\
\text { familiar } \\
\text { (si) }\end{array}$ & $1,1(0,011)$ & $-0,210,81(0,77)$ \\
\hline $\begin{array}{c}\text { Casado } \\
\text { (si) }\end{array}$ & $0,29(0,47)$ & $-0,35 / 1,42(0,64)$ \\
\hline
\end{tabular}

Se compararon las medias de edad entre am. bas localidades con un test $T$ de Student y no hubo diferencias significativas en la edad media de los pacientes de localidad rural y urbana $(\mathrm{p}-0,7)$, por lo que ambos factores eran independientes entre si. Tanto la edad como el tipo de localidad de residencia evidenciaron ser los factores claramente explicativos del acceso a la rehabilitación integral.

En los análisis se observó que dl resto de variables independientes actuaron como factores de confusión, quedando demostrado en los and. lisis iniciales $y$ finalmente comprobados en la regresión logistica multivariable.

\section{DISCUSIÓN}

En primer lugar, hay que destacar que la mitad de los pacientes que necesitan rehabilitación integral la reciben. La ausencia de centros públicos especificos en todas las localidades de residencia de los pacientes imposibilita el accesso de estos al tratamiento rehabilitador integral. Esta desigualdad implica que tengan que utilizar sus propios recursos personales para acceder a dicha rehabilitación.

Son dos los factores asociados claramente a la posibilidad de acceso a la rehabilitación integral. En primer lugar, la edad que, con una correlación perfecta, evidencia que a medida que aumenta la edad disminuye en la misma proporción la posibilidad de acceder a dicha rehabilitación. La frecuencia de padecer ictus aumenta progresivamente a partir de los 55 años ${ }^{*}$. Además, hay estudios que sugieren que una edad elevada impode el tratamiento rehabilitador pautado, al beneficiarse del mismo en mayor medida los jóvenes ${ }^{17}$, por lo que la edad constituye un factor determinante de la rehabilitación integral. El acceso a un tratamiento rehabilitador de acceso gratuito está limitado por la edad en ciertos centros. En los centros concertados, como el Centro Estatal de Atención Daño Cerebral (Ceadac), está limitado hasta los 55 años, y en Guttmann hasta los 60 años.

E. lugar de residencia (urbana o rural), es el segundo factor claramente asociado a la posibilidad de recibir una rehabilitación integral, en tanto que la accesibalidad a servicios sanitarios y sociosanitarios depende en buena parte de la proximidad $y$ facilidad de acceso a los mismos (distancia/transporte). Tanto en Castilla y León, territorio de residencia de los pacientes estudiados, como en otras comunidades autónomas de gran dispersión poblacional, los recursos asistenciales difieren mucho segün el territorio, no existiendo disponibilidad o acceso a estos en todas las zonas donde residen los pacientes de ictus. Residir en lugares mis pequeños, e incluso aislados por la falta de buenas comunicaciones, es un becho que dificulta el acceso a los recursos asistenciales y que condiciona mury claramente la posibilidad de acceder a una rehabilitaciỡn integral. Problemas deaccesibilidad a los recursos públicos y privados derivados del clima en el invierno, la falta de 
transporte püblico adaptado, la incomunicación, y el aislamiento, asi como la dificultad para encontrar gente que puch cuidar del paciente en el medio rural, son sin duda circunstancias que explican esta fuerte correlación. Otras son la dificultad de traslado de una zona rural a otra urbana, la inaccesibilisdad a tratamientos rehabilitadores en el lugar de residencia rural de la persona, la falta de transporte que se adapte a los borarios de tratamiento rehabilitador, la negativa de la persona a permanecer meses lejos de la localidad de origen.

El hecho de vivir sólo o bien con pareja/fa. miliares, al influir en la salud emocional y mental de las personas ${ }^{13}$, considerábamos que podria ser otro factor asociado a la rehabilitación integral. A prioni, no teniamos clara su posible influencia (positiva o negativa), ya que la soledad puede producir sentimientos negativos o implicar la ausencia de un familiar cuidador en el bogar, pero también puede hacer que la persona disponga de más tiempo para su rehabilitación al no tener cargas familiares. Ahora bien, atendiendo al resultado del análisis de regresión, la asociación que presenta este factor esta fuertemente condicionada por la edad, por lo que el estado civil no puede ser considerado como factor asociado, al menos en la muestra de nuestro estudio. La existencia de apoyo fa. miliar para acceder al tratamiento rehabilitador pensábamos que podria evidenciarse como un aspecto relevante. Las limitaciones, tanto fisicas como psiquicas, que pueden surgir ante la aparición de un ictus, hace que cobre todavia mayor importancia el papel de la familia en el acceso, mantenimiento y apoyo al tratamiento rehabilitador del paciente, formando asi parte activa de ese proceso. La familia es, habitualmente, el apoyo principal con que cuenta un paciente para afrontar con éxito los problemas a que da lugar la enfermedad, por lo que la existencia de apoyos y cuidados en el entorno familar parecia una circunstancia relevante que se asociaria positivamente al acceso a rehabilitación integral. Esta hipótesis, que inicialmente se confirmó en sentido inverso al esperado con el análisis de correlación, debe ser descartada, ya que el apoyo familiar está mucho mis presente en la zona rural que en la urbana. Esta circunstancia confirma que la variable "apoyo familiar" es un factor de confusión en el análisis de regresión. Abora bien, a pesar de que toda la literatura establece que contar con apoyo familiar es un elemento importante para asegurar una rehabilitación integral, el hecho de que aqui sea a la inversa se explica porque la mayoria de quienes cuentan con apoyo familiar viven en el medio rural (el valor chi-cuadrado entre apoyo familiar y localidad es significativo, $p-0,001)$, tal como se muestra en la tabla 1, siendo la localidad el factor condicionante de mayor peso entre ambos factores. Un estudio más amplio, donde el numero de pacientes con y sin apoyo familiar estuviera homogéneamente distribuido entre zona rural y urbana, sin duda permitiria confirmar o descartar de manera definitiva este factor.

De nuestra hipótesis inicial sobre factores asociados al accesso a la rehabilitación integral, resulta llamativo que los ingresos económicos y el sexo no presenten asociación estadisticamente significativa. El hecho de que el sexo no sea un factor significativo coincide con otros estudios que señalan que la capacidad funcional post-ictus al alta es similar en hombres y mujeres $^{1 \text { iต }}$. Respecto a los ingresos económicos, parecia obvio que, en un contexto de falta de acceso gratuito y universal a la rehabilitación integral ( $y$ considerando que bastantes de los servicios necesarios debian proporcionarse a nivel privado-mercantil, mediante pago), los ingresos mensuales del paciente podrian condicionar el acceso a los mismos. Sin embargo, esto no es asi en la muestra estudiada, debido a que la diferencia de ingresos medios entre quienes reciben rehabilitación y quienes no la 
reciben, es muy pequeña: en las personas que recibieron rehabilitación integral la media de ingresos mensuales es de 901,38 euros, y en la población que no recibió rehabilitación integral es de 856,04 euros.

Las limitaciones de este estudio han sido considerables, ya que en Castilla y León toda. via no está implantada en su totalidad la historia clinica electrónica del paciente, lo que ha supuesto tener que manejar varias herramientas informáticas de recopilación de datos. Las escalas aplicadas por la trabajadora social sanitaria de neurologia no estaban incluidas en la herramienta informática, lo que ha implicado tener que incorporarlas manualmente. Esto prolongó notablemente el proceso de toma de datos. Otra limitación importante fue la dificultad de acceso a datos económicos precisos de los pacientes que estaban ingresados en el hospital, la buisqueda activa de familiares (algunos fuera de la localidad, algunos sin relación con el paciente) para conseguir datos, y la gran dificultad en el caso de pacientes de los que al ingreso se temia por su vida. Hubo que realizar un trabajo de investigación y recopilación de datos en los casos en los que el paciente no podia expresarse. En otros casos, se contó con la ayuda de un logopeda y con un tablero de comprensión para poder realizar una entrevista.

La falta de garantia pública de acceso a la rehabilitación integral con carácter gratuito y universal en nuestro pais se evidencia en el hecho de que no existe ninguin centro de rehabilitaciốn integral (público o privado) en la mayoria de las comunidades autónomas. Esta situación hace que la "gestión de caso social" desarrollada por el trabajador sanitario de neurologia resulte determinante para implementar dicha rehabilitación integral, al movilizar diversos y multiples recursos sociales, naturales, institu= cionales y privados, que permiten la rehabalitación integral del paciente susceptible de recibirla en territorios sin los recursos públicos adecuados. Asi contribuyen a la recuperación del paciente y le garantiran una vida lo más autónoma posible.

\section{AGRADECIMIENTOS}

Agradecemos profundamente a Ricardo E. Ander-Egg Aguilar (Universidad de Barcelona) el detallado y minucioso trabajo de análisis de datos que ha realirado $y$ que nos ha permitido obtener los resultados de investigación que aparecen en este articulo.

\section{BIBLIOGRAFIA}

1. Organización Mundial de la Salud. Las 10 priacipales causas de defuncsine. [Internet] 2018. [acrese of de marwo de 2019]; Dikporible en: hops: Fwew who int/news-rocen'

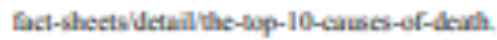

2. Noé-Sebustile E, Balasch-Bernie M, Colomet-Foet C, Molinet-Muhoo B, Rodriguez Slachez-Leiva C, Ugart Pes al. Jetus y discapacidad: esloudio loagitadinal en pocieates con discapacialad moderade-grave tras un icous incluibos en un programa de relabsilitacica multidisciplitat. Rev Neurd. 2017; 64(9),385-392. Boi. 10.335ka m.6409.2016527.

3. Olmado Voga V, Arrane Banderas M. Trabajo Social Sanitario: Intervencicie con pacientes oce icous detde el Servicio Madios at Neurologia del Hospital Clinioe Universidario de Valladolid. Trab Soe y salud. $2017 x \mathrm{k8}) \times 39-43$.

4. Arias Cuadado A. Rehabilitación det ACV. evaluación,

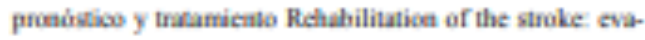
luatice, progrosis and trewanene. Calicia Clin [Intemet] 2009 [acteso 18 de julio de 2019] 70(3) L-40. Dieporible cn: ltrps:/galiciacliaica infoPDF/S/81.par.

5. Moyano A. E accidente ceretorov4ascular desde la mirala del rehabilitadoe. Rer Hosp Clin Univ Chile 2010,21:354. Disponàle en anpollwww.redclinica.cV Portak0Users 014/14/14, Publicaciones/Revistabeidente_cerebovasculy_desde_mirala_tchabiliodie par. 
6. Duarte E, Alcesso B, Fernindez. MU, Fernindez.JM, Flúrez M, Gascia-Mceles I et al. Rehabilitación del ictus: modebo asibseacial. Reoconendacsiones de la Sociodad Eqpâtola Ar Rehabilitacice y Modicina Fisica, 2009. Rehubilitacsin (Madr). 2010,44(1)60-3. Doi: 10.1016j, th 2009.10 .001$.

7. Millin Mulaz R. Modelos de schabilitacsion ideal. [Mosografa en laternet]. Cali (Coloenbia) Fundacsin Ideal Julio H. Cakajk, 2012 [acteso 17 de actuhe de 2019) Recuperads de: Imps:/wew fundacicaideal arg col shesUefault files/publicacioneslarchivos/modelo-felabiliacice-integral-ideul2012 pdf

8. Laxe S. El icous en las mujeres. Sobre rucdas. [Internet] 2018 [A cceso 25 de marm de 2019] (x93) 22-25. Disponible

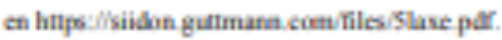

9. Simb-Nograera C, Herníndez-Mbalate A, MubosRedziguez D es al. El efecto del estado cival y de la coenvivencia en parcja en la salua. Rev Espotola lmestig Saciolbraicas. 2015,151:141-65.

10. Moltó JM, Lecanos A, Cactellivi E, Villanueva V. (2007). Las Agudas. Recursas Sociales tras un Icoss. En Castellvi es al.Gufu de informacióa al paciente coe ictus (pp.103-107). VuleaciacGeacralitat Valenciana.

11. Hunser D. Matplotlib: A 2D Graphies Envirenent. Comput Sci Eng. 2007; 9095-9. Doi. 10.1100 MCSE 2007.55.

12. Mckinney W. Duca Structures for Statistical Computing in Python. Procetlings of the 9h Python in Science Conference. 2010,51-56.

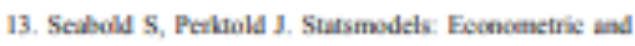
seristical modeling with pythoe. Procetings of the 9th
Pydhon in Sciencr Coefierence. [Intemet]. 2010, [uccews 18 de julio de 2019]; 57-61. Disponible en: linps:/paff scman-

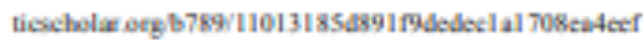
97a62.par? ga=2. 1648205402079434522.1564390394 . 968556119.1564390394

14. Podregosa F, Varoquaus G, Geanfort A, Michel V, Thirice B, Grisel O, Blcedel M, Pretseahofer P, Wetiss R es al. J Mach Leam Res. [Internet]. 2011 , [laternet] [acceso 18 de julio de 2019]; 12: 2825-2830. Dispoeaible en. hnp./eww.jmlz org/papers/volume12/pedregovalla' pedtegowal la par.

15. The jenoni pooject. Vervion 1.0 [Compuber Softuare] 2019 [cibudo 18 de julio de 2019]. Dispoaible en: hnpo: www.jameviorg.

16. Fernandex-Gímex FJ, Hemindex F, Argandiela L. et al. Fannacologia de la neuroprolectión en el ictus bucénico aguda. Rev Neurol. 2006,47:253-60.

17. Sincher M. Las accesaluder sociales oeiginadas a consecuencia de la enfermedad en pacienses adulas hospinalirulos [oesib doctoral]. Madral: Universabad Complutense de Madrid, 2016.

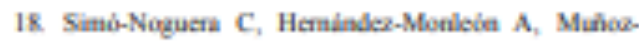
Rodrigues D et al. El efocto del evado civil y de la convivencia en pareja en la salud Rev Espabola lavertig Sociobigion. 2015,151:141-65.

19. Petulver Ptrez F, Lèpez Barrio MJ, Marín López T, Sarin Marin D. El icfus en las majeres. perspectina de getne-

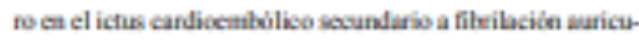
lax. Revisa ENE de Enfermeria. [Inemes]. 2015. [htemet]. [aceese 18 \& julio de 2019] 9/2). Dikponîle en Inpileneenfenneria oeg/ujwindexplap/ENE/articleview.497. 
Autores: Verónica Olmedo Vega, María-José Aguilar-Idáñez y Juan Francisco Arenillas Lara

Fecha de envío: 14/01/2021

Fecha aceptación: 10/05/2021

Fecha prevista publicación: Previsto que sea publicado en el número 73_01 correspondiente a la primera quincena de julio.

\section{Notificación de aceptación}

\section{RE: 2021037_Olmedo Vega_Aceptación_Revista de Neurología}

De: Secretaria Viguera 〈secretaria@ @iguera.com>

Enviado: lunes, 10 de mayo de 2021 13:13

Para: Olmedo Vega, Verónica 〈volmedo@saludcastillayleon.es〉

Asunto: 2021037_Olmedo Vega_Aceptación_Revista de Neurología

Distinguido/a Prof/a. Olmedo Vega,

- Celebramos comunicarle que su artículo "Rehabilitación integral post-ictus: efectos a largo plazo y factores socioambientales condicionantes del acceso" con referencia 2021037, ha sido aceptado para su publicación tras valoración por el comité científico.

Agradecemos su confianza en Revista de Neurología para la edición de su artículo, y esperamos poder seguir colaborando con usted en la difusión de la comunicación cientifica en español.

Muy atentamente,

Secretaría Editorial [secretaria@viguera.com]

Viguera Editores S.L.U.

Plaza Tetuán $72^{a}$ Planta

08010 Barcelona

Tel: 932478188

Fax: 932317250

whw.viguera.com

El presente documento y sus anexos contienen datos de carácter personal, por lo que se le advierte de su obligación de conocer y cumplir lo establecido en la Ley Orgánica 15/1999 de 13 de diciembre, de protección de datos de carácter personal, Real Decreto 994/1999 de 11 de junio, por el que se aprueba el Reglamento de medidas de seguridad de ficheros automatizados que contengan datos de carácter personal, y demás disposiciones vigentes. En cualquier momento usted podrá ejercitar los derechos de acceso, rectificación, oposición y, en su caso, cancelación, comunicándolo por escrito con indicación de sus datos a Viguera Editores S.L.U., con domicilio en la Plaza Tetuán, $72^{\circ}$, Barcelona (España).

... AVISO SOBRE CONFIDENCIALIDAD. La legislación española ampara el secreto de las comunicaciones. Este correo electrónico y sus anexos son estrictamente confidenciales. Si Vd. no es el destinatario del mismo, por favor, notifíquenoslo inmediatamente y destruya el original. No deberá copiar este mensaje ni sus anexos o usarlo para propósito alguno, ni divulgar su

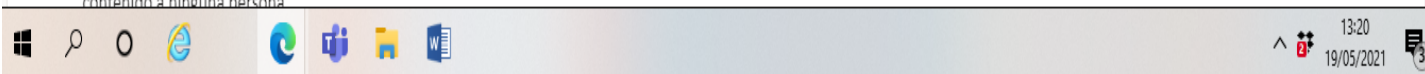




\title{
RESUMEN
}

Introducción: La recuperación de todas las funciones cerebrales afectadas tras un ictus es esencial para la calidad de vida del paciente, resultando clave la rehabilitación integral. Objetivos: 1) Identificar factores sociales y ambientales condicionantes del acceso a rehabilitación integral post-ictus. 2) Valorar efectos a largo plazo de la rehabilitación integral en la funcionalidad del paciente.

Pacientes y Método: Se estudian 171 pacientes consecutivos ( 84 mujeres y 87 hombres) hospitalizados en 2015 en Servicio de Neurología con primer ictus isquémico, sin dependencia funcional previa, candidatos a rehabilitación integral. Se analizan diversas variables socioambientales y clínicas potencialmente asociadas al acceso a la misma. Se estudia el impacto pronóstico a largo plazo (período medio de 54 meses) sobre la situación funcional mediante índice Barthel.

Resultados: La edad media de los pacientes es de 69 años. Sólo el 53\% pudo acceder a la rehabilitación integral recomendada. Resultaron variables predictoras del acceso: residencia en medio urbano (OR: 2,957, (IC 95\% 1,067-8,199), $\mathrm{p}=0,037$ ), complemento con RHB privada (OR: 2,890 , IC 95\% 1,130-7,392), $\mathrm{p}=0,027$ ), mejor Rankin al alta (OR: 22,437, (IC 95\% 3,247-155,058), $\mathrm{p}=0,014$ ). Tras seguimiento medio durante 54 meses, de los 137 supervivientes, el acceso a rehabilitación integral post-ictus se asoció independientemente a mejor situación funcional a largo plazo (OR: 12,441, (IC 95\% 4,7$32,5), \mathrm{p}<0,001)$.

Conclusiones: La rehabilitación integral post-ictus está asociada a un mejor pronóstico a largo plazo, pero el acceso a la misma está condicionado por factores ambientales y sociales tales como el lugar de residencia y la posibilidad de contratar servicios privados. Palabras clave: Accesibilidad a los servicios de salud. Calidad en la atención en salud, Acceso y evaluación. Factores determinantes de salud. Rehabilitación ictus. Ictus isquémico. Trabajo social.

\begin{abstract}
Introduction: Recovery of all brain functions affected after stroke is essential for the patient's quality of life, with comprehensive rehabilitation key.

Objectives: 1) Identify social and environmental factors affecting access to comprehensive post-ictus rehabilitation. 2) Assess long-term effects of comprehensive rehabilitation on patient functionality.

Patients and Method: 171 consecutive patients ( 84 women and 87 men) hospitalized in 2015 in Neurology Service with first ischemic stroke, without prior functional dependence, candidates for comprehensive rehabilitation are studied. Various socioenvironmental and clinical variables potentially associated with access to it are analyzed. The long-term prognostic impact (average period of 54 months) on the functional situation is studied using the Barthel index.

Results: The average age of patients is 69 years. Only 53\% were able to access the recommended comprehensive rehabilitation. Predictor variables of access were resulted: residence in urban environment (OR: 2,957, (95\% CI 1,067-8,199), p-0.037), complement with private RHB (OR: 2,890, 95\% CI 1,130-7,392), p-0.027), best Rankin to high (OR: 22,437, (95\% CI 3,247-155,058), p-0.014). After average follow-up for 54 months, of the 137 survivors, access to comprehensive post-ictus rehabilitation was independently associated with better long-term functional situation (OR: 12,441, (95\% CI 4.7-32.5), $\mathrm{p}<0.001)$.
\end{abstract}


Conclusions: Comprehensive post-ictus rehabilitation is associated with better long-term prognosis, but access to it is conditioned by environmental and social factors such as the place of residence and the possibility of contracting private services.

Keywords: Accessibility to health services. Quality in health care, access and evaluation. Determinants of health. Stroke rehabilitation. Ischemic stroke. Social work.

\section{INTRODUCCIÓN}

Se estima que 1 de cada 4 personas sufrirá un ictus a lo largo de su vida [1], siendo la primera causa de discapacidad en personas adultas, con predominio del grupo de edad de 60 a 69 años [2]. Discapacidad ocasionada por secuelas derivadas del déficit funcional de las áreas cerebrales lesionadas por el ictus, que son diversas y afectan a la funcionalidad de la persona a todos los niveles [3, 4].

La American Heart Association-Stroke Outcome Classification establece 6 áreas funcionales afectadas por déficits neurológicos post-ictus: "cognitiva, emocional, lenguaje-comunicación, motora, sensitiva y visual" [5]. Los efectos inter-retro actúan entre sí, generando déficits complejos que van más allá de cada área de función [6].

La rehabilitación (RHB) post-ictus persigue la recuperación de las funciones cerebrales perdidas por el paciente. El plan estratégico nacional para el tratamiento integral de las enfermedades neurológicas de la Sociedad Española de Neurología 2012, señala que el cuidado de los pacientes con ictus debe ser integral [7]. Es decir, que cubra todo el espectro de déficits neurológicos ocasionados por el ictus, y que no se centre exclusivamente en el déficit motor.

Debe mejorarse el acceso al tratamiento rehabilitador [8], identificando factores sociales y ambientales que influyen en el acceso al mismo, dado que, en la práctica, la RHB integral no está garantizada universal ni gratuitamente. El Trabajo Social de neurología, integrante de la unidad de ictus, incide en el acceso, activando diversos apoyos del entorno social del paciente, movilizando recursos externos y facilitando la utilización de aquellos que permiten la RHB integral. Los criterios de rehabilitación integral los establece el equipo responsable del proceso asistencial, integrado por diferentes profesionales competentes en su área de intervención, aunque muchos profesionales intervengan a lo largo de todo el proceso [9].

En este contexto, diseñamos el estudio con dos objetivos: 1) identificar factores sociales y ambientales asociados al acceso a la RHB integral recomendada por el equipo multidisciplinar de ictus, y 2) evaluar el efecto a largo plazo de la RHB integral sobre la autonomía funcional de los pacientes que acceden a la misma.

\section{PACIENTES Y MÉTODOS}

Diseño: Estudio unicéntrico, observacional, retrospectivo, de seguimiento a largo plazo de pacientes consecutivos con un primer episodio de infarto cerebral agudo, ingresados en un Centro Terciario de Ictus durante el año 2015, considerados candidatos a recibir RHB integral por el equipo multidisciplinar de la unidad de ictus.

Sujetos de estudio (criterios de inclusión): primer ictus isquémico, mayores de 18 años, infarto cerebral con afectación de al menos dos sistemas funcionales y una puntuación al alta en la escala modificada de Rankin $\geq 1$, capacidad cognitiva para seguir tratamiento rehabilitador (sin presencia de deterioro cognitivo significativo), motivación para recibirlo, perteneciente al Área de Salud de referencia del Hospital. Criterios de 
exclusión: presentar alguna condición que requiera una intervención inmediata (p. ej., ideación suicida), capacidad cognitiva que impida comunicarse adecuadamente, estar o precisar tratamiento paliativo.

El Comité Ético de Investigación Clínica aprobó el protocolo de investigación. La participación en la investigación no supuso para los pacientes ningún incentivo económico o de otro tipo. Todos los participantes otorgaron consentimiento para poder incorporar la información de la historia clínica y social de forma encriptada en la base de datos del estudio, cumpliendo la Ley de Protección de Datos de Carácter Personal.

\section{Protocolo de inclusión y seguimiento multidisciplinar de los pacientes}

Los pacientes fueron revisados clínica y socialmente desde y durante su ingreso hospitalario por el equipo multidisciplinar. Se elaboró una base de datos Excel, donde se recogen durante la hospitalización los pacientes que cumplen criterios para RHB integral: primer ictus, edad, sexo, diagnóstico ingreso, situación funcional mediante escala de Rankin modificada [10], escala de riesgo social al ingreso, gravedad al ingreso según escala de ictus del National Institute of Health (NIHSS) [11], familia, localidad de residencia (volante de empadronamiento), unidad de convivencia (certificado de convivencia), situación laboral e ingresos económicos (nómina, certificado de pensiones o prestaciones).

Al ingreso hospitalario de pacientes por primer ictus isquémico se emite interconsulta a Trabajo Social de neurología y Rehabilitación, que valoran inicialmente al paciente durante las primeras 24-48 horas. Previamente al alta se convoca reunión del equipo multidisciplinar compuesto por: neurólogo/a de la unidad de ictus, trabajadora social de neurología, neurólogos/as de planta, médica rehabilitadora, fisioterapeuta, logopeda, supervisora de enfermería de neurología y celador. En dicha reunión se elabora un plan individualizado de rehabilitación integral que cubra todas las áreas funcionales afectadas. Posteriormente, Trabajo Social visita al paciente para verificar su consentimiento del tratamiento rehabilitador, comprobando nivel de conciencia y capacidad cognitiva para adherencia al mismo. Al alta hospitalaria, en su historia clínica se recogen: informe clínico de alta, informe social, puntuación en Barthel y tratamiento rehabilitador a recibir. Antes de la finalización del tratamiento rehabilitador se contacta con paciente, familia y equipo médico-social y se confirma si el paciente ha podido acceder a la RHB integral pautada, recogiendo como variable la necesidad de utilizar servicios/recursos privados (no gratuitos). En los 4-6 meses posteriores al inicio de RHB integral se valora al paciente por Neurología y Trabajo Social, contando con un seguimiento a largo plazo en Trabajo Social mediante entrevistas y valoraciones cada 6-9 meses. La última visita de evaluación final (realizada en los 47 a 55 meses posteriores al alta) consiste en entrevista presencial con paciente y familia administrando de nuevo el índice Barthel. El protocolo seguido se muestra en la Figura.

\section{Definición de las variables basales}

Se registró información correspondiente a las variables basales mencionadas anteriormente en el protocolo. El número de convivientes en el domicilio se codificó comprobando si el paciente vivía solo, o si vivían 2, 3 o más personas. El lugar de residencia, rural o urbano se determinó por el número de habitantes del municipio (de acuerdo con la clasificación del Instituto Nacional de Estadística, INE), siendo urbano el municipio de Anonimizado y rural el resto de los municipios de la provincia. La accesibilidad a la vivienda se codificó verificando si es adecuada (adaptada para permitir 
la deambulación en el domicilio) o no adecuada (no permite la permanencia de la persona en la misma, existencia de escaleras, bañera y otras barreras físicas). El riesgo social al ingreso se determinó mediante aplicación de la Escala Gijón. Se evaluó la necesidad de complementar la RHB con recursos privados (modalidad privada sí/no) pagados por el propio paciente o familia.

\section{Estimación del pronóstico funcional a largo plazo}

Para la valoración de la evolución funcional del paciente se empleó índice Barthel, que mide la capacidad para realizar 10 actividades de la vida diaria, consideradas básicas, permitiendo establecer grado de independencia funcional. Esta escala se aplicó en dos momentos: al alta hospitalaria del paciente (en 2015) y entre el 1/02 y el 10/03 de 2020, considerándose largo plazo un período igual o superior a los 4 años desde el alta. Se codificó según estudio [12] publicado en la revista de la Sociedad Española de Neurología, agrupándose por categorías de dependencia: dependencia total (puntuación 0-20); dependencia grave (21-40); dependencia moderada (41-60); dependencia leve (6190) e independencia (91-100).

\section{Análisis estadístico}

Las variables se describen utilizando media y desviación estándar o mediana y su rango intercuartílico, en función de su mejor adecuación a las características de la variable. Las cualitativas se presentan según su distribución de frecuencias. Mediante test Chicuadrado de Pearson se analizó asociación de variables cualitativas. En el caso de que el número de celdas con valores esperados menores de 5 es mayor de un $20 \%$, se ha utilizado el test exacto de Fisher o el test Razón de verosimilitud para variables con más de dos categorías. Las comparaciones de los valores cuantitativos se han realizado mediante la prueba $\mathrm{T}$ de Student para muestras independientes o la prueba U de Mann Whitney.

Se ha realizado análisis univariante de regresión logística para cada una de las variables del estudio asociadas a la rehabilitación integral y pronóstico funcional favorable definido por $\mathrm{BI}>60$. Aquellas que han resultado ser estadísticamente significativas a nivel 0,1 han sido introducidas en un modelo de regresión logística multivariable.

Se utilizó el programa estadístico IBM SPSS Statistics versión 24.0 para Windows. Son considerados estadísticamente significativos valores de $\mathrm{p}<0,05$.

\section{RESULTADOS}

\section{Características basales de la muestra}

Se incluyó a los 171 pacientes que cumplían criterios para una rehabilitación integral; la edad media fue de 69,73 (desviación estándar [DE]:16,38). La edad media de los que sí accedieron a rehabilitación integral es de 65,23. La Tabla I muestra las características basales de los pacientes. La mayor parte conviven con una sola persona, no están jubilados y tienen ingresos medios de 1.170,7 euros mensuales. El 77,2\% tiene su vivienda en propiedad y el 55,6\% presentaron riesgo social al alta. En cuanto al tratamiento rehabilitador, destaca que el $24 \%$ recibe únicamente rehabilitación pública.

Tabla I. Características basales ( $\operatorname{casos} \mathbf{n}=171)$ 


\begin{tabular}{lll}
\hline Sexo & Mujer: $84(49,1 \%)$ & Hombre: $87(50,9 \%)$ \\
Edad & Media: 69,73 & Desviación: 16,38 \\
Convivientes & Solo: $60(35,15 \%)$ & 2 personas: $87(50,9 \%) \quad \geq 3$ pers.: $24(14 \%)$ \\
Situación laboral & Jubilado: $62(36,3 \%)$ & No Jubilado: $109(63,7 \%)$ \\
Economía familiar & Media: 1170,7 euros/mes & Desviación: 389,91 \\
Lugar de residencia & Rural: $50(29,2 \%)$ & Urbana: $121(70,8 \%)$ \\
Accesibilidad vivienda & Adecuada: $87(50,9 \%)$ & No adecuada: $84(49,1 \%)$ \\
Propiedad vivienda & Si: $132(77,2 \%)$ & No: $39(22,8 \%)$ \\
Riesgo social ingreso & Si: $95(55,6 \%)$ & No: $76(44,4 \%)$ \\
Ranking alta hospital & $0+2: 100(58,5 \%)$ & $3: 24(14 \%)$ \\
NIHSS alta media & Media: 6,78 & Rango: $24(15,2 \%)$ \\
Rehabilitación privada & Si: $130(76 \%)$ & No: $41(24 \%)$ \\
Hipertensión arterial & Si: $92(53,8 \%)$ & No: $79(46,2 \%)$ \\
Diabetes & Si: $41(24 \%)$ & No: $130(76 \%)$ \\
\hline
\end{tabular}

\section{Variables asociadas al acceso a la rehabilitación integral}

La relación entre las variables basales y el acceso a una rehabilitación integral tras el alta hospitalaria aparecen en la Tabla II. Las variables asociadas en el modelo univariante fueron:

Tabla II. Relación entre variables basales y acceso rehabilitación integral (n=171)

\begin{tabular}{|c|c|c|c|}
\hline Variables & $\begin{array}{l}\text { RHB Integral: } \\
\text { Si }\end{array}$ & RHB Integral: No & P-valor \\
\hline MUESTRA & 91 personas & 80 personas & \\
\hline \multicolumn{4}{|l|}{ SEXO } \\
\hline Hombre & $52(57,1 \%)$ & $35(43,8 \%)$ & 0,080 \\
\hline Mujer & $39(42,95 \%)$ & $45(56,3 \%)$ & \\
\hline \multicolumn{4}{|l|}{ EDAD } \\
\hline$\geq 81$ años & $21(23,1 \%)$ & $30(37,5 \%)$ & 0,001 \\
\hline 61-80 años & $39(42,9 \%)$ & $41(51,3 \%)$ & \\
\hline$\leq 60$ años & $31(34,1 \%)$ & $9(11,3 \%)$ & \\
\hline \multicolumn{4}{|l|}{ CONVIVIENTES } \\
\hline Solo & $36(39,6 \%)$ & $24(30 \%)$ & 0,026 \\
\hline 2 personas & $38(41,8 \%)$ & $49(61,3 \%)$ & \\
\hline 3 o más personas & $17(18,7 \%)$ & $7(8,8 \%)$ & \\
\hline \multicolumn{4}{|l|}{ ACTIVIDAD } \\
\hline \multicolumn{4}{|l|}{ LABORAL } \\
\hline Jubilado & $34(37,4 \%)$ & $28(35 \%)$ & 0,748 \\
\hline No jubilado & $57(62,6 \%)$ & $52(65 \%)$ & \\
\hline ECONOMIA (MEDIA) & $1197,7422 €$ & $1139,9375 €$ & 0,335 \\
\hline \multicolumn{4}{|l|}{ RESIDENCIA } \\
\hline Rural & $30(37,5 \%)$ & $20(22 \%)$ & 0,026 \\
\hline Urbana & $50(62,5 \%)$ & $71(78 \%)$ & \\
\hline \multicolumn{4}{|l|}{ ACCESIBILIDAD } \\
\hline \multicolumn{4}{|l|}{ VIVIENDA } \\
\hline Adecuada & $56(61,5 \%$ & $31(38,8 \%)$ & 0,003 \\
\hline No adecuada & $35(38,5 \%)$ & $49(61,35 \%)$ & \\
\hline \multicolumn{4}{|l|}{ PROPIEDAD } \\
\hline \multicolumn{4}{|l|}{ VIVIENDA } \\
\hline $\mathrm{Si}$ & $63(69,2 \%)$ & $69(86,3 \%)$ & 0,008 \\
\hline No & $28(30,8 \%)$ & $11(13,8 \%)$ & \\
\hline \multicolumn{4}{|l|}{$\begin{array}{l}\text { RIESGO SOCIAL } \\
\text { INGRESO }\end{array}$} \\
\hline $\mathrm{Si}$ & $50(54,9 \%)$ & $45(56,3 \%)$ & 0,864 \\
\hline No & $41(45,1 \%)$ & $35(43,8 \%)$ & \\
\hline
\end{tabular}




\begin{tabular}{llll}
\hline RANKIN ALTA $^{\mathbf{a}}$ & $60(65,9 \%)$ & $40(50 \%)$ & 0,019 \\
$\leq 2$ & $14(15,4 \%)$ & $10(12,5 \%)$ & \\
3 & $12(13,2 \%)$ & $14(17,5 \%)$ & \\
4 & $5(5,5 \%)$ & $16(20 \%)$ & 0,442 \\
5 & 7,08 & 6,51 & \\
NIHSS ALTA MEDIA & & \\
MODALIDAD & & & 0,010 \\
PRIVADA & $62(68,1 \%)$ & $68(85 \%)$ & \\
$\mathrm{Si}$ & $29(31,9 \%)$ & $12(15 \%)$ & 0,037 \\
No & & & \\
DIABETES & $16(17,6 \%)$ & $25(31,3 \%)$ & \\
$\mathrm{Si}$ & $75(82,4 \%)$ & $55(68,8 \%)$ & 0,127 \\
No & & & 0,002 \\
HIPERTENSIÓN & & $48(60 \%)$ & \\
ARTERIAL & $44(48,4 \%)$ & $32(40 \%)$ & \\
Si & $47(51,6 \%)$ & $14,68 \mathrm{kms}$ & \\
No & $85,023 \mathrm{kms}$ & & \\
DISTANCIA (MEDIA) & & & \\
\hline
\end{tabular}

${ }^{a}$ mRS: Escala de capacidad funcional de Rankin Modificada (mRS $\geq 2$ : discapacidad moderada, moderadamente grave o grave); ${ }^{\mathbf{b}}$ NIHSS: Escala de gravedad del Ictus del National Institute of Health.

Cuando estas variables fueron llevadas a un modelo de regresión logística multivariable, resultaron variables asociadas al acceso a RHB integral las mostradas en Tabla III.

Tabla III. Modelo de regresión logística multivariable para factores predictores de acceso a rehabilitación integral

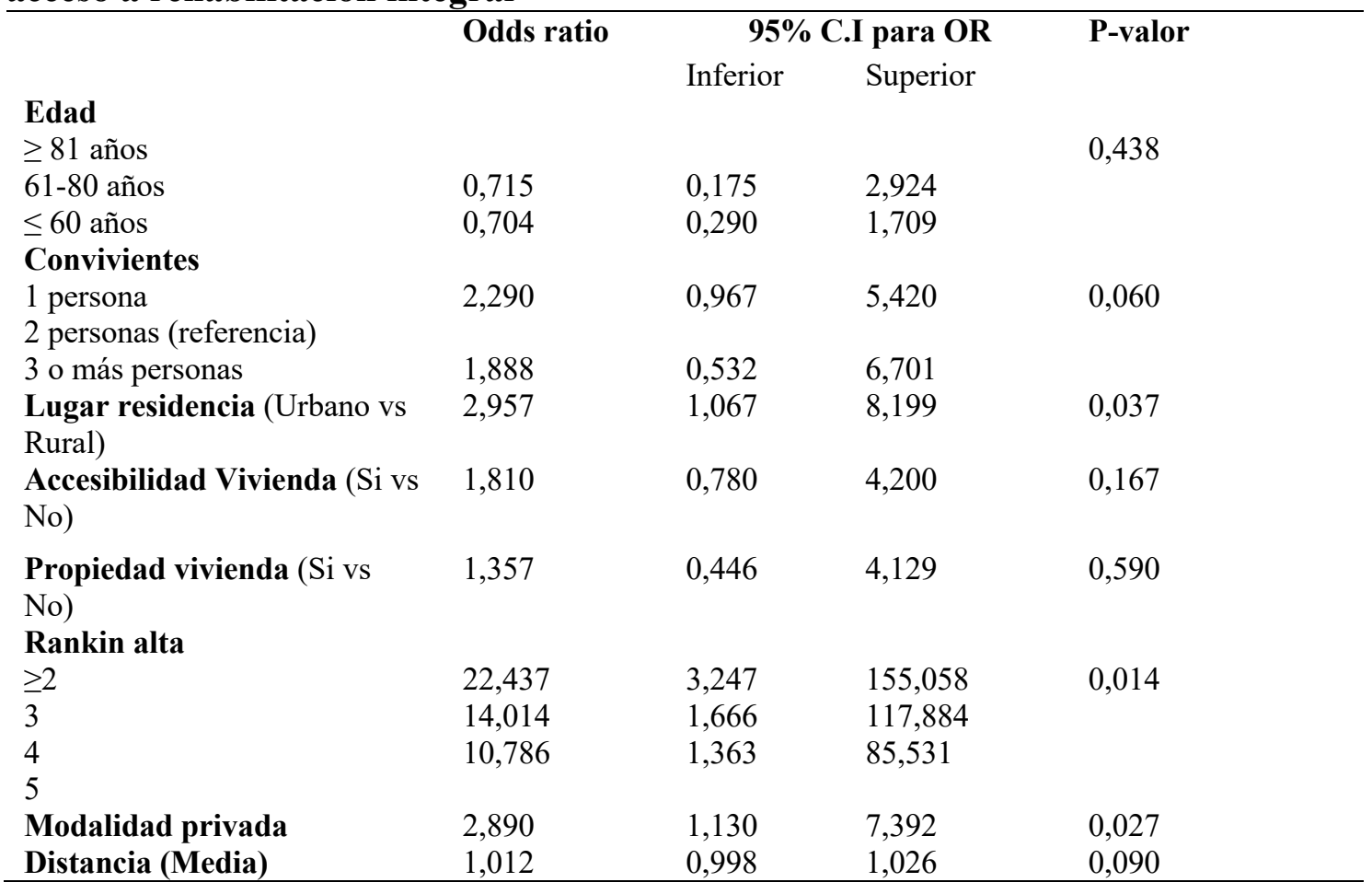

El lugar de residencia, urbano vs rural; el Rankin al alta y la modalidad privada fueron las variables que evidenciaron una relación significativa con el acceso a RHB integral.

\section{Impacto de la rehabilitación integral sobre el pronóstico funcional a largo plazo}

Se analizó el efecto a largo plazo de la RHB integral en la autonomía funcional de pacientes que fueron alta hospitalaria en el año 2015 y sobrevivían en marzo de 2020. La 
comprobación de supervivencia de los 171 pacientes de la muestra inicial se realizó a través de su historia clínica y el registro civil, habiendo fallecido 34 a fecha 10/03/2020. Llegaron al final del estudio 137 pacientes, cuya mediana de puntuación en el BI fue de 56,3 en 76 pacientes $(55,5 \%)$ alcanzando el rango de dependencia leve $(\mathrm{BI}>60)$.

De los pacientes que fueron alta en el año 2015,77 pacientes $(56,2 \%)$ presentaban dependencia grave (21-40) o dependencia moderada (41-60) y 60 pacientes $(43,8 \%)$ dependencia total (0-20). En marzo de 2020, los pacientes con dependencia total eran 26 (19\%), con dependencia grave y moderada 35 pacientes $(35,5 \%)$, y con dependencia leve $76(55,5 \%)$. Transcurrido un período medio de 54 meses desde el inicio del tratamiento rehabilitador integral (período entre 50 y 62 meses, dependiendo de mes de alta hospitalaria durante 2015), se constata una reducción del número de pacientes con dependencia total, grave y moderada respecto a su situación al alta hospitalaria, estadísticamente significativa $(\mathrm{p}=0,004 \mathrm{y} \mathrm{p}=0,001)$. Es decir, los pacientes con $\mathrm{BI}>60$ puntos a largo plazo aumentan al $55,5 \%$ de la muestra frente al $100 \%$ de pacientes con $\mathrm{BI}<60$ puntos al alta hospitalaria.

Esta asociación entre RHB integral y mejor evolución funcional se ilustra en el Gráfico, que muestra cómo el grupo que recibió RHB integral presentó un mayor incremento de la puntuación en Barthel durante el seguimiento.

\section{Gráfico: Evolución temporal del pronóstico funcional y su relación con la RHB integral}

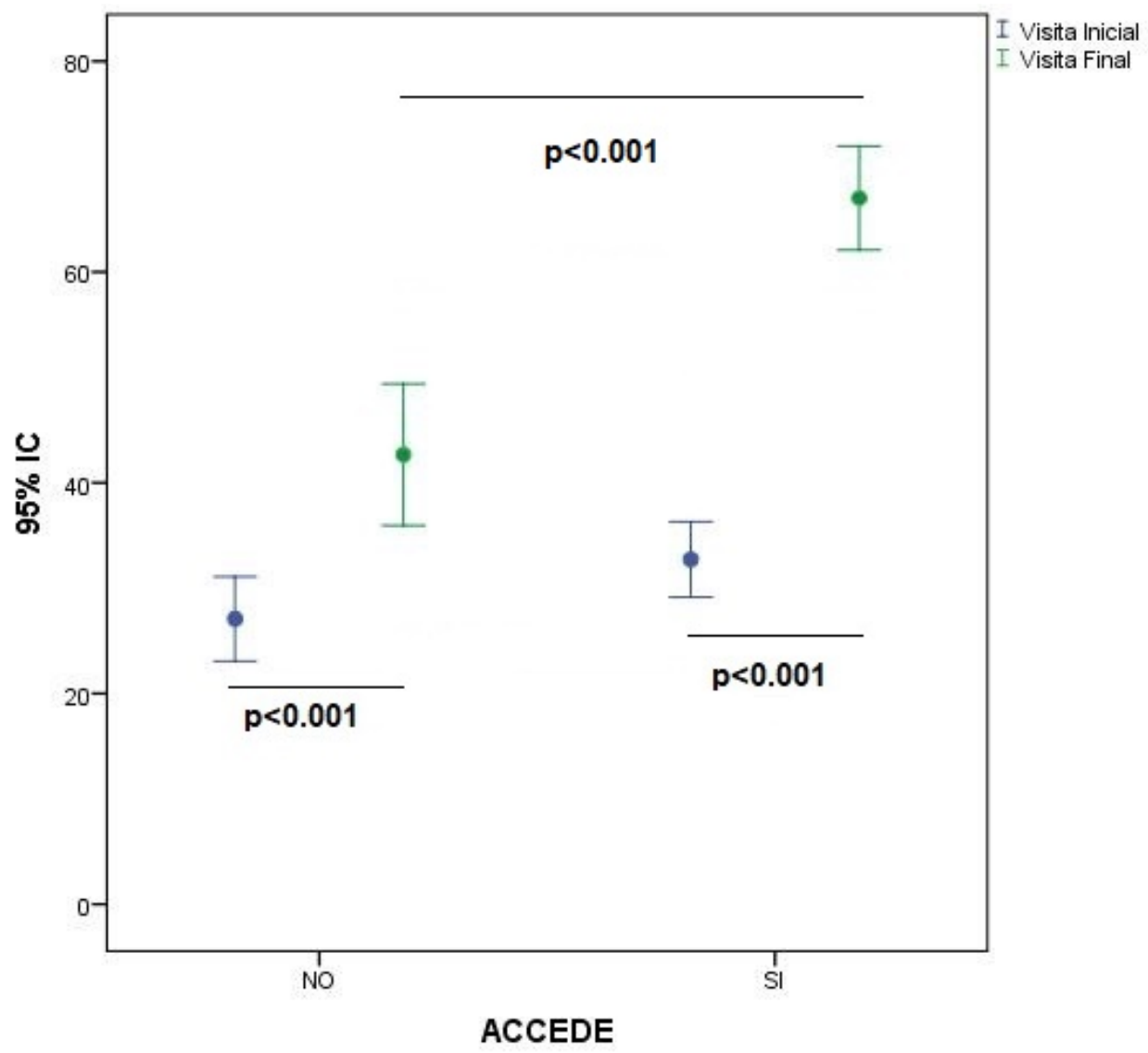

La Tabla IV muestra el resultado del análisis de regresión logística no ajustado entre cada una de las variables basales y el pronóstico funcional a largo plazo. Cuando estas variables fueron llevadas a un modelo de regresión logística multivariante, resultaron como 
predictores de mejor pronóstico funcional las siguientes: acceso a rehabilitación integral (OR: 12,441, (IC 95\% 4,7-32,5), p<0,001), Rankin al alta (OR: 6,644, (IC 95\% 1,2-36,6), $\mathrm{p}=0,030)$ y la menor distancia en kilómetros al centro de rehabilitación (OR: 0,997, (IC $95 \% 0,994-0,999), \mathrm{p}=0,024)$.

Tabla IV. Variables basales asociadas con una mejor evolución funcional a largo plazo

\begin{tabular}{|c|c|c|c|c|c|}
\hline & \multirow[t]{2}{*}{$\operatorname{Exp}(B)$} & \multicolumn{2}{|c|}{ 95\% C.I. para Exp (B) } & \multirow[t]{2}{*}{ Sig. } \\
\hline & & & Inferior & Superior & \\
\hline \multicolumn{2}{|c|}{ RHB INTEGRAL (Si vs No) } & 7,123 & 3,341 & 15,187 & $<0,001$ \\
\hline \multirow{3}{*}{ Edad } & $\geq 81$ años & 1,555 & ,584 & 4,135 & ,377 \\
\hline & $61-80$ años & 1,165 &, 530 & 2,558 &, 704 \\
\hline & $\leq 60$ años & & & & 674 \\
\hline \multirow[t]{3}{*}{ Convivientes } & $\geq 3$ & & & & ,778 \\
\hline & 2 personas & 1,276 & ,496 & 3,288 & 613 \\
\hline & 1 persona & 1,276 & 601 & 2,709 &, 525 \\
\hline \multirow[t]{4}{*}{ Rankin alta } & $\leq 2$ & 2,139 &, 589 & 7,771 & ,248 \\
\hline & 3 & 1,750 & 348 & 8,795 & ,497 \\
\hline & 4 & 8,750 & 1,241 & 61,683 & 0,29 \\
\hline & 5 & & & &, 178 \\
\hline \multirow[t]{5}{*}{ Estado Civil } & Viudo/a & & & & ,438 \\
\hline & Casado/a & ,635 & ,246 & 1,643 & ,349 \\
\hline & Divorciado/a & 1,200 & 0,95 & 15,196 & 888 \\
\hline & Separado/a & ,300 & 0,60 & 1,506 & 144 \\
\hline & Soltero/a & 1,091 &, 361 & 3,298 & 878 \\
\hline \multicolumn{2}{|c|}{$\begin{array}{l}\text { Lugar residencia (Urbano vs } \\
\text { Rural) }\end{array}$} & 1,368 & 630 & 2,971 & 429 \\
\hline \multicolumn{2}{|c|}{ Accesibilidad adecuada ( $\mathrm{Si}$ vs $\mathrm{No}$ ) } & 1,047 & ,533 & 2,055 & ,894 \\
\hline \multicolumn{2}{|c|}{ Propiedad vivienda ( $\mathrm{Si}$ vs No) } & 1,267 & 602 & 2,667 &, 534 \\
\hline \multicolumn{2}{|c|}{ Modalidad privada ( $\mathrm{Si}$ vs } & 1,119 & ,507 & 2,468 & ,780 \\
\hline \multicolumn{2}{|l|}{ Distancia } & ,999 & 997 & 1,001 & ,454 \\
\hline \multicolumn{2}{|c|}{ Jubilado (Si vs No) } & 2,095 & 1,020 & 4,302 & 0,44 \\
\hline \multicolumn{2}{|c|}{$\operatorname{Sexo}($ V vs M) } & 2,041 & 1,029 & 4,049 & 0,41 \\
\hline \multicolumn{2}{|c|}{ Riesgo social ingreso (No vs $\mathrm{Si}$ ) } & 1,360 & ,681 & 2,718 &, 383 \\
\hline \multicolumn{2}{|c|}{ DMellitus (Si vs No) } & ,629 & ,268 & 1,485 & 290 \\
\hline \multicolumn{2}{|c|}{ Hipertensión (Si vs No) } & ,980 &, 500 & 1,924 &, 954 \\
\hline
\end{tabular}

\section{DISCUSIÓN}

Se evidencia que la RHB integral tras el ictus tiene un impacto pronóstico que se mantiene a largo plazo, en forma de una mejor situación funcional de los pacientes que acceden a la misma. La recuperación funcional mediante RHB integral permite a los pacientes (entre otros efectos para su estado de salud), la permanencia en su entorno habitual, el desempeño de una actividad laboral y la capacidad para realizar actividades de la vida diaria por sí mismos, disminuyendo la necesidad de apoyos formales e informales por dependencia funcional y la institucionalización en centros residenciales.

Sin embargo, no todas las personas que requieren RHB integral logran acceder al tratamiento. Es una realidad que no existen recursos de rehabilitación funcional integral al alcance de todas las personas que son rehabilitables [6]. Y los que existen están sujetos a una diversidad de criterios de derivación a centros de rehabilitación basada fundamentalmente en aspectos personales, estructurales o económicos [13, 14]. Aún disponiendo de criterios objetivos de selección, el propósito de esta puede ser muy 
variable en función, por ejemplo, de la financiación disponible, de los recursos asistenciales existentes o su proximidad al domicilio del paciente, $\mathrm{u}$ otras prioridades personales/institucionales en base a requisitos muy diversos [15]. El acceso de pacientes potencialmente recuperables a ciertos tratamientos de rehabilitación integral públicos tiene, como criterio de exclusión, la edad. Ejemplos de ello son el Institut Guttmann y el Ceadac [6]. El lugar de residencia también supone una desigualdad en el acceso a la rehabilitación integral, por motivos tales como: la imposibilidad de desplazamiento del paciente al centro público dónde recibe la rehabilitación (por ausencia de medios de transporte y apoyo familiar para acudir regularmente, entre otros) y que la rehabilitación domiciliaria pública no se presta en todas las localidades donde residen los pacientes que cumplen los criterios clínicos para recibirla. Los resultados obtenidos muestran que el lugar de residencia (rural o urbano), la modalidad de tratamiento (sólo público o públicoy-privado) y Rankin al alta hospitalaria influyen en el acceso al tratamiento rehabilitador integral, que sólo resulta accesible al 53\% de los pacientes que lo necesitan. Constatando, por tanto, que estos condicionantes sociales y ambientales son marcadores de desigualdad en salud, tanto por el acceso diferencial al tratamiento rehabilitador integral post-ictus, como por los efectos que dicho tratamiento genera a largo plazo, en términos de autonomía funcional y esperanza de vida con calidad.

La equidad esté garantizada solo facilitando el acceso al sistema sanitario: hay que tener en cuenta las condiciones económicas, sociales y del entorno en que viven y se relacionan las personas. Lograr la equidad en salud requiere de un enfoque integral. El artículo 3 de la Ley General de Sanidad establece que el acceso a las prestaciones sanitarias se realizará en condiciones de igualdad efectiva y que las políticas de salud estarán orientadas a la superación de los desequilibrios sociales y territoriales. Algo que, en la actualidad y en el ámbito objeto de estudio, no se produce.

Otro estudio anterior, aunque mucho más limitado en tamaño muestral, objetivos y variables analizadas, identificó factores que influían en el acceso a RHB integral del paciente [6]. El presente estudio, además de contar con una muestra más amplia, incorpora la valoración de efectos a largo plazo del tratamiento rehabilitador integral en la situación funcional de los pacientes. También añade nuevas y diversas variables potencialmente relacionadas con el acceso a la rehabilitación integral, tales como el uso de tratamientos privados para la recuperación (con la capacidad de pago que eso conlleva por parte del paciente o su entorno), la propiedad de la vivienda, la situación laboral, o la distancia del domicilio al tratamiento rehabilitador, entre otras. Esta ampliación de variables se fundamenta en la recomendación del informe presentado al Parlamento Europeo, que indica que debe mejorarse el acceso al tratamiento rehabilitador [8].

Las recomendaciones del equipo investigador del King's College, en colaboración con Alianza Europea contra el Ictus (SAFE), que presentó el documento "El impacto del ictus en Europa" al Parlamento Europeo, señala que se deberían realizar evaluaciones multidisciplinares en la unidad de ictus y comenzar la rehabilitación en cuánto el paciente esté estable médicamente [8]. En nuestro estudio el abordaje multidisciplinar e integral se inicia desde el ingreso en Hospital de Agudos, y se recomienda el tratamiento rehabilitador integral post-alta hospitalaria.

Se ha demostrado que la RHB integral continuada desde el alta durante el primer año tras el ictus reduce el riesgo de deterioro funcional y mejora la independencia en las actividades de la vida diaria [16], pero los programas rutinarios de RHB en pacientes de más de un año de evolución no han demostrado una eficacia significativa [17]. Nuestra investigación aporta evidencias acerca de los efectos de un tratamiento rehabilitador integral a largo plazo. El programa rehabilitador no debe separarse, ni reiniciar tratamientos inadecuados [18] y/o disgregados por área funcional afectada, debiendo 
responder y englobar todas las áreas afectadas de la persona, de una manera integral. Esta dificultad en el acceso al tratamiento rehabilitador integral del paciente de ictus, es reconocida en el contexto europeo. En toda Europa hay una especial falta de terapia ocupacional, logopedia y fisioterapia [8]. El tiempo diario de rehabilitación (valorado por el equipo) que precisa cada paciente para obtener el máximo beneficio, no siempre está garantizado, tal como se ha puesto de manifiesto en el estudio CERISE [19]: la intensidad con que se realiza el tratamiento rehabilitador es muy variable según los diferentes países; la media de tratamiento diario es de $60 \mathrm{~min}$ en el Reino Unido, de $140 \mathrm{~min}$ en Alemania y de 166 min en Suiza. Además en toda Europa el apoyo y seguimiento a largo plazo continuos son insuficientes [8].

Esta falta de acceso al tratamiento rehabilitador integral individualizado y su seguimiento continuo a largo plazo, provoca falta de calidad de vida y un mayor gasto económico a la persona y a los Sistemas Públicos de Protección, además de evidenciar la inequidad en salud. Que solo un 53\% logre finalmente recibir una RHB integral, es indicador de la gravedad del problema de acceso que estamos tratando, sobre todo en lo relacionado con las barreras económicas (necesidad de complementar con tratamientos privados) y geográficas. Las publicaciones españolas más recientes sobre el impacto económico del tratamiento de los pacientes con ictus son los estudios CONOCES y CODICE. El gasto por paciente y año fue de 27.569 euros. Se observó que la mayoría de los costes $(67,3 \%)$ fueron los que se denominan costes informales, entendidos como la atención no pagada recibida por no profesionales, pero imprescindible para soportar las actividades de la vida diaria de las personas dependientes [3].

Los pacientes con ictus están en creciente aumento, los recursos destinados a la rehabilitación del paciente con discapacidad moderada-grave son extremadamente limitados en número y accesibilidad [20]. El acceso a la rehabilitación integral está condicionado no solo por variables clínicas, sino por variables sociales, como son la residencia en el medio rural y la ausencia o insuficiencia de ingresos económicos para asumir de forma privada los tratamientos rehabilitadores que no son cubiertos por el Sistema Público, pero que resultan imprescindibles para su recuperación integral. Por tanto, estos factores de desigualdad territorial y socioeconómica condicionan fuertemente el acceso a la rehabilitación integral y, con ello, la probabilidad de alcanzar una autonomía funcional a largo plazo.

Este estudio tiene ciertas limitaciones, derivadas de un diseño no randomizado. Se refiere a pacientes ingresados de los que una proporción muy reducida no fueron incluidos: se trata de algunos pacientes menos graves, cuya estancia hospitalaria fue inferior a tres días. Además de los que fallecieron durante el período de hospitalización y los que han fallecido a lo largo de estos 5 años. Por ello, el tamaño muestral es limitado. Se ha comenzado el estudio por los ictus isquémicos, por el especial interés que tiene este grupo, sometido a terapias que se han desarrollado en los últimos años, como el tratamiento endovascular. Pero en el futuro habrá que analizar el grupo de los hemorrágicos, que como es sabido, tienen aún peor pronóstico funcional. Otra limitación es que el pronóstico funcional está basado solo en el Barthel, por ser la escala utilizada al alta hospitalaria en 2015. Para asegurar la validez de los datos comparativos se empleó la misma escala en 2020 , pero en el futuro podrían utilizarse otras escalas de calidad de vida o medidas pronosticas aportadas por los pacientes.

El tratamiento rehabilitador integral es efectivo en la recuperación funcional de la persona, ya que se asocia a un mejor pronóstico a largo plazo de los pacientes con ictus. Los determinantes sociales generan una desigualdad en la recuperación de la persona con repercusiones significativas en el estado de salud. La gestión de caso social, propia del 
Trabajo Social, ha contribuido parcialmente a ampliar el acceso a la rehabilitación integral de pacientes que, por sí mismos, no hubieran accedido a los recursos movilizados por la trabajadora social. En este sentido, el Trabajo Social de neurología en la unidad de ictus, ha contribuido a la recuperación funcional a largo plazo de un mayor número de paciente, aunque con impacto limitado por los factores condicionantes que el estudio identifica. Se continuará investigando otros efectos sociosanitarios y laborales del tratamiento rehabilitador integral, tanto en los pacientes como en la comunidad.

\section{BIBLIOGRAFIA}

(1) Campbell BC V., De Silva DA, Macleod MR, Coutts SB, Schwamm LH, Davis SM, et al. Ischaemic stroke. Nat Rev Dis Prim. 2019;5(1):70. doi:10.1038/s41572-019-01188

(2) Romero JC, Llisterri JL, Turégano M, Cinza S, Muñoz L, Silvero YA et al. Características clínicas y sociosanitarias en mayores de 65 años asistidos en atención primaria. Estudio PYCAF. Medicina de Familia. Semergen 2019; 45(6). 366-374. doi: $\underline{10.1016 / \text { j.semerg.2018.10.004 }}$

(3) Noé-Sebastián E, Balasch-Bernat M, Colomer-Font C, Moliner-Muñoz B, Rodríguez Sánchez-Leiva C, Ugart, P et al. Ictus y discapacidad: estudio longitudinal en pacientes con discapacidad moderada-grave tras unos ictus incluidos en un programa de rehabilitación multidisciplinar. Rev Neurol. 2017; 64(9):385-392.

doi:10.33588/rn.6409.2016527

(4) Olmedo V, Arranz M. Trabajo Social Sanitario: Intervención con pacientes con ictus desde el Servicio Médico de Neurología del Hospital Clínico Universitario de Valladolid. Revista Trabajo Social y Salud. 2017;(88):39-48

(5) Kelly-Hayes PM, Robertson JT, Broderick JP, Duncan PW, Hershey LA, Roth EJ, et al. The American Heart Association Stroke Outcome Classification. Stroke. 1998;29(6):1274-80. doi: 10.1161/01.STR.29.6.1274

(6) ARTICULO CIENTÍFICO DE LOS AUTORES en revista indexada 2019 (ANONIMIZADO)

(7) Sociedad Española de Neurología. Plan estratégico nacional para el tratamiento integral de las enfermedades neurológicas. Madrid: Ibáñez Plaza Asociados; 2002.

(8) Murie-Fernández M, Laxe S. Neurorrehabilitación del ictus en España, comparativa con Europa. Neurología 2020;35:354-5. doi:10.1016/j.nrl.2018.02.007

(9) Millán Muñoz R. Modelos de rehabilitación ideal. [Internet]. Cali (Colombia): Fundación Ideal Julio H. Calonja; 2012 [consultado 17 de octubre 2020]. Disponible en:

https://www.fundacionideal.org.co/sites/default/files/publicaciones/archivos/modelorehabilitacion-integral-ideal2012.pdf

(10) Hong KS, Saver JL. Quantifying the value of stroke disability outcomes: WHO global burden of disease project disability weights for each level of the modified Rankin Scale. Stroke. 2009;40(12):3828-3833. doi: 10.1161/STROKEAHA.109.561365 
(11) Montaner J, Álvarez-Sabín J. La escala del ictus del National Institute of Health (NIHSS) y su adaptación al español. Neurología [Internet] 2006 [consultado 18 de septiembre 2020];21(4): 192-202. Disponible en: http://www.academia.edu/download/36160474/Escala_NIHSS.pdf

(12) López Espuela F, Portilla Cuenca JC, Leno Díaz C, Párraga Sánchez JM, GamezLeyva G, Casado Naranjo I. Diferencias de género en la calidad de vida a largo plazo tras un ictus: influencia del estado funcional y el estado de ánimo. Neurologia. 2020;35:470-8. doi.org/10.1016/j.nrl.2017.10.002

(13) Hakkennes S, Hill KD, Brock K, Bernhardt J, Churilov L. Selection for inpatient rehabilitation after severe stroke: what factors influence rehabilitation assessor decisionmaking? J Rehabil Med. 2013;45(1):24-31.

(14) Hakkennes SJ, Brock K, Hill KD. Selection for inpatient rehabilitation after acute stroke: a systematic review of the literature. Arch Phys Med Rehabil. 2011;92(12):2057-70.

(15) New PW. The Assessment and Selection of Potential Rehabilitation Patients in Acute Hospitals: A Literature Review and Commentary The Open Rehabilitation Journal. 2009;2:24-34.

(16) Legg L, Langhorne P. Rehabilitation therapy services for stroke patients living at home: systematic review of randomised trials. Lancet. 2004;363(9406):352-356. doi:10.1016/S0140-6736(04)15434-2

(17) Green J, Young J, Forster A, Collen F, Wade D. Combined analysis of two randomized trials of community physiotherapy for patients more than one year post stroke. Clint Rehabil. 2004;18(3):249-252. doi:10.1191/0269215504cr747oa

(18) Duarte, E. Rehabilitación del ictus: modelo asistencial. Recomendaciones de la Sociedad Española de Rehabilitación y Medicina Física, 2009.

Rehabilitación 2010;44(1):60-8. Doi:10.1016/j.rh.2009.10.001

(19) De Wit L, Putman K, Schuback B, Komarek A, Angst F, Baert I, et al. Motor and functional recovery after stroke: a comparison of 4 European rehabilitation centers. Stroke. 2007;38:2101-7.

(20) Vega Valdés P, Murias Quintana E, Morales Santos E, Cigarrán Sexto H, Larrosa Campo D, Benavente Fernández L, et al. Análisis de los costes directos asociados a la trombectomía mecánica y a la fibrinólisis intravenosa en el Hospital Universitario Central de Asturias. Rev Neurol. 2018;66(01):7. doi: 10.33588/rn.6601.2017208 


\title{
TERCER ARTÍCULO: LAS 4 “Q” DEL TRABAJO SOCIAL EN LA ATENCIÓN A PERSONAS CON ENFERMEDADES NEUROLÓGICAS
}

\author{
LAS 4 " $Q$ " DCL TRABAO SOCIAL rN LA ATrNCION A PLESONAS CON
}

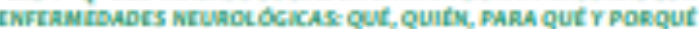

Las 4 " $q$ " del Trabajo Social en la atención a personas con enfermedades neurológicas: qué, quién, para qué y porqué

\author{
Modalidad: intervención profesional del Trabajo Social \\ Verónica Olmedo Vega \\ Trabajadora Social Saritaria en servicio midico de neuralogla \\ Sandra Fernández Diez \\ Trabuiadora Social fundación Secretariado Chano
}

Resumen:

El presente articulo muestra la aproximación al conocimiento profesional del tra bajo social sanitario que desempefia su intervención desde y con personas que sufren una enfermedad neurológica. Aunque la figura del profesional del trabajo soclal está consolidada en el campo de la salud, su inclusión en el campo de la neurologla es reciente y no existe una reguladón de las funciones, competencias, ni del perfil profesional. Labor, que esta por definir. El objetivo, visibilizar la necesidad de la especialización del trabajo social en la intervención con personas con enfermedades neu rológicas. Los cuatro plares fundamentales justifican su reconocimientoc el "que", definición y funciones, "quièn", "para que" y "porqué" de la misma.

El análsis de la experiencia de affos integrada en el área de neurología del Hospital Clinico Universitario de Valladolid muestra beneficios del conocimiento especializado frente a la formación generalista. Se logra una intervención social eficaz con el paciente al incorporar los dafios neurológicos producidos en la persona y su repercusid́n en su situación bio-psico-funcional condicionada por la evolución de la enfermedad. En la farmiliz, asesoramiento y apoyo soclal especializado, al equipo dónde se integra y a la profesión, les dota de legitimidad social e institucional.

\section{Palabras clave:}

Neurologia, Trabajo Soclal Sanitario, Intervención centrada en la persona, Enfer: medades Neurológicas, Intervención Soclal, Diagnóstico Social Sanitario, Salud, Caldad de vida.

\section{Summary:}

This article shows the approach to the professional knowledge of health soclal work that carries out its intervention from and with people who suffer from a neu rological disease. Although the figure of the social work professional is consolidated 
La intervención del trabajo social ante una enfermedad neurodegenerativa implica la incorporación de las caracteristicas de esa enfermedad. El abordaje peicosocial con la familila o un enfermo de Esclerosis Lateral Amiatrófica (ELA), que culmina con su fallecimiento, implica la presencia de este hecho, diferente al abordaje con una persona o familia de Al. zheimer, ambas son enfermedades neurodegenerativas pero las secuelas que provocan en la persona no son las mismas. Se requiere el disefio de una intervención social especifica con la persona y famlia que refleje el significado de la enfermedad que padece. El compromiso con una atención social adecuada y destinada al logro de bienestar de estas personas y famllas, exige, contribuir a la elaboración de un cuerpo teórico que garantice el apoyo social de la persona que sufre una enfermedad neurológica.

Este articulo pretende alcanzar el objetivo de visibilizar la necesidad de la especializa ción del trabajo social en la atención a personas con enfermedades neurológicas. Los re: sultados de la revisión bibliográfica desarrolada unido a la reciente investigación desde el trabajo sodial en el campo neurológico sobre los factores que influyen en la recuperación funcional de pacientes ictus junto con la sistematización de la experiencla profesional es pecifica del trabajo social sanitario en este campo evidencian la obligación de evitar centrar nuestra intervención en un modelo de intervención generalista. Se trazan en este artícula los mimbres hacia la especializadión en la materia a intervenir, las personas con enfermedad neurológica, que reporta numerosos beneficios a la persona famlia que se atiende y a la profesion que representamas.

\section{TRABAJO SOCIAL}

La atención sodal a personas que padecen una enfermedad neuroldgica es atendida por el trabajo social desde diferentes instituciones, es incuestionable, que el diagnóstico de la misma se produce en el hospital por el especialista en neurologla. En este imbito, aten diendo especificamente a las personas que sufren una enfermedad neurológica y dentro del equipo de neurologia, se encuentran profesionales del trabajo social sanitario.

Indiscutible, es el hecho, de que el trabajo sodial es una profesión que se nutre del tra bajo interdisciplinario, de equipo y de redes y que, a la vez, requiere de métodos cientificos de investigación para mejorar los procesos de intervención de las personas y su entomo. Los limites de acción del trabajo social son amplio y diversos, alcanzando todas las esferas donde están presentes las relaciones sociales. Un ejemplo de ello lo encontramos en su presencia, cada vez mayor, en ámbitos como la politica, el derecho y el sanitario entre otros. En el caso de los espados de salud, el trabajo social Sanitario adquiere un importante papel con las enfermedades neuroldgicas.

El trabajo soclal sanitario es la actividad profesional que tiene por objeto la investigación de los factores peicosociales que inciden en el proceso de salud = enfermedad, asi como el tratamiento de los problemas psicosociales que padecen en relación a las situaciones de enfermedad, tanto sil influyen en su desencadenamiento como derivan de elas. (Ituarte, 2001 en Agencia Valendana de Salut (2012).

El trabajo social sanitario es la especialidad del trabajo social que se desarrolla y se ejer: ce dentro del sistema sanitario, ya sea los diferentes niveles de la atención primaria, la 
atención sociosanitaria o la atención especializada. "La intervención desde el trabajo social sanitario siempre toma como punto de partida la enfermedad que sufre la persana, el diagnóstico médico que motiva su consulta sanitarla o, induso, el ingreso hospitalario, su vivencia" (Coloms 2010, p.no). Bonilla darifica este enunclado como: "el trabajador social aporta su experiencia en la valoración de la dimensión social, famillar y peicosocial, elaborando el diagnóstico social sanitario que complementa el diagnóstico clínico" (2017, p. 8.2). El traba/ador social en el ámbito de la salud persigue "movilizar capadidades y recursos de la persona afectada y de aquellas que forman parte de su red social, así como promover cambios que garanticen su calidad de vida y hacer posible el abordaje adecuado de su situa: cion personal" (Olmedo y Arranz, 2017, P. 41).

Este profesional, es el enlace entre el ámbito sanitario y las personas, entre la enfermedad y la rehablitación.

\section{TRASTORNOS NEUROLÓGICOS}

La Organización Mundial de la Salud expone que "Los trastomos neurológicos son enfermedades del sistema nervioso central y periférico, es decir, del cerebro, la médula espinal, los nervios craneales y periféricos, las raices nerviosas, el sisterna nervioso autónomo, la placa neuromuscular, y los músculos" (OMS, 2016, pairr.2).

El cerebro es una de las estructuras mas extraordinarias y complejas cue existen en la naturaleza. A

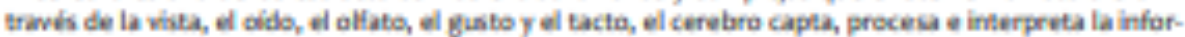
mación que lega del estericr, gener ando las respuestax que originan pensamientos, razconamienton, decisiones, serntimienten y acciones. En el cervbro reside todo lo que una persona fue, es y puede llegar a ser, lo que ha vivido, aprendide y memorisado, su canciencia y su metaconciencia (eraidot, 2003, PP. Q-10)

Es indudable que el alcance del dafio que ocasiona una enfermedad neurológlica unido a la cada vez mayor presencia de ésta en nuestra sociedad, la existencia de más de 600 enfer: medades neurológicas, entre ellas, el Alzheimer, Parkinson e ictus, implica la capacitación del trabajo soclal sanitario en neurologla para una atención e intervención soclal de calidad.

\section{TRABAUO SOCIAL EN LAATENCIÓN A PERSONAS CON ENFERMEDADES NEUROLÓGICAS}

\section{L. ¿Quées?}

La atención e intervención social con personas con enfermedades neurológicas es com: petencia del trabajo social que desarrolla su actividad en el campo de la neurologia. Se podria decir, por tanto, que el trabajo social en el campo de la neurología es una especial: zación del trabajo sodial (Olmedo y Conzilez, 2019) y requiere de una formadón especifica acreditada. El trabajo social en neurologla tiene como principales objetivos la detección de problemáticas sociales y el abordaje integral de la situación sociosanitaria del enfermo desde su ingreso hospitalario, establediendo planes de posthospitalización que facliten el soporte necesario para seguir las indicaciones terapeuticas y para mantener una calidad de vida optima tanto en el enfermo como en su principal apoyo, la familia.

[10] Documentas de Trabojo Social / Ir63 - 155N 1133-6552 / ISSN Electrónica 2073-8246 
LAS 4"Q" DCL TAABAO SOCIAL TN LA ATrNCION A PRR SONAS CON.

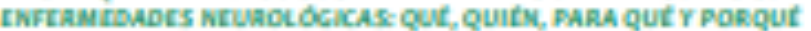

\subsection{Funciones del trabajador social con personas con enfermedades neurologicos}

El profesional de trabajo social debe conocer al ser humano para adquirir la capacidad de comprender sus conductas y atender las demandas que las alteraciones de salud le producen. Es un elemento clawe en todo el proceso de la persona, su familia y entorno propor: cionando orientadón y acompafiamiento social en el transcurso. En el ámbito hospitalario aborda el impacto del diagnóstico, el acompafiamiento social durante su ingreso, el afrontamiento a la nueva realidad a la que ahora se enfrenta unido a la angustia que produce la incertidumbre de un futuro desconocido.

Si bien es clerto, que se defiende como dentro del campo de actuadón del trabajo social sanitario con personas con enfermedad neurológica, este no solo ha de conocer a la persona, sino también las distintas enfermedades, dlagnósticos, secuelas y aquellos factores significa: tivos que incurran en este campo, fundamental para abordar las funciones que se detalan a continuación, siguiendo el articulo de Olmedo y Arranz (2017, pp. 42.43), se sefialan, algunas de las funciones en el campo en la intervencidn con personas con enfermedad neurológlica:

- Asesorar, acompanfar y propordionar apoyo sodalt Es el profesional de referencia al que ba familia y red de apoyo recurren para pedir orientación social cuando sea necesa. rio; proporciona una información realista y veridica al afectado y su famlila en todo momento. Ofrece el acompafiamiento social a la persona que lo sufre y su familia durante ef ingreso hospitalario, alternativas a las incidencias y conflictos que surgen durante ef mismo. Elabora la anamnesis social de b situación personal, detección de los factores de indole social en el entorno de la persona que influyen negativamente en el estado de salud de éstos o su famllia. En el ámbito hospitalario se encarga de proporcionar apoyo y tratamiento soclal a la persona y su familia desde el ingreso hospitalario, realzando la gestión y coordinación de las actuaciones precisas para el logro del mayor bienestar posible de la persona y famlila.

- Gestión de recursos; búsqueda, organización y creación de los mismos: La detección de las necesidades de la persona o familia para orientar al acceso de los recursos que las cubran, conoce los recursos existentes en el hospital y en otros Sistemas de Protecdón Social. El conocimiento, asesoramiento y protocolo de acceso de todos los recursos exis. tentes para personas con trastornos neurológicos, sus caracteristicas, ubicación, tratamiento, objetivos y finalidad, entre otros. Es evidente que, por motivos variados, ciertos tratamientos o recursos que precisa la persona no son accesibles para éste, el trabajador social sanitario de neurologla promoverá o propondrá la creación de los mismos.

- Ser nexo de unión en y dentro del entorno hospitalario, entre los miembros del equipo multidisciplinar que atiende a la persona, la familia y el equipo médico, con otros hospl. tales cuando sea necesario. Es interlocutor entre los distintos profesionales, faclitando el flujo de información y la retroalmentación entre los diferentes agentes involucrados en la atención a la persona.

- Comunicación inter e intrainstitucionalt Promueve espacios comunes de comunicación para darficar posiciones, conseguir consensos y sugerir pautas de funcionamiento que contribuyan a la atención integral de ba persona afectada. Desde su abordaje psicosoclal promueve una valoración global de las necesidades de la persona, trabajar de forma coordinada y conjunta con el equipo para faciltar la atención que precisa esa persona. 
Este profesional deberá tener capacidad para coordinarse con otros recursos asisten dales, creando protocolos y rutas de derivación haciendo que el proceso de hospitalzación sea mís fluido y iglil, desarrollando un trabalo proactivo donde se confeccionen registros de las personas afectadas por la enfermedad neurológica y se recolan factores soclales y patrones que ayuden a anticipar la atención para amortiguar el impacto de las enfermedades.

- Promoción y desarrollo de espacios de liberacióna Promueve encuentros con la persona y famlila que contribuyan a su ventlación emocional, a escuchar las demandas y detec. tar las necesidades que ayuden a proporcionar apoyo emocional y la expresión de emadones, promoviendo así la normalización de sentimientos, preocupaciones y miedos.

- Se apoya en el duelo de los familiares y de la persona para afrontary adaptarse a las nuevas situaciones. Los refuerzos positivos a los involucrados que prestan la atención están presentes en estos espacios. Creará un espacia libre de ventilación emocional, donde se expresen las preocupadones, temores, la tristeza o la soledad, así como de realizar un plan de trabajo, aportando junto con el médico y la enfermera, el diagnóstico social sa nitario e implementando programas que permitan traba/ar el impacto de la enfermedad a todos los niveles (farniliar, social, laboral, escolar, entre otras).

- Proporclonar una mirada optimista y luchadora que ayude a empoderar a las personas y mantener la esperanzas identificar con la persona sus limitaclones, clarificando con esta las actividades que puede desempefiar y que apoyos la complementan. Se trata junto a ella, el cambio en la autoimagen y la construcción de nuevos valores que fruto del impacto de la enfermedad, aparecen o se han visto dahiados e influyen en su calidad de vida y bienestar. Promover y resaltar sus fortalezas y los logros conseguidos con la rehablitación durante y tras la estancia en el hospital. Se refuerza el esfuerzo, motivación y el tesón de la persona afectada. A la famlila y persona afectada se transmite calma y tranqulidad durante toda la intervención.

Como podemos ver, durante el proceso de atención del profesional del trabajo social sanitaria/a se realiza un apoyo integral, a las familas y persona, quienes se enfrentan a la dolorosa aceptación de las consecuenclas que ha provocado esta patología en su realidad. La forma en la que las famlilas tienen para elaborar el duelo generalmente sigue unas pautas que son unlversales: desorientación, regresión, reorganización y crecimiento.

La intervención y funcianes del trabajo social va a depender del momento en la que se encuentre la persona y su familia. La diferencla en la intervención, respecto a otras profesionales de trabajo social sanitario, radica en que el desempefio de las funciones anterior. mente descritas se realizan sobre una persona con una enfermedad concreta del sistema nerviaso, central ylo periférico, lo que resulta determinante para una intervención social, es decir, las personas afectadas por algún tipo de enfermedad neurológica, sufren algún tipo de deterioro cogritivo o afectación en diferentes áreas: motora, lenguaje, emocional, entre otras, y por tanto, que determina y condiciona el plan de intervención social.

La intervendón social del profesional de trabajo social en el campo de la neurologia en muchas ocasiones deja de ser un modelo centrado en la persona y se comierte además en una atendón con y para la persona, que ahora es, esa persona tiene un deterioro cogritivo tan afectado que no es la misma, ni puede expresar sus deseos, ha perdido su identidad.

[12] Docamentos de Trabajo Social / n'63 - ISSN 11336552 / 15SN Electrónico 2173-8246 
En otros casos en los que las personas afectadas por enfermedades neuroldgicas, como caso de la ELA, preservan la capacidad coginitiva hasta su falecimiento, pero no el lenguaje, teniendo que adaptar la entrevista y el acompafiarniento con el manejo de herramientas especificas que permitan comunicarse con la persona.

\section{3. ¿Quién es?}

El desarrollo de las funciones anterionmente descritas, exige que el profesional en dicho servicio tenga conocimientos especificos acreditados, entre otros, conocer la diferente ter. minologla clínica, pruebas diagnósticas, factores de riesgo, la estructura y función del cerebro, funcionamiento de un servidio clínico de neurologia, conocimiento y entendimiento de los distintos profesionales que le conforman, el dominio de protocolos y gufas de inter. vención sabre las diferentes patologias, ademís de las competencias concretas en técni. cas propias y especificas del trabajo soclal en el área de neurologla, escalas de valoración y fundamentalmente participadión en grupos cientificos, publicaciones e investigaciones que contribuyan a la evidenda dentifica de la atención social de las personas en esta especialidad (Olmedo y Conzález, 2019).

\subsection{Perfil del trabajo sodal en el campo neurológica.}

El perfil profesional es una herramienta vital, que garantiza las rasgos particulares o especificos que se deben poseer para el desempenfo correcto de funciones y tareas de una determinada profesión. El conocimiento especifico, experiencia, habelidades, destre 2as, entre otros. Olmedo y Aguado (2019), en el articulo "El abordaje psicosocial con pacientes que han sufrido ictus" mencianan la necesidad de tener conocimientos especificos del campo dónde se va a intervenir. La elaboración e implantación de un perfil concreto con unos conocimientos y competencias definidos del trabajo social en el campo de la neu rologla, es imprescindible. Se detalla a continuadón una propuesta:

\section{Estar en poseslón de conocimientos especificos relaclanados con:}

Las diferentes enfermedades neuralógicas, como kctus, ELA, Esclerosis Máltiple, Demen cias y Alzheimer, entre otras; secuelas, sintomatologia, terminologia clinica, afectación, pronóstico dínico de recuperación, su epidemiologia, etlologia y tratamiento, así como el conocimiento de la explaración neuralógica, procedimiento y pruebas diagnósticas, el conocimiento de los diferentes códigos y procedimientos de actuación como puede ser el Codigo ictus.

El funcionamiento del servicio médico de Neurologla, su estructura organizacional y or: ganigrama, el conodimiento de los distintos profesionales que trabajan en el servicio, dominio de los protocolos y gulas de intervención asociadas a la patología neurologla, entre otras.

Los recursos y apoyos especificos destinados a personas que han sufrido o padecen una enfermedad neurológica (requisitos, finalidad, objetivos, trámites a realizar, tiempo de duración, ....), aquellos recursos de sistemas de protección social (Servidos Sociales, de 
Educación o Empleo...) y los diferentes movimientos asoclativos dedicados al apoyo a las personas con enfermedades neuroldgicas.

Relacidn y contacto con la comunidad, representando la capacidad para detectar riesgos psico-sociales que permitan intervenir en su comunidad y ser una conexión con el medo, que permita una comunicación fluida y continua con las diferentes Asoclacianes de Familiares y pacientes.

\section{Poseer competencias concretas en:}

El manejo de Técnicas y herramientas propias y especificas del trabajo soclal con persanas y famillares como, las dferentes escalas de valoración a familiares y personas afectadas, técnicas de duelo y afrontamiento ante la pérdida y el programa informático de registro de los profesionales del Servicio Médico de Neurologia que intervienen con la persona.

La creación y dilrección de un equipo de trabajo donde se diseffen los proyectos de atendón grupal para familiares y personas afectadas de una enfermedad neurológica, hav dendo respetar los valores y creencias de la persona, asl como el respeto a su intimidad, confidencialidad y a los derechos de las personas usuarias del sistema de salud.

Participacib́n en grupos cientificos de enfermedades neurodegenerativas y realizar trabajos de investigación sobre aspectos sodales o de intervención del trabajo social en el campo de la neurología.

En definitiva, el Trabajo Soclal que interviene con personas con enfermedades neuroldgicas, ademis de las capacidades, habilidades y actitudes de empatía, intervención exenta de julcios, han de poseer conocimientos especificos para dar una atención soclal que dé respuesta a las necesidades que provocan en la persona, farnilia, equipo multidisciplinar y sociedad las diferentes enfermedades neurológicas. El logro de la calidad y bienestar en la persona y su familia que guia al profesional de trabajo social en el campo de la neurologla implica incorporar el significado de esa enfermedad, que genera o va a generar en ellos.

\section{5. ¿Por que?}

La intervención profesional del trabajo soclal con personas que padecen estas enfer: medades debe empezar por, poseer un grado adecuado de familiaridad con la patologla de interés, así como de los diferentes factores modificables y no modificables que influyen en la aparición y tratamiento de las mismas. A continuación, se exponen algunos de los motivos por los que se apuesta por una especialización del trabajo social en el campo de la netrologia:

En 1866, Rilliet y Barthez ya establedan en su "tratado clínico y príctico de las enfermedades de los nifios" la necesidad de conocer la naturaleza y condiciones fisiológicas de las distintas afecciones, ya que, en este sentida, se aseguraba una mejora de la atención, que pudiera levar a graves consecuencias para las personas afectadas por estas enfermedades.

[14] Docummtas de Trabojo Social - n't63 - 155N 1139-6552 / ISSN Electrónico 203-8246 
Izquierdo (1968), sefíaló que las ventajas de conocer la etiologia y las diferentes manifestaciones de su desarrollo y perpetuidad marcan la relevancia de conocer la patologia de una enfermedad. Otro ejemplo que podrlamos citar, y ya referente al campo de las enfer: medades neurológicas como es el Alzheimer, Martinez y Miangolarra nos hablan, de "la importancla de conocer y saber identificar los puntos clave de las diferentes situaciones clínicas referidas a esta enfermedad, y que puede trasladarse a otras enfermedades neurológicas, que, a la larga, se traducen en un gran deterioro para la salud de las personas, sin unos conocimientos adecuados" (2006, p.12). Estos tratados ya ponen en alza, que, desde hace más de 100 ahos, la especificidad a la hora de intervenir de forma clínica, debía ser un requisito indispensable para todos los profesionales del ámbito de la salud.

De acuerdo con los estíndares éticos de la APA (estaindar 2.01a b), solo se deberian aceptar casos que estén dentro de nuestros conocimientos profesionales. En caso contrario, es útil preguntar a colegas expertos que caen fuera de nuestras competencias (Del Barrio, 2018).

Rodriguez y Carcla (2019) ponen en alza, que la creación de programas de postgrado es: pecificos en diferentes universidades favorecerla la visibilidad de profesionales e investigadores especializados y, por tanto, un mayor número de docimentos cientificos al respecto.

Los profesionales del trabajo social en el campo de la neurologla deben realzar un diag: nóstico social de la situación para definir, posteriomente, la mejor ruta de actuación, que tenga en cuenta las particularidades de la enfermedad y lograr una intervención social de calidad.

Como podemos ver, la demanda de una mayor especialidad en los diferentes ámbitos del trabajo social, sobretodo en el sanitario, no es algo nuevo, pero si poco visibilizado. Es nuestro deber promover, en el ámbito académico y profesional, una reivindicación de la especialización y abogar por un proceder que supere la mera gestión y coordinación de recursos (Cijón y Colom, 2016), y por la transdisciplinariedad.

Si nos fijamos en las siguientes notidas, podemos observar la evidenda de como las enfermedades neurológicas están evolucionando y no afectan solo al nivel físico de la per: sona, sino que los familiares y amigos de la persona se ven también afectados, asi como las importantes consecuencias socioeconómicas que traen consigo.

Según datos de la Sociedad Espafiola de Neurologla (SEN), la Esclerosis Múltiple ' (EM) registraba en 2018 a 47.000 personas afectadas en Espafia y es, tras los accidentes de tra. fico, la principal causa de discapacidad en jóvenes. Cada af́o se daginostican L8̊oo nuevos casos en Espafía de esta enfermedad que afecta a 700.000 personas en Europa y a 2,5 millones de personas en todo el mundo (Organización Médica Colegial Espatiola, 2015).

Continuando con lo citado anteriomente, de que las enfermedades neurológicas no afectan solo a la persona sino también a su desarrolo y medio, el informe sobre; "El impacto socioeconómico de la EM sobre las mujeres en Europa de $2017^{\prime \prime}$ 'hizo públicas sus conclusiones en la jornada del VII Congreso ECTRIMS-ACTRUMS de Paris (octubre del 2017) cuyos

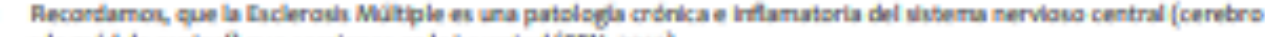

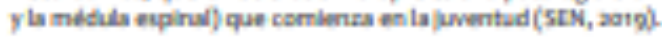


resultados evidenclan el gran impacto que la EM juega en la vida de las mujeres, sus familias y su papel en la sociedad. En dicha conferencia, Kannan, Senior VP y responsable global de Neurología e inmunología del área de Biopharma de Merck explicóc "La evidencia muestra que, dado que las mujeres tienen mayores posibilidades de recibir un diagnóstico de EM en comparación con los hombres, la carga especifica de la enfermedad en ellas requiere una mayor investigación y entendimiento" (Salud a Diario, 2017, parr. 5). Este informe de impacto asegura que el 418 de las mujeres diagnosticadas de EM termina separfindose o diworclándose de su pareja, evidenciando, que la enfermedad impacta seriamente en otras decisiones que toman respecto a su vida.

Otro ejemplo que citamos en el articulo y quizás, la afección neurológica mís comán, es el de las cefaleas y migrafias, conceptos que suelen confundirse aunque son dispares. El témmino cefalea significa, sencllamente, "dolor de cabeza", mientras que Migrafia o laque $\mathrm{ca}$, hace referencla a un tipo concreto de dolor de cabeza con unas caracteristicas clínicas concretas. Como podemos observar, que, aunque distintas, las cefaleas y migrafias afectan a gran parte de la pobladión y tienen repercusiones en la salud y vida de las personas. Sin embargo, debido a su al ta prevalencia, podemos decir que la migrafia tiene más repercusión social y laboral, ya que suele darse en los afios medios de la vida de una persona, es decir, los más activos y productivos, y que dependiendo de su naturaleza y como afecte a la persona, su manifestadón puede dejar a quien lo sufre incapacitada temporalmente para realizar acclones de la vida diaria y cotidiana, lo que influye en su vida laboral y social (SEN, 2017).

\section{6. ¿Para que?}

La necesidad imperiosa de un amplio conocimiento de estas enfermedades por el profesianal de trabajo social es fundamental en todas las intervenciones, se torna vital en las personas afectadas por estas patologias.

El campo de la neurologla y la especificidad de las funciones del trabajo social, a diferen cia de otras especial idades que tratan la enfermedad desde el contexto físico de la persona; como pueden ser; traumatologla (fractura de cadera, rodlla,...), neumologla (problemas respiratorios, deficiencias cardiacas, neumonla,...), tratan y estudian las enfermedades que afectan al cerebro, un órgano cuyas afecciones no alteran solo al área motora, sino tambien a la cognitiva, lenguaje, y psicológica de la persona. El trabajo social que interviene con personas afectadas por una enfermedad neurológica debe poseer desde el iniclo de su intervención con la persona y famila, conocimiento sobre la enfermedad neurológica que padece. Se resal ta la complejidad en la intervención social, adaptada no solo a la situación individual, famillar y social de la persona sino a los efectos de diagnóstico y pronóstico del mismo.

Es indudable que, si somos capaces de identificar las diferentes enfermedades neurológicas, como et ELA-esclerosis múltiple o ictus, podremos intervenir con la persona y ver su nivel de funcionalidad y condencla para lievar a cabo un determinado tipo de actuaciones. En enfermedades neurodegenerativas que finalzan en una muerte anunciada, como el caso de Ela, el conocimiento especifico de este profesional, le orienta para acompafiar a sus familiares y amigos en el proceso de duelo, posiblitar los apoyos necesarios para en sef̂ar a vivir con la pérdida y adaptarse a la nueva realidad, evitando dafios en su persona.

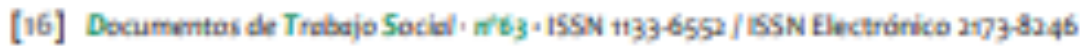


"La enfermedad de Azheimer (EA) es la causa mis frecuente de demencia, es un proce: so neurodegenerativo que afecta preferentemente a mayores de 65 ah́os, ocasionando en la mayoria de los casos peirdida progresiva de un tipo muy selectiwo de demencia" (Navarro, Conde y Villanueva, 2015, p. 4306). Existen factores de riesgo, de predisposición y adquiri. do, tanto dependientes del propio individuo como de su entorno. El nivel de formación, la actividad mental, el aprendizaje y la interacción social contribuyen al mayor desarrollo de las actividades cognitivas que disminuyen su impacto. (Navarro, et al., 2ons)

Son por estos motivos, entre muchos otros, que se constata b especificidad en este campo, para garantizar que el trabajo social siga manteniendo un papel preponderante en la gestión de casos, al conocer del funcianamiento de los sistemas sanitario y social, y de la enfermedad contextualizada (Carcia Bustelo, 20n5). El profesional del trabajo social debe tener conocimiento en este campo, primordial, para, ser capaz de valorar el estado y funciones de la persona atendida, saber identificar e intervenir de forma rápida sobre las enfermedades, contribuir a evitar la asoclación de problemas de salud no deseados que agraven y aceleren la perdida de bienestar y funcionalidad que prowocan este tipo de pa: tologlas. Se colabora en la prevención de una evolución ráplda de la enfenmedad, puesta en marcha de medidas de atención de los factores de riesgo modificables por la persona, aglidad en el proceso de intervencid́n y orientaciones para la adherencia del tratamiento. La especializadión en este campo es necesarla, para establecimiento de programas de in: formación social en personas y familias que faciliten la adquisición en ellos de pautas para buen culdado y atención de la enfermedad que padece.

Se incluye más abajo una tabla con algunas de las enfermedades neurológicas que pa: decen personas, llustra los posibles culdados y áreas afectadas que ha de incorporar ef trabajo social sanitario para garantizar el éxito en la atención social. Es importante tener en cuenta, que las posibles secuelas de las enfermedades neuroldgicas van a afectar a la autonomla y vida de las personas, limitando en muchos casos aquela e impidiendo realizar de forma adecuada o total, las Actividades Básicas de la Vida Dlaria o cotidlana de la persona (en adelante, ABVD). En la siguiente tabla se aportan ejemplos para reflejar este hecho.

Tabla L. Cuidados y áreas afectadas según la enfermedad.

\begin{tabular}{|c|c|c|}
\hline Entermedad & Arean alectadan & Penlbles caldaton y necenidates \\
\hline Wathelmer & 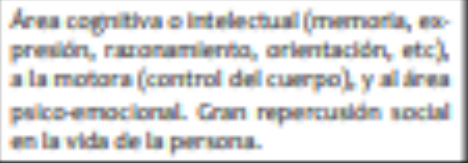 & 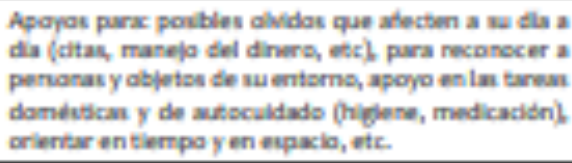 \\
\hline Ipllepula & 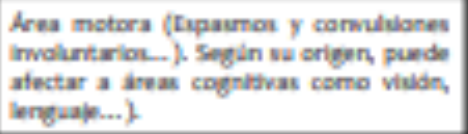 & 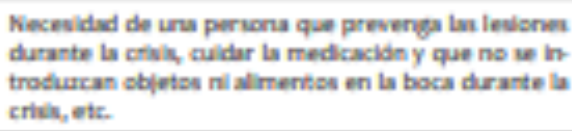 \\
\hline $\begin{array}{l}\text { Cefaleat o } \\
\text { migrafim }\end{array}$ & 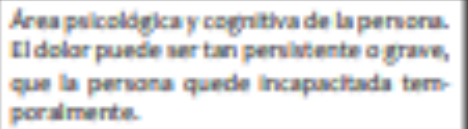 & 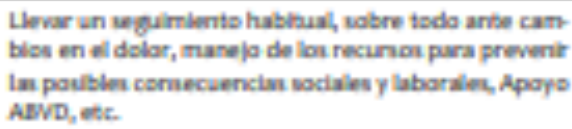 \\
\hline
\end{tabular}




\begin{tabular}{|c|c|c|}
\hline kctur & 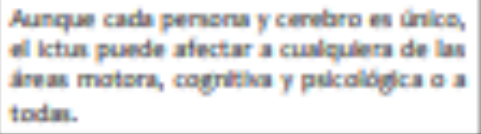 & 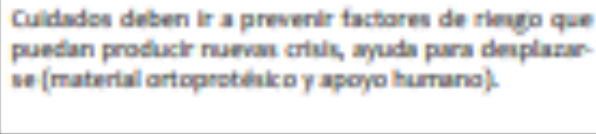 \\
\hline $\begin{array}{l}\text { La Tecleronin } \\
\text { multiple ([CM) }\end{array}$ & $\begin{array}{l}\text { Area cegnitiva, motora y canductual de la } \\
\text { pernonk. }\end{array}$ & 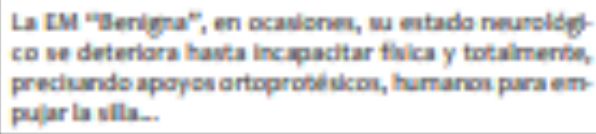 \\
\hline Parkinion & 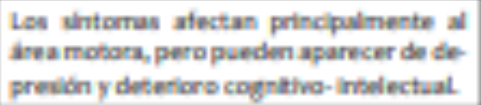 & 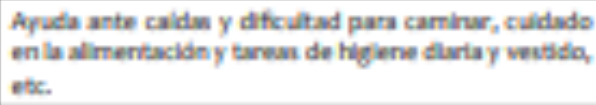 \\
\hline $\begin{array}{l}\text { La Lefermedad } \\
\text { Corea de Hant- } \\
\text { Ingtan (cin) }\end{array}$ & 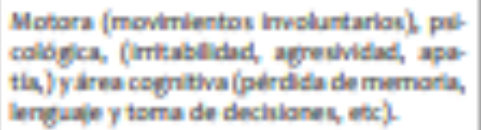 & 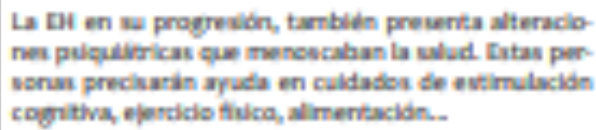 \\
\hline
\end{tabular}

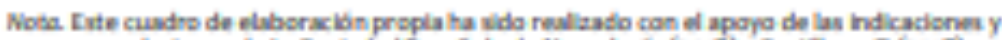

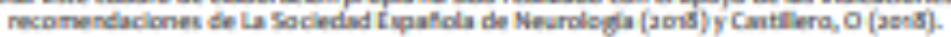

\section{FASES DEL PROCESO dE INTERVENCIÓN CON PERSONAS CON ENFERMEDAD NEUROLÓGICA}

Este apartado ha sido elaborado a ralz del trabajo de Olmedo, V. y Arranz, M. (2017), en su articulo sobre el Trabajo social sanitarios Intervención con pacientes con ictus desde ef Servicio Médico de Neurología del Hospital Clínico Universitario de Valladolid, de las consideraciones de Antonio del Barrio (2018) y de Cordon y Hamilton (en Colom, 2011).

\section{PRIMERA FASE. Recepción del caso.}

Al ingreso de la persona en el Servicio Médico de Neurologla del hospital se comunica al profesional de trabajo social saritaria, que inicia el proceso de atendión social. Las cir: cunstancias y necesidades sociales particulares determinan las primeras actuaciones, que se orientan en la localización de familiares o referentes de la misma, de su documentación identificativa, organlzar las ditas para una peimera entrevista con las familias o referentes y comunicar la información obtenida al equipo del servicio de neurologia.

En esta fase comienza el acompafiamiento soclal del profesional a la persona y su familla.

\section{SECUNDA FASE. Exploración y estudio sociofamillar.}

El personal del trabajo social en el campo de la neurología explora y aborda el conod: miento amplio de la situadión sociofamiliar (composición y dinaimica famlliar, comunicación, capacidad de autonomla y decisión, apoyos reales con los que cuenta, entre otros)

A través de técnicas $\mathrm{e}$ instrumentos propios del trabajo social, se realiza una exploración social estructurada y adaptada al estado de salud de la persona. El objetivo es conocer la situación, sus necesidades y deseos, los apoyos que va a ir precisando. Como soporte 
LAS 4*Q" DCL TaABAO SOCIAL RN LA ATrWCION A PRESONAS CON rwFramrouders nruaclocicas: qut, Quits, para qut r ponqut

documental, se abre a cada persona su historla social, custodiada en los archivos de este profesional.

\section{TERCERA FASE. Valoración social.}

En esta fase se definen y concretan las factores que inciden en la situación de necesidad/ problema psicosocial de la persona y su entorno, que orientará el plan de intervendín.

El objetivo es establecer el diagnd́stico social riguroso que permita planificar una inter: vención eficaz. El diagnóstico social equivale, para el trabajo social lo que el diagnóstico médico significa para el personal médico. En el diagnóstico, sea médico o sodial sanitario, se apoya todo el tratamiento (Colom, 2010, p.n3). Es importante recoger los codigos diag. ndsticos de la Clasificación Intemacional de Enfermedades y Problemas Relacionados con la Salud (CIE, no). La valoración social realizada, está condicionada por la afectadión y extensión de la enfermedad, sujeta a cambios segin la evolución de la misma, ha de reflejar, las direas afectadas en la persona, el pronóstico, si existe posibilidad de recuperación y en qué áreas, conocimiento y percepción de la persona y familia ante el ictus, el tratamiento clínico social establecido, ayudas técnicas y apoyos sodales que precisa al alta. Se aplican, escalas y cuestionarios, que valoran aspectos clínicos, aspectos relacionados con la vida diarla de la persona y su entomo que han permitido establecer el diagnóstico social sanita. rio y en base al mismo, establecer el plan de intervención social.

A continuación, para faclitar la comprensión del contenido, se recoge en una tabla algunos de las instrumentos que se utiliza en las procesos de valoración de be persona:

Tabla 2. Herramientas de valoración.

\begin{tabular}{|c|c|}
\hline Cl cuentienarlo te alud 5 -t2. & 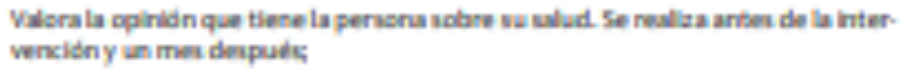 \\
\hline La Tucala de cajbn. & Viera ell ahtema seclo tamilur. \\
\hline Cucala Luwtan y larady. & $\begin{array}{l}\text { Dvala la a.tonemia fivica y lan actividades intrumentalea de la vida diaria de la } \\
\text { pernana. }\end{array}$ \\
\hline Cuestlararis Mas. & Mueutra el apoyo emocional, materid, socid y afectivo eque poiee la peruona. \\
\hline Cuestlonaris Apgar tarnillar. & Debecta elutancianes en dimbits tarnilur. \\
\hline Cucalazarit & Bermite canocer el grado de actrecarga del culdador. \\
\hline $\begin{array}{l}\text { Cuestlonarle Dake o de apeyo asclal } \\
\text { perclbida. }\end{array}$ & Revela la red social are posee el ind ividac. \\
\hline Cecala larthet & Permite obbener una valoracidn funcional de la pervoru $y$ w wh hablexidn. \\
\hline Cucala de Beck. & Bermite detectar ai la pernana satre depresidn. \\
\hline
\end{tabular}

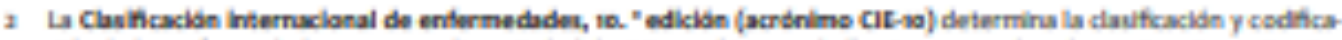

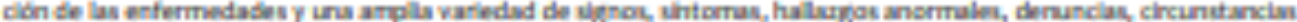

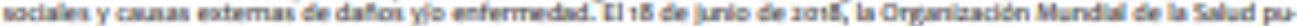

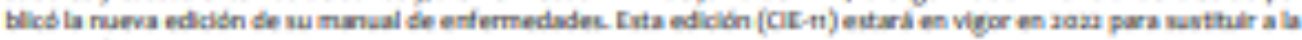
vigente de spos. 


\begin{tabular}{|c|c|}
\hline 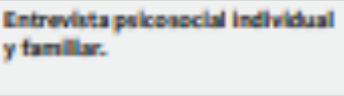 & 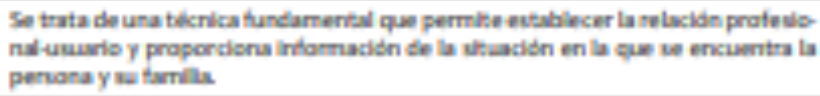 \\
\hline Obeervadidn. & 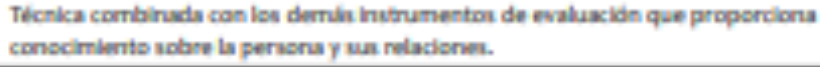 \\
\hline Cenagrama e hixtegrama. & 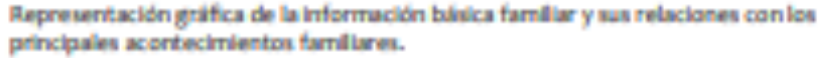 \\
\hline
\end{tabular}

Neta. La vipuente tabla de elaboracidn propla ha vide realtrada a rale de la informacidn extraida del articulo de Clmedo y Arranz ( $a s 0$ ) y de la apertacianes de las auteran de evbe trabuja.

Toda la información se registra en el programa informático dónde el trabajo social de neurologia desempefía su labor.

\section{CUARTA FASE. Plan de intervención social.}

El plan de intervención social recoge objetivos, áreas, actuaciones a desarrollar, se ejecutan un conjunto de acciones que garanticen la atención que precisa la persona durante su ingreso hospitalario y al alta del hospital, logrando la recuperación de su autonomía e independencla psicosocial del mismo, siempre condicionados por la evolución de la enfer: medad.

Es decir, este plan de intervención está caracterizado por la adaptadón individualizada a la situación de cada individuo, partiendo de la intervención centrada en la persona, la persona es en cada momento lo que la enfermedad le prowoca (Falta de conciencla, imposibilidad de comuricarse, entre otros).

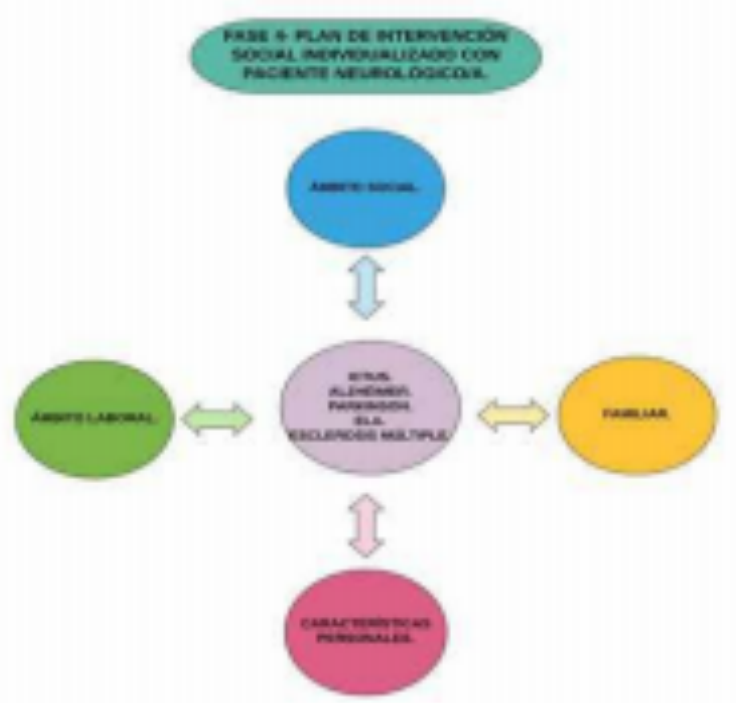

liustración del plan de intervención. Grafico de elaboración propia.

[20] Docummtes de Trabojo Socid ' n'63 + 15SN 1993-6552 / ISSN Electrónica 2179-8246 
El logro de una intervención social eficaz en la atención a estas personas está condicionado por la enfermedad que padece. Se atiende a la persona y los ámbitos en los que desarrolla su vida que influyen y son influenciados por el transcurso de la enfermedad. La persona ya no es la misma.

Los planes de intervención social han de incorporar las funcianes cerebrales que altera cada enfermedad neurabigica, como es el caso de la Ela, donde la sensibilidad e inteligen cia están inalteradas, se debe escuchar los deseos que puede expresar la persona. En el al. zheimer, en cambio, la memoria y los camblos de estado de ánimo son una constante. Esta fase es fundamental dado que el plan de intervención social a disefíar para cada persona será el establecido según la enfermedad que presenta: ictus, alzhéimer, Ela adaptado a la situación personal familiar y social de la persona.

\section{QUINTA FASE. Planificación multidisciplinar al alta hospitalaria.}

Para que el abordaje y tratamiento de la persona sean lo más adecuados posibles, se requiere un trabajo multidisciplinar donde los distintos profesionales puedan planificar y evaluar de forma conjunta la situación de la persona y establecer asl, criterios comunes para dar respuesta a los posibles déficits sufridos por la persona en todas sus áreass fisica, psiquica y social.

Estín establecidas reuniones formales semanales del equipo multidisciplinar de neurologia, dónde la trabajadora social sanitaria de neurologia es la coordinadora de estas: con: voca a los miembros permanentes integrantes, a aquellos que por la necesidad del caso a tratar sea necesaria su presencla y levanta acta de las mismas, cuando la urgencia del caso lo precisa se realizardin reuniones informales. Este plano de trabajo en equipo permite establecer una visión global de las circunstancias y necesidades, donde el objetivo es identificar y posiblitar el recurso mís idóneo al alta, garantizando su recuperación funcional y evitar o postergar reingresos hospitalarios.

A alta hospitalaria se entregard un informe del profesional del trabajo social sanitario a la persona para dar continuidad de la atención sodial.

\section{SEXTA FASE. Seguimiento post-alta hospitalaria.}

El profesional de trabajo social sanitario tiene establecido entomo a los 3,6 meses, un contacto con la persona y famlilares dónde se abordan aspectos de su proceso salud-enfer. medad, que estén influyendo en aquellos, tratamiento, estado de ánimo, organización fa: miliar, limitaciones funcionales, entre otros. El seguimiento está estableció con el objetivo de comprobar el estado de salud de la persona y la evolución de su enfermedad, su grado de satisfacción y la adecuación de los apoyos que está recibiendo, y comprobar la pertinencia de modificar el plan de intervención social inicial. 


\section{CONCLUSIONES Y RECOMENDACIONES}

Recapitulando lo recogido en el articulo, podemos observar que el papel del trabajador social con personas que sufren una enfermedad neurológica es imprescindible, es una realidad la presencia cada vez mayor en nuestra sodedad de personas que las sufren.

La aparición de cualquiera de estas enfermedades en la vida de la persona y su famllia no deja indiferente; lo más grave, la transformación psico-socio-sanitaria y fundonal que sucede en la misma. La persona con la que se inicia una intervención social, en muchas ocasiones, ve dafiada su comunicación verbal, afectada su capacidad cognitiva, la movildad fisica se pierde o reduce, sufre al teraciones del comportamiento, dificultad para tragar alimentos, entre otros. Es indudable, que estos dafios deben incorporarse a la intervención del trabajo social con estos pacientes.

Este articulo, a través de la descripción de las 4 "Q": el "que "definición y funciones, "quien"n" "para que" y "porque" del trabajo soclal en el ímbito de la neurologia, justifica el reconocimiento futuro de una especialidad del trabajo social en este campo.

En palabras de Ituarte (2017) la formación generalista y pregraduada es solo un inicio de preparación para la práctica del trabajo social. La ausencia de especialización en los programas de estudio de trabajo soclal supone la búsqueda de un aprendizaje de posgrado habilitante, que aún hoy en nuestro pais no está disponible en el medio universitario. La persona aspirante a una especializadión del trabajo soclal debe obtenerla por otros medios, no siempre reconocidos $u$ homologados.

Este vacio nos lleva a platear una serie de propuestas, algunas de ellas implementadas en el Hospital Cinico Universitario de Valladolid:

Reconocimiento del perfil especifico de este profesional, que garantice una atención social especializada y de calidad en el campo de la intervención social en neurologla. Es necesario, establecer unos criterios mínimos de capacitación para intervenir en este campo, homogenelzar conocimientos, habilidades, actitudes, competencias, participación en grupos de investigación y experiencia que ha de poseer el trabajo social, promoviendo una integración y formación continuada como miembro permanente para su inclusión en el campo de la neurologia.

Inclusión de la asignatura de neurologia en la formación universitaria de trabajo soclal. El trabajo social precisa una integración de conocimientos clínicos, sociales y psicológicos que le doten de una visión biopsicosoclal. Le facilita el conocimiento de la enfermedad para el disefio de una intervención social individualizada y eficaz, incorpora la influencla de la enfermedad en la persona y familia.

La asignatura de neurologia en la carrera de trabajo social aportaría utilidad también a otros ámbitos de intervención del trabajo social, para comprender procesos de comunicación y recuperación psicosocial eficaz en la persona.

Elaborar instrumentos, protocolos y procedimientos de intervención social, que mues. tren unas acciones ordenadas en el marco de actuación con estas personas. Protocolos y procedimientos adecuados para un desempeño profesional especializado que promuevan una atencidon eficaz y de calidad, agilizando el proceso de intervención y ayudando a es.

[22] Dexumentwi de Trabajo Social / n'63 - 155N 1133-6552 / 15SN Electrónice 2173-82.46 
tablecer rutas de actuadón sólidas y adaptadas a la magritud del dafo provocado en la persona.

Modelo de intervención centrado en, con y para la persona. Las enfermedades neurologicas prowocan trastornos de la comunicación, entendimiento, afectación al movimiento, comportamiento y a la coginición. Por ello, es necesario aplicar un modelo centrado: En la persona; conocimiento la persona que ahora es, su contexto y entorno particular; Con la persona, disefiar la intervencidn junto a ela, manejando instrumentos y técnicas que permitan comunicarse, verificar su capacidad de conocimiento y entendimiento, para respetar su capacidad de decisión y autonomía; Para la persona, la finalidad de la intervención es garantizar atención y bienestar en la persona que es tras una enfermedad neurológica, respetando el principio de autodeterminadión.

El desempefio profesional en este campo tan complejo implica un programa de especializadón universitaria en el que la persona complete dicha formación teórica y príctica.

\section{REFERENCIAS BIBLIOGRÁFICAS}

Arrizabalaga, A (2018), Las enfermedades neurológicas causan el 19 por ciento de muer. tes en Espafia. Recuperada el 3 marzo del 2020 de https://Www.efesalud.com/enfer. medades neurologicas muertes espanal

Arredondo, R., Cosano, F., Gutiérrez, A. M., Morales, M., Ortiz, L, B Rivas, M. (2013) Diccionario Práctico de Trabajo Social, Malaga, Colegio Oficlal de trabajo Social de Malaga.

Askunze, C.; Elzagulre, M. y Urrutia, C. (2004) La sistematización, una nueva mirada a nuestras profticas. Bllbao: Alboan/Hegoa/Instituto de Derechos Humanos/ Pedro Arrupe.

Bamechea, M.: M. y Morgan, M.: L (2010). La sistematización de experienclas: producción de conocimientos desde y para la príctica. Tendencias \& Retos, 15, pp. 97-107. Recuperado el is abril 2020 de httpa//www.ts.ucr.accr/binarios/tendencias/rev-cotendencias 15-07.pdf

Berenguer, L. \& Pérez, A. (2016). Factores de riesgo de los accidentes cerebrovasculares durante un bienio. Medisan, 20(s), 621.629. Recuperado 20 marzo del 2020 de http:0 scielo.sid.cu/pdt/san/vions/sanos205.pdf

Bonilla M. 2017. "El treball social en Patenció a persones amb malaltia crónica awançada des de l'atenció primàrla de salut a proposit d'un cas". Revista de Treball Social. Collegi Oficial de Treball Social de Catalunya, num. 210: 81-92. Recuperado o6 marzo del 2020, de http://www.tscat.cat/download//rts/RTS_20_cat/El_ts_en_malaltia_ crac 3rasnica.pdf

Braidor, N (2013). Parte 1. Como es y fundiona el cerebro. En Braidor, N. (Ed.) Cómo funciona tu cerebro para Dummies (925). Barcelona: Banshee

Gastillero, $\mathrm{O}(20 \mathrm{n})$ ) Los 15 trastornos neurológicos más frecuentes: Enfermedades del sistema nervioso y del cerebro que pueden causar un gran malestar. Recuperado el 19 febrero 2020 de https:/psicologiaymente.com/dinica/trastomos neurologicos frecuentes

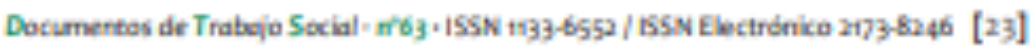


Cambier, J. Masson, M y Dehen, H (2000). Manual de Neurología ( 7 . Ed.). Barcelona: Masson.

Gifuentes, R.M. (2010). Sistemathación de experiencias en trabajo social: desafio inminen te e inaplazable. III Congreso Internacional Trabajo Social Formación profesional in vestigadion sistematización e identidad profesional en la modernidad, preguntas y respuestas. Arequipa PenL. Recuperado el 20 marzo 2020 de http://www.cepalforja. org/sistem/bvirtual/wp-content/Lploads/2016/10/Sisternatizaciac;zBzin-de-exps en. TS desafzCaraDo-Inminente-e-inaplazable-RMCC-dic-ono.pdf

Coloms, D. 2010. "El trabajo soclal sanitario en el marco de la optimización y sostenibel dad del sistema sanitario". Rev. Zerbitzuan. 47: sog-119

Consejo Ceneral de Colegios Oficiales de Farmacéuticos (2016). Prevendión y control de las enfermedades cardiovasculares. Punto Farmacoldglico, 103, 5/25

Del Barrio Alba, A., (2015). Manuales de Neuropsicalogia aplicada: El informe neuropsicoIbgico. Madrid: Editorial Sintesis.

Cracla, A.M. y Forcano, M. (2006). "Estudio de la situación sociofamliar de los pacientes sociosanitarios". Revista Trabajo Social y Salud, 53, 169-180.

Garcla Bustelo, M (2015). Propuesta de creación de una unidad de deterioro cognitivo y demencla en un hospital de distrito. Trabajo Final de Master Universitat Oberta de Ca. talunya, UOC. Recuperado el 13 marzo 2020 de http://openaccess.uoc.edu/webapps/ o2/bitstream/10609/58405/4/mgarcia busteloTFMojnsmemoria.pdf

Cijón, M.T. y Colom, D. (2016). Debates transdisciplinares en tomo a la especialización del trabajo social sanitario. En: D. Carbonero, E. Raya, N. Caparrós y C Cimeno, Res puestas transdisciplinares en una sociedad glabal: Aportaciones desde el Trabajo Social (1.23). Logrono: Universidad de La Rioja. Recuperado o4 abeli del 2020 de https:[/ publicaciones.unirioja_es/Catalogo/online/CIFETS_2016/Monogirafia/pdf/TC 376 .pdf

Ituarte, A. (2001). "Trabajo social en el contexto sanitarioc socioepidemiologla y clinica". Texto del curso impartido en el Título de Especlalista Universitario Salud Pública y Trabajo Social. Departamento de Medicina Preventiva y Salud Pública. Facultad de Farmacia. UPV.EHU. No publicado. En Agencia Valenciana de Salut (2012). Gula de Intervención de Trabajo Sodial Sanitario. Valenclas Generalitat Valenciana.

Ituarte, A. (2017). Actualidad y pertinencla del Trabajo Social Clinica. En A. Ituarte (Coord.), Prácticas del Trabajo Sodal Clínico. (pp. 19-43). Valenda: Nau llibres.

Izquierdo y Ruiz. P. (2568). Importancia del estudio de la etiología en el diagnóstico de las enfermedades: discurso leldo ante el claustro de la universidad central. Universi. dad complutense de Madridz Imprenta de la compahila de impresores y libreros.

Martínez, R. y Miangolarra, J (2006). Capitula 2: Aspectos médicos de la enfermedad del Alzheimer y otras demenclas: Algunos problemas clínicos a evitar en el paciente con demencla. El culdador y la enfermedad de Alzheimer: Formación y asistencla. Editorial Universitarla Ramón Areces, Madrid.45,70.

[24] Doaummtas de Trabojo Social - m'63 + $155 N$ 19336552 / IS5N Electrónico 2173-8246 
Navarro, E, Conde, M.A y Vilanueva, JA (2015). Enfermedad de Alzheimer. [versión electrónica] Revista Mediclne-Programa de Formación Médica Continuada Acreditado, $11(72)$, 4306-4315. Recuperado el 23 abril 2020 de https://www.sciencedirect.com/ science/article/pil/S0304541215000037

Olmedo, V. y Arranz, M. (2017). Trabajo Social Sanitarla: Intervención con Pacientes con ictus desde el Servicio Medico de Neurologia del Hospital Clinico Universitario de Va lladolid. Trabajo Social y Salud (88), pp. 39-47.

Olmedo, V. y Conzallez, R. (2019). "Repasando la intervención psicosodal en pacientes con ictus". Acciones e Investigaciones Sociales.1 (39) \$ 9 -110. https://doi.org/10.26754/ ojs_ais/ais.2019393233

Organización Médica Colegial Espafia (2018). Hoy, Dia Mundial de la Esclerosis Multiple: Se duplica el número de pacientes con esclerosis múltiple en Espańa en las Lítimas dos décadas. Recuperado el 20 abell de http://wwwmedicosypacientes.com/articulo/seduplica-el-numero-de-pacientes-con-esclerosis multiple en-espana-en-las ultimas-dos

Organización Mundial de la Salud (2016). zQué son los trastornos neurolígicos? Recupe rado 10 abril del 2020 de https//www.who.int/features/qa/s5/es/

Pdrez, A (2017, octubre 26). Los casos de ictus se podrían evitar con una adecuada pre. vencidon de las factores de riesgo y un estilo de vida saludable. Departamento de Pren sa de la Socledad Espahola de Neurología. Recuperado 20 marzo 2020, de httpa/fwww. sen.es/saladeprensa/pdf/Link257.pdf

Rillet, F., y Barthez, E ( 1566 ). Tratado cinico y próctico de las enfermedades de los nih́os. Vol. 1. Madrid. Carlos Bally-Balliere.Rodriguez, L.M y Garcia, M.* P (2019). Salud y Tra bajo Soclal: un andilisis desde las perspectivas de la formación y la investigación, en Cuadernos de Traba/o Sodal. 32(1), 123139. httpelldx.doi.org/10.5209/CUTS.57884

SACYL (2016). IV Plan de Salud de Costilla y Ledrs Perspectiva 2aza. Recuperado el 23 mar 202020 de https://Www.saludcastillayleon.es/istitucion/es/planes-estrategias/v-plan salud castilla leon.

Salud a Diario (25 octubre 2017). El impacto socloecondomico de la em en Europa't El 41: de las mujeres dlagnosticadas de esclerosis máltiple se separó de su pareja y el $38 z$ tuvo que dejar su empleo. Recuperado el 29 marzo 2020 de https://www.saludadiario.es/ pacientes-de-cercalel-41-de-as mujeres-diagnosticadas de esclerosis multiple-se-separo-de-suparejay el-38-turo-que-dejar-su-empleo

Sociedad Espafiola de Neurologla (2017). SEN. La Fundación del Cerebro. Recuperado el 15 marzo 2020 de http://www.fundaciandelcerebro.es/index.php/la fundacion

SEN (2018). Impacto de las enfermedades neurologicas en la mortalidad espafiola: LXX Re unión Anual de la SEN. Recuperado el 20 marzo 2020 de httpe//www.sen.es/salade. prensa/pdf/Linkas7informe.pdf 


\section{ANEXOS}

ANEXO 1. PREVALENCIA dE LAS ENFERMEDAdES NEUROLÓGICAS EN ESPAN̂A.

Tabla 1. Incidencla de las enfermedades neurológicas a nivel nacional.

\begin{tabular}{|c|c|}
\hline ENFERMEDAD & INCIDENCIA \\
\hline Alaheimer. & $\begin{array}{l}\text { Caus más trecuente de demencia en mayores de } 65 \text { afios. En Espa/ a } \\
\text { se calcula que pueden existir unas } 500.000 \text { personas con la enferme } \\
\text { dad. }\end{array}$ \\
\hline Epilepsia. & $\begin{array}{l}\text { En Espa/ta una de cada } 200 \text { personas padece epilepsla, (zprox. } 200.000 \\
\text { espaholes) y cada aho } 20.000 \text { personas desarrollan la enfermedad por } \\
\text { primera vez }\end{array}$ \\
\hline Cefaleas o migraflas & 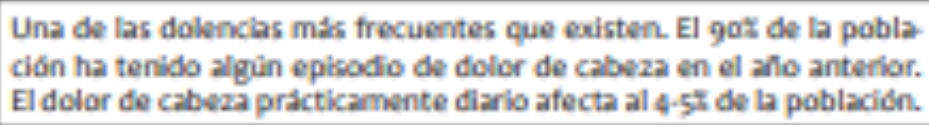 \\
\hline Ictus & $\begin{array}{l}\text { Primera causa de mortalidad entre las mujeres espanolas y la segunda } \\
\text { en las varones. En Espa/ta mueren } 40.000 \text { anualmente por esta causa } \\
\text { Cada seis minutos se produce un ictus en Espana. }\end{array}$ \\
\hline La Esclerosis mûltiple & $\begin{array}{l}\text { Caus más trecuente de incapacidad por entermedad de las personas } \\
\text { jovenes. En Espana hay mas de } 35.000 \text { afectados }\end{array}$ \\
\hline Parkinson & $\begin{array}{l}\text { Segunda enfermedad degenerativa del Sistema Nervioso mas fre } \\
\text { cuente. En España existen más de 80.000 pacientes parkínsonianox. }\end{array}$ \\
\hline $\begin{array}{l}\text { La Enfermedad Corea } \\
\text { de Huntington }\end{array}$ & $\begin{array}{l}\text { En Espa/a, se estima que mas de } 4.000 \text { personas tienen la enferme: } \\
\text { dad y más de } 15.000 \text { afrontan el riesgo de haberla heredada. }\end{array}$ \\
\hline
\end{tabular}

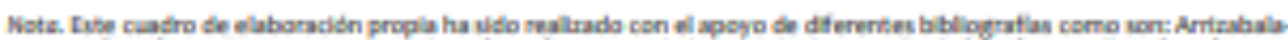

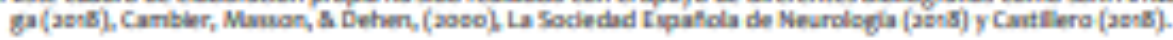

Las enfermedades cerebrovasculares, como el ictus, constituren la primera causa de hospitalización, tanto en hombres como en mujeres, si excluimos en este caso los eplso: dios de embarazo, parto y puerperio, y son las enfermedades que más dias de estancia hos pitalaria causan (IV Plan de Salud de CyL, 2016, p.12\$). A continuación, se presenta el perfil y las funciones del trabajador Social Sanitario en el servicio médico de neurologla.

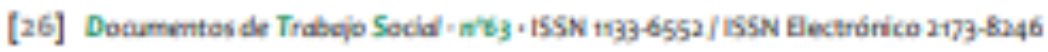


LAS 4"C" DCL TRABAO SOCIAL TN LA ATINCION A PLRSONAS CON

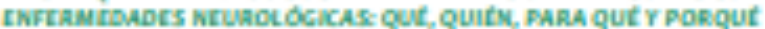

ANEXO 2. TRABANO SOCLAL SANITARIO DE NEUROLOGIA.

Cráfico L. Trabajo Soclal de Neurologla.
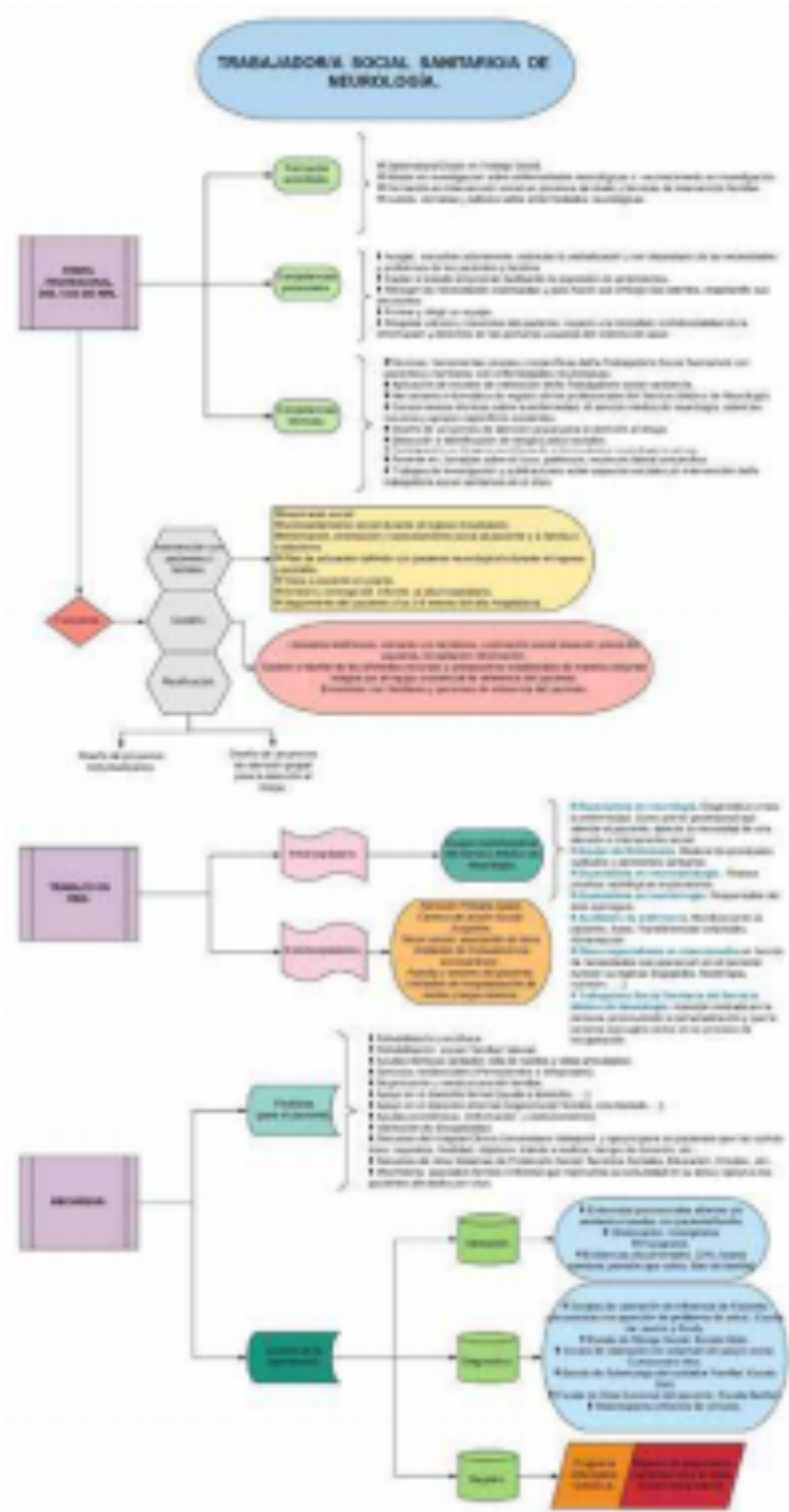

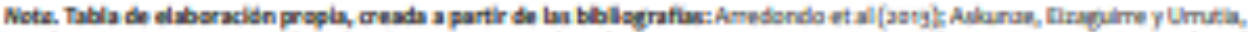

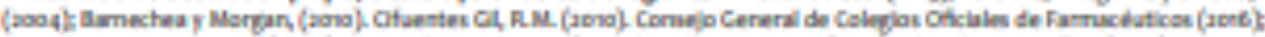

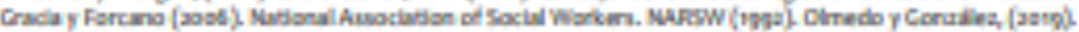

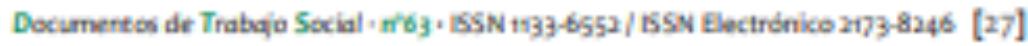


ANEXO 3. FLUJOGRAMA ACTUACIÓN DEL TRABAJO SOCIAL SANITARIO DE NEUROLOGÍA.

HOSPITAL CLÍNICO UNIVERSITARIO VALLADOLID

TRABAJADORIA SOCIAL SANITARIOIA DE NEUROLOGIA.

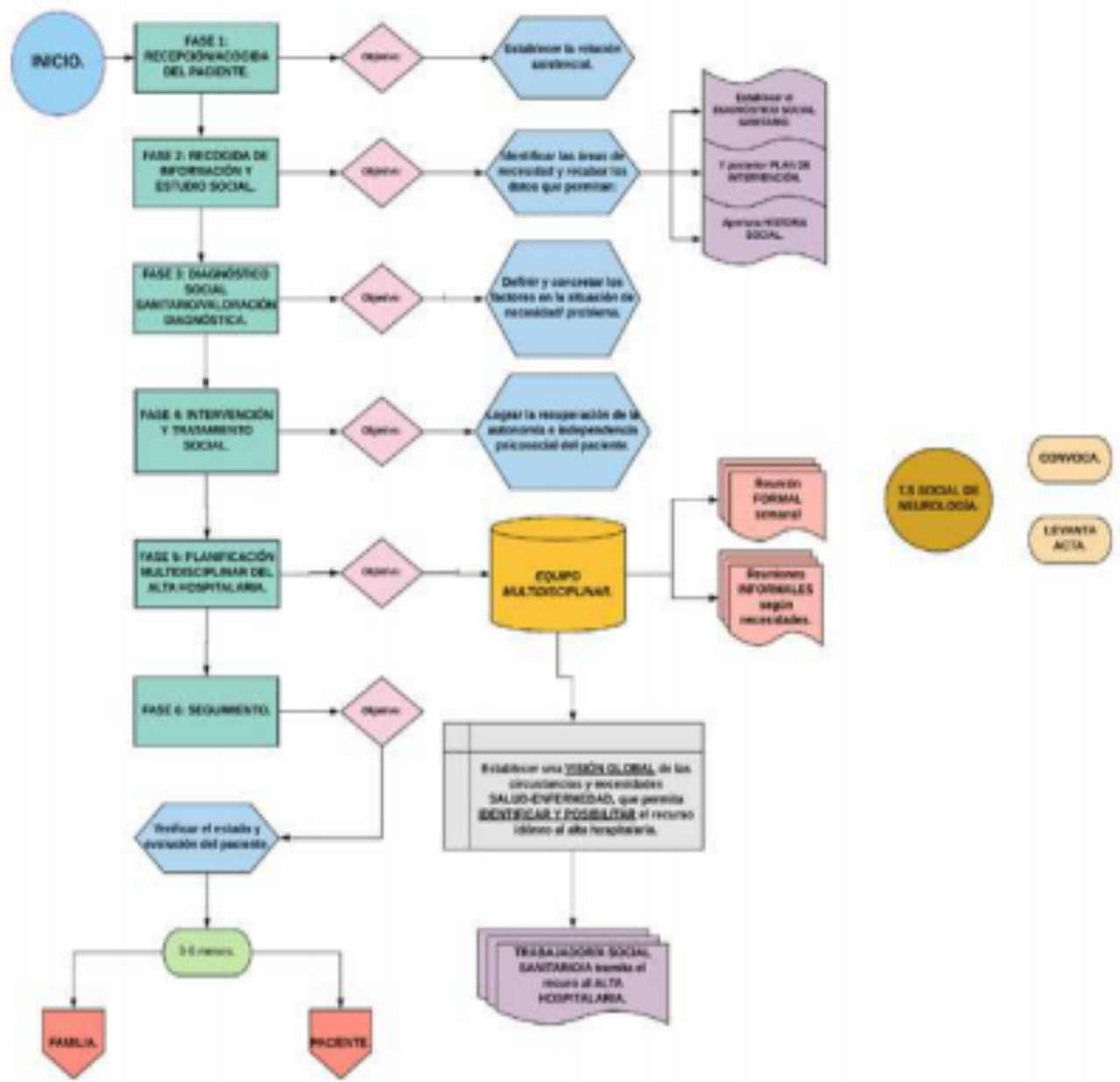

Nota. Elaboración Propia. Flujograma de la actuación de b Trabajadora Social del Hospital Clínico Universitario de Valladolid del área de Neurologia. 


\section{ANEXO 2: ESCALAS DE REGISTRO}

ESCALA MODIFICADA RANKIN

\begin{tabular}{|c|c|c|}
\hline $\mathbf{0}$ & Sin Síntomas & \\
\hline 1 & $\begin{array}{l}\text { Sin } \\
\text { importante }\end{array}$ & $\begin{array}{l}\text { Capaz de realizar sus actividades y obligaciones } \\
\text { habituales }\end{array}$ \\
\hline 2 & Incapacidad leve & $\begin{array}{l}\text { Incapaz de realizar algunas de sus actividades } \\
\text { previas, pero capaz de velar por sus intereses y } \\
\text { asuntos propios }\end{array}$ \\
\hline 3 & Incapacidad moderada & $\begin{array}{l}\text { Síntomas que restringen significativamente su } \\
\text { estilo de vida o impiden su subsistencia totalmente } \\
\text { autónoma (p. eje. Necesitando alguna ayuda) }\end{array}$ \\
\hline 4 & $\begin{array}{l}\text { Incapacidad } \\
\text { moderadamente severa }\end{array}$ & $\begin{array}{l}\text { Síntomas que impiden claramente su subsistencia } \\
\text { independiente aunque sin necesidad de atención } \\
\text { continua (p. ej. Incapacidad para atender sus } \\
\text { necesidades personales sin asistencia) }\end{array}$ \\
\hline 5 & Incapacidad severa & $\begin{array}{l}\text { Totalmente dependiente, necesitando asistencia } \\
\text { constante día y noche. }\end{array}$ \\
\hline 6 & Muerte & \\
\hline
\end{tabular}

Van Swieten JC, Koudstaal PJ, Visser MC, Schouten HJ, van Gijn J. Interobserver agreement for the assessment of handicap in stroke patients. Stroke. 1988;19:604-7.

Bonita R, Beaglehole R. Modification of Rankin Scale: Recovery of motor function after stroke. Stroke. 1988; 19:1497-500 
ESCALA NIHSS

Escala NIHSS: National institute of Health Stroke Scale. Fechas/hora

\begin{tabular}{|c|c|c|c|c|c|c|c|c|c|c|}
\hline 1a. Nivel de conciencia & $\begin{array}{l}\text { Alerta } \\
\text { Somnolencia } \\
\text { Obnubilación } \\
\text { Coma } \\
\end{array}$ & $\begin{array}{l}0 \\
1 \\
2 \\
3 \\
\end{array}$ & $\begin{array}{l}0 \\
1 \\
2 \\
3 \\
\end{array}$ & $\begin{array}{l}0 \\
1 \\
2 \\
3 \\
\end{array}$ & $\begin{array}{l}0 \\
1 \\
2 \\
3 \\
\end{array}$ & $\begin{array}{l}0 \\
1 \\
2 \\
3 \\
\end{array}$ & $\begin{array}{l}0 \\
1 \\
2 \\
3 \\
\end{array}$ & $\begin{array}{l}0 \\
1 \\
2 \\
3 \\
\end{array}$ & $\begin{array}{l}0 \\
1 \\
2 \\
3\end{array}$ & $\begin{array}{l}0 \\
1 \\
2 \\
3\end{array}$ \\
\hline $\begin{array}{l}\text { 1b. Nivel de conciencia Preguntas } \\
\text { verbales } \\
\text { ¿En qué mes vivimos? ¿Qué edad } \\
\text { tiene? }\end{array}$ & $\begin{array}{l}\text { Ambas respuestas son correctas } \\
\text { Una respuesta correcta } \\
\text { Ninguna respuesta correcta }\end{array}$ & $\begin{array}{l}0 \\
1 \\
2\end{array}$ & $\begin{array}{l}0 \\
1 \\
2\end{array}$ & $\begin{array}{l}0 \\
1 \\
2\end{array}$ & $\begin{array}{l}0 \\
1 \\
2\end{array}$ & $\begin{array}{l}0 \\
1 \\
2\end{array}$ & $\begin{array}{l}0 \\
1 \\
2\end{array}$ & $\begin{array}{l}0 \\
1 \\
2\end{array}$ & $\begin{array}{l}0 \\
1 \\
2\end{array}$ & $\begin{array}{l}0 \\
1 \\
2\end{array}$ \\
\hline $\begin{array}{l}\text { 1c. Nivel de conciencia. Órdenes } \\
\text { motoras } \\
\text { 1.Cierre los ojos, después ábralos. } \\
\text { 2.Cierre la mano, después ábrala. }\end{array}$ & $\begin{array}{l}\text { Ambas respuestas son correctas } \\
\text { Una respuesta correcta } \\
\text { Ninguna respuesta correcta }\end{array}$ & $\begin{array}{l}0 \\
1 \\
2\end{array}$ & $\begin{array}{l}0 \\
1 \\
2\end{array}$ & $\begin{array}{l}0 \\
1 \\
2\end{array}$ & $\begin{array}{l}0 \\
1 \\
2\end{array}$ & $\begin{array}{l}0 \\
1 \\
2\end{array}$ & $\begin{array}{l}0 \\
1 \\
2\end{array}$ & $\begin{array}{l}0 \\
1 \\
2\end{array}$ & $\begin{array}{l}0 \\
1 \\
2\end{array}$ & $\begin{array}{l}0 \\
1 \\
2\end{array}$ \\
\hline $\begin{array}{l}\text { 2. Mirada conjugada } \\
\text { (voluntariamente o reflejos } \\
\text { óculocefálicos, no permitidos } \\
\text { óculovestibulares) } \\
\text { Si lesión de un nervio periférico: } \\
\text { 1 punto. }\end{array}$ & $\begin{array}{l}\text { Normal } \\
\text { Paresia parcial de la mirada } \\
\text { Paresia total o desviación forzada }\end{array}$ & $\begin{array}{l}0 \\
1 \\
2\end{array}$ & $\begin{array}{l}0 \\
1 \\
2\end{array}$ & $\begin{array}{l}0 \\
1 \\
2\end{array}$ & $\begin{array}{l}0 \\
1 \\
2\end{array}$ & $\begin{array}{l}0 \\
1 \\
2\end{array}$ & $\begin{array}{l}0 \\
1 \\
2\end{array}$ & $\begin{array}{l}0 \\
1 \\
2\end{array}$ & $\begin{array}{l}0 \\
1 \\
2\end{array}$ & $\begin{array}{l}0 \\
1 \\
2\end{array}$ \\
\hline $\begin{array}{l}\text { 3. Campos visuales (confrontación) } \\
\text { Si ceguera bilateral de cualquier } \\
\text { causa: } 3 \text { puntos. } \\
\text { Si extinción visual: } 1 \text { puntos }\end{array}$ & $\begin{array}{l}\text { Normal } \\
\text { Hemianopsia parcial } \\
\text { Hemianopsia completa } \\
\text { Ceguera bilateral }\end{array}$ & $\begin{array}{l}0 \\
1 \\
2 \\
3 \\
\end{array}$ & $\begin{array}{l}0 \\
1 \\
2 \\
3 \\
\end{array}$ & $\begin{array}{l}0 \\
1 \\
2 \\
3 \\
\end{array}$ & $\begin{array}{l}0 \\
1 \\
2 \\
3 \\
\end{array}$ & $\begin{array}{l}0 \\
1 \\
2 \\
3 \\
\end{array}$ & $\begin{array}{l}0 \\
1 \\
2 \\
3 \\
\end{array}$ & $\begin{array}{l}0 \\
1 \\
2 \\
3 \\
\end{array}$ & $\begin{array}{l}0 \\
1 \\
2 \\
3 .\end{array}$ & $\begin{array}{l}0 \\
1 \\
2 \\
3 \\
\end{array}$ \\
\hline 4. Paresia facial & $\begin{array}{l}\text { Normal. } \\
\text { Paresia leve (asimetría al sonreír.) } \\
\text { Parálisis total de músc. facial inferior } \\
\text { Parálisis total de músc facial superior } \\
\text { e inferior. }\end{array}$ & $\begin{array}{l}0 \\
1 \\
2 \\
3\end{array}$ & $\begin{array}{l}0 \\
1 \\
2 \\
3\end{array}$ & $\begin{array}{l}0 \\
1 \\
2 \\
3\end{array}$ & $\begin{array}{l}0 \\
1 \\
2 \\
3\end{array}$ & $\begin{array}{l}0 \\
1 \\
2 \\
3\end{array}$ & $\begin{array}{l}0 \\
1 \\
2 \\
3\end{array}$ & $\begin{array}{l}0 \\
1 \\
2 \\
3\end{array}$ & $\begin{array}{l}0 \\
1 \\
2 \\
3\end{array}$ & $\begin{array}{l}0 \\
1 \\
2 \\
3\end{array}$ \\
\hline $\begin{array}{l}\text { 5. Paresia de extremidades } \\
\text { superiores (ES) } \\
\text { Se explora } 1^{\circ} \text { la ES no parética } \\
\text { Debe levantar el brazo extendido a } \\
45^{\circ} \text { (decúbito) } \\
\text { ó a } 90^{\circ} \text { (sentado). No se evalúa la } \\
\text { fuerza distal } \\
\text { Se puntúa cada lado por separado. } \\
\text { El } 9 \text { no se contabiliza en el cómputo } \\
\text { global. }\end{array}$ & $\begin{array}{l}\text { Mantiene la posición } 10^{\prime \prime} \text {. } \\
\text { Claudica en menos de } 10^{-1} \text { sin llegar a } \\
\text { tocar la cama. } \\
\text { Claudica y toca la cama en menos de } \\
10^{-} \text {. } \\
\text { Hay movimiento pero no vence } \\
\text { gravedad. } \\
\text { Parálisis completa.. } \\
\text { Extremidad amputada o inmovilizada }\end{array}$ & $\begin{array}{l}0 \\
1 \\
2 \\
3 \\
4 \\
9\end{array}$ & $\begin{array}{l}0 \\
1 \\
2 \\
3 \\
4 \\
9\end{array}$ & $\begin{array}{l}0 \\
1 \\
2 \\
3 \\
4 \\
9\end{array}$ & $\begin{array}{l}0 \\
1 \\
2 \\
3 \\
4 \\
9\end{array}$ & $\begin{array}{l}0 \\
1 \\
2 \\
3 \\
4 \\
9\end{array}$ & $\begin{array}{l}0 \\
1 \\
2 \\
3 \\
4 \\
9\end{array}$ & $\begin{array}{l}0 \\
1 \\
2 \\
3 \\
4 \\
9\end{array}$ & $\begin{array}{l}0 \\
1 \\
2 \\
3 \\
4 \\
9\end{array}$ & $\begin{array}{l}0 \\
1 \\
2 \\
3 \\
4 \\
9\end{array}$ \\
\hline $\begin{array}{l}\text { 6. Paresia de extremidades } \\
\text { inferiores (EI) } \\
\text { Se explora } 1^{\circ} \text { la EI no patética. } \\
\text { Debe levantar la pierna extendida y } \\
\text { mantener a } 30^{\circ} \text {. } \\
\text { Se puntúa cada lado por separado. } \\
\text { El } 9 \text { no se contabiliza en el cómputo } \\
\text { global. }\end{array}$ & $\begin{array}{l}\text { Mantiene la posición } 5^{\circ} \text {. } \\
\text { Claudica en menos de } 5^{\circ} \text { sin llegar a } \\
\text { tocar la cama. } \\
\text { Claudica y toca la cama en menos de } \\
5^{\circ} \text {. } \\
\text { Hay movimiento pero no vence } \\
\text { gravedad. } \\
\text { Parálisis completa. } \\
\text { Extremidad amputada o inmovilizada. }\end{array}$ & $\begin{array}{l}0 \\
1 \\
2 \\
3 \\
4 \\
9\end{array}$ & $\begin{array}{l}0 \\
1 \\
2 \\
3 \\
4 \\
9\end{array}$ & $\begin{array}{l}0 \\
1 \\
2 \\
3 \\
4 \\
9\end{array}$ & $\begin{array}{l}0 \\
1 \\
2 \\
3 \\
4 \\
9\end{array}$ & $\begin{array}{l}0 \\
1 \\
2 \\
3 \\
4 \\
9\end{array}$ & $\begin{array}{l}0 \\
1 \\
2 \\
3 \\
4 \\
9\end{array}$ & $\begin{array}{l}0 \\
1 \\
2 \\
3 \\
4 \\
9\end{array}$ & $\begin{array}{l}0 \\
1 \\
2 \\
3 \\
4 \\
9\end{array}$ & $\begin{array}{l}0 \\
1 \\
2 \\
3 \\
4 \\
9\end{array}$ \\
\hline $\begin{array}{l}\text { 7. Ataxia de las extremidades. } \\
\text { Dedo-nariz y talón-rodilla. } \\
\text { Si déficit motor que impida medir } \\
\text { dismetría: } 0 \text { pt. }\end{array}$ & $\begin{array}{l}\text { Normal. } \\
\text { Ataxia en una extremidad. } \\
\text { Ataxia en dos extremidades. }\end{array}$ & $\begin{array}{l}0 \\
1 \\
2\end{array}$ & $\begin{array}{l}0 \\
1 \\
2\end{array}$ & $\begin{array}{l}0 \\
1 \\
2\end{array}$ & $\begin{array}{l}0 \\
1 \\
2\end{array}$ & $\begin{array}{l}0 \\
1 \\
2\end{array}$ & $\begin{array}{l}0 \\
1 \\
2\end{array}$ & $\begin{array}{l}0 \\
1 \\
2\end{array}$ & $\begin{array}{l}0 \\
1 \\
2\end{array}$ & $\begin{array}{l}0 \\
1 \\
2\end{array}$ \\
\hline $\begin{array}{l}\text { 8. Sensibilidad. } \\
\text { Si obnubilado evaluar la retirada al } \\
\text { estímulo doloroso. } \\
\text { Si déficit bilateral o coma: } 2 \text { puntos. }\end{array}$ & $\begin{array}{l}\text { Normal } \\
\text { Leve o moderada hipoestesia. } \\
\text { Anestesia. }\end{array}$ & $\begin{array}{l}0 \\
1 \\
2\end{array}$ & $\begin{array}{l}0 \\
1 \\
2\end{array}$ & $\begin{array}{l}0 \\
1 \\
2\end{array}$ & $\begin{array}{l}0 \\
1 \\
2\end{array}$ & $\begin{array}{l}0 \\
1 \\
2\end{array}$ & $\begin{array}{l}0 \\
1 \\
2\end{array}$ & $\begin{array}{l}0 \\
1 \\
2\end{array}$ & $\begin{array}{l}0 \\
1 \\
2\end{array}$ & $\begin{array}{l}0 \\
1 \\
2\end{array}$ \\
\hline $\begin{array}{l}\text { 9. Lenguaje. } \\
\text { Si coma: } 3 \text { puntos. } \\
\text { Si intubación o anartria: explorar por } \\
\text { escritura. }\end{array}$ & $\begin{array}{l}\text { Normal. } \\
\text { Afasia leve o moderada. } \\
\text { Afasia grave, no posible entenderse. } \\
\text { Afasia global o en coma }\end{array}$ & $\begin{array}{l}0 \\
1 \\
2 \\
3\end{array}$ & $\begin{array}{l}0 \\
1 \\
2 \\
3\end{array}$ & $\begin{array}{l}0 \\
1 \\
2 \\
3\end{array}$ & $\begin{array}{l}0 \\
1 \\
2 \\
3\end{array}$ & $\begin{array}{l}0 \\
1 \\
2 \\
3\end{array}$ & $\begin{array}{l}0 \\
1 \\
2 \\
3\end{array}$ & $\begin{array}{l}0 \\
1 \\
2 \\
3\end{array}$ & $\begin{array}{l}0 \\
1 \\
2 \\
3\end{array}$ & $\begin{array}{l}0 \\
1 \\
2 \\
3\end{array}$ \\
\hline $\begin{array}{l}\text { 10. Disartria. } \\
\text { Si af asia: } 3 \text { puntos }\end{array}$ & $\begin{array}{l}\text { Normal. } \\
\text { Leve, se le puede entender. } \\
\text { Grave, ininteligible o anartria. } \\
\text { Intubado. No puntúa. }\end{array}$ & $\begin{array}{l}0 \\
1 \\
2 \\
9\end{array}$ & $\begin{array}{l}0 \\
1 \\
2 \\
9\end{array}$ & $\begin{array}{l}0 \\
1 \\
2 \\
9\end{array}$ & $\begin{array}{l}0 \\
1 \\
2 \\
9\end{array}$ & $\begin{array}{l}0 \\
1 \\
2 \\
9\end{array}$ & $\begin{array}{l}0 \\
1 \\
2 \\
9\end{array}$ & $\begin{array}{l}0 \\
1 \\
2 \\
9\end{array}$ & $\begin{array}{l}0 \\
1 \\
2 \\
9\end{array}$ & $\begin{array}{l}0 \\
1 \\
2 \\
9\end{array}$ \\
\hline $\begin{array}{l}\text { 11. Extinción-Negligencia- } \\
\text { Inatención. } \\
\text { Si coma: } 2 \text { puntos. }\end{array}$ & $\begin{array}{l}\text { Normal. } \\
\text { Inatención/extinción en una modalidad } \\
\text { Inatención/extinción en más de una } \\
\text { modalidad. }\end{array}$ & $\begin{array}{l}0 \\
1 \\
2\end{array}$ & $\begin{array}{l}0 \\
1 \\
2\end{array}$ & $\begin{array}{l}0 \\
1 \\
2\end{array}$ & $\begin{array}{l}0 \\
1 \\
2\end{array}$ & $\begin{array}{l}0 \\
1 \\
2\end{array}$ & $\begin{array}{l}0 \\
1 \\
2\end{array}$ & $\begin{array}{l}0 \\
1 \\
2\end{array}$ & $\begin{array}{l}0 \\
1 \\
2\end{array}$ & $\begin{array}{l}0 \\
1 \\
2\end{array}$ \\
\hline TOTAL & & & & & & & & & & \\
\hline
\end{tabular}

Montañer J, Álvarez-Sabin J. La escala de ictus del National Institute of Health (NIHSS) y su adaptación al español. Neurologia. 2006;21(4):192-202. 


\section{ESCALA GIJÓN}

\section{ESCALA "GIJÓN" DE VALORACIÓN SOCIO-FAMILIAR EN EL ANCIANO}

Indicación: valoración inicial sociofamiliar en el anciano para la detección de riesgo social. Administración: Heteroadministrada.

Interpretación:. Recoge cinco áreas de valoración: situación familiar, situación económica, vivienda, relaciones sociales y apoyo de las redes sociales.

De 5 a 9 Buena/aceptable situación social

De 10 a 14 Existe riesgo social

Más de 15 Problema social

Reevaluación: Cuando las condiciones sociales cambien o se sospeche riesgo social.

Propiedades psicométricas Se ha estudiado la validez de criterio, utilizando como criterio el juicio experto de un profesional experto. No se ha estudiado ni la validez de contenido ni la validez de constructo. La fiabilidad interobservador es muy elevada (indice de kappa de $0,957)$. La consistencia interna es muy baja( alfa de Crombach 0,142 ).

ESCALA "GIJÓN" DE VALORACIÓN SOCIO-FAMILIAR EN EL ANCIANO

\section{A- SITUACION FAMILIAR \\ 1. Vive con familia sin dependencia físico/psiquica. \\ 2. Vive con cónyuge de similar edad. \\ 3. Vive con familia y/o cónyuge y presenta algûn grado de dependencia. \\ 4. Vive solo y tiene hijos próximos. \\ 5. Vive solo y carece de hijos o viven alejados.}

\section{B- SITUACION ECONOMICA1:}

1. Más de 1,5 veces el salario minimo.

2. Desde 1,5 veces el salario mínimo hasta el salario minimo exclusive.

3. Desde el salario mínimo a pensión minima contributiva.

4. L.I.S.M.I. - F.A.S. - Pensión no contributiva.

5. Sin ingresos o inferiores al apartado anterior.

\section{C- VIVIENDA:}

1. Adecuada a necesidades.

2. Barreras arquitectónicas en la vivienda o portal de la casa (peldaños, puertas, estrechas, baños..)

3. Humedades, mala higiene, equipamiento inadecuado (baño incompleto, ausencia de agua caliente, calefacción).

4. Ausencia ascensor, teléfono.

5. Vivienda inadecuada (chabolas, vivienda declarada en ruina, ausencia de equipamientos minimos).

\section{D-RELACIONES SOCIALES:}

1. Relaciones sociales.

2. Relación social sólo con familia y vecinos

3. Relación social sólo con familia o vecinos.

4. No sale del domicilio, recibe visitas.

5. No sale y no recibe visitas.

\section{E-APOYOS DE LA RED SOCIAL:}

1. Con apoyo familiar o vecinal.

2. Voluntariado social, ayuda domiciliaria.

3. No tiene apoyo.

4. Pendiente de ingreso en Residencia Geriátrica.

5. Tiene cuidados permanentes.

Observaciones:

\section{Dx social:}

1.- El cálculo individual de los ingresos, en el caso de matrimonios se obtendrá de la suma de las pensiones de ambos más $1 / 3$ del SMI. dividiendo el resultado entre 2

Gerencia Regional de Salud de Castilla y León. Anexo 33. Escala Gijón: Valoración SocioFamiliar. En Cartera de Servicios de Atención Primaria. [Internet]. Valladolid: Junta de Castilla y León;2019 [citado 21 de marzo de 2021];284. Recuperado a partir de:

https://www.saludcastillayleon.es/institucion/es/catalogo-prestaciones/cartera-servicios/carteraservicios-atencion-primaria.ficheros/1522344-cartera $\% 20 \mathrm{de} \% 20$ servicios $\% 202019$.pdf 


\section{INDICE BARTHEL}

\section{Índice de Barthel}

\begin{tabular}{|c|c|c|}
\hline Item & Situación del paciente & Puntos \\
\hline Comer & $\begin{array}{l}\text { - Totalmente independiente } \\
\text { - Necesita ayuda para cortar carne, el pan, etc. } \\
\text { - Dependiente }\end{array}$ & $\begin{array}{c}10 \\
5 \\
0\end{array}$ \\
\hline Lavarse & $\begin{array}{l}\text { - Independiente: entra y sale solo del baño } \\
\text { - Dependiente }\end{array}$ & $\begin{array}{l}\mathbf{5} \\
\mathbf{0}\end{array}$ \\
\hline Vestirse & $\begin{array}{l}\text { - Independiente: capaz de ponerse y de quitarse la } \\
\text { ropa, abotonarse, atarse los zapatos } \\
\text { - Necesita ayuda } \\
\text { - Dependiente }\end{array}$ & $\begin{array}{c}10 \\
5 \\
0\end{array}$ \\
\hline Arreglarse & $\begin{array}{l}\text { - Independiente para lavarse la cara, las manos, } \\
\text { peinarse, afeitarse, maquillarse, etc. } \\
\text { - Dependiente }\end{array}$ & $\begin{array}{l}\mathbf{5} \\
\mathbf{0}\end{array}$ \\
\hline $\begin{array}{l}\text { Deposiciones } \\
\text { (Valórese la } \\
\text { semana } \\
\text { previa) }\end{array}$ & $\begin{array}{l}\text { - Continencia normal } \\
\text { - Ocasionalmente algún episodio de incontinencia, o } \\
\text { necesita ayuda para administrarse supositorios o } \\
\text { lavativas } \\
\text { - Incontinencia }\end{array}$ & $\begin{array}{l}10 \\
5 \\
0\end{array}$ \\
\hline $\begin{array}{l}\text { Micción } \\
\text { (Valórese la } \\
\text { semana } \\
\text { previa) }\end{array}$ & $\begin{array}{l}\text { - Continencia normal, o es capaz de cuidarse de la } \\
\text { sonda si tiene una puesta } \\
\text { - Un episodio diario como máximo de incontinencia, o } \\
\text { necesita ayuda para cuidar de la sonda } \\
\text { - Incontinencia }\end{array}$ & $\begin{array}{l}10 \\
5 \\
0\end{array}$ \\
\hline $\begin{array}{l}\text { Usar } \quad \text { el } \\
\text { retrete }\end{array}$ & $\begin{array}{l}\text { - Independiente para ir al cuarto de aseo, quitarse y } \\
\text { ponerse la ropa... } \\
\text { - Necesita ayuda para ir al retrete, pero se limpia solo } \\
\text { - Dependiente }\end{array}$ & $\begin{array}{c}10 \\
5 \\
0\end{array}$ \\
\hline Trasladarse & $\begin{array}{l}\text { - Independiente para ir del sillón a la cama } \\
\text { - Minima ayuda física o supervisión para hacerlo } \\
\text { - Necesita gran ayuda, pero es capaz de mantenerse } \\
\text { sentado solo } \\
\text { - Dependiente }\end{array}$ & $\begin{array}{c}15 \\
10 \\
5 \\
0\end{array}$ \\
\hline Deambular & $\begin{array}{l}\text { - Independiente, camina solo } 50 \text { metros } \\
\text { - Necesita ayuda física o supervisión para caminar } 50 \\
\text { metros } \\
\text { - Independiente en silla de ruedas sin ayuda } \\
\text { - Dependiente }\end{array}$ & $\begin{array}{c}15 \\
10 \\
5 \\
1\end{array}$ \\
\hline Escalones & $\begin{array}{l}\text { - Independiente para bajar y subir escaleras } \\
\text { - Necesita ayuda física o supervisión para hacerlo } \\
\text { - Dependiente }\end{array}$ & $\begin{array}{c}10 \\
5 \\
0\end{array}$ \\
\hline \multicolumn{3}{|l|}{ Total: } \\
\hline
\end{tabular}

Máxima puntuación: 100 puntos (90 si va en silla de ruedas)

Índice de Barthel [Internet]. Wikipedia. 2021 [citado el 20 de marzo de 2021]. Recuperado a partir de: https://es.wikipedia.org/wiki/Índice_de_Barthel 


\section{ESCALA APGAR FAMILIAR}

\begin{tabular}{|l|c|c|c|}
\cline { 2 - 4 } \multicolumn{1}{c|}{} & \begin{tabular}{c} 
Casi \\
Nunca: \\
\multicolumn{1}{c|}{}
\end{tabular} & $\begin{array}{c}\text { A veces: } \\
1\end{array}$ & $\begin{array}{c}\text { Casi } \\
\text { Me satisface la ayuda que recibo de mi familia cuando tengo algún } \\
\text { problema y/o necesidad }\end{array}$ \\
\hline $\begin{array}{l}\text { Me satisface la forma como mi familia habla de las cosas y comparte los } \\
\text { problemas conmigo }\end{array}$ & & & \\
\hline $\begin{array}{l}\text { Me satisface como mi familia acepta y apoya mis deseos de emprender } \\
\text { nuevas actividades }\end{array}$ & & & \\
\hline $\begin{array}{l}\text { Me satisface como mi familia expresa afecto y responde a mis } \\
\text { emociones como rabia, tristeza o amor }\end{array}$ & & & \\
\hline $\begin{array}{l}\text { Me satisface cómo compartimos en familia el tiempo de estar juntos, los } \\
\text { espacios en la casa o el dinero }\end{array}$ & & & \\
\hline
\end{tabular}

Suarez M, Alcala M. Apgar familiar: una herramienta para detectar disfunción familiar. Rev. Méd. La Paz [Internet].2014 [citado el 20 de abril de 2021];20. Recuperado a partir de: http://www.scielo.org.bo/pdf/rmcmlp/v20n1/v20n1 a10.pdf 


\section{ANEXO 3: MAPAS}

\section{Mapa 1: ZONAS BÁSICAS DE SALUD URBANAS. AREA SALUD VALLADOLID ESTE}

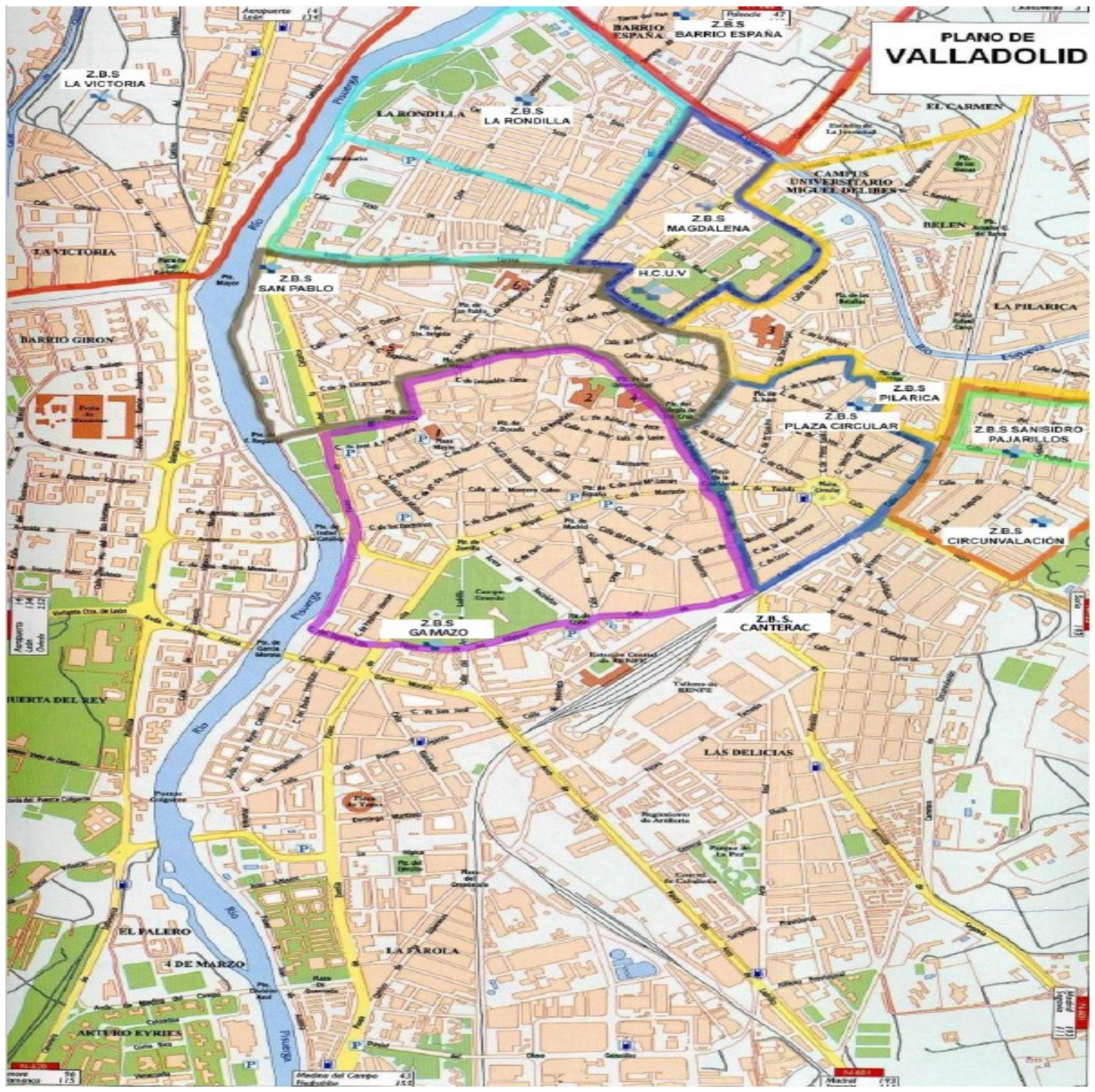


Mapa 2: ZONAS BÁSICAS DE SALUD RURALES. AREA SALUD VALLADOLID ESTE.

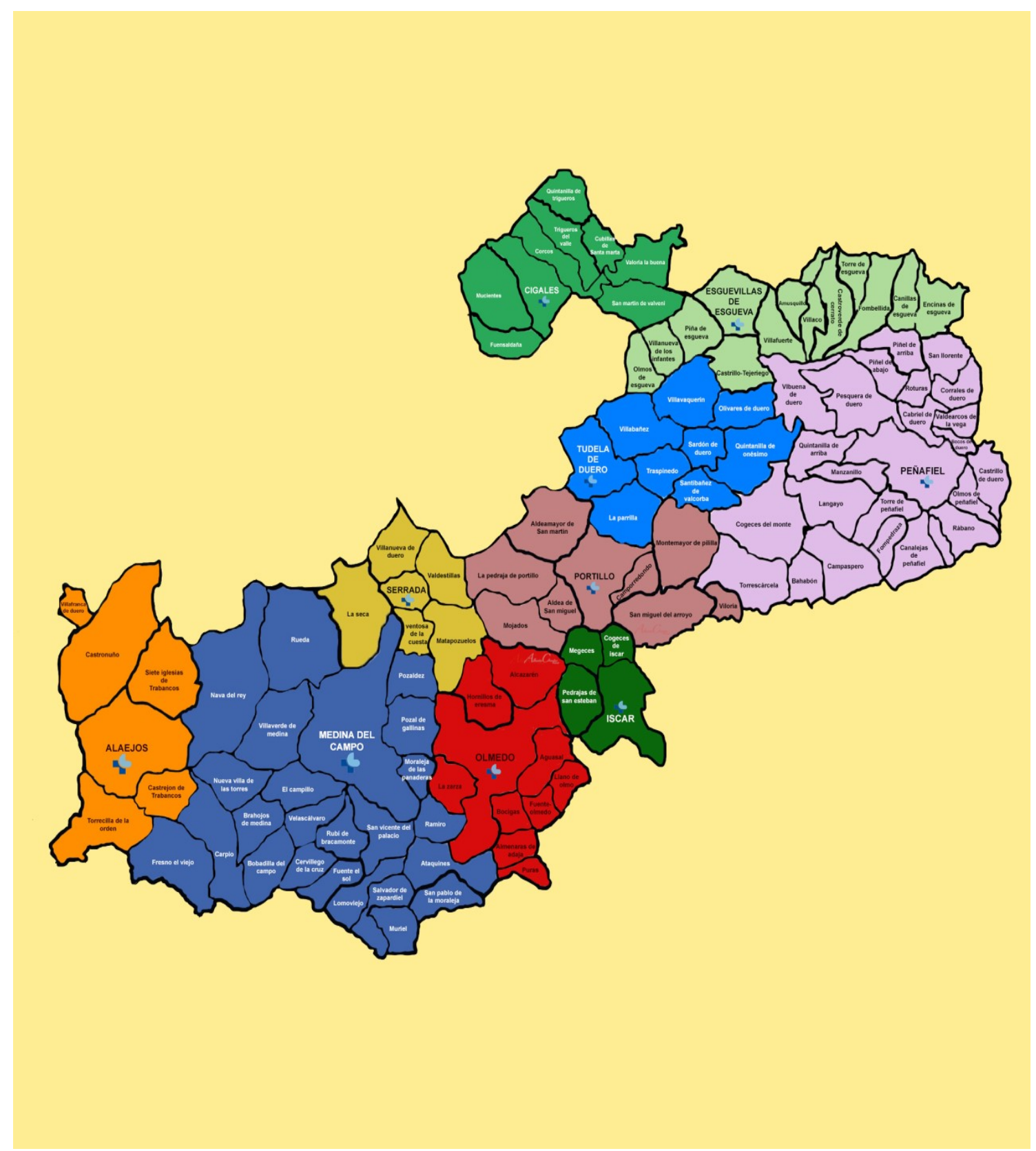

